\title{
Bioinspired Synthesis of Nortriterpenoid Propindilactone G
}

\author{
Yu Wang, ${ }^{\dagger}$ Bo Chen, ${ }^{\dagger}$ Xubiao He and Jinghan Gui* \\ CAS Key Laboratory of Synthetic Chemistry of Natural Substances, Center for Excellence in \\ Molecular Synthesis, Shanghai Institute of Organic Chemistry, University of Chinese Academy of \\ Sciences, \\ Chinese Academy of Sciences, 345 Lingling Road, Shanghai 200032, China \\ †These authors contributed equally to this work. *Correspondence to: guijh@sioc.ac.cn.
}

\section{Supporting Information}

\section{Table of Contents}

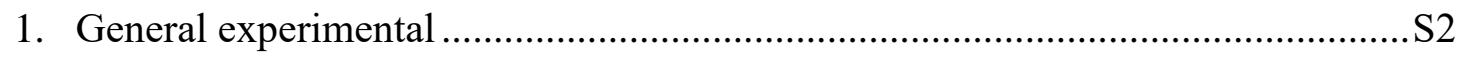

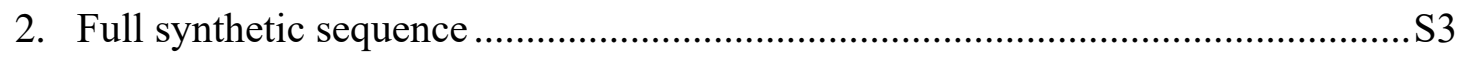

3. Experimental procedures and characterization data for compounds $\mathbf{1 0 - 3 1} \ldots \ldots \ldots . . . \mathrm{S} 5$

4. NMR comparison of synthetic and natural propindilactone $\mathrm{G}$..........................S30

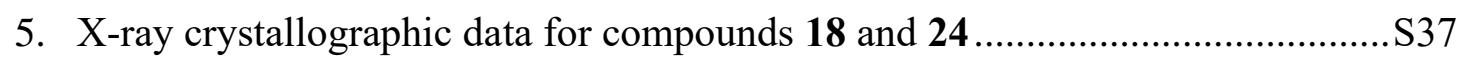

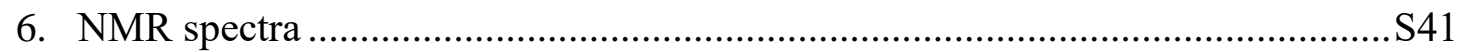

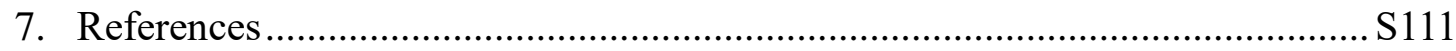




\section{General experimental}

All reactions utilizing air- or moisture-sensitive reagents were carried out in flamedried glassware under an argon atmosphere, unless otherwise stated. Dry tetrahydrofuran (THF), dichloromethane (DCM), toluene (PhMe), diethyl ether $\left(\mathrm{Et}_{2} \mathrm{O}\right)$ were obtained by passing the HPLC grade or pre-dried solvents through activated alumina columns. Tetrachloromethane $\left(\mathrm{CCl}_{4}\right)$, 1,2-dichloroethane (DCE), cyclohexane, ${ }^{t} \mathrm{BuOH}$, triethylamine $\left(\mathrm{Et}_{3} \mathrm{~N}\right)$ and hexamethylphosphoramide (HMPA) were distilled from $\mathrm{CaH}_{2}$. Reagents were purchased at the highest commercial quality and used without further purification, unless otherwise stated. Reactions were magnetically stirred and monitored by thin layer chromatography (TLC) with $0.15-0.2 \mathrm{~mm}$ precoated silica gel $(10-40 \mu \mathrm{m})$ plates, using UV light as the visualizing agent or ethanolic phosphomolybdic acid and heating as developing agents. Flash chromatography was performed with silica gel (200-300 mesh) under pressure. Yields refer to chromatographically and spectroscopically ( ${ }^{1} \mathrm{H}$ NMR) homogeneous material, unless otherwise stated. NMR spectra were recorded on Bruker-400 and Bruker-600 spectrometers. ${ }^{1} \mathrm{H}$ NMR spectra were calibrated using residual undeuterated solvent as an internal reference $\left(\mathrm{CDCl}_{3}: 7.26 \mathrm{ppm} ; \mathrm{MeOH}-d_{4}: 3.31 \mathrm{ppm} ; \mathrm{C}_{5} \mathrm{D}_{5} \mathrm{~N}: 8.74 \mathrm{ppm}\right)$ and ${ }^{13} \mathrm{C}$ NMR spectra were calibrated against the deuterated solvent peak $\left(\mathrm{CDCl}_{3}: 77.2 \mathrm{ppm}\right.$; MeOH- $\left.d_{4}: 49.0 \mathrm{ppm} ; \mathrm{C}_{5} \mathrm{D}_{5} \mathrm{~N}: 150.3 \mathrm{ppm}\right)$. The following abbreviations were used to explain multiplicities: $\mathrm{s}=$ singlet, $\mathrm{d}=$ doublet, $\mathrm{t}=$ triplet, $\mathrm{q}=$ quartet, $\mathrm{m}=$ multiplet, $\mathrm{br}$ $=$ broad. IR spectra were collected on Avatar 330 FT-IR spectrometer. Melting points were determined on SGW X-4 microscopic melting point apparatus and were uncorrected. Optical rotations were determined on JASCO P-1030 Polarimeter in the solvent indicated. High-resolution mass spectra were recorded on IonSpec 4.7 Tesla FTMS or Bruker Daltonics, Inc. APEXIII 7.0 TESLA FTMS. 


\section{Full synthetic sequence}

\section{Scheme S1. Bioinspired Synthesis of Advanced Intermediate Lactone 23}
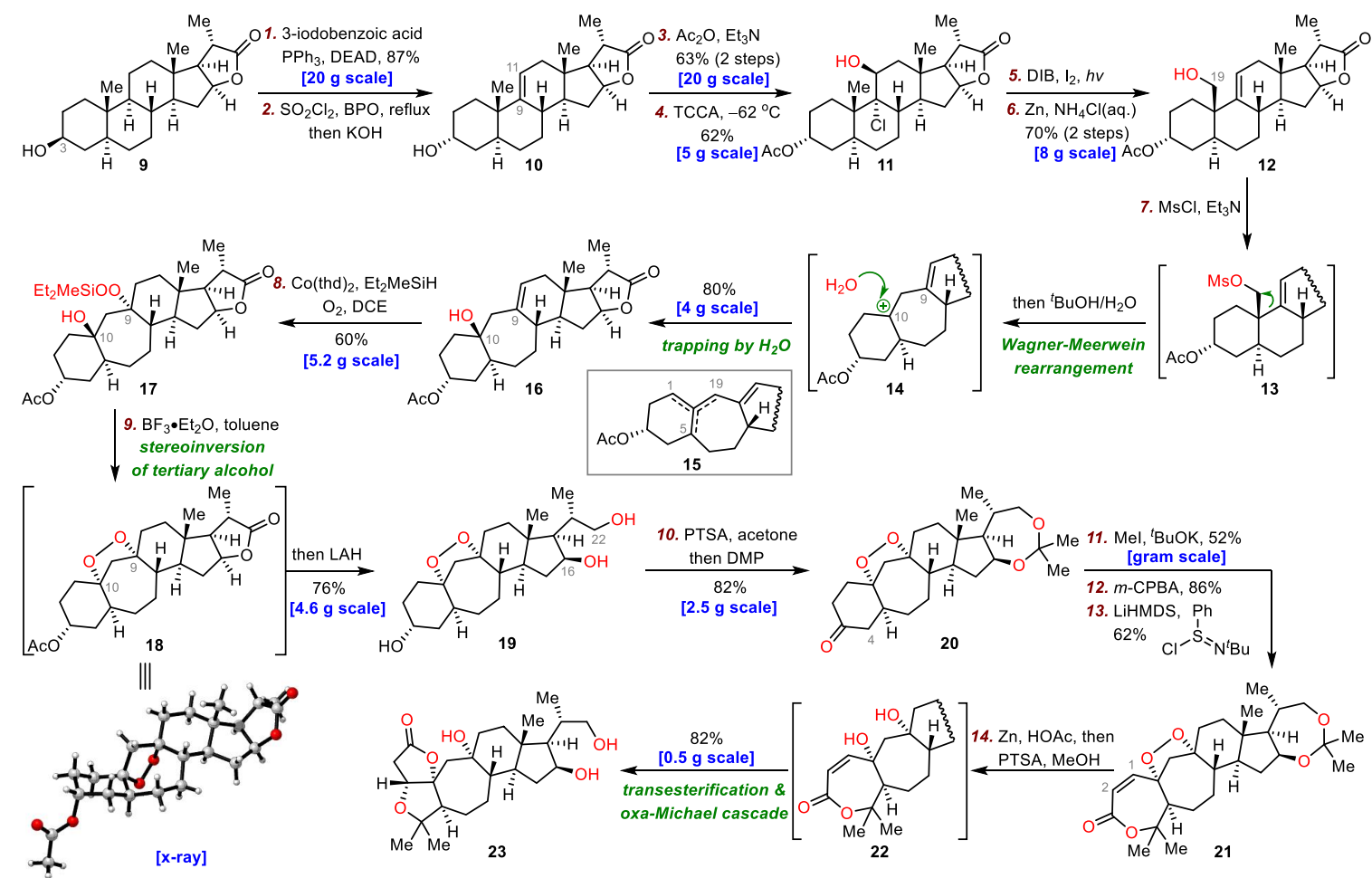

Reagents and conditions: (1) 3-iodobenzoic acid (1.2 equiv), $\mathrm{PPh}_{3}$ (1.5 equiv), DEAD (1.5 equiv), THF, $0{ }^{\circ} \mathrm{C}$ to rt, $2.5 \mathrm{~h}, 87 \%$; (2) $\mathrm{SO}_{2} \mathrm{Cl}_{2}$ (1.6 equiv), $\mathrm{BPO}$ ( 0.2 equiv), $\mathrm{CCl}_{4}$, reflux, $5 \mathrm{~h}$; then $\mathrm{KOH}$ (18.5 equiv), $\mathrm{MeOH}$, reflux, $2 \mathrm{~h}$; then $2.4 \mathrm{M} \mathrm{HCl}$ (aq.), rt, $0.5 \mathrm{~h}$; (3) $\mathrm{Ac}_{2} \mathrm{O}$ (1.3 equiv), $\mathrm{Et}_{3} \mathrm{~N}$ (1.3 equiv), DMAP (0.16 equiv), DCM, rt, 2 h, 63\% (2 steps); (4) TCCA (0.8 equiv), diglyme/ $\mathrm{H}_{2} \mathrm{O}(9 / 1)$, $-62{ }^{\circ} \mathrm{C}, 2.5 \mathrm{~h}, 62 \%$; (5) DIB (3.0 equiv), $\mathrm{I}_{2}$ (3.0 equiv), cyclohexane, $h v(275 \mathrm{~W})$, reflux, $4 \mathrm{~h}$; (6) $\mathrm{Zn}$ (25.0 equiv), $\mathrm{NH}_{4} \mathrm{Cl}$ (10.0 equiv), sat. $\mathrm{NH}_{4} \mathrm{Cl}$ (aq.), $\mathrm{DMF}, 140{ }^{\circ} \mathrm{C}, 4 \mathrm{~h}, 70 \%$ (2 steps); (7) $\mathrm{MsCl}$ (2.0 equiv), DMAP (0.2 equiv), $\mathrm{Et}_{3} \mathrm{~N}$ (3.0 equiv), DCM, rt, $1 \mathrm{~h}$; then ${ }^{t} \mathrm{BuOH} / \mathrm{H}_{2} \mathrm{O}(1 / 2), 20$ to $30{ }^{\circ} \mathrm{C}, 24$ h, $80 \%$; (8) $\mathrm{Et}_{2} \mathrm{MeSiH}$ (1.5 equiv), Co(thd) 2 (0.2 equiv), $\mathrm{O}_{2}$, DCE, rt to $0{ }^{\circ} \mathrm{C}, 9.5 \mathrm{~h}, 60 \%$; (9) $\mathrm{BF}_{3} \cdot \mathrm{Et}_{2} \mathrm{O}$ ( 0.4 equiv), toluene, $50{ }^{\circ} \mathrm{C}, 2 \mathrm{~h}$; then $\mathrm{NaHCO}_{3}$ ( 0.5 equiv); then $\mathrm{LiAlH}_{4}$ (5.0 equiv), THF, $0{ }^{\circ} \mathrm{C}$ to rt, $70 \mathrm{~min}, 76 \%$; (10) 2,2-dimethoxypropane (18.0 equiv), PTSA ( 0.25 equiv), acetone, rt, $0.5 \mathrm{~h}$; then $\mathrm{Et}_{3} \mathrm{~N}$ (2.0 equiv); then DMP (2.5 equiv), $\mathrm{NaHCO}_{3}$ (5.0 equiv), DCM, rt, $1 \mathrm{~h}, 82 \%$; (11)

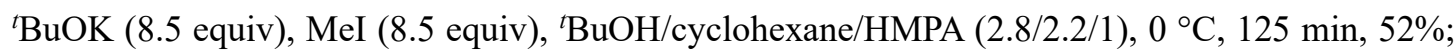
(12) $m$-CPBA (4.0 equiv), $\mathrm{NaHCO}_{3}$ (6.3 equiv), toluene, $\mathrm{rt}, 5 \mathrm{~h}, 86 \%$; (13) LHMDS (1.4 equiv), $\mathrm{N}$ tert-butyl phenylsulfinimidoyl chloride (1.4 equiv), THF, $-78{ }^{\circ} \mathrm{C}$ to $0{ }^{\circ} \mathrm{C}, 80 \mathrm{~min}, 62 \%$; (14) $\mathrm{Zn}(6.0$ equiv), AcOH (6.0 equiv), DCM, rt, $22 \mathrm{~h}$; then PTSA (15.0 equiv), $\mathrm{MeOH}, \mathrm{rt}, 50 \mathrm{~min}, 82 \%$. Abbreviations: $\mathrm{DEAD}=$ diethyl azodicarboxylate; $\mathrm{THF}=$ tetrahydrofuran; $\mathrm{BPO}=$ benzoyl peroxide; DMAP = 4-dimethylaminopyridine; $\mathrm{DCM}=$ dichloromethane; TCCA = trichloroisocyanuric acid; $\mathrm{DIB}=($ diacetoxyiodo $)$ benzene; $\mathrm{DMF}=N, N$-dimethylformamide; thd $=2,2,6,6$-tetramethyl-3,5heptanedionato; $\mathrm{DCE}=1$,2-dichloroethane; $\mathrm{LAH}=$ lithium aluminum hydride; $\mathrm{PTSA}=p$ toluenesulfonic acid; DMP $=$ Dess-Martin periodinane; HMPA $=$ hexamethylphosphoramide; $m$ - 


\section{Scheme S2. Mukaiyama Hydration of Homoallylic Alcohol 16}

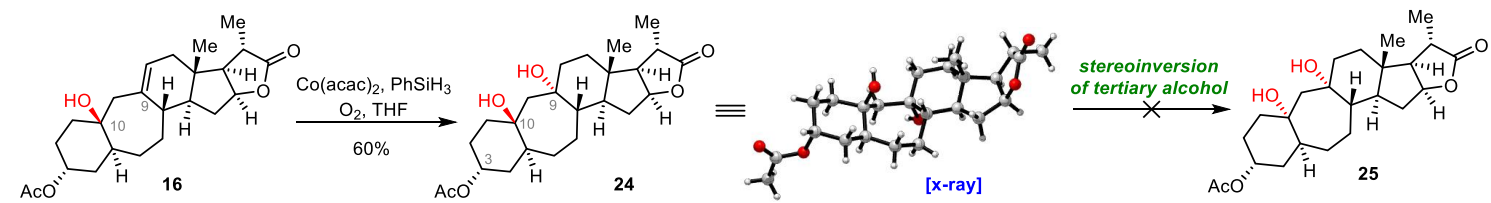

Reagents and conditions: $\mathrm{Co}(\mathrm{acac})_{2}$ ( 0.3 equiv), $\mathrm{PhSiH}_{3}$ (4.0 equiv), $\mathrm{O}_{2}$ ( 1 atm), THF, rt, $5 \mathrm{~h}, 60 \%$. Abbreviation: acac $=$ acetylacetonate.

\section{Scheme S3. Completion of the Synthesis of Propindilactone G (1)}

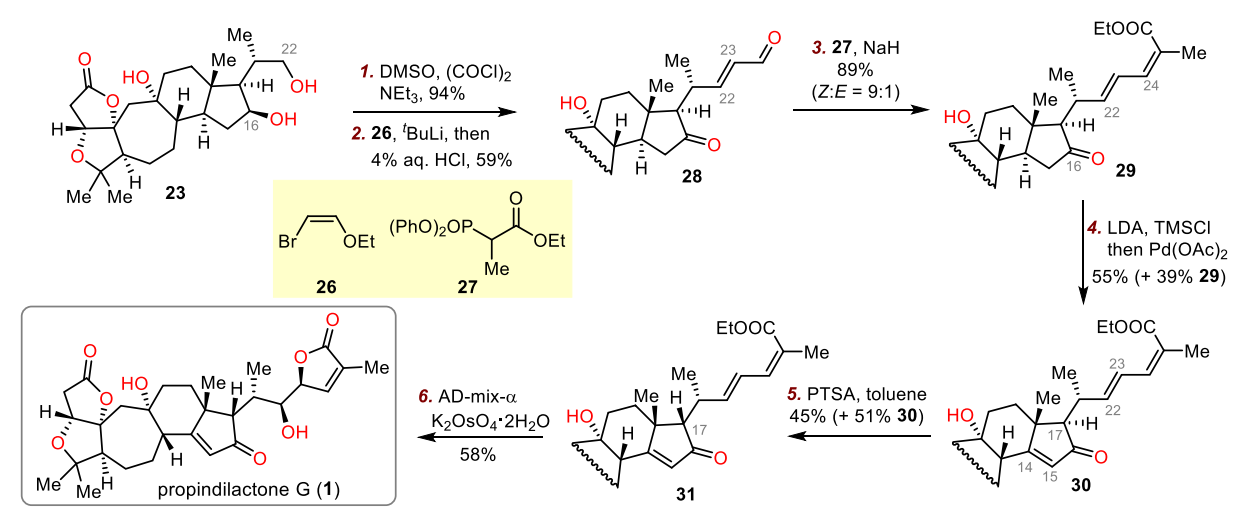

Reagents and conditions: (1) $(\mathrm{COCl})_{2}$ (5.0 equiv), DMSO (7.5 equiv), $\mathrm{Et}_{3} \mathrm{~N}$ (12.0 equiv), DCM, $78{ }^{\circ} \mathrm{C}$ to $0{ }^{\circ} \mathrm{C}, 3 \mathrm{~h}, 94 \%$; (2) 26 (3.5 equiv), ${ }^{t} \mathrm{BuLi}$ (7.0 equiv), $\mathrm{Et}_{2} \mathrm{O} / \mathrm{THF}(3.5 / 1),-78^{\circ} \mathrm{C}, 110 \mathrm{~min}$; then $4 \% \mathrm{HCl}$ (aq.), $\mathrm{H}_{2} \mathrm{O}$, rt, $0.5 \mathrm{~h}, 59 \%$; (3) 27 (2.5 equiv), $\mathrm{NaH}$ (2.8 equiv), THF, $0{ }^{\circ} \mathrm{C}$ to $-78{ }^{\circ} \mathrm{C}$, $2.5 \mathrm{~h}, 89 \%$ ( $Z: E=9: 1)$; (4) LDA (3.0 equiv), TMSCl (4.0 equiv), DME/THF (8/1), $-78^{\circ} \mathrm{C}, 2 \mathrm{~h}$; then $\mathrm{Pd}(\mathrm{OAc})_{2}\left(1.0\right.$ equiv), DMSO, $50{ }^{\circ} \mathrm{C}, 1 \mathrm{~h}, 55 \% 30+39 \%$ 29; (5) PTSA ( 0.2 equiv), toluene, $80{ }^{\circ} \mathrm{C}$, $1 \mathrm{~h}, 45 \% 31+51 \% 30$; (6) AD-mix- $\alpha$ (1.0 equiv), $\mathrm{MeSO}_{2} \mathrm{NH}_{2}$ (1.0 equiv), $\mathrm{K}_{2} \mathrm{OsO}_{4} \cdot 2 \mathrm{H}_{2} \mathrm{O}$ (0.03 equiv), ${ }^{t} \mathrm{BuOH} / \mathrm{H}_{2} \mathrm{O}(1 / 1)$, rt to $0{ }^{\circ} \mathrm{C}, 22.5 \mathrm{~h}, 58 \%$. Abbreviations: $\mathrm{DMSO}=$ dimethylsulfoxide; $\mathrm{LDA}$ $=$ lithium diisopropylamide; $\mathrm{TMS}=$ trimethylsilyl; $\mathrm{DME}=1,2$-dimethoxyethane. 
3. Experimental procedures and characterization data for compounds 10-31.

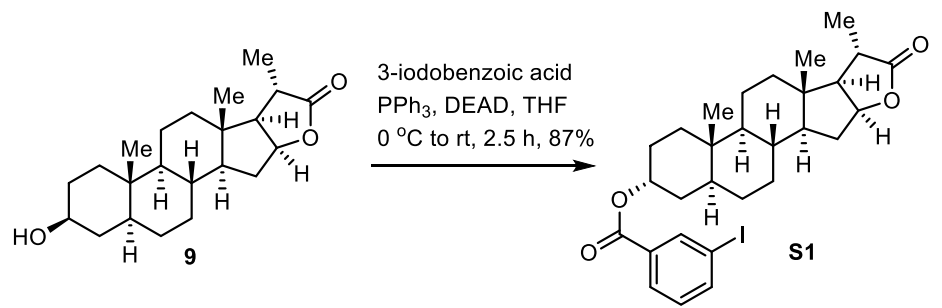

Compound 9 was synthesized from commercially available tigogenin (CAS: 77-60-1) according to a procedure reported by Tian and co-workers. ${ }^{1}$

To a solution of 9 (20.0 g, $57.8 \mathrm{mmol}, 1.0$ equiv), 3-iodobenzoic acid (17.2 g, 69.4 mmol, 1.2 equiv) and $\mathrm{PPh}_{3}(22.8 \mathrm{~g}, 86.7 \mathrm{mmol}, 1.5$ equiv $)$ in $300 \mathrm{~mL}$ dry THF was added DEAD (13.6 mL, $86.7 \mathrm{mmol}, 1.5$ equiv) dropwise at $0{ }^{\circ} \mathrm{C}$. The reaction mixture was stirred at room temperature for $2.5 \mathrm{~h}$ and quenched by $10 \mathrm{~mL} \mathrm{H}_{2} \mathrm{O}$. THF was removed under reduced pressure and the resulting mixture was treated with $260 \mathrm{~mL}$ EtOH. Stirring was continued for $2.5 \mathrm{~h}$ at room temperature to afford a suspension which was filtered, washed with $100 \mathrm{~mL}$ EtOH twice. The filter cake was dried using an infrared lamp to afford compound S1 $(20.0 \mathrm{~g}, 60 \%)$ as a white solid, which is sufficiently pure for direct use in the next step.

The mother liquor was concentrated under reduced pressure to give a brown oil, which was dissolved in $300 \mathrm{~mL}$ EtOAc, and filtered to remove diethyl hydrazodicarboxylate. Then $\mathrm{ZnCl}_{2}(15.0 \mathrm{~g}, 110.0 \mathrm{mmol}, 1.9$ equiv $)$ was added to the filtrate to give a suspension, which was filtered to remove another by-product triphenylphosphine oxide. The mother liquor was concentrated under reduced pressure and purification by flash chromatography ( $\mathrm{SiO}_{2}$, 5:5:1 DCM:petroleum ether:EtOAc) provided compound S1 $(9.0 \mathrm{~g}, 27 \%)$ as a white solid.

Compound S1: mp: $192.1-194.4{ }^{\circ} \mathrm{C}$; TLC (petroleum ether:EtOAc, $2: 1 \mathrm{v} / \mathrm{v}$ ): $R_{f}=$ $0.85 ;[\alpha]_{\mathrm{D}}^{30}-16.3\left(c 1.00, \mathrm{CHCl}_{3}\right) ;{ }^{1} \mathrm{H}$ NMR $\left(400 \mathrm{MHz}, \mathrm{CDCl}_{3}\right) \delta 8.34(\mathrm{t}, J=1.7 \mathrm{~Hz}$, $1 \mathrm{H}), 8.00(\mathrm{dt}, J=7.8,1.4 \mathrm{~Hz}, 1 \mathrm{H}), 7.87$ (dt, $J=7.9,1.4 \mathrm{~Hz}, 1 \mathrm{H}), 7.18(\mathrm{t}, J=7.8 \mathrm{~Hz}$, 1H), $5.28-5.21(\mathrm{~m}, 1 \mathrm{H}), 4.93(\mathrm{td}, J=7.8,4.6 \mathrm{~Hz}, 1 \mathrm{H}), 2.57(\mathrm{q}, J=7.6 \mathrm{~Hz}, 1 \mathrm{H}), 2.26$ $(\mathrm{dt}, J=13.9,7.3 \mathrm{~Hz}, 1 \mathrm{H}), 1.89-1.81(\mathrm{~m}, 2 \mathrm{H}), 1.80-1.63(\mathrm{~m}, 4 \mathrm{H}), 1.62-1.43(\mathrm{~m}$, 
7H), $1.37-1.18(\mathrm{~m}, 4 \mathrm{H}), 1.30(\mathrm{~d}, J=7.6 \mathrm{~Hz}, 3 \mathrm{H}), 1.17-1.05(\mathrm{~m}, 2 \mathrm{H}), 1.03-0.90(\mathrm{~m}$, 1H), 0.84 (s, 3H), 0.74 (s, 3H); ${ }^{13} \mathrm{C}$ NMR (101 MHz, $\left.\mathrm{CDCl}_{3}\right) \delta 181.4,164.5,141.7$, $138.5,133.1,130.1,128.8,93.9,82.9,71.4,59.2,54.7,54.4,41.8,40.5,38.4,36.2,36.1$, $35.0,33.2,33.1,32.9,32.1,28.2,26.3,20.3,18.1,14.0,11.5$; IR $(\mathrm{KBr}): v=2932,2853$, 1769, 1714, 1263, 1182, 973, $747 \mathrm{~cm}^{-1}$; HRMS (ESI, $\left.m / z\right)$ : $[\mathrm{M}+\mathrm{H}]^{+}$calcd for $\mathrm{C}_{29} \mathrm{H}_{38} \mathrm{IO}_{4}$, 577.1809; found, 577.1800.

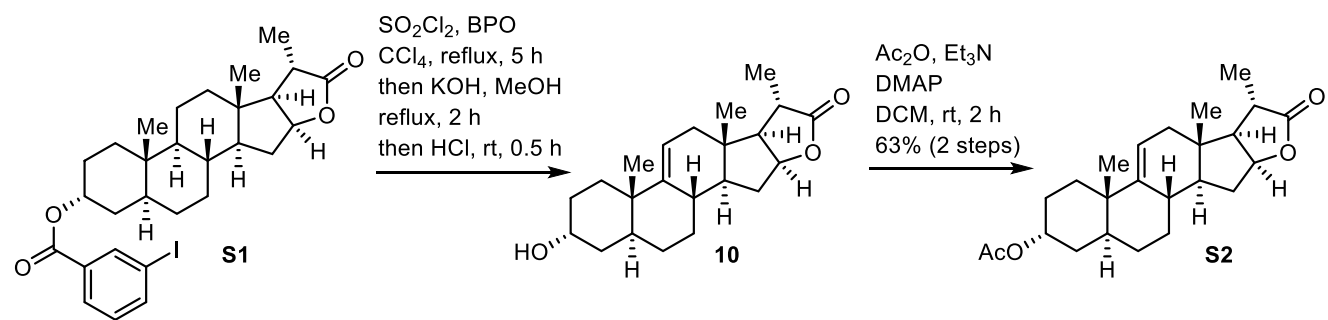

To a solution of $\mathbf{S 1}$ (20.0 g, $34.7 \mathrm{mmol}, 1.0$ equiv) and BPO (1.7 g, $6.9 \mathrm{mmol}, 0.2$ equiv) in $700 \mathrm{~mL}$ dry $\mathrm{CCl}_{4}$ was added $\mathrm{SO}_{2} \mathrm{Cl}_{2}(4.5 \mathrm{~mL}, 55.6 \mathrm{mmol}, 1.6$ equiv) under argon. The solution was refluxed for $5 \mathrm{~h}$ before cooled to room temperature, and concentration under reduced pressure afforded a yellow foam. The crude mixture was dissolved in $300 \mathrm{~mL} \mathrm{MeOH}$ and a solution of $\mathrm{KOH}(36.0 \mathrm{~g}, 642.9 \mathrm{mmol}, 18.5$ equiv) in $300 \mathrm{~mL}$ $\mathrm{MeOH}$ was added. The reaction mixture was refluxed for $2 \mathrm{~h}$ before cooled to room temperature. Concentration under reduced pressure afforded the crude reaction mixture which was neutralized with aq. $\mathrm{HCl}(2.4 \mathrm{M}, 300 \mathrm{~mL})$ to $\mathrm{pH}$ 2-3. Stirring was continued for $0.5 \mathrm{~h}$ at room temperature and the reaction mixture was extracted with EtOAc $(2 \times 150 \mathrm{~mL})$. The combined organic layers were washed successively with sat. aq. $\mathrm{NaHCO}_{3}(2 \times 200 \mathrm{~mL}), \mathrm{H}_{2} \mathrm{O}(200 \mathrm{~mL})$, brine $(50 \mathrm{~mL})$ and dried over $\mathrm{Na}_{2} \mathrm{SO}_{4}$. Removal of the solvent under reduced pressure afforded the crude product $\mathbf{1 0}$ which was used in the next step without further purification.

To a solution of the crude $\mathbf{1 0}$ obtained above in $150 \mathrm{~mL}$ dry DCM was added DMAP (690.0 mg, 5.7 mmol, 0.16 equiv), $\mathrm{Et}_{3} \mathrm{~N}$ (6.3 mL, $45.3 \mathrm{mmol}, 1.3$ equiv) and $\mathrm{Ac}_{2} \mathrm{O}$ (4.3 $\mathrm{mL}, 45.3 \mathrm{mmol}, 1.3$ equiv) at room temperature. After stirred at this temperature for 2 $\mathrm{h}$, the reaction mixture was quenched with $\mathrm{H}_{2} \mathrm{O}(50 \mathrm{~mL})$ and extracted with DCM 
$(3 \times 100 \mathrm{~mL})$. The combined organic layers were washed successively with sat. aq. $\mathrm{NaHCO}_{3}(200 \mathrm{~mL}), \mathrm{H}_{2} \mathrm{O}(200 \mathrm{~mL})$, brine $(200 \mathrm{~mL})$, dried over $\mathrm{Na}_{2} \mathrm{SO}_{4}$ and concentrated in vacuo. The resulting residue was treated with a mixed solvent of petroleum ether and ${ }^{i} \mathrm{BuOH}(35 \mathrm{~mL}, 3: 1, \mathrm{v} / \mathrm{v})$. The suspension was stirred at room temperature for $2 \mathrm{~h}$, filtered, and the filter cake was washed with petroleum ether and ${ }^{i} \mathrm{BuOH}(30 \mathrm{~mL}, 3: 1, \mathrm{v} / \mathrm{v})$. The residue was dried using an infrared lamp to afford $\mathbf{S 2}$ (8.5 g, 63\% over 2 steps) as a light yellow solid, which was sufficiently pure for direct use in the next step.

Compound 10: mp: $214.0-216.0^{\circ} \mathrm{C}$; TLC (petroleum ether:EtOAc, $2: 1 \mathrm{v} / \mathrm{v}$ ): $R_{f}=0.31$; $[\alpha]_{\mathrm{D}}^{30}-30.4\left(c 1.00, \mathrm{CHCl}_{3}\right) ;{ }^{1} \mathrm{H} \mathrm{NMR}\left(400 \mathrm{MHz}, \mathrm{CDCl}_{3}\right) \delta 5.29(\mathrm{~d}, J=5.8 \mathrm{~Hz}, 1 \mathrm{H})$, $4.95(\mathrm{td}, J=7.7,4.4 \mathrm{~Hz}, 1 \mathrm{H}), 4.04-4.00(\mathrm{~m}, 1 \mathrm{H}), 2.61(\mathrm{q}, J=7.6 \mathrm{~Hz}, 1 \mathrm{H}), 2.37(\mathrm{dt}, J$ $=14.3,7.5 \mathrm{~Hz}, 1 \mathrm{H}), 2.13-2.04(\mathrm{~m}, 1 \mathrm{H}), 2.02-1.95(\mathrm{~m}, 1 \mathrm{H}), 1.95-1.84(\mathrm{~m}, 3 \mathrm{H}), 1.75$ $-1.67(\mathrm{~m}, 2 \mathrm{H}), 1.65-1.54(\mathrm{~m}, 2 \mathrm{H}), 1.54-1.35(\mathrm{~m}, 5 \mathrm{H}), 1.34-1.21(\mathrm{~m}, 3 \mathrm{H}), 1.29(\mathrm{~d}$, $J=7.6 \mathrm{~Hz}, 3 \mathrm{H}), 1.06-0.96(\mathrm{~m}, 1 \mathrm{H}), 0.88(\mathrm{~s}, 3 \mathrm{H}), 0.66(\mathrm{~s}, 3 \mathrm{H}) ;{ }^{13} \mathrm{C} \mathrm{NMR}(101 \mathrm{MHz}$, $\left.\mathrm{CDCl}_{3}\right) \delta 181.3,147.5,114.7,83.2,66.3,58.8,52.4,40.3,40.0,38.5,37.7,36.5,36.0$ (2C), 34.1, 33.2, 30.7, 29.1, 28.2, 17.9, 16.9, 13.4; IR (KBr): $v=3521,2917,2856$, 1754, 1196, 1004, 963, $918 \mathrm{~cm}^{-1}$; HRMS (ESI, $\left.m / z\right)$ : $[\mathrm{M}+\mathrm{Na}]^{+}$calcd for $\mathrm{C}_{22} \mathrm{H}_{32} \mathrm{O}_{3} \mathrm{Na}$, 367.2244; found, 367.2240 .

Compound S2: mp: $121.1-123.5^{\circ} \mathrm{C}$; TLC (petroleum ether:EtOAc, $2: 1 \mathrm{v} / \mathrm{v}$ ): $R_{f}=$ $0.88 ;[\alpha]_{\mathrm{D}}^{27}-14.6\left(c 1.00, \mathrm{CHCl}_{3}\right) ;{ }^{1} \mathrm{H} \mathrm{NMR}\left(400 \mathrm{MHz}, \mathrm{CDCl}_{3}\right) \delta 5.30(\mathrm{~d}, J=5.8 \mathrm{~Hz}$, 1H), $5.02-4.93(\mathrm{~m}, 2 \mathrm{H}), 2.62(\mathrm{q}, J=7.6 \mathrm{~Hz}, 1 \mathrm{H}), 2.39(\mathrm{dt}, J=14.0,7.4 \mathrm{~Hz}, 1 \mathrm{H}), 2.15$ $-2.06(\mathrm{~m}, 1 \mathrm{H}), 2.05-1.97(\mathrm{~m}, 1 \mathrm{H}), 2.00(\mathrm{~s}, 3 \mathrm{H}), 1.96-1.87(\mathrm{~m}, 3 \mathrm{H}), 1.86-1.78(\mathrm{~m}$, 1H), $1.76-1.59(\mathrm{~m}, 2 \mathrm{H}), 1.57-1.45(\mathrm{~m}, 5 \mathrm{H}), 1.37-1.25(\mathrm{~m}, 3 \mathrm{H}), 1.30(\mathrm{~d}, J=7.6 \mathrm{~Hz}$, $3 \mathrm{H}), 1.00(\mathrm{qd}, J=12.8,4.9 \mathrm{~Hz}, 1 \mathrm{H}), 0.91(\mathrm{~s}, 3 \mathrm{H}), 0.67(\mathrm{~s}, 3 \mathrm{H}) ;{ }^{13} \mathrm{C} \mathrm{NMR}(101 \mathrm{MHz}$, $\left.\mathrm{CDCl}_{3}\right) \delta 181.2,170.7,147.5,114.9,83.1,69.9,58.9,52.4,40.4,40.0,38.7,38.3,36.5$, $36.1,34.1,33.14,33.11,31.5,28.0,26.3,21.6,17.9,17.1,13.4$; IR $(\mathrm{KBr}): v=2933$, 2873, 1768, 1723, 1240, 1182, 1025, $968 \mathrm{~cm}^{-1}$; HRMS (ESI, $\left.m / z\right):[\mathrm{M}+\mathrm{H}]^{+}$calcd for $\mathrm{C}_{24} \mathrm{H}_{35} \mathrm{O}_{4}, 387.2530$; found, 387.2532. 


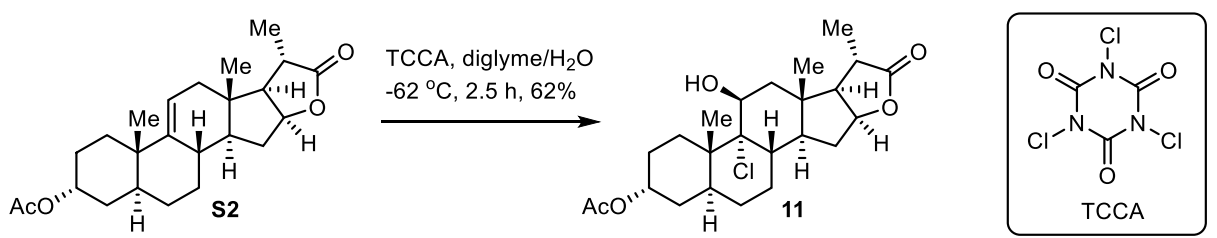

To a solution of $\mathbf{S 2}(5.0 \mathrm{~g}, 13.0 \mathrm{mmol}, 1.0$ equiv) in diglyme (diethylene glycol dimethyl ether $) / \mathrm{H}_{2} \mathrm{O}(600 \mathrm{~mL}, 9: 1, \mathrm{v} / \mathrm{v})$ was added a solution of TCCA (2.5 g, $10.7 \mathrm{mmol}, 0.8$ equiv) in $5 \mathrm{~mL}$ diglyme at $-62^{\circ} \mathrm{C}$. After stirred at the same temperature for $2.5 \mathrm{~h}$, the reaction mixture was quenched with sat. aq. $\mathrm{Na}_{2} \mathrm{~S}_{2} \mathrm{O}_{3}(40 \mathrm{~mL})$ and warmed to room temperature. Stirring was continued for another $10 \mathrm{~h}$ until the solution became clear. The reaction mixture was extracted with EtOAc $(3 \times 200 \mathrm{~mL})$ and the combined organic layers were washed with aq. $\mathrm{NaCl}$ (sat. aq. $\mathrm{NaCl} / \mathrm{H}_{2} \mathrm{O}, 1: 1,3 \times 200 \mathrm{~mL}$ ) and brine. Concentration under reduced pressure afforded the crude product, which was purified by flash chromatography $\left(\mathrm{SiO}_{2}, 7: 7: 1\right.$ petroleum ether:DCM:EtOAc) to provide compound $11(3.6 \mathrm{~g}, 62 \%)$ as a white solid.

Compound 11: mp: $180.9-184.1{ }^{\circ} \mathrm{C}$; TLC (petroleum ether:acetone, $4: 1 \mathrm{v} / \mathrm{v}$ ): $R_{f}=$ 0.37; $[\alpha]_{\mathrm{D}}^{27}-7.4\left(c 0.65, \mathrm{CHCl}_{3}\right) ;{ }^{1} \mathrm{H} \mathrm{NMR}\left(400 \mathrm{MHz}, \mathrm{CDCl}_{3}\right) \delta 5.00-4.90(\mathrm{~m}, 2 \mathrm{H})$, $4.41-4.37(\mathrm{~m}, 1 \mathrm{H}), 2.58(\mathrm{q}, J=7.6 \mathrm{~Hz}, 1 \mathrm{H}), 2.53-2.41(\mathrm{~m}, 2 \mathrm{H}), 2.28-2.20(\mathrm{~m}, 1 \mathrm{H})$, $2.19-2.09(\mathrm{~m}, 1 \mathrm{H}), 2.05(\mathrm{~s}, 3 \mathrm{H}), 1.94(\mathrm{~d}, J=7.8 \mathrm{~Hz}, 1 \mathrm{H}), 1.92-1.84(\mathrm{~m}, 1 \mathrm{H}), 1.82-$ $1.73(\mathrm{~m}, 3 \mathrm{H}), 1.72-1.67(\mathrm{~m}, 1 \mathrm{H}), 1.66-1.59(\mathrm{~m}, 1 \mathrm{H}), 1.58-1.47(\mathrm{~m}, 4 \mathrm{H}), 1.46-$ $1.35(\mathrm{~m}, 2 \mathrm{H}), 1.30(\mathrm{~d}, J=7.6 \mathrm{~Hz}, 3 \mathrm{H}), 1.27$ (s, 3H), $1.26-1.19(\mathrm{~m}, 2 \mathrm{H}), 0.99$ (s, 3H); ${ }^{13} \mathrm{C}$ NMR $\left(101 \mathrm{MHz}, \mathrm{CDCl}_{3}\right) \delta 181.5,170.9,91.2,82.5,73.7,69.5,59.2,48.2,43.1$, $42.2,41.3,36.1,35.3,33.0,32.5,32.4,27.4,27.2,26.7,25.7,21.6,18.1,17.6,16.5$; IR $(\mathrm{KBr}): v=3463,2936,2879,1748,1730,1265,1027,736 \mathrm{~cm}^{-1}$; HRMS (ESI, $\left.\mathrm{m} / z\right)$ ): $[\mathrm{M}+\mathrm{H}]^{+}$calcd for $\mathrm{C}_{24} \mathrm{H}_{36} \mathrm{ClO}_{5}, 439.2246$; found, 439.2252 .

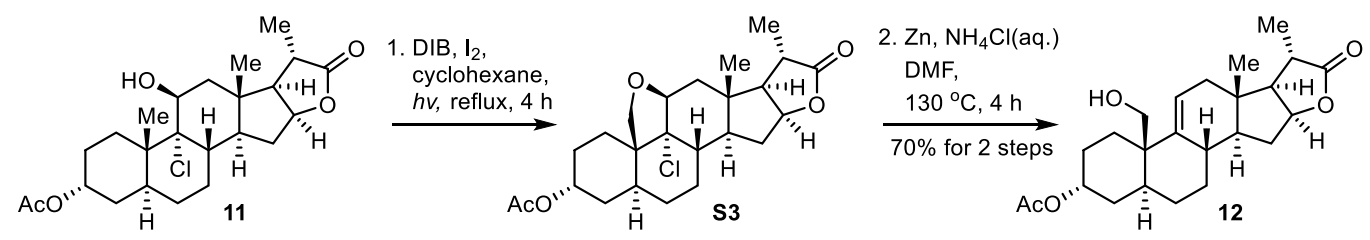


To a solution of 11 ( $8.0 \mathrm{~g}, 18.2 \mathrm{mmol}, 1.0$ equiv) and DIB (11.7 g, $36.4 \mathrm{mmol}, 2.0$ equiv) in $800 \mathrm{~mL}$ dry cyclohexane was added $\mathrm{I}_{2}$ (9.2 g, 36.4 mmol, 2.0 equiv). The reaction mixture was heated to reflux by irradiation with an infrared lamp (275 W) for $2 \mathrm{~h}$. Another portion of DIB (5.9 g, $18.2 \mathrm{mmol}, 1.0$ equiv) and $\mathrm{I}_{2}$ (4.6 g, $18.2 \mathrm{mmol}, 1.0$ equiv) was added and stirring was continued for another $2 \mathrm{~h}$ under the same condition. The reaction mixture was cooled to room temperature, quenched with sat. aq. $\mathrm{Na}_{2} \mathrm{~S}_{2} \mathrm{O}_{3}$ $(100 \mathrm{~mL})$, and extracted with EtOAc $(3 \times 200 \mathrm{~mL})$. The combined organic layers were washed with brine, dried over $\mathrm{Na}_{2} \mathrm{SO}_{4}$. Removal of the solvent under reduced pressure afforded the crude product, which was purified by flash chromatography $\left(\mathrm{SiO}_{2}\right.$, 13:5:1 $\rightarrow$ 3:1:1 petroleum ether:DCM:EtOAc) to provide crude $\mathbf{S 3}$ (7.5 g).

To a solution of $\mathbf{S 3}$ (7.5 g, $17.2 \mathrm{mmol}, 1.0$ equiv) in $113 \mathrm{~mL} \mathrm{DMF}$ and $47 \mathrm{~mL}$ sat. aq. $\mathrm{NH}_{4} \mathrm{Cl}$ was added zinc powder $\left(16.7 \mathrm{~g}, 257.4 \mathrm{mmol}, 15.0\right.$ equiv) and $\mathrm{NH}_{4} \mathrm{Cl}_{(\mathrm{s})}(9.2 \mathrm{~g}$, $171.6 \mathrm{mmol}, 10.0$ equiv). After the reaction mixture was refluxed at $140{ }^{\circ} \mathrm{C}$ for $2 \mathrm{~h}$, another portion of zinc powder $(11.1 \mathrm{~g}, 171.6 \mathrm{mmol}, 10.0$ equiv) and $47 \mathrm{~mL}$ sat. aq. $\mathrm{NH}_{4} \mathrm{Cl}$ was added. After stirred for $2 \mathrm{~h}$ under the same condition, the reaction mixture was cooled to room temperature and filtered through celite. The filtrate was extracted with EtOAc $(3 \times 200 \mathrm{~mL})$ and the combined organic layers were washed with brine and dried over $\mathrm{Na}_{2} \mathrm{SO}_{4}$. Removal of the solvent under reduced pressure afforded the crude product, which was purified by flash chromatography $\left(\mathrm{SiO}_{2}, 11: 3: 1 \rightarrow 3: 2: 1\right.$ petroleum ether:DCM:EtOAc) to provide 12 (5.1 g, 70\% for 2 steps) as a white solid.

Compound S3: mp: $251.3-255.6{ }^{\circ} \mathrm{C}$; TLC (petroleum ether:acetone, 4:1 v/v): $R_{f}=$ $0.41 ;[\alpha]_{\mathrm{D}}^{27}-10.3\left(c 0.54, \mathrm{CHCl}_{3}\right) ;{ }^{1} \mathrm{H} \mathrm{NMR}\left(400 \mathrm{MHz}, \mathrm{CDCl}_{3}\right) \delta 5.03-4.09(\mathrm{~m}, 1 \mathrm{H})$, $4.97(\mathrm{td}, J=7.8,4.7 \mathrm{~Hz}, 1 \mathrm{H}), 4.19(\mathrm{t}, J=3.0 \mathrm{~Hz}, 1 \mathrm{H}), 3.94(\mathrm{dd}, J=8.7,1.9 \mathrm{~Hz}, 1 \mathrm{H})$, $3.88(\mathrm{~d}, J=8.7 \mathrm{~Hz}, 1 \mathrm{H}), 2.63(\mathrm{q}, J=7.6 \mathrm{~Hz}, 1 \mathrm{H}), 2.20(\mathrm{dt}, J=14.0,7.2 \mathrm{~Hz}, 1 \mathrm{H}), 2.07$ (s, 3H), $2.05(\mathrm{~d}, J=1.9 \mathrm{~Hz}, 1 \mathrm{H}), 2.01(\mathrm{~d}, J=2.2 \mathrm{~Hz}, 1 \mathrm{H}), 1.97-1.85(\mathrm{~m}, 4 \mathrm{H}), 1.74$ (dt, $J=15.0,2.7 \mathrm{~Hz}, 1 \mathrm{H}), 1.70-1.63(\mathrm{~m}, 2 \mathrm{H}), 1.62-1.43(\mathrm{~m}, 6 \mathrm{H}), 1.31(\mathrm{~d}, J=7.6 \mathrm{~Hz}$, $3 \mathrm{H}), 1.35-1.23(\mathrm{~m}, 1 \mathrm{H}), 1.17(\mathrm{td}, J=14.2,2.8 \mathrm{~Hz}, 1 \mathrm{H}), 0.94(\mathrm{~s}, 3 \mathrm{H}) ;{ }^{13} \mathrm{C}$ NMR $(101$ $\left.\mathrm{MHz}, \mathrm{CDCl}_{3}\right) \delta 181.0,170.7,82.6,80.3,78.1,68.9,67.7,59.4,49.1,45.8,42.9,38.5$, 
36.9, 36.3, 35.0, 32.1, 31.9, 30.2, 27.6, 26.6, 24.0, 21.6, 18.1, 16.3; IR (KBr): $v=2938$, 2882, 1761, 1738, 1256, 1235, 947, $735 \mathrm{~cm}^{-1}$; HRMS (ESI, $\left.\mathrm{m} / z\right):[\mathrm{M}+\mathrm{H}]^{+}$calcd for $\mathrm{C}_{24} \mathrm{H}_{34} \mathrm{ClO}_{5}, 437.2089$; found, 437.2090.

Compound 12: mp: $195.3-197.7^{\circ} \mathrm{C}$; TLC (petroleum ether:EtOAc, $1: 1 \mathrm{v} / \mathrm{v}$ ): $R_{f}=0.35$; $[\alpha]_{\mathrm{D}}^{27}-10.4\left(c 0.73, \mathrm{CHCl}_{3}\right) ;{ }^{1} \mathrm{H} \mathrm{NMR}\left(400 \mathrm{MHz}, \mathrm{CDCl}_{3}\right) \delta 5.47(\mathrm{~d}, J=5.3 \mathrm{~Hz}, 1 \mathrm{H})$, $5.11-5.03(\mathrm{~m}, 1 \mathrm{H}), 4.99(\mathrm{td}, J=7.7,4.3 \mathrm{~Hz}, 1 \mathrm{H}), 3.73-3.63(\mathrm{~m}, 2 \mathrm{H}), 2.65(\mathrm{q}, J=7.6$ Hz, 1H), 2.43 (dt, $J=14.4,7.4 \mathrm{~Hz}, 1 \mathrm{H}), 2.21-2.08$ (m, 2H), 2.04 (s, 3H), $2.03-1.94$ (m, 3H), $1.94-1.70(\mathrm{~m}, 4 \mathrm{H}), 1.59-1.49(\mathrm{~m}, 3 \mathrm{H}), 1.46-1.29(\mathrm{~m}, 4 \mathrm{H}), 1.34(\mathrm{~d}, J=7.6$ $\mathrm{Hz}, 3 \mathrm{H}), 1.11(\mathrm{qd}, J=12.7,4.5 \mathrm{~Hz}, 1 \mathrm{H}), 0.84-0.77(\mathrm{~m}, 1 \mathrm{H}), 0.72(\mathrm{~s}, 3 \mathrm{H}) ;{ }^{13} \mathrm{C} \mathrm{NMR}$ $\left(101 \mathrm{MHz}, \mathrm{CDCl}_{3}\right) \delta 181.0,170.7,142.3,120.8,82.9,69.5,58.9,58.1,52.4,43.8,40.3$, $40.2,38.5,36.5,35.9,34.1,33.1,32.8,27.5,25.9,25.0,21.6,18.0,14.0$; IR $(\mathrm{KBr}): v=$ 3527, 2932, 2871, 1768, 1731, 1256, 962, $734 \mathrm{~cm}^{-1}$; HRMS (ESI, $\left.m / z\right):[\mathrm{M}+\mathrm{H}]^{+}$calcd for $\mathrm{C}_{24} \mathrm{H}_{35} \mathrm{O}_{5}, 403.2479$; found, 403.2482 .

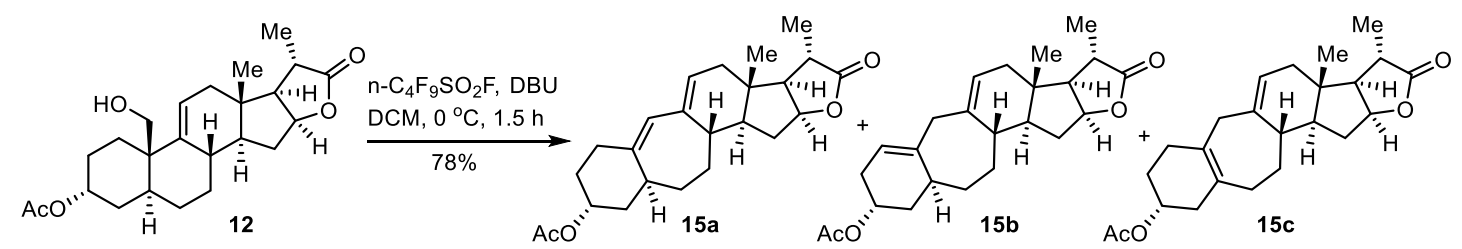

To a stirred solution of 12 (100.0 mg, $0.25 \mathrm{mmol}, 1.0$ equiv) and DBU (148 $\mu \mathrm{L}, 1.00$ mmol, 4.0 equiv) in $5 \mathrm{~mL}$ dry DCM was added $n-\mathrm{C}_{4} \mathrm{~F}_{9} \mathrm{SO}_{2} \mathrm{~F}(133 \mu \mathrm{L}, 0.75 \mathrm{mmol}, 3.0$ equiv) at $0{ }^{\circ} \mathrm{C}$. The reaction mixture was stirred at the same temperature for $1.5 \mathrm{~h}$, quenched with sat. aq. $\mathrm{NH}_{4} \mathrm{Cl}(5 \mathrm{~mL})$ and extracted with $\mathrm{DCM}(3 \times 10 \mathrm{~mL})$. The combined organic layers were washed with brine and dried over $\mathrm{Na}_{2} \mathrm{SO}_{4}$. Removal of the solvent under reduced pressure afforded the crude product which was purified by flash chromatography $\left(\mathrm{SiO}_{2}, 10: 1\right.$ petroleum ether: EtOAc) to afford a 1.4:2.2:1.0 mixture of 15a, 15b and $\mathbf{1 5 c}$ in $78 \%$ combined yield $(75.0 \mathrm{mg})$.

Compound 15a: white foam; TLC (petroleum ether:EtOAc, $2: 1 \mathrm{v} / \mathrm{v}$ ): $R_{f}=0.73 ;[\alpha]_{\mathrm{D}}^{25}$ -47.4 (c 1.00, $\left.\mathrm{CHCl}_{3}\right) ;{ }^{1} \mathrm{H}$ NMR (400 MHz, $\left.\mathrm{CDCl}_{3}\right) \delta 5.89(\mathrm{~s}, 1 \mathrm{H}), 5.56$ (d, $J=5.2 \mathrm{~Hz}$, 1H), $5.12-5.07(\mathrm{~m}, 1 \mathrm{H}), 4.98(\mathrm{td}, J=7.7,4.2 \mathrm{~Hz}, 1 \mathrm{H}), 2.66(\mathrm{q}, J=7.8 \mathrm{~Hz}, 1 \mathrm{H}), 2.53$ 
$-2.33(\mathrm{~m}, 3 \mathrm{H}), 2.20-1.96(\mathrm{~m}, 6 \mathrm{H}), 2.06(\mathrm{~s}, 3 \mathrm{H}), 1.96-1.87(\mathrm{~m}, 2 \mathrm{H}), 1.77$ (dd, $J=$ 12.7, $7.8 \mathrm{~Hz}, 1 \mathrm{H}), 1.67-1.59(\mathrm{~m}, 1 \mathrm{H}), 1.53-1.42(\mathrm{~m}, 1 \mathrm{H}), 1.38-1.19(\mathrm{~m}, 4 \mathrm{H}), 1.33$ $(\mathrm{d}, J=7.6 \mathrm{~Hz}, 3 \mathrm{H}), 0.79$ (s, 3H); ${ }^{13} \mathrm{C} \mathrm{NMR}\left(101 \mathrm{MHz}, \mathrm{CDCl}_{3}\right) \delta 181.0,170.8,139.2$, $138.6,128.3,127.3,82.9,69.8,59.0,51.0,44.1,40.8,40.7,39.2,37.3,37.0,36.7,34.5$, 34.3, 31.2, 29.3, 21.6, 17.9, 14.2; IR (KBr): $v=2930,2852,1771,1733,1249,1180$, 1037, $734 \mathrm{~cm}^{-1}$; HRMS (ESI, $\mathrm{m} / z$ ): $[\mathrm{M}+\mathrm{Na}]^{+}$calcd for $\mathrm{C}_{24} \mathrm{H}_{32} \mathrm{O}_{4} \mathrm{Na}, 407.2193$; found, 407.2192.

Compound 15b: mp: $185.2-187.1{ }^{\circ} \mathrm{C}$; TLC (petroleum ether:EtOAc, $2: 1 \mathrm{v} / \mathrm{v}$ ): $R_{f}=$ $0.73 ;[\alpha]_{\mathrm{D}}^{25}-137.5\left(c 0.87, \mathrm{CHCl}_{3}\right) ;{ }^{1} \mathrm{H} \mathrm{NMR}\left(400 \mathrm{MHz}, \mathrm{CDCl}_{3}\right) \delta 5.33$ (brs, 1H), 5.27 $(\mathrm{d}, J=2.8 \mathrm{~Hz}, 1 \mathrm{H}), 5.06-4.93(\mathrm{~m}, 2 \mathrm{H}), 2.97(\mathrm{~d}, J=15.4 \mathrm{~Hz}, 1 \mathrm{H}), 2.87(\mathrm{~d}, J=15.3$ $\mathrm{Hz}, 1 \mathrm{H}), 2.64(\mathrm{q}, J=7.5 \mathrm{~Hz}, 1 \mathrm{H}), 2.46-2.36(\mathrm{~m}, 2 \mathrm{H}), 2.36-2.26(\mathrm{~m}, 1 \mathrm{H}), 2.04(\mathrm{~s}$, $3 \mathrm{H}), 2.01-1.95(\mathrm{~m}, 2 \mathrm{H}), 1.95-1.81(\mathrm{~m}, 3 \mathrm{H}), 1.80-1.69(\mathrm{~m}, 3 \mathrm{H}), 1.61-1.51(\mathrm{~m}$, 2H), $1.50-1.35$ (m, 3H), 1.32 (d, J=7.6 Hz, 3H), 0.73 (s, 3H); ${ }^{13} \mathrm{C}$ NMR (101 MHz, $\left.\mathrm{CDCl}_{3}\right) \delta 181.1,171.0,139.8,139.2,119.9,119.3,82.8,68.1,59.1,50.6,43.7,41.4$, $40.8,40.0,39.8,36.6,35.8,34.7,34.2,31.7,29.5,21.6,18.0,13.9$; IR (KBr): $v=2923$, 2853, 1770, 1727, 1245, 1033, 804, $734 \mathrm{~cm}^{-1}$; HRMS (ESI, $\left.m / z\right):[\mathrm{M}+\mathrm{Na}]^{+}$calcd for $\mathrm{C}_{24} \mathrm{H}_{32} \mathrm{O}_{4} \mathrm{Na}$, 407.2193; found, 407.2191.

Compound 15c: colorless oil; TLC (petroleum ether:EtOAc, $2: 1 \mathrm{v} / \mathrm{v}$ ): $R_{f}=0.73 ;[\alpha]_{\mathrm{D}}^{25}$ $-4.4\left(c\right.$ 0.50, $\left.\mathrm{CHCl}_{3}\right) ;{ }^{1} \mathrm{H} \mathrm{NMR}\left(400 \mathrm{MHz}, \mathrm{CDCl}_{3}\right) \delta 5.30(\mathrm{~d}, J=4.7 \mathrm{~Hz}, 1 \mathrm{H}), 5.01-$ $4.90(\mathrm{~m}, 2 \mathrm{H}), 2.94(\mathrm{~d}, J=14.9 \mathrm{~Hz}, 1 \mathrm{H}), 2.64$ (q, $J=7.6 \mathrm{~Hz}, 1 \mathrm{H}), 2.42$ (d, $J=14.9 \mathrm{~Hz}$, 1H), 2.39 (ddd, $J=13.2,7.6,6.0 \mathrm{~Hz}, 1 \mathrm{H}), 2.27-2.07(\mathrm{~m}, 5 \mathrm{H}), 2.03(\mathrm{~s}, 3 \mathrm{H}), 2.01-$ $1.84(\mathrm{~m}, 5 \mathrm{H}), 1.83-1.74(\mathrm{~m}, 1 \mathrm{H}), 1.73-1.62(\mathrm{~m}, 1 \mathrm{H}), 1.61-1.41(\mathrm{~m}, 3 \mathrm{H}), 1.32(\mathrm{~d}, J$ $=7.6 \mathrm{~Hz}, 3 \mathrm{H}), 1.29-1.20(\mathrm{~m}, 1 \mathrm{H}), 0.76(\mathrm{~s}, 3 \mathrm{H}) ;{ }^{13} \mathrm{C} \mathrm{NMR}\left(101 \mathrm{MHz}, \mathrm{CDCl}_{3}\right) \delta 181.2$, 171.0, 137.5, 130.1, 129.2, 121.1, 83.0, 70.2, 59.0, 50.6, 42.5, 41.7, 41.1, 39.8, 37.0, 36.6, 34.0, 31.2, 30.3, 29.2, 27.8, 21.6, 18.0, 13.8; IR (KBr): $v=2923,2838,1770$, 1732, 1244, 1031, 801, $734 \mathrm{~cm}^{-1}$; HRMS (ESI, $\left.m / z\right)$ : $[\mathrm{M}+\mathrm{Na}]^{+}$calcd for $\mathrm{C}_{24} \mathrm{H}_{32} \mathrm{O}_{4} \mathrm{Na}$, 407.2193; found, 407.2191. 


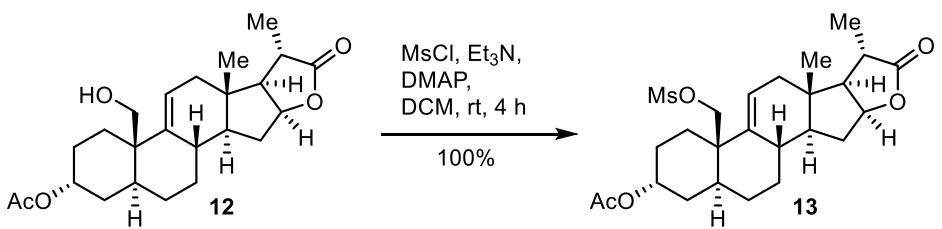

To a solution of 12 (3.6 g, $9.0 \mathrm{mmol}, 1.0$ equiv) and DMAP (110.0 mg, $0.9 \mathrm{mmol}, 0.1$ equiv) in $130 \mathrm{~mL}$ dry DCM was added $\mathrm{Et}_{3} \mathrm{~N}$ ( $3.7 \mathrm{~mL}, 27.0 \mathrm{mmol}, 3.0$ equiv) and $\mathrm{MsCl}$ (1.4 mL, $18.0 \mathrm{mmol}, 2.0$ equiv). The reaction mixture was stirred at room temperature for $4 \mathrm{~h}$ and concentrated in vacuo to provide the crude residue which was purified by flash chromatography $\left(\mathrm{SiO}_{2}, 8: 1 \rightarrow 5: 1\right.$ petroleum ehter:acetone) to provide $\mathbf{1 3}$ (4.3 g, $100 \%)$ as a white foam.

Compound 13: TLC (petroleum ether:EtOAc, $1: 1 \mathrm{v} / \mathrm{v}): R_{f}=0.35 ;[\alpha]_{\mathrm{D}}^{27}-11.5(c 1.00$, $\left.\mathrm{CHCl}_{3}\right) ;{ }^{1} \mathrm{H} \mathrm{NMR}\left(400 \mathrm{MHz}, \mathrm{CDCl}_{3}\right) \delta 5.45(\mathrm{~d}, J=5.8 \mathrm{~Hz}, 1 \mathrm{H}), 5.09-5.05(\mathrm{~m}, 1 \mathrm{H})$, $4.99(\mathrm{td}, J=7.6,4.3 \mathrm{~Hz}, 1 \mathrm{H}), 4.38(\mathrm{~d}, J=9.7 \mathrm{~Hz}, 1 \mathrm{H}), 4.33(\mathrm{~d}, J=9.7 \mathrm{~Hz}, 1 \mathrm{H}), 2.93$ (s, 3H), 2.64 (q, $J=7.4 \mathrm{~Hz}, 1 \mathrm{H}), 2.43$ (dt, $J=14.3,7.4 \mathrm{~Hz}, 1 \mathrm{H}), 2.18-2.08(\mathrm{~m}, 2 \mathrm{H})$, $2.04(\mathrm{~s}, 3 \mathrm{H}), 2.01-1.85(\mathrm{~m}, 5 \mathrm{H}), 1.82-1.71(\mathrm{~m}, 2 \mathrm{H}), 1.69-1.63(\mathrm{~m}, 1 \mathrm{H}), 1.59-1.48$ $(\mathrm{m}, 3 \mathrm{H}), 1.44-1.36(\mathrm{~m}, 3 \mathrm{H}), 1.33(\mathrm{~d}, J=7.7 \mathrm{~Hz}, 3 \mathrm{H}), 1.11(\mathrm{qd}, J=12.2,5.9 \mathrm{~Hz}, 1 \mathrm{H})$, $0.74(\mathrm{~s}, 3 \mathrm{H}) ;{ }^{13} \mathrm{C} \mathrm{NMR}\left(101 \mathrm{MHz}, \mathrm{CDCl}_{3}\right) \delta 181.0,170.5,140.5,120.7,82.9,69.0,65.9$, 58.7, 52.6, 41.6, 40.4, 40.1, 38.8, 37.4, 36.6, 35.8, 34.1, 33.1, 32.8, 27.6, 25.7, 25.1, 21.6, 17.9, 13.3; IR (KBr): $v=2936,2874,1766,1731,1449,1237,1175,735 \mathrm{~cm}^{-1}$; HRMS (ESI, $m / z$ ): $[\mathrm{M}+\mathrm{H}]^{+}$calcd for $\mathrm{C}_{25} \mathrm{H}_{37} \mathrm{O}_{7} \mathrm{~S}, 481.2255$; found, 481.2252 .

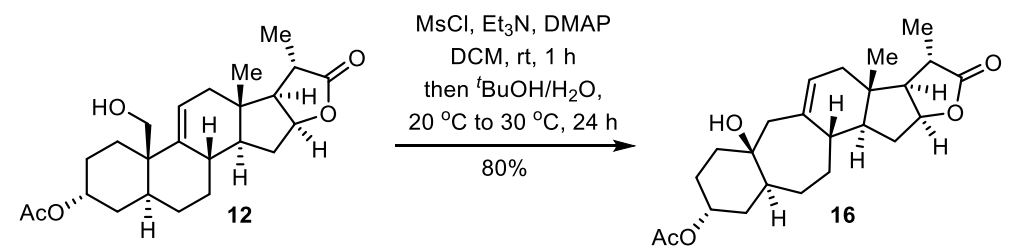

To a solution of 12 (4.0 g, 9.9 mmol, 1.0 equiv) and DMAP (242.8 mg, $2.0 \mathrm{mmol}, 0.2$ equiv) in $81 \mathrm{~mL}$ dry DCM was added $\mathrm{Et}_{3} \mathrm{~N}(4.14 \mathrm{~mL}, 29.8 \mathrm{mmol}, 3.0$ equiv) and $\mathrm{MsCl}$ (1.54 mL, $19.9 \mathrm{mmol}, 2.0$ equiv). The reaction mixture was stirred at room temperature for $1 \mathrm{~h}$ and concentrated in vacuo to provide the crude mesylate 13. After $\mathbf{1 3}$ was dissolved in $\mathrm{H}_{2} \mathrm{O} /{ }^{\circ} \mathrm{BuOH}\left(820 \mathrm{~mL}, 2: 1\right.$, v/v), the reaction mixture was stirred at $20{ }^{\circ} \mathrm{C}$ 
for $21 \mathrm{~h}$ and $30^{\circ} \mathrm{C}$ for $3 \mathrm{~h}$. The reaction mixture was extracted with EtOAc $(3 \times 200 \mathrm{~mL})$ and the combined organic layers were washed with brine and dried over $\mathrm{Na}_{2} \mathrm{SO}_{4}$. Removal of the solvent under reduced pressure afforded the crude residue which was purified by flash chromatography $\left(\mathrm{SiO}_{2}, 8: 1 \rightarrow 5: 1\right.$ petroleum ether: EtOAc) to furnish compound 16 (3.2 $\mathrm{g}, 80 \%)$ as a white solid.

Compound 16: mp: $177.1-179.5^{\circ} \mathrm{C}$; TLC (petroleum ether:EtOAc, $2: 1 \mathrm{v} / \mathrm{v}$ ): $R_{f}=0.51$; $[\alpha]_{\mathrm{D}}^{27}-49.0\left(c\right.$ 0.47, $\left.\mathrm{CHCl}_{3}\right) ;{ }^{1} \mathrm{H} \mathrm{NMR}\left(400 \mathrm{MHz}, \mathrm{CDCl}_{3}\right) \delta 5.35-5.30(\mathrm{~m}, 1 \mathrm{H}), 5.04$ $-5.00(\mathrm{~m}, 1 \mathrm{H}), 4.96(\mathrm{td}, J=7.7,4.2 \mathrm{~Hz}, 1 \mathrm{H}), 2.71-2.61(\mathrm{~m}, 2 \mathrm{H}), 2.40(\mathrm{dt}, J=14.0$, $7.4 \mathrm{~Hz}, 1 \mathrm{H}), 2.30(\mathrm{~d}, J=15.8 \mathrm{~Hz}, 1 \mathrm{H}), 2.18-2.09(\mathrm{~m}, 1 \mathrm{H}), 2.08-2.00(\mathrm{~m}, 1 \mathrm{H}), 2.04$ (s, 3H), $1.98(\mathrm{~d}, J=7.4 \mathrm{~Hz}, 1 \mathrm{H}), 1.90-1.72(\mathrm{~m}, 3 \mathrm{H}), 1.72-1.65(\mathrm{~m}, 3 \mathrm{H}), 1.63-1.52$ (m, 4H), $1.50-1.35(\mathrm{~m}, 3 \mathrm{H}), 1.34-1.29(\mathrm{~m}, 1 \mathrm{H}), 1.31(\mathrm{~d}, J=7.6 \mathrm{~Hz}, 3 \mathrm{H}), 1.06(\mathrm{~s}$, 1H), $0.78(\mathrm{~s}, 3 \mathrm{H}) ;{ }^{13} \mathrm{C} \mathrm{NMR}\left(101 \mathrm{MHz}, \mathrm{CDCl}_{3}\right) \delta 181.1,170.8,138.0,122.7,82.9,72.2$, $69.8,59.2,50.8,50.7,43.1,41.4,40.9,40.2,36.7,36.5,34.9,34.3,33.8,29.2,25.3$, 21.6, 17.9, 14.4; IR (KBr): $v=3496,2921,2874,1767,1731,1259,1240,735 \mathrm{~cm}^{-1}$; HRMS (ESI, $m / z)$ : [M-H] $]^{-}$calcd for $\mathrm{C}_{24} \mathrm{H}_{33} \mathrm{O}_{5}, 401.2333$; found, 401.2341 .

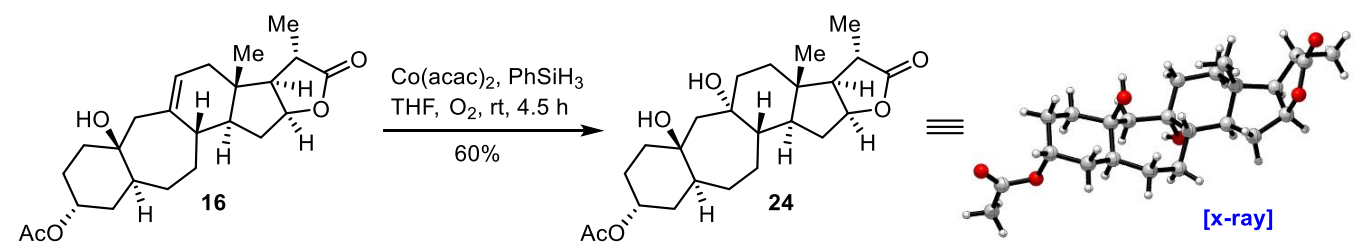

A stirred solution of 16 (300.0 mg, $0.75 \mathrm{mmol}, 1.0$ equiv) and $\mathrm{Co}(\mathrm{acac})_{2}(57.8 \mathrm{mg}, 0.23$ mmol, 0.3 equiv) in $21 \mathrm{~mL}$ dry THF was bubbled with $\mathrm{O}_{2}$ for $0.5 \mathrm{~h}$ at room temperature. Then $\mathrm{PhSiH}_{3}(0.37 \mathrm{~mL}, 3.00 \mathrm{mmol}, 4.0$ equiv) was added over $1 \mathrm{~h}$. Stirring was continued for $3 \mathrm{~h}$ at the same temperature under an $\mathrm{O}_{2}$ atmosphere (no bubbling). The reaction mixture was quenched with aq. $\mathrm{HCl}(1 \mathrm{M}, 5 \mathrm{~mL})$ and extracted with EtOAc $(3 \times 15 \mathrm{~mL})$. The combined organic layers were washed with brine and dried over $\mathrm{Na}_{2} \mathrm{SO}_{4}$. Removal of the solvent under reduced pressure afforded the crude residue, which was purified by flash chromatography $\left(\mathrm{SiO}_{2}, 2: 1 \rightarrow 1: 1\right.$ petroleum ether: EtOAc) to furnish compound $\mathbf{2 4}(187.0 \mathrm{mg}, 60 \%)$ as a white solid. 
The single crystal of compound $\mathbf{2 4}$ suitable for X-ray determination was obtained by slow evaporation of a solution of $\mathbf{2 4}$ in hexanes and acetone at room temperature.

Compound 24: mp: $141.0-143.0^{\circ} \mathrm{C}$; TLC (petroleum ether:EtOAc, $2: 1 \mathrm{v} / \mathrm{v}$ ): $R_{f}=0.14$; $[\alpha]_{\mathrm{D}}^{25}-30.4\left(c 1.00, \mathrm{CHCl}_{3}\right) ;{ }^{1} \mathrm{H} \mathrm{NMR}\left(400 \mathrm{MHz}, \mathrm{CDCl}_{3}\right) \delta 5.02-4.98(\mathrm{~m}, 1 \mathrm{H}), 4.94$ $(\mathrm{td}, J=7.7,4.6 \mathrm{~Hz}, 1 \mathrm{H}), 2.56(\mathrm{q}, J=7.4 \mathrm{~Hz}, 1 \mathrm{H}), 2.25(\mathrm{dt}, J=13.6,7.1 \mathrm{~Hz}, 1 \mathrm{H}), 2.03$ (s, 3H), $2.00-1.95(\mathrm{~m}, 1 \mathrm{H}), 1.91(\mathrm{~s}, 2 \mathrm{H}), 1.86-1.73(\mathrm{~m}, 4 \mathrm{H}), 1.70-1.61(\mathrm{~m}, 4 \mathrm{H})$, $1.59-1.46(\mathrm{~m}, 5 \mathrm{H}), 1.47-1.34(\mathrm{~m}, 5 \mathrm{H}), 1.30(\mathrm{~d}, J=7.6 \mathrm{~Hz}, 3 \mathrm{H}), 1.24(\mathrm{~s}, 1 \mathrm{H}), 1.17$ (s, $1 \mathrm{H}), 0.77(\mathrm{~s}, 3 \mathrm{H}) ;{ }^{13} \mathrm{C} \mathrm{NMR}\left(101 \mathrm{MHz}, \mathrm{CDCl}_{3}\right) \delta 181.4,170.8,82.7,73.8,72.8,69.9$, $58.9,57.5,48.1,45.3,42.0,40.2,38.9,37.2,36.2,34.1,33.8,33.2,31.8,28.8,25.5$, 21.6, 18.0, 13.2; IR (KBr): $v=3541,3467,2929,1737,1710,1434,734,694 \mathrm{~cm}^{-1}$; HRMS (ESI, $m / z$ ): $[\mathrm{M}+\mathrm{H}]^{+}$calcd for $\mathrm{C}_{24} \mathrm{H}_{37} \mathrm{O}_{6}, 421.2585$; found, 421.2582 .
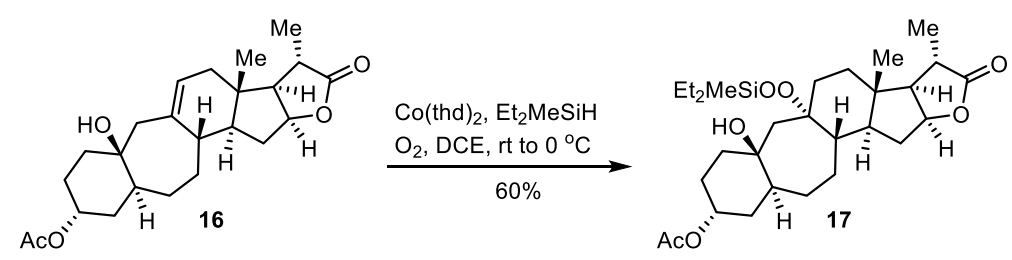

To a solution of 16 (5.2 g, 12.9 mmol, 1.0 equiv) and Co(thd $)_{2}(1.1 \mathrm{~g}, 2.6 \mathrm{mmol}, 0.2$ equiv) in $260 \mathrm{~mL}$ dry DCE was added $\mathrm{Et}_{2} \mathrm{MeSiH}$ (2.8 mL, $19.4 \mathrm{mmol}, 1.5$ equiv) under $\mathrm{O}_{2}$ atmosphere $(1 \mathrm{~atm})$. The reaction mixture was stirred at room temperature for 15 min and then at $0{ }^{\circ} \mathrm{C}$ for $9 \mathrm{~h}$ under $\mathrm{O}_{2}$ (balloon). The reaction mixture was quenched with sat. aq. $\mathrm{NaHCO}_{3}(40 \mathrm{~mL})$ and extracted with $\mathrm{DCM}(3 \times 50 \mathrm{~mL})$. The combined organic layers were washed with brine and dried over $\mathrm{Na}_{2} \mathrm{SO}_{4}$. Removal of the solvent under reduced pressure afforded the crude product which was purified by flash chromatography ( $\mathrm{SiO}_{2}, 7: 1 \rightarrow 6: 1 \rightarrow 5: 1$ petroleum ether:EtOAc) to provide compound $17(4.1 \mathrm{~g}, 60 \%)$ as a white solid.

Compound 17: mp: $170.9-173.8^{\circ} \mathrm{C}$; TLC (petroleum ether:EtOAc, $2: 1 \mathrm{v} / \mathrm{v}$ ): $R_{f}=0.61$; $[\alpha]_{\mathrm{D}}^{25}-11.5\left(c 0.30, \mathrm{CHCl}_{3}\right) ;{ }^{1} \mathrm{H}$ NMR $\left(400 \mathrm{MHz}, \mathrm{CDCl}_{3}\right) \delta 5.03-4.99(\mathrm{~m}, 1 \mathrm{H}), 4.94$ $(\mathrm{td}, J=7.7,4.5 \mathrm{~Hz}, 1 \mathrm{H}), 2.56(\mathrm{q}, J=7.6 \mathrm{~Hz}, 1 \mathrm{H}), 2.28(\mathrm{~d}, J=15.7 \mathrm{~Hz}, 1 \mathrm{H}), 2.25-2.12$ (m, 2H), $2.04(\mathrm{~s}, 3 \mathrm{H}), 2.08-1.95(\mathrm{~m}, 2 \mathrm{H}), 1.85(\mathrm{~d}, J=7.8 \mathrm{~Hz}, 2 \mathrm{H}), 1.76(\mathrm{~d}, J=15.9$ 
$\mathrm{Hz}, 1 \mathrm{H}), 1.79-1.73(\mathrm{~m}, 1 \mathrm{H}), 1.71-1.50(\mathrm{~m}, 6 \mathrm{H}), 1.50-1.34(\mathrm{~m}, 8 \mathrm{H}), 1.31(\mathrm{~d}, J=7.6$ $\mathrm{Hz}, 3 \mathrm{H}), 0.99$ (t, $J=7.9 \mathrm{~Hz}, 6 \mathrm{H}), 0.77$ (s, 3H), 0.70 (q, $J=7.6 \mathrm{~Hz}, 2 \mathrm{H}), 0.68$ (q, $J=7.6$ $\mathrm{Hz}, 2 \mathrm{H}), 0.17$ (s, 3H); ${ }^{13} \mathrm{C} \mathrm{NMR}\left(101 \mathrm{MHz}, \mathrm{CDCl}_{3}\right) \delta 181.5,170.9,84.7,82.7,73.1$, 69.8, 59.1, 53.3, 47.9, 45.4, 42.0, 40.1, 36.9, 36.3, 34.3, 34.2, 33.6, 33.1, 32.1, 27.6, 25.4, 21.7, 18.1, 13.4, 7.0, 5.8, 5.6, -5.0 (one $\mathrm{CH}_{3}$ carbon was overlapped); IR (KBr): $v=3458,2945,2874,1760,1734,1243,826,804 \mathrm{~cm}^{-1}$; HRMS $(\mathrm{ESI}, \mathrm{m} / z):[\mathrm{M}+\mathrm{Na}]^{+}$ calcd for $\mathrm{C}_{29} \mathrm{H}_{48} \mathrm{O}_{7} \mathrm{SiNa}$, 559.3062; found, 559.3061.
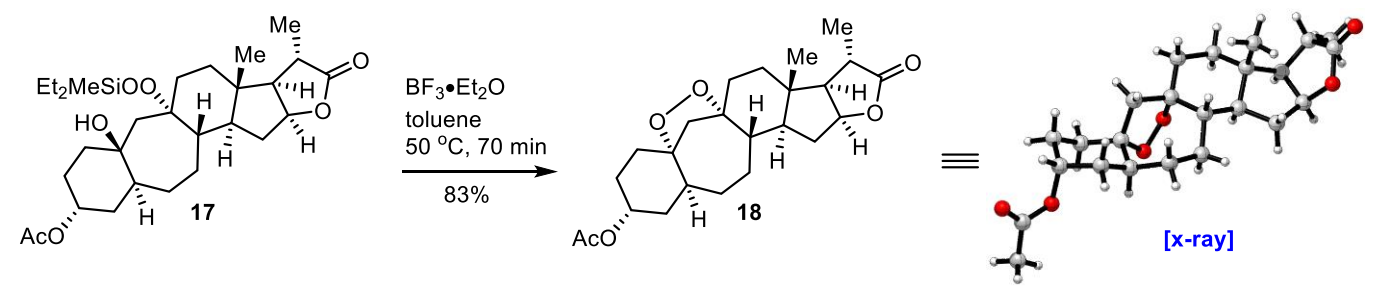

To a solution of 17 (0.8 g, $1.45 \mathrm{mmol}, 1.0$ equiv) in $29 \mathrm{~mL}$ dry toluene was added $\mathrm{BF}_{3} \cdot \mathrm{Et}_{2} \mathrm{O}\left(54 \mu \mathrm{L}, 0.44 \mathrm{mmol}, 0.3\right.$ equiv). The reaction mixture was stirred at $50{ }^{\circ} \mathrm{C}$ for 70 min and quenched with sat. aq. $\mathrm{NaHCO}_{3}(8 \mathrm{~mL})$. The aqueous layer was extracted with EtOAc $(3 \times 15 \mathrm{~mL})$. The combined organic layers were washed with brine, dried over $\mathrm{Na}_{2} \mathrm{SO}_{4}$ and concentrated in vacuo. Removal of the solvent under reduced pressure afforded the crude product which was purified by flash chromatography $\left(\mathrm{SiO}_{2}\right.$, $10: 1 \rightarrow 8: 1$ petroleum ether:acetone) to provide compound $\mathbf{1 8}(505.0 \mathrm{mg}, 83 \%)$ as a white solid.

The single crystal of compound $\mathbf{1 8}$ suitable for X-ray determination was obtained by slow evaporation of a solution of $\mathbf{1 8}$ in petroleum ether and EtOAc at room temperature. Compound 18: mp: $222.4-224.0^{\circ} \mathrm{C}$; TLC (petroleum ether:EtOAc, $2: 1 \mathrm{v} / \mathrm{v}$ ): $R_{f}=0.53$; $[\alpha]_{\mathrm{D}}^{25}+25.6\left(c 1.00, \mathrm{CHCl}_{3}\right) ;{ }^{1} \mathrm{H} \mathrm{NMR}\left(400 \mathrm{MHz}, \mathrm{CDCl}_{3}\right) \delta 4.99-4.91(\mathrm{~m}, 2 \mathrm{H}), 2.57$ $(\mathrm{d}, J=11.8 \mathrm{~Hz}, 1 \mathrm{H}), 2.55(\mathrm{q}, J=7.7 \mathrm{~Hz}, 1 \mathrm{H}), 2.30(\mathrm{dt}, J=13.6,7.2 \mathrm{~Hz}, 1 \mathrm{H}), 2.06-$ $1.97(\mathrm{~m}, 4 \mathrm{H}), 2.03(\mathrm{~s}, 3 \mathrm{H}), 1.97-1.90(\mathrm{~m}, 3 \mathrm{H}), 1.90-1.81(\mathrm{~m}, 3 \mathrm{H}), 1.75-1.67(\mathrm{~m}$, 1H), $1.66-1.60(\mathrm{~m}, 1 \mathrm{H}), 1.59-1.46(\mathrm{~m}, 4 \mathrm{H}), 1.43-1.35(\mathrm{~m}, 1 \mathrm{H}), 1.33-1.26(\mathrm{~m}$, $1 \mathrm{H}), 1.30(\mathrm{~d}, J=7.6 \mathrm{~Hz}, 3 \mathrm{H}), 1.10(\mathrm{td}, J=12.6,3.4 \mathrm{~Hz}, 2 \mathrm{H}), 0.77(\mathrm{~s}, 3 \mathrm{H}) ;{ }^{13} \mathrm{C} \mathrm{NMR}$ 
$\left(101 \mathrm{MHz}, \mathrm{CDCl}_{3}\right) \delta 181.3,170.7,87.8,86.0,82.6,68.1,58.8,50.1,48.5,45.7,41.6$, $37.6,36.3,35.7,35.5,33.3,33.2,32.4,31.8,28.6,27.6,21.5,18.0,13.0 ; \mathrm{IR}(\mathrm{KBr}): v=$ 2937, 2863, 2769, 1733, 1254, 1237, 1183, $1021 \mathrm{~cm}^{-1}$; HRMS (ESI, $\left.m / z\right):[\mathrm{M}+\mathrm{H}]^{+}$calcd for $\mathrm{C}_{24} \mathrm{H}_{35} \mathrm{O}_{6}, 419.2428$; found, 419.2423 .

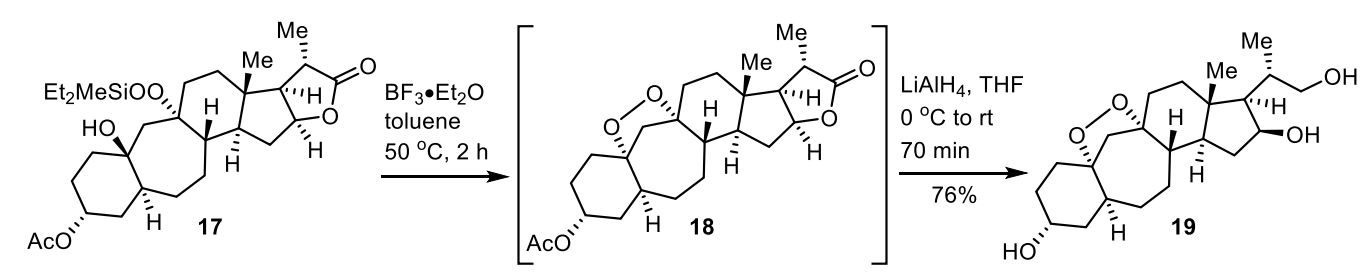

To a solution of 17 (4.6 g, $8.7 \mathrm{mmol}, 1.0$ equiv) in $218 \mathrm{~mL}$ dry toluene was added $\mathrm{BF}_{3} \cdot \mathrm{Et}_{2} \mathrm{O}(0.32 \mathrm{~mL}, 2.6 \mathrm{mmol}, 0.3$ equiv $)$. After stirred at $50{ }^{\circ} \mathrm{C}$ for $1 \mathrm{~h}$, another portion of $\mathrm{BF}_{3} \cdot \mathrm{Et}_{2} \mathrm{O}(0.11 \mathrm{~mL}, 0.9 \mathrm{mmol}, 0.1$ equiv) was added and stirring was continued for another $1 \mathrm{~h}$. The reaction mixture was quenched with solid $\mathrm{NaHCO}_{3}(366.0 \mathrm{mg}, 4.4$ mmol, 0.5 equiv) and stirred at room temperature for $10 \mathrm{~min}$. Removal of the solvent under reduced pressure afforded the crude 18, which was dissolved in $435 \mathrm{~mL}$ dry THF. $\mathrm{LiAlH}_{4}\left(1.7 \mathrm{~g}, 43.5 \mathrm{mmol}, 5.0\right.$ equiv) was added in $5 \mathrm{~min}$ at $0{ }^{\circ} \mathrm{C}$. After stirred at room temperature for another $65 \mathrm{~min}$, the reaction mixture was quenched slowly with $1.7 \mathrm{~mL}$ $\mathrm{H}_{2} \mathrm{O}, 1.7 \mathrm{~mL} 15 \%$ aq. $\mathrm{NaOH}$ and $5.1 \mathrm{~mL} \mathrm{H}_{2} \mathrm{O}$. The mixture was then filtered through celite and the filtrate was concentrated under reduced pressure. The crude residue was purified by flash chromatography $\left(\mathrm{SiO}_{2}, 30: 1 \rightarrow 20: 1 \mathrm{DCM}: \mathrm{MeOH}\right)$ to provide compound $19(2.5 \mathrm{~g}, 76 \%)$ as a white solid.

Compound 19: mp: $234.2-236.4{ }^{\circ} \mathrm{C}$; TLC (petroleum ether:acetone, $2: 1 \mathrm{v} / \mathrm{v}$ ): $R_{f}=$ 0.29; $[\alpha]_{\mathrm{D}}^{25}+170.9(c 0.07, \mathrm{MeOH}) ;{ }^{1} \mathrm{H} \mathrm{NMR}\left(400 \mathrm{MHz}, \mathrm{CD}_{3} \mathrm{OD}\right) \delta 4.37$ (td, $J=7.7$, $4.7 \mathrm{~Hz}, 1 \mathrm{H}), 3.96-3.91(\mathrm{~m}, 1 \mathrm{H}), 3.64(\mathrm{dd}, J=10.5,5.8 \mathrm{~Hz}, 1 \mathrm{H}), 3.48(\mathrm{dd}, J=10.5$, $4.8 \mathrm{~Hz}, 1 \mathrm{H}), 2.71(\mathrm{~d}, J=12.1 \mathrm{~Hz}, 1 \mathrm{H}), 2.25(\mathrm{dt}, J=13.1,7.6 \mathrm{~Hz}, 1 \mathrm{H}), 2.18-2.04(\mathrm{~m}$, $3 \mathrm{H}), 1.99(\mathrm{~d}, J=12.1 \mathrm{~Hz}, 1 \mathrm{H}), 1.94-1.75(\mathrm{~m}, 6 \mathrm{H}), 1.66-1.40(\mathrm{~m}, 6 \mathrm{H}), 1.29-1.16$ (m, 4H), $1.16-1.09(\mathrm{~m}, 1 \mathrm{H}), 1.01$ (d, $J=6.9 \mathrm{~Hz}, 3 \mathrm{H}), 0.97(\mathrm{~s}, 3 \mathrm{H}) ;{ }^{13} \mathrm{C}$ NMR $(101$ $\left.\mathrm{MHz}, \mathrm{CD}_{3} \mathrm{OD}\right) \delta 88.0,85.9,71.2,67.8,64.3,59.6,49.5,45.5,42.1,39.8,37.2,36.0$, $35.1,32.5,32.2,31.5,30.5,27.1,15.6,11.1$; (In $\mathrm{CD}_{3} \mathrm{OD}$, two carbon peaks were 
overlapped, so $\mathrm{C}_{5} \mathrm{D}_{5} \mathrm{~N}$ was used instead) ${ }^{13} \mathrm{C} \mathrm{NMR}\left(101 \mathrm{MHz}, \mathrm{C}_{5} \mathrm{D}_{5} \mathrm{~N}\right) \delta 88.1,85.8$, 71.3, 68.8, 64.0, 60.9, 50.1, 48.1, 45.9, 42.6, 41.3, 37.7, 37.0, 35.7, 33.4, 33.3, 32.9, 32.2, 31.9, 27.8, 17.0, 12.4; IR (KBr): $v=3735,3354,2969,2926,1559,1507,1364$, $668 \mathrm{~cm}^{-1}$; HRMS (ESI, m/z): $[\mathrm{M}+\mathrm{Na}]^{+}$calcd for $\mathrm{C}_{22} \mathrm{H}_{36} \mathrm{O}_{5} \mathrm{Na}, 403.2455$; found, 403.2462 .
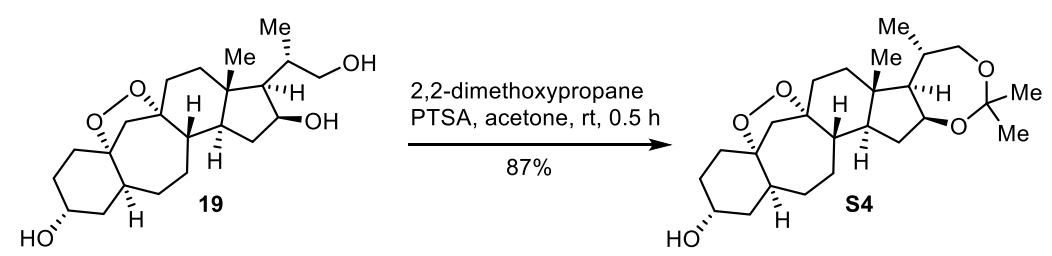

To a stirred solution of 19 (445.0 mg, $1.2 \mathrm{mmol}, 1.0$ equiv) and 2,2-dimethoxypropane (2.6 mL, $21.6 \mathrm{mmol}, 18.0$ equiv) in $90 \mathrm{~mL}$ acetone was added PTSA (56.0 mg, 0.3 mmol, 0.25 equiv). After stirred at room temperature for $0.5 \mathrm{~h}, \mathrm{Et}_{3} \mathrm{~N}(0.32 \mathrm{~mL}, 2.4$ mmol, 2.0 equiv) was added and stirring was continued for another $10 \mathrm{~min}$. Acetone was removed under reduced pressure to afford the crude residue which was purified by flash chromatography ( $\mathrm{SiO}_{2}, 4: 1 \rightarrow 3: 1$ petroleum ether:EtOAc) to furnish compound S4 as a white solid (430.0 mg, 87\%).

Compound S4: mp: $204.7-205.9{ }^{\circ} \mathrm{C}$; TLC (petroleum ether:EtOAc, 2:1 v/v): $R_{f}=$ $0.35 ;[\alpha]_{\mathrm{D}}^{25}+36.0\left(c 1.00, \mathrm{CHCl}_{3}\right) ;{ }^{1} \mathrm{H} \mathrm{NMR}\left(400 \mathrm{MHz}, \mathrm{CDCl}_{3}\right) \delta 4.52$ (q, $J=7.9 \mathrm{~Hz}$, 1H), $4.02-3.98(\mathrm{~m}, 1 \mathrm{H}), 3.80(\mathrm{dd}, J=11.9,5.7 \mathrm{~Hz}, 1 \mathrm{H}), 3.19(\mathrm{t}, J=11.4 \mathrm{~Hz}, 1 \mathrm{H})$, $2.55(\mathrm{~d}, J=11.8 \mathrm{~Hz}, 1 \mathrm{H}), 2.32(\mathrm{tq}, J=12.7,6.4 \mathrm{~Hz}, 1 \mathrm{H}), 2.13(\mathrm{td}, J=13.0,3.8 \mathrm{~Hz}$, 1H), $2.07-2.00(\mathrm{~m}, 1 \mathrm{H}), 1.99-1.80(\mathrm{~m}, 7 \mathrm{H}), 1.71-1.61(\mathrm{~m}, 3 \mathrm{H}), 1.60-1.51(\mathrm{~m}$, 2H), $1.49-1.42(\mathrm{~m}, 2 \mathrm{H}), 1.39(\mathrm{~s}, 3 \mathrm{H}), 1.32-1.18(\mathrm{~m}, 3 \mathrm{H}), 1.27(\mathrm{~s}, 3 \mathrm{H}), 1.11(\mathrm{t}, J=$ $13.2 \mathrm{~Hz}, 2 \mathrm{H}), 0.86(\mathrm{~s}, 3 \mathrm{H}), 0.82(\mathrm{~d}, J=6.6 \mathrm{~Hz}, 3 \mathrm{H}) ;{ }^{13} \mathrm{C} \mathrm{NMR}\left(101 \mathrm{MHz}, \mathrm{CDCl}_{3}\right) \delta$ $100.5,88.0,85.9,71.8,70.0,64.9,58.6,50.1,46.8,45.4,42.2,40.6,37.0,35.1,33.8$, $32.7,32.4,32.1,31.3,31.2,27.6,25.8,23.6,16.8,12.6$; IR $(\mathrm{KBr}): v=3496,2938,2871$, 1374, 1092, 935, 806, $743 \mathrm{~cm}^{-1}$; HRMS (ESI, $\left.m / z\right)$ : $[\mathrm{M}+\mathrm{Na}]^{+}$calcd for $\mathrm{C}_{25} \mathrm{H}_{40} \mathrm{O}_{5} \mathrm{Na}$, 443.2768; found, 443.2768 . 


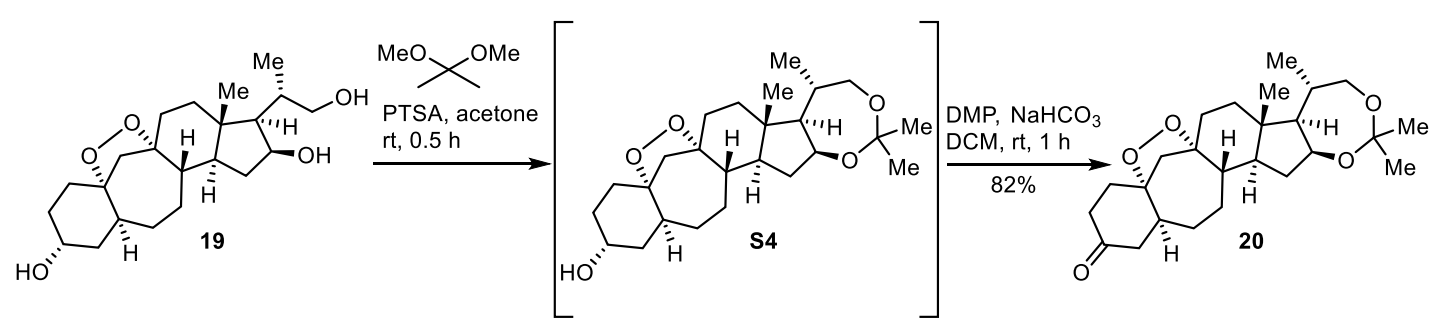

To a stirred solution of $\mathbf{1 9}$ (2.5 g, $6.6 \mathrm{mmol}, 1.0$ equiv) and 2,2-dimethoxypropane (14.5 $\mathrm{mL}, 118.5 \mathrm{mmol}, 18.0$ equiv) in $506 \mathrm{~mL}$ acetone was added PTSA (0.3 g, $1.6 \mathrm{mmol}$, 0.25 equiv). After stirred at room temperature for $0.5 \mathrm{~h}, \mathrm{Et}_{3} \mathrm{~N}(1.8 \mathrm{~mL}, 13.2 \mathrm{mmol}, 2.0$ equiv) was added and stirring was continued for another $10 \mathrm{~min}$. Acetone was removed under reduced pressure and the crude product was dissolved in $165 \mathrm{~mL}$ DCM. Then $\mathrm{NaHCO}_{3}(2.8 \mathrm{~g}, 33.0 \mathrm{mmol}, 5.0$ equiv) and DMP (5.6 g, $13.2 \mathrm{mmol}, 2.0$ equiv) were added successively. After stirred at room temperature for $0.5 \mathrm{~h}$, another portion of DMP ( $1.4 \mathrm{~g}, 3.3 \mathrm{mmol}, 0.5$ equiv) was added and stirring was continued for another $0.5 \mathrm{~h}$ at the same temperature. The reaction mixture was filtered through celite and quenched with sat. aq. $\mathrm{Na}_{2} \mathrm{~S}_{2} \mathrm{O}_{3}(40 \mathrm{~mL})$. The organic layer was separated and the aqueous layer was further extracted with DCM $(2 \times 50 \mathrm{~mL})$. The combined organic layers were washed with brine, dried over $\mathrm{Na}_{2} \mathrm{SO}_{4}$ and concentrated in vacuo. The residue was purified by flash chromatography $\left(\mathrm{SiO}_{2}, 6: 1 \rightarrow 4: 1\right.$ petroleum ether:EtOAc) to furnish compound $\mathbf{2 0}$ as a white solid $(2.3 \mathrm{~g}, 82 \%)$.

Compound 20: mp: $186.3-187.7^{\circ} \mathrm{C}$; TLC (petroleum ether:EtOAc, $2: 1 \mathrm{v} / \mathrm{v}$ ): $R_{f}=0.61$; $[\alpha]_{\mathrm{D}}^{25}+38.6\left(c 0.63, \mathrm{CHCl}_{3}\right) ;{ }^{1} \mathrm{H} \mathrm{NMR}\left(400 \mathrm{MHz}, \mathrm{CDCl}_{3}\right) \delta 4.52(\mathrm{q}, J=7.9 \mathrm{~Hz}, 1 \mathrm{H})$, $3.80(\mathrm{dd}, J=12.0,5.8 \mathrm{~Hz}, 1 \mathrm{H}), 3.19$ (t, $J=11.4 \mathrm{~Hz}, 1 \mathrm{H}), 2.79$ (d, $J=12.0 \mathrm{~Hz}, 1 \mathrm{H})$, $2.46-2.22(\mathrm{~m}, 4 \mathrm{H}), 2.15(\mathrm{~d}, J=12.4 \mathrm{~Hz}, 1 \mathrm{H}), 2.13-2.05(\mathrm{~m}, 3 \mathrm{H}), 2.05-1.93(\mathrm{~m}$, $3 \mathrm{H}), 1.93-1.84(\mathrm{~m}, 1 \mathrm{H}), 1.84-1.73(\mathrm{~m}, 1 \mathrm{H}), 1.73-1.61(\mathrm{~m}, 3 \mathrm{H}), 1.58-1.51(\mathrm{~m}$, 2H), $1.38(\mathrm{~s}, 3 \mathrm{H}), 1.35-1.18(\mathrm{~m}, 4 \mathrm{H}), 1.26(\mathrm{~s}, 3 \mathrm{H}), 0.87(\mathrm{~s}, 3 \mathrm{H}), 0.82(\mathrm{~d}, J=6.5 \mathrm{~Hz}$, $3 \mathrm{H}) ;{ }^{13} \mathrm{C} \mathrm{NMR}\left(101 \mathrm{MHz}, \mathrm{CDCl}_{3}\right) \delta 209.9,100.6,86.3,86.2,71.7,70.0,58.7,51.6$, $47.2,45.9,44.7,42.2,41.6,37.8,37.0,35.7,33.9,32.7,32.6,31.3,27.9,25.9,23.7$, 16.9, 12.6; IR (KBr): $v=2940,2855,1712,1454,1220,1092,1045,895 \mathrm{~cm}^{-1}$; HRMS (ESI, $m / z$ ): $[\mathrm{M}+\mathrm{Na}]^{+}$calcd for $\mathrm{C}_{25} \mathrm{H}_{38} \mathrm{O}_{5} \mathrm{Na}, 441.2611$; found, 441.2613. 


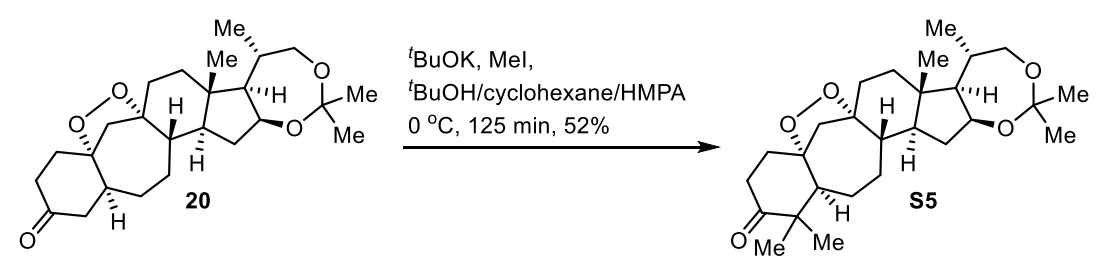

To a solution of 20 (1.1 g, $2.6 \mathrm{mmol}, 1.0$ equiv) in ${ }^{t} \mathrm{BuOH} /$ cyclohexane/HMPA (61.0 $\mathrm{mL}, 2.8: 2.2: 1, \mathrm{v} / \mathrm{v})$ was added ${ }^{t} \mathrm{BuOK}(1.0 \mathrm{M}$ in THF, $7.9 \mathrm{~mL}, 7.9 \mathrm{mmol}, 3.0$ equiv) and MeI (0.49 mL, $7.9 \mathrm{mmol}, 3.0$ equiv) at $0{ }^{\circ} \mathrm{C}$. After stirred at $0{ }^{\circ} \mathrm{C}$ for $25 \mathrm{~min},{ }^{t} \mathrm{BuOK}$ $(5.3 \mathrm{~mL} \times 2,5.3 \mathrm{mmol} \times 2,2.0$ equiv $\times 2)$ and $\mathrm{MeI}(0.33 \mathrm{~mL} \times 2,5.3 \mathrm{mmol} \times 2,2.0$ equiv $\times 2)$ were added in two portions every $25 \mathrm{~min} .{ }^{t} \mathrm{BuOK}(2.6 \mathrm{~mL}, 2.6 \mathrm{mmol}, 1.0$ equiv) and MeI ( $0.16 \mathrm{~mL}, 2.6 \mathrm{mmol}, 1.0$ equiv) were added and stirring was continued for another 25 min under the same condition. Finally, another portion of ${ }^{t} \mathrm{BuOK}(1.3 \mathrm{~mL}, 1.3 \mathrm{mmol}$, 0.5 equiv) and MeI (0.08 mL, $1.3 \mathrm{mmol}, 0.5$ equiv) were added and stirring was continued for $25 \mathrm{~min}$ at $0{ }^{\circ} \mathrm{C}$. The reaction mixture was quenched with water $(20 \mathrm{~mL})$ and extracted with EtOAc $(3 \times 40 \mathrm{~mL})$. The combined organic layers were washed with aq. $\mathrm{NaCl}$ (water/brine $=1: 1, \mathrm{v} / \mathrm{v}, 30 \mathrm{~mL}$ ) for 8 times and dried over $\mathrm{Na}_{2} \mathrm{SO}_{4}$. Removal of the solvent under reduced pressure afforded the crude product which was purified by flash chromatography $\left(\mathrm{SiO}_{2}, 31: 6: 1 \rightarrow 25: 6: 1\right.$ petroleum ether:DCM: EtOAc) to provide compound S5 (610.0 mg, 52\%) as a white solid.

Compound S5: mp: $183.9-185.4{ }^{\circ} \mathrm{C}$; TLC (petroleum ether:EtOAc, $4: 1 \mathrm{v} / \mathrm{v}$ ): $R_{f}=$ $0.57 ;[\alpha]_{\mathrm{D}}^{25}+99.7\left(c 0.31, \mathrm{CHCl}_{3}\right) ;{ }^{1} \mathrm{H} \mathrm{NMR}\left(400 \mathrm{MHz}, \mathrm{CDCl}_{3}\right) \delta 4.53$ (q, $J=7.9 \mathrm{~Hz}$, 1H), $3.80(\mathrm{dd}, J=12.0,5.8 \mathrm{~Hz}, 1 \mathrm{H}), 3.20$ (t, $J=11.4 \mathrm{~Hz}, 1 \mathrm{H}), 2.76(\mathrm{~d}, J=12.2 \mathrm{~Hz}$, 1H), $2.63-2.48(\mathrm{~m}, 1 \mathrm{H}), 2.42-2.26(\mathrm{~m}, 3 \mathrm{H}), 2.08(\mathrm{~d}, J=12.4 \mathrm{~Hz}, 1 \mathrm{H}), 2.06-1.99$ $(\mathrm{m}, 2 \mathrm{H}), 1.98-1.85(\mathrm{~m}, 3 \mathrm{H}), 1.82-1.66(\mathrm{~m}, 3 \mathrm{H}), 1.64-1.48(\mathrm{~m}, 3 \mathrm{H}), 1.39(\mathrm{~s}, 3 \mathrm{H})$, $1.34-1.20(\mathrm{~m}, 4 \mathrm{H}), 1.27(\mathrm{~s}, 3 \mathrm{H}), 1.08(\mathrm{~s}, 3 \mathrm{H}), 0.99(\mathrm{~s}, 3 \mathrm{H}), 0.88(\mathrm{~s}, 3 \mathrm{H}), 0.82(\mathrm{~d}, J=$ $6.6 \mathrm{~Hz}, 3 \mathrm{H}) ;{ }^{13} \mathrm{C} \mathrm{NMR}\left(101 \mathrm{MHz}, \mathrm{CDCl}_{3}\right) \delta 215.8,100.5,86.4,86.2,71.8,70.0,58.7$, 53.7, 48.2, 46.8, 46.5, 44.4, 42.4, 36.9, 35.8, 35.6, 33.7, 31.8, 31.3, 28.4, 25.9, 24.4, 24.2, 23.7, 20.7, 16.8, 12.7; IR (KBr): $v=2944,2870,1709,1454,1224,1092,1075$, $895 \mathrm{~cm}^{-1}$; HRMS (ESI, m/z): $[\mathrm{M}+\mathrm{Na}]^{+}$calcd for $\mathrm{C}_{27} \mathrm{H}_{42} \mathrm{O}_{5} \mathrm{Na}, 469.2924$; found, 
469.2923.
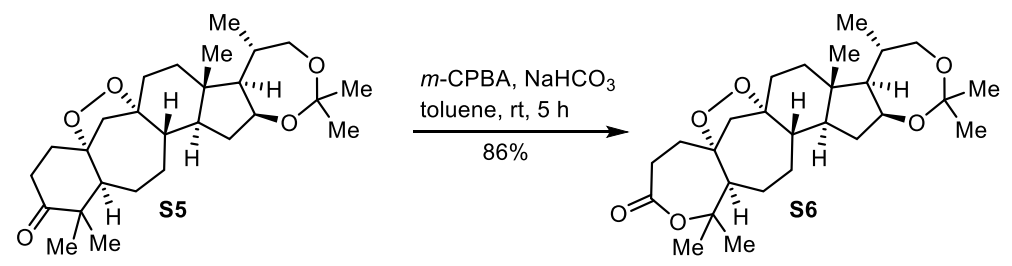

To a stirred solution of $\mathbf{S 5}$ (585.0 mg, $1.3 \mathrm{mmol}, 1.0$ equiv) and $\mathrm{NaHCO}_{3}(687.8 \mathrm{mg}$, $8.2 \mathrm{mmol}, 6.3$ equiv) in $33 \mathrm{~mL}$ toluene was added $85 \% \mathrm{~m}$-CPBA $(800.0 \mathrm{mg}, 3.9 \mathrm{mmol}$, 3.0 equiv) at room temperature. After stirred at the same temperature for $3.5 \mathrm{~h}$, another portion of $85 \% \mathrm{~m}$-CPBA (270.0 $\mathrm{mg}, 1.3 \mathrm{mmol}, 1.0$ equiv) was added and stirring was continued for another $1.5 \mathrm{~h}$ at room temperature. The reaction mixture was quenched with sat. aq. $\mathrm{Na}_{2} \mathrm{~S}_{2} \mathrm{O}_{3}$ and extracted with EtOAc $(3 \times 20 \mathrm{~mL})$. The combined organic layers were washed with brine, dried over $\mathrm{Na}_{2} \mathrm{SO}_{4}$ and concentrated in vacuo. The residue was purified by flash chromatography $\left(\mathrm{SiO}_{2}, 5: 1\right.$ petroleum ether:EtOAc) to furnish compound S6 (520.1 $\mathrm{mg}, 86 \%)$ as a white solid.

Compound S6: mp: $208.9-211.4{ }^{\circ} \mathrm{C}$; TLC (petroleum ether:EtOAc, 4:1 v/v): $R_{f}=$ $0.25 ;[\alpha]_{\mathrm{D}}^{25}+76.2\left(c 0.60, \mathrm{CHCl}_{3}\right) ;{ }^{1} \mathrm{H} \mathrm{NMR}\left(400 \mathrm{MHz}, \mathrm{CDCl}_{3}\right) \delta 4.53(\mathrm{q}, J=7.9 \mathrm{~Hz}$, $1 \mathrm{H}), 3.79(\mathrm{dd}, J=11.9,5.8 \mathrm{~Hz}, 1 \mathrm{H}), 3.18(\mathrm{t}, J=11.4 \mathrm{~Hz}, 1 \mathrm{H}), 2.80-2.56(\mathrm{~m}, 2 \mathrm{H})$, $2.65(\mathrm{~d}, J=12.0 \mathrm{~Hz}, 1 \mathrm{H}), 2.40-2.25(\mathrm{~m}, 1 \mathrm{H}), 2.16-1.93(\mathrm{~m}, 6 \mathrm{H}), 1.97(\mathrm{~d}, J=12.4$ $\mathrm{Hz}, 1 \mathrm{H}), 1.89(\mathrm{t}, J=12.4 \mathrm{~Hz}, 1 \mathrm{H}), 1.73-1.61(\mathrm{~m}, 3 \mathrm{H}), 1.61-1.54(\mathrm{~m}, 1 \mathrm{H}), 1.53-$ $1.44(\mathrm{~m}, 2 \mathrm{H}), 1.413(\mathrm{~s}, 3 \mathrm{H}), 1.405(\mathrm{~s}, 3 \mathrm{H}), 1.38(\mathrm{~s}, 3 \mathrm{H}), 1.32-1.20(\mathrm{~m}, 3 \mathrm{H}), 1.26(\mathrm{~s}$, $3 \mathrm{H}), 0.86(\mathrm{~s}, 3 \mathrm{H}), 0.81(\mathrm{~d}, J=6.5 \mathrm{~Hz}, 3 \mathrm{H}) ;{ }^{13} \mathrm{C} \mathrm{NMR}\left(101 \mathrm{MHz}, \mathrm{CDCl}_{3}\right) \delta 173.8,100.6$, $87.6,86.9,85.8,71.8,70.0,58.7,52.0,48.6,46.6,44.6,42.4,36.8,34.9,33.7,31.9$, $31.7,31.3,31.2,28.8,28.4,25.9,23.7,23.3,16.8,12.7$; IR $(\mathrm{KBr}): v=2948,2871,1719$, 1253, 1223, 1097, 1044, $901 \mathrm{~cm}^{-1}$; HRMS (ESI, $\left.m / z\right)$ : $[\mathrm{M}+\mathrm{Na}]^{+}$calcd for $\mathrm{C}_{27} \mathrm{H}_{42} \mathrm{O}_{6} \mathrm{Na}$, 485.2874; found, 485.2872 . 


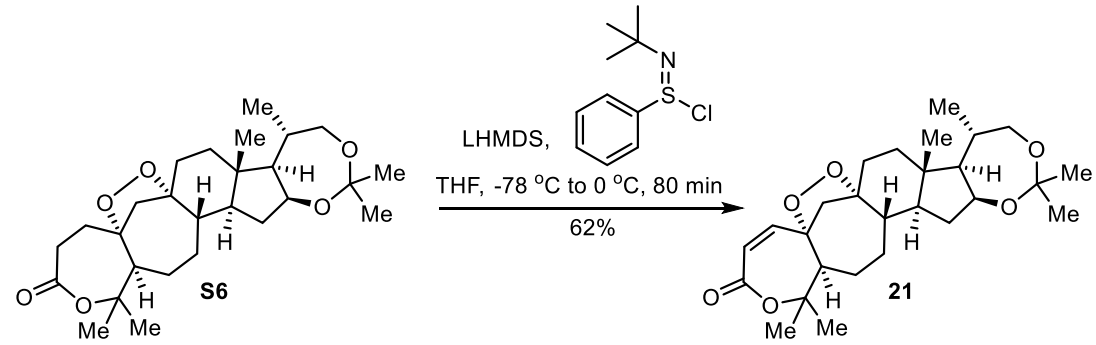

To a stirred solution of $\mathbf{S 6}$ (100.0 $\mathrm{mg}, 0.22 \mathrm{mmol}, 1.0$ equiv) in $5.4 \mathrm{~mL}$ dry THF was added LHMDS (1.0 M solution in THF, $0.3 \mathrm{~mL}, 0.31 \mathrm{mmol}, 1.4$ equiv) at $-78{ }^{\circ} \mathrm{C}$ under argon. After stirring at $-78^{\circ} \mathrm{C}$ for $0.5 \mathrm{~h}$, a solution of $\mathrm{N}$-tert-butylbenzenesulfinimidoyl chloride (64.0 mg, $0.31 \mathrm{mmol}, 1.4$ equiv) in $1.0 \mathrm{~mL}$ dry THF was added dropwise. Stirring was continued for $40 \mathrm{~min}$ at $-78{ }^{\circ} \mathrm{C}$ and $10 \mathrm{~min}$ at $0{ }^{\circ} \mathrm{C}$. The reaction mixture was quenched with sat. aq. $\mathrm{NH}_{4} \mathrm{Cl}$ and extracted with EtOAc $(3 \times 15 \mathrm{~mL})$. The combined organic layers were washed with brine, dried over $\mathrm{Na}_{2} \mathrm{SO}_{4}$ and concentrated in vacuo. The residue was purified by flash chromatography $\left(\mathrm{SiO}_{2}, 19: 1 \rightarrow 16: 1\right.$ petroleum ether:acetone) to furnish compound $21(61.7 \mathrm{mg}, 62 \%)$ as a white solid.

Alternatively, compound $\mathbf{2 1}$ could be prepared in two steps, as shown below:
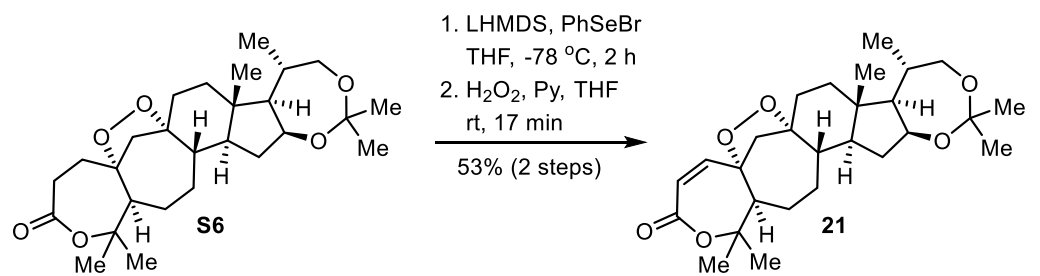

LHMDS (1.3 M solution in THF, $1.6 \mathrm{~mL}, 2.04 \mathrm{mmol}, 2.0$ equiv) was added to a stirred solution of $\mathbf{S 6}$ (475.0 $\mathrm{mg}, 1.02 \mathrm{mmol}, 1.0$ equiv) in $20 \mathrm{~mL}$ dry THF at $-78{ }^{\circ} \mathrm{C}$ under argon. After stirring at $-78{ }^{\circ} \mathrm{C}$ for $0.5 \mathrm{~h}$, a solution of $\mathrm{PhSeBr}(606.5 \mathrm{mg}, 2.57 \mathrm{mmol}$, 2.5 equiv) in $5 \mathrm{~mL}$ dry THF was added. After an additional $1.5 \mathrm{~h}$, the reaction mixture was quenched with sat. aq. $\mathrm{NH}_{4} \mathrm{Cl}$ and extracted with EtOAc $(3 \times 20 \mathrm{~mL})$. The combined organic layers were washed with brine, dried over $\mathrm{Na}_{2} \mathrm{SO}_{4}$ and concentrated in vacuo. The residue was purified by flash column chromatography to afford the desired phenylseleno lactone.

To a solution of the above selenylated lactone in $25 \mathrm{~mL}$ THF was added pyridine $(0.49$ 
mL, 6.12 mmol, 6.0 equiv) and $\mathrm{H}_{2} \mathrm{O}_{2}\left(30 \%\right.$ solution in $\mathrm{H}_{2} \mathrm{O}, 0.21 \mathrm{~mL}, 2.04 \mathrm{mmol}, 2.0$ equiv) at room temperature. After stirring at the same temperature for $5 \mathrm{~min}$, another portion of $\mathrm{H}_{2} \mathrm{O}_{2}$ (30\% solution in $\mathrm{H}_{2} \mathrm{O}, 0.31 \mathrm{~mL}, 3.06 \mathrm{mmol}, 3.0$ equiv) was added and stirring was continued for another $12 \mathrm{~min}$. The reaction mixture was quenched with sat. aq. $\mathrm{Na}_{2} \mathrm{~S}_{2} \mathrm{O}_{3}$ and extracted with EtOAc $(3 \times 20 \mathrm{~mL})$. The combined organic layers were washed with brine, dried over $\mathrm{Na}_{2} \mathrm{SO}_{4}$ and concentrated in vacuo. The residue was purified by flash chromatography $\left(\mathrm{SiO}_{2}, 20: 1 \rightarrow 4: 1\right.$ petroleum ether:acetone) to furnish compound 21 as a white solid (250.7 $\mathrm{mg}, 53 \%)$.

Compound 21: mp: $165.1-166.9^{\circ} \mathrm{C}$; TLC (petroleum ether:EtOAc, $4: 1 \mathrm{v} / \mathrm{v}$ ): $R_{f}=0.23$; $[\alpha]_{\mathrm{D}}^{25}+165.8\left(c 1.00, \mathrm{CHCl}_{3}\right) ;{ }^{1} \mathrm{H} \mathrm{NMR}\left(400 \mathrm{MHz}, \mathrm{CDCl}_{3}\right) \delta 6.24(\mathrm{~d}, J=12.5 \mathrm{~Hz}, 1 \mathrm{H})$, $5.93(\mathrm{~d}, J=12.5 \mathrm{~Hz}, 1 \mathrm{H}), 4.53(\mathrm{q}, J=7.9 \mathrm{~Hz}, 1 \mathrm{H}), 3.80(\mathrm{dd}, J=12.0,5.8 \mathrm{~Hz}, 1 \mathrm{H}), 3.19$ (t, $J=11.4 \mathrm{~Hz}, 1 \mathrm{H}), 2.78(\mathrm{~d}, J=12.3 \mathrm{~Hz}, 1 \mathrm{H}), 2.40(\mathrm{dd}, J=11.7,5.8 \mathrm{~Hz}, 1 \mathrm{H}), 2.36-$ $2.27(\mathrm{~m}, 1 \mathrm{H}), 2.19-2.09(\mathrm{~m}, 1 \mathrm{H}), 2.15(\mathrm{~d}, J=12.5 \mathrm{~Hz}, 1 \mathrm{H}), 2.08-1.95(\mathrm{~m}, 2 \mathrm{H}), 1.87$ $(\mathrm{q}, J=12.7 \mathrm{~Hz}, 1 \mathrm{H}), 1.74-1.66(\mathrm{~m}, 2 \mathrm{H}), 1.66-1.53(\mathrm{~m}, 3 \mathrm{H}), 1.49(\mathrm{td}, J=11.7,4.7$ $\mathrm{Hz}, 1 \mathrm{H}), 1.41(\mathrm{~s}, 3 \mathrm{H}), 1.38(\mathrm{~s}, 6 \mathrm{H}), 1.32-1.18(\mathrm{~m}, 3 \mathrm{H}), 1.26(\mathrm{~s}, 3 \mathrm{H}), 0.86(\mathrm{~s}, 3 \mathrm{H}), 0.81$ $(\mathrm{d}, J=6.5 \mathrm{~Hz}, 3 \mathrm{H}) ;{ }^{13} \mathrm{C} \mathrm{NMR}\left(101 \mathrm{MHz}, \mathrm{CDCl}_{3}\right) \delta 165.4,146.8,119.6,100.6,87.5$, $87.3,82.9,71.7,70.0,58.7,53.1,49.2,46.6,44.5,42.4,36.9,33.6,31.31,31.28,30.2$, 28.8, 28.1, 25.9, 23.7, 21.6, 16.8, 12.7; IR (KBr): $v=2953,2900,1693,1644,1262$, 1016, 985, $809 \mathrm{~cm}^{-1}$; HRMS (ESI, $\left.m / z\right)$ : $[\mathrm{M}+\mathrm{Na}]^{+}$calcd for $\mathrm{C}_{27} \mathrm{H}_{40} \mathrm{O}_{6} \mathrm{Na}, 483.2717$; found, 483.2722 .
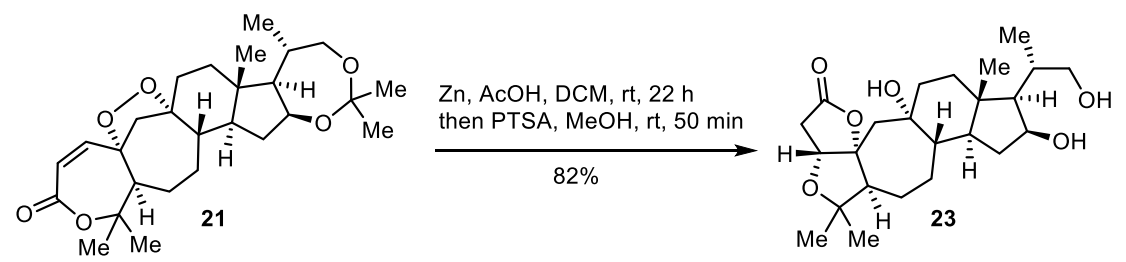

To a solution of 21 (489.0 mg, $1.06 \mathrm{mmol}, 1.0$ equiv) in $22 \mathrm{~mL}$ DCM was added zinc powder (414.1 mg, $6.37 \mathrm{mmol}, 6.0$ equiv) and $\mathrm{AcOH}(0.19 \mathrm{~mL}, 3.19 \mathrm{mmol}, 3.0$ equiv). After stirring at room temperature for $11 \mathrm{~h}$, another portion of $\mathrm{AcOH}(0.19 \mathrm{~mL}, 3.19$ mmol, 3.0 equiv) was added and stirring was continued for $11 \mathrm{~h}$ under the same 
condition. MeOH (21.5 mL) and PTSA (3.03 g, $15.93 \mathrm{mmol}, 15.0$ equiv) were added. After stirred at room temperature for $50 \mathrm{~min}$, the reaction was quenched with $\mathrm{Et}_{3} \mathrm{~N}$ (6 $\mathrm{mL})$. After stirred at room temperature for another $10 \mathrm{~min}$, solvent was removed under reduced pressure. Sat. aq. $\mathrm{NH}_{4} \mathrm{Cl}(20 \mathrm{~mL})$ was added and the aqueous layer was extracted with EtOAc $(3 \times 20 \mathrm{~mL})$. The combined organic layers were washed with brine and dried over $\mathrm{Na}_{2} \mathrm{SO}_{4}$. Removal of the solvent under reduced pressure afforded the crude product which was purified by flash chromatography $\left(\mathrm{SiO}_{2}, 5: 1 \rightarrow 2: 1\right.$ petroleum ether: EtOAc) to provide compound $23(367.9 \mathrm{mg}, 82 \%)$ as a white solid.

Compound 23: mp: $230.1-231.5{ }^{\circ} \mathrm{C}$; TLC (petroleum ether:acetone, $3: 1 \mathrm{v} / \mathrm{v}$ ): $R_{f}=$ $0.21 ;[\alpha]_{\mathrm{D}}^{25}+43.0\left(c 0.62, \mathrm{CHCl}_{3}\right) ;{ }^{1} \mathrm{H} \mathrm{NMR}\left(400 \mathrm{MHz}, \mathrm{CDCl}_{3}\right) \delta 4.40(\mathrm{q}, J=7.6 \mathrm{~Hz}$, $1 \mathrm{H}), 4.15(\mathrm{~d}, J=4.2 \mathrm{~Hz}, 1 \mathrm{H}), 3.74-3.48(\mathrm{~m}, 3 \mathrm{H}), 2.94(\mathrm{~s}, 1 \mathrm{H}), 2.75-2.47(\mathrm{~m}, 3 \mathrm{H})$, $2.35(\mathrm{dd}, J=13.3,4.0 \mathrm{~Hz}, 1 \mathrm{H}), 2.26-2.13(\mathrm{~m}, 2 \mathrm{H}), 1.91(\mathrm{~d}, J=15.1 \mathrm{~Hz}, 1 \mathrm{H}), 1.80(\mathrm{~d}$, $J=15.1 \mathrm{~Hz}, 1 \mathrm{H}), 1.76-1.67(\mathrm{~m}, 3 \mathrm{H}), 1.66-1.54(\mathrm{~m}, 3 \mathrm{H}), 1.46-1.34(\mathrm{~m}, 3 \mathrm{H}), 1.31$ (s, 3H), $1.27-1.15(\mathrm{~m}, 2 \mathrm{H}), 1.10(\mathrm{~s}, 3 \mathrm{H}), 0.95(\mathrm{~d}, J=6.9 \mathrm{~Hz}, 3 \mathrm{H}), 0.93(\mathrm{~s}, 3 \mathrm{H}) ;{ }^{13} \mathrm{C}$ NMR $\left(101 \mathrm{MHz}, \mathrm{CDCl}_{3}\right) \delta 174.1,100.0,85.0,81.0,72.8,72.5,71.0,62.2,59.4,49.5$, $46.2,45.7,42.9,38.9,35.8,35.6,35.4,32.5,28.9,27.9,26.4,22.4,16.9,12.5$; IR (KBr): $v=3579,3392,2932,2862,1777,1263,911,736 \mathrm{~cm}^{-1}$; HRMS $($ ESI, $m / z):[\mathrm{M}+\mathrm{Na}]^{+}$ calcd for $\mathrm{C}_{24} \mathrm{H}_{38} \mathrm{O}_{6} \mathrm{Na}, 445.2561$; found, 445.2566.
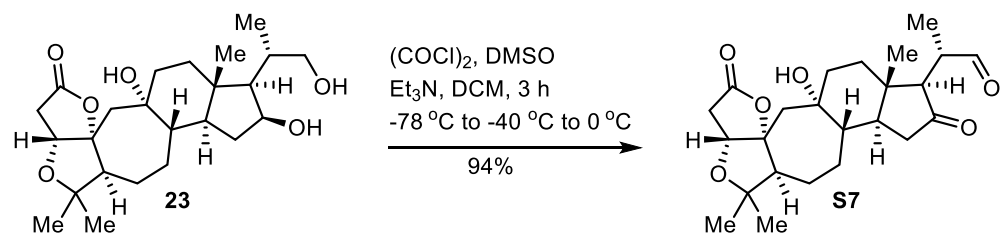

To a solution of $(\mathrm{COCl})_{2}(361 \mu \mathrm{L}, 4.25 \mathrm{mmol}, 5.0$ equiv $)$ in $22 \mathrm{~mL}$ dry DCM was added $\operatorname{DMSO}\left(454 \mu \mathrm{L}, 6.38 \mathrm{mmol}, 7.5\right.$ equiv) at $-78^{\circ} \mathrm{C}$. After stirring at the same temperature for $45 \mathrm{~min}$, a solution of 23 (360.0 $\mathrm{mg}, 0.85 \mathrm{mmol}, 1.0$ equiv) in $28 \mathrm{~mL}$ DCM was added and stirring was continued for $35 \mathrm{~min}$ at $-78{ }^{\circ} \mathrm{C}$. The reaction mixture was warmed to $-40{ }^{\circ} \mathrm{C}$ and stirring was continued for another $45 \mathrm{~min}$ at the same temperature. $\mathrm{Et}_{3} \mathrm{~N}(1.42 \mathrm{~mL}, 10.20 \mathrm{mmol}, 12.0$ equiv) was added and stirring was 
continued for $50 \mathrm{~min}$ at $-40{ }^{\circ} \mathrm{C}$ and finally $5 \mathrm{~min}$ at $0{ }^{\circ} \mathrm{C}$. The reaction mixture was quenched with sat. aq. $\mathrm{NH}_{4} \mathrm{Cl}$ and extracted with $\mathrm{DCM}(3 \times 20 \mathrm{~mL})$. The combined organic layers were washed with brine, dried over $\mathrm{Na}_{2} \mathrm{SO}_{4}$ and concentrated in vacuo. The residue was purified by flash chromatography $\left(\mathrm{SiO}_{2}, 6: 1 \rightarrow 5: 1\right.$ petroleum ether:acetone) to furnish compound $\mathbf{S} 7(335.2 \mathrm{mg}, 94 \%)$ as a white foam.

Compound S7: TLC (petroleum ether:acetone, 3:1 v/v): $R_{f}=0.32 ;[\alpha]_{\mathrm{D}}^{25}-58.6(c 1.00$, $\left.\mathrm{CHCl}_{3}\right) ;{ }^{1} \mathrm{H} \mathrm{NMR}\left(400 \mathrm{MHz}, \mathrm{CDCl}_{3}\right) \delta 9.79(\mathrm{~d}, J=2.1 \mathrm{~Hz}, 1 \mathrm{H}), 4.17(\mathrm{dd}, J=4.2,1.5$ $\mathrm{Hz}, 1 \mathrm{H}), 3.17(\mathrm{~s}, 1 \mathrm{H}), 2.74-2.66(\mathrm{~m}, 2 \mathrm{H}), 2.56-2.49(\mathrm{~m}, 2 \mathrm{H}), 2.45-2.33(\mathrm{~m}, 2 \mathrm{H})$, $2.24(\mathrm{dd}, J=18.3,7.9 \mathrm{~Hz}, 1 \mathrm{H}), 2.02-1.91(\mathrm{~m}, 1 \mathrm{H}), 1.95(\mathrm{~d}, J=15.2 \mathrm{~Hz}, 1 \mathrm{H}), 1.82(\mathrm{~d}$, $J=15.2 \mathrm{~Hz}, 1 \mathrm{H}), 1.82-1.73(\mathrm{~m}, 2 \mathrm{H}), 1.73-1.63(\mathrm{~m}, 2 \mathrm{H}), 1.56-1.41(\mathrm{~m}, 3 \mathrm{H}), 1.32$ $(\mathrm{s}, 3 \mathrm{H}), 1.31-1.22(\mathrm{~m}, 1 \mathrm{H}), 1.115(\mathrm{~d}, J=6.8 \mathrm{~Hz}, 3 \mathrm{H}), 1.11(\mathrm{~s}, 3 \mathrm{H}), 0.82(\mathrm{~s}, 3 \mathrm{H}) ;{ }^{13} \mathrm{C}$ NMR (101 MHz, $\left.\mathrm{CDCl}_{3}\right) \delta 217.1,203.5,173.9,99.6,85.0,81.0,72.4,65.1,59.4,48.8$, $45.7,43.7,43.5,42.3,38.4,37.7,35.7,33.8,28.8,27.7,26.7,22.4,13.3,13.0 ; \mathrm{IR}(\mathrm{KBr})$ : $v=3574,2971,2933,1779,1734,1188,911,734 \mathrm{~cm}^{-1}$; HRMS $($ ESI, $m / z):[\mathrm{M}+\mathrm{Na}]^{+}$ calcd for $\mathrm{C}_{24} \mathrm{H}_{34} \mathrm{O}_{6} \mathrm{Na}, 441.2248$; found, 441.2249 .

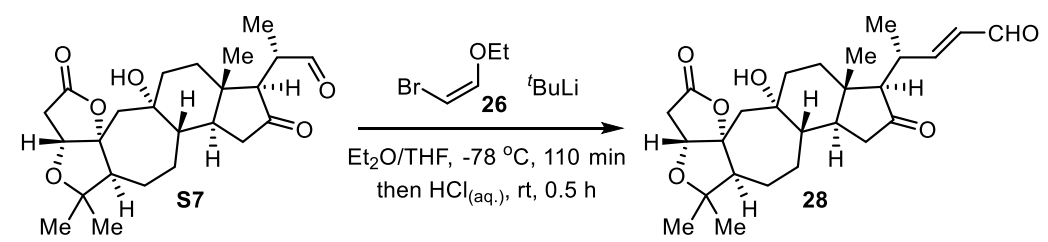

To a solution of (Z)-1-bromo-2-ethoxyethylene (26) (157.4 mg, $1.05 \mathrm{mmol}, 3.5$ equiv) in $7.2 \mathrm{~mL}$ dry $\mathrm{Et}_{2} \mathrm{O}$ was added ${ }^{t} \mathrm{BuLi}(1.3 \mathrm{M}$ in pentane, $1.62 \mathrm{~mL}, 2.10 \mathrm{mmol}, 7.0$ equiv) dropwise. After the reaction was stirred at $-78^{\circ} \mathrm{C}$ for $50 \mathrm{~min}$, a solution of aldehyde $\mathbf{S} 7$ (125.0 mg, $0.30 \mathrm{mmol}, 1.0$ equiv) in $4.2 \mathrm{~mL} \mathrm{Et}_{2} \mathrm{O}$ and $3.3 \mathrm{~mL}$ THF was added. The reaction mixture was stirred at $-78^{\circ} \mathrm{C}$ for another $1 \mathrm{~h}$ before being quenched with $5 \mathrm{~mL}$ $4 \%$ aq. $\mathrm{HCl}$ and $5 \mathrm{~mL} \mathrm{H}_{2} \mathrm{O}$. The resulting mixture was stirred at room temperature for $0.5 \mathrm{~h}$ and quenched with sat. aq. $\mathrm{NaHCO}_{3}$. The aqueous layer was extracted with EtOAc $(3 \times 15 \mathrm{~mL})$ and the combined organic layers were washed with brine, dried over $\mathrm{Na}_{2} \mathrm{SO}_{4}$ and concentrated in vacuo. The residue was purified by flash chromatography $\left(\mathrm{SiO}_{2}\right.$, 
$6: 1 \rightarrow 5: 1$ petroleum ether:acetone) to furnish compound $28(78.6 \mathrm{mg}, 59 \%)$ as a colorless oil.

Compound 28: TLC (petroleum ether:acetone, $3: 1 \mathrm{v} / \mathrm{v}): R_{f}=0.32 ;[\alpha]_{\mathrm{D}}^{25}-29.9(c 0.35$, $\left.\mathrm{CHCl}_{3}\right) ;{ }^{1} \mathrm{H} \mathrm{NMR}\left(400 \mathrm{MHz}, \mathrm{CDCl}_{3}\right) \delta 9.50(\mathrm{~d}, J=7.9 \mathrm{~Hz}, 1 \mathrm{H}), 7.18(\mathrm{dd}, J=15.7,8.0$ Hz, 1H), 6.07 (dd, $J=15.7,7.9 \mathrm{~Hz}, 1 \mathrm{H}), 4.17(\mathrm{dd}, J=4.3,1.5 \mathrm{~Hz}, 1 \mathrm{H}), 3.18(\mathrm{~s}, 1 \mathrm{H})$, $2.80-2.64(\mathrm{~m}, 3 \mathrm{H}), 2.37(\mathrm{dd}, J=13.3,4.4 \mathrm{~Hz}, 1 \mathrm{H}), 2.29-2.18(\mathrm{~m}, 2 \mathrm{H}), 2.11(\mathrm{~d}, J=$ $6.5 \mathrm{~Hz}, 1 \mathrm{H}), 1.95(\mathrm{~d}, J=15.2 \mathrm{~Hz}, 1 \mathrm{H}), 1.91-1.74(\mathrm{~m}, 3 \mathrm{H}), 1.82(\mathrm{~d}, J=15.2 \mathrm{~Hz}, 1 \mathrm{H})$, $1.72-1.64(\mathrm{~m}, 2 \mathrm{H}), 1.57-1.40(\mathrm{~m}, 5 \mathrm{H}), 1.32(\mathrm{~s}, 3 \mathrm{H}), 1.22(\mathrm{~d}, J=7.0 \mathrm{~Hz}, 3 \mathrm{H}), 1.11$ (s, 3H), 0.79 (s, 3H); ${ }^{13} \mathrm{C}$ NMR (101 MHz, $\left.\mathrm{CDCl}_{3}\right) \delta 216.6,194.6,173.8,163.4,131.6$, 99.6, 85.0, 81.0, 72.5, 68.3, 59.4, 48.7, 45.7, 43.3, 43.0, 38.7, 38.4, 35.7, 34.7, 33.7, 28.8, 27.7, 26.6, 22.3, 20.0, 13.6; IR (KBr): $v=3574,2929,2853,1779,1732,1682$, 1631, $734 \mathrm{~cm}^{-1}$; HRMS (ESI, $\mathrm{m} / z$ ): $[\mathrm{M}+\mathrm{H}]^{+}$calcd for $\mathrm{C}_{26} \mathrm{H}_{37} \mathrm{O}_{6}, 445.2585$; found, 445.2579.
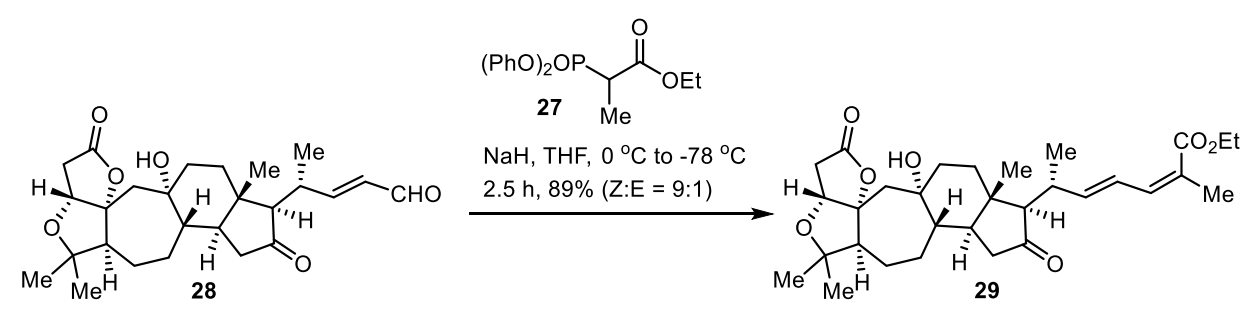

To a suspension of sodium hydride (60\% dispersion in mineral oil, $29.6 \mathrm{mg}, 0.74 \mathrm{mmol}$, 2.8 equiv) in $2.6 \mathrm{~mL}$ dry THF was added dropwise phosphonate 27 (216.3 mg, 0.65 mmol, 2.5 equiv) at $0{ }^{\circ} \mathrm{C}$. After stirred for $0.5 \mathrm{~h}$ at the same temperature, the reaction mixture was cooled to $-78^{\circ} \mathrm{C}$ and a solution of enal 28 (115.0 $\mathrm{mg}, 0.26 \mathrm{mmol}, 1.0$ equiv) in $4.0 \mathrm{~mL}$ THF was added. The reaction mixture was stirred for $2 \mathrm{~h}$ at $-78{ }^{\circ} \mathrm{C}$ before quenched with sat. aq. $\mathrm{NH}_{4} \mathrm{Cl}$. The reaction mixture was extracted with EtOAc $(3 \times 10$ $\mathrm{mL}$ ). The combined organic layers were washed with brine, dried over $\mathrm{Na}_{2} \mathrm{SO}_{4}$ and concentrated in vacuo. The residue was purified by flash chromatography $\left(\mathrm{SiO}_{2}\right.$, $10: 1 \rightarrow 8: 1$ petroleum ether: acetone) to furnish compound $29(122.2 \mathrm{mg}, 89 \%, Z: E=$ 9:1) as a white foam. 
Compound 29: TLC (petroleum ether:acetone, $3: 1 \mathrm{v} / \mathrm{v}): R_{f}=0.45 ;[\alpha]_{\mathrm{D}}^{25}-23.9(c 1.00$, $\left.\mathrm{CHCl}_{3}\right) ;{ }^{1} \mathrm{H} \mathrm{NMR}\left(400 \mathrm{MHz}, \mathrm{CDCl}_{3}\right) \delta 7.09(\mathrm{dd}, J=15.3,11.1 \mathrm{~Hz}, 1 \mathrm{H}), 6.40(\mathrm{~d}, J=$ $11.1 \mathrm{~Hz}, 1 \mathrm{H}), 6.21(\mathrm{dd}, J=15.3,8.1 \mathrm{~Hz}, 1 \mathrm{H}), 4.20(\mathrm{q}, J=7.2 \mathrm{~Hz}, 2 \mathrm{H}), 4.16(\mathrm{~d}, J=4.0$ $\mathrm{Hz}, 1 \mathrm{H}), 3.10(\mathrm{~s}, 1 \mathrm{H}), 2.76-2.54(\mathrm{~m}, 3 \mathrm{H}), 2.36(\mathrm{dd}, J=13.3,4.3 \mathrm{~Hz}, 1 \mathrm{H}), 2.22-2.11$ (m, 2H), 2.02 (d, $J=5.3 \mathrm{~Hz}, 1 \mathrm{H}), 1.93$ (d, $J=16.0 \mathrm{~Hz}, 1 \mathrm{H}), 1.92(\mathrm{~s}, 3 \mathrm{H}), 1.86-1.71$ (m, 3H), $1.82(\mathrm{~d}, J=16.0 \mathrm{~Hz}, 1 \mathrm{H}), 1.70-1.59(\mathrm{~m}, 3 \mathrm{H}), 1.58-1.46(\mathrm{~m}, 3 \mathrm{H}), 1.45-$ $1.36(\mathrm{~m}, 1 \mathrm{H}), 1.31(\mathrm{~s}, 3 \mathrm{H}), 1.30(\mathrm{t}, J=7.2 \mathrm{~Hz}, 3 \mathrm{H}), 1.18(\mathrm{~d}, J=7.0 \mathrm{~Hz}, 3 \mathrm{H}), 1.10(\mathrm{~s}$, 3H), 0.77 (s, 3H); ${ }^{13} \mathrm{C}$ NMR (101 MHz, $\left.\mathrm{CDCl}_{3}\right) \delta 217.1,173.9,167.9,146.1,141.3$, 126.6, 124.2, 99.6, 85.0, 81.0, 72.6, 68.8, 60.2, 59.4, 48.6, 45.8, 43.2, 43.0, 39.0, 38.4, $35.7,34.7,33.6,28.8,27.7,26.7,22.4,21.1,20.8,14.5,13.9$; IR $(\mathrm{KBr}): v=3576,2974$, 2932, 1782, 1734, 1700, 1634, $733 \mathrm{~cm}^{-1}$; $\mathrm{HRMS}$ (ESI, $\left.m / z\right)$ : $[\mathrm{M}+\mathrm{H}]^{+}$calcd for $\mathrm{C}_{31} \mathrm{H}_{45} \mathrm{O}_{7}$, 529.3160; found, 529.3156 .
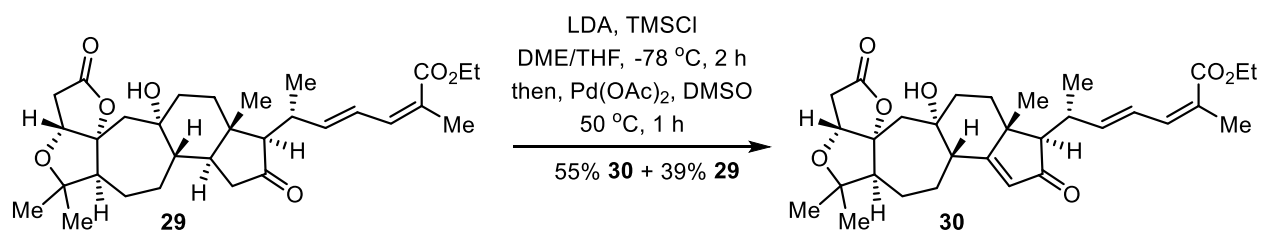

To a solution of ester 29 (90.0 mg, $0.17 \mathrm{mmol}, 1.0$ equiv) in $2.4 \mathrm{~mL}$ DME was added dropwise freshly prepared LDA (1.0 M in THF, $0.51 \mathrm{~mL}, 0.51 \mathrm{mmol}, 3.0$ equiv) at -78 ${ }^{\circ} \mathrm{C}$. After stirred at $-78{ }^{\circ} \mathrm{C}$ for $0.5 \mathrm{~h}, 0.3 \mathrm{~mL}$ dry THF was added (to avoid freezing of the reaction mixture) and stirring was continued for another $0.5 \mathrm{~h}$. TMSCl $(89 \mu \mathrm{L}, 0.68$ mmol, 4.0 equiv) was added and the reaction mixture was stirred for $1 \mathrm{~h}$ at $-78^{\circ} \mathrm{C}$. The reaction mixture was quenched with sat. aq. $\mathrm{NaHCO}_{3}$ and extracted with $\mathrm{EtOAc}(3 \times 10$ $\mathrm{mL}$ ). The combined organic layers were washed with brine, dried over $\mathrm{Na}_{2} \mathrm{SO}_{4}$ and concentrated in vacuo. The residue was used directly in the next step.

A solution of the crude silyl enol ether obtained above and $\mathrm{Pd}(\mathrm{OAc})_{2}(38.2 \mathrm{mg}, 0.17$ mmol, 1.0 equiv) in $3.0 \mathrm{~mL}$ DMSO was stirred at $50{ }^{\circ} \mathrm{C}$ for $1 \mathrm{~h}$ before quenched with sat. aq. $\mathrm{NH}_{4} \mathrm{Cl}(5 \mathrm{~mL})$. The reaction mixture was extracted with EtOAc $(3 \times 10 \mathrm{~mL})$. The combined organic layers were washed with brine, dried over $\mathrm{Na}_{2} \mathrm{SO}_{4}$ and concentrated 
in vacuo. The residue was purified by flash chromatography $\left(\mathrm{SiO}_{2}, 8: 1 \rightarrow 6: 1 \rightarrow 5: 1\right.$ petroleum ether:acetone) to furnish compound $\mathbf{3 0}(49.2 \mathrm{mg}, 55 \%)$ as a white foam and recovered $29(35.1 \mathrm{mg}, 39 \%)$.

Compound 30: TLC (petroleum ether:acetone, 3:1 v/v): $R_{f}=0.29 ;[\alpha]_{\mathrm{D}}^{25}+124.5(c 0.47$, $\left.\mathrm{CHCl}_{3}\right) ;{ }^{1} \mathrm{H} \mathrm{NMR}\left(400 \mathrm{MHz}, \mathrm{CDCl}_{3}\right) \delta 7.13(\mathrm{dd}, J=15.3,11.1 \mathrm{~Hz}, 1 \mathrm{H}), 6.42(\mathrm{~d}, J=$ $11.1 \mathrm{~Hz}, 1 \mathrm{H}), 6.15(\mathrm{dd}, J=15.3,8.1 \mathrm{~Hz}, 1 \mathrm{H}), 5.80(\mathrm{~s}, 1 \mathrm{H}), 4.21$ (q, $J=7.1 \mathrm{~Hz}, 2 \mathrm{H})$, $4.18(\mathrm{~d}, J=2.2 \mathrm{~Hz}, 1 \mathrm{H}), 3.33(\mathrm{~s}, 1 \mathrm{H}), 2.88-2.78(\mathrm{~m}, 1 \mathrm{H}), 2.74-2.64(\mathrm{~m}, 2 \mathrm{H}), 2.39$ $(\mathrm{d}, J=13.6 \mathrm{~Hz}, 1 \mathrm{H}), 2.37(\mathrm{~d}, J=12.4 \mathrm{~Hz}, 1 \mathrm{H}), 2.28(\mathrm{~d}, J=4.3 \mathrm{~Hz}, 1 \mathrm{H}), 2.07$ (d, $J=$ $15.2 \mathrm{~Hz}, 1 \mathrm{H}), 2.03-1.96(\mathrm{~m}, 1 \mathrm{H}), 1.93(\mathrm{~s}, 3 \mathrm{H}), 1.91-1.74(\mathrm{~m}, 5 \mathrm{H}), 1.88$ (d, $J=15.2$ $\mathrm{Hz}, 1 \mathrm{H}), 1.58-1.52(\mathrm{~m}, 1 \mathrm{H}), 1.34(\mathrm{~s}, 3 \mathrm{H}), 1.31$ (t, $J=7.2 \mathrm{~Hz}, 3 \mathrm{H}), 1.29$ (d, J=7.2 Hz, 3H), 1.19 (s, 3H), 1.12 (s, 3H); ${ }^{13} \mathrm{C}$ NMR (101 MHz, $\left.\mathrm{CDCl}_{3}\right) \delta$ 207.8, 186.2, 173.6, $167.9,145.4,141.1,127.1,126.9,124.3,98.9,84.7,80.9,75.2,64.7,60.3,60.0,50.1$, $47.2,45.2,38.0,35.8,35.5,35.3,28.5,26.8,25.3,22.0,21.8,21.3,20.8,14.5 ; \operatorname{IR}(\mathrm{KBr})$ : $v=3570,2927,2868,1781,1693,1633,1614,738 \mathrm{~cm}^{-1}$; HRMS $($ ESI, $m / z):[\mathrm{M}+\mathrm{H}]^{+}$ calcd for $\mathrm{C}_{31} \mathrm{H}_{43} \mathrm{O}_{7}, 527.3003$; found, 527.3008.
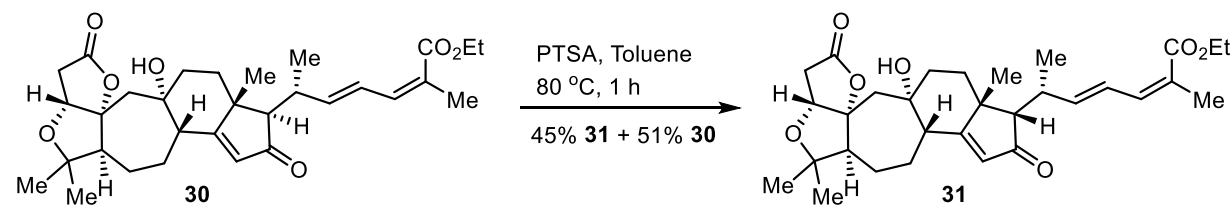

To a solution of $\mathbf{3 0}(60.0 \mathrm{mg}, 0.11 \mathrm{mmol}, 1.0$ equiv) in $5.7 \mathrm{~mL}$ toluene was added PTSA (4.3 mg, $0.023 \mathrm{mmol}, 0.2$ equiv). After the reaction mixture was stirred at $80{ }^{\circ} \mathrm{C}$ for 1 $\mathrm{h}$, the reaction mixture was quenched with sat. aq. $\mathrm{NaHCO}_{3}(4 \mathrm{~mL})$ and extracted with EtOAc $(3 \times 10 \mathrm{~mL})$. The combined organic layers were washed with brine, dried over $\mathrm{Na}_{2} \mathrm{SO}_{4}$ and concentrated in vacuo. The residue was purified by flash chromatography $\left(\mathrm{SiO}_{2}, 30: 1 \rightarrow 25: 1\right.$ DCM:acetone) to furnish compound $\mathbf{3 1}(27.0 \mathrm{mg}, 45 \%)$ as a yellow foam and recovered $\mathbf{3 0}(30.6 \mathrm{mg}, 51 \%)$.

Compound 31: TLC (DCM:acetone, 8:1 v/v): $R_{f}=0.64 ;[\alpha]_{\mathrm{D}}^{25}+153.6\left(c 0.41, \mathrm{CHCl}_{3}\right)$; ${ }^{1} \mathrm{H}$ NMR $\left(400 \mathrm{MHz}, \mathrm{CDCl}_{3}\right) \delta 7.10(\mathrm{dd}, J=15.3,11.1 \mathrm{~Hz}, 1 \mathrm{H}), 6.43(\mathrm{~d}, J=11.2 \mathrm{~Hz}$, $1 \mathrm{H}), 6.13(\mathrm{dd}, J=15.3,7.9 \mathrm{~Hz}, 1 \mathrm{H}), 5.79(\mathrm{~s}, 1 \mathrm{H}), 4.21(\mathrm{q}, J=7.1 \mathrm{~Hz}, 2 \mathrm{H}), 4.18(\mathrm{~d}, J=$ 
$4.7 \mathrm{~Hz}, 1 \mathrm{H}), 3.33(\mathrm{~s}, 1 \mathrm{H}), 2.84-2.74(\mathrm{~m}, 1 \mathrm{H}), 2.73-2.64(\mathrm{~m}, 2 \mathrm{H}), 2.43-2.34(\mathrm{~m}$, 2H), $2.23(\mathrm{~d}, J=4.6 \mathrm{~Hz}, 1 \mathrm{H}), 2.15(\mathrm{td}, J=13.1,4.3 \mathrm{~Hz}, 1 \mathrm{H}), 2.07(\mathrm{~d}, J=15.2 \mathrm{~Hz}, 1 \mathrm{H})$, $2.03-1.96(\mathrm{~m}, 1 \mathrm{H}), 1.94(\mathrm{~s}, 3 \mathrm{H}), 1.92-1.78(\mathrm{~m}, 3 \mathrm{H}), 1.89$ (d, $J=15.2 \mathrm{~Hz}, 1 \mathrm{H}), 1.65$ $-1.60(\mathrm{~m}, 1 \mathrm{H}), 1.51(\mathrm{dt}, J=12.9,2.6 \mathrm{~Hz}, 1 \mathrm{H}), 1.34(\mathrm{~s}, 3 \mathrm{H}), 1.31(\mathrm{t}, J=7.1 \mathrm{~Hz}, 3 \mathrm{H})$, $1.26(\mathrm{~s}, 3 \mathrm{H}), 1.13(\mathrm{~d}, J=6.8 \mathrm{~Hz}, 3 \mathrm{H}), 1.12(\mathrm{~s}, 3 \mathrm{H}) ;{ }^{13} \mathrm{C} \mathrm{NMR}\left(101 \mathrm{MHz}, \mathrm{CDCl}_{3}\right) \delta$ 209.1, 187.3, 173.5, 167.9, 146.2, 141.0, 127.1, 126.1, 124.7, 98.9, 84.7, 80.9, 75.5, 62.7, 60.3, 60.1, 50.4, 46.1, 45.2, 38.2, 36.2, 35.3, 29.1, 28.4, 27.3, 26.8, 25.4, 21.9, 20.8, 17.6, 14.5; IR (KBr): $v=3570,2926,1782,1693,1614,1455,808,736 \mathrm{~cm}^{-1}$; HRMS (ESI, $m / z$ ): $[\mathrm{M}+\mathrm{H}]^{+}$calcd for $\mathrm{C}_{31} \mathrm{H}_{43} \mathrm{O}_{7}, 527.3003$; found, 527.3004.

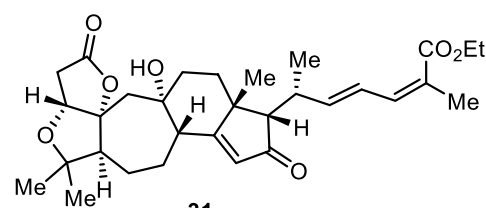

31
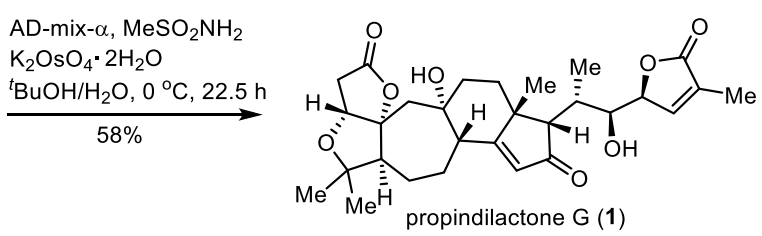

A solution of AD-mix- $\alpha$ (41.0 mg, $0.03 \mathrm{mmol}, 1.0$ equiv), $\mathrm{MeSO}_{2} \mathrm{NH}_{2}(2.9 \mathrm{mg}, 0.03$ mmol, 1.0 equiv) and $\mathrm{K}_{2} \mathrm{OsO}_{4} \cdot 2 \mathrm{H}_{2} \mathrm{O}\left(0.3 \mathrm{mg}, 0.9 \mu \mathrm{mol}, 0.03\right.$ equiv) in ${ }^{t} \mathrm{BuOH}$ and water $(2.4 \mathrm{~mL}, 1: 1, \mathrm{v} / \mathrm{v})$ was stirred at room temperature for $10 \mathrm{~min}$ and $0{ }^{\circ} \mathrm{C}$ for $10 \mathrm{~min}$. The yellow reaction mixture was transferred into a reaction tube containing 31 (16.0 $\mathrm{mg}$, $0.03 \mathrm{mmol}, 1.0$ equiv) at $0{ }^{\circ} \mathrm{C}$. The reaction mixture was stirred at $0{ }^{\circ} \mathrm{C}$ for $22.5 \mathrm{~h}$ and quenched with sat. aq. $\mathrm{Na}_{2} \mathrm{~S}_{2} \mathrm{O}_{3}$. The aqueous layer was extracted with EtOAc $(3 \times 15$ $\mathrm{mL}$ ) and the combined organic layers were washed with brine, dried over $\mathrm{Na}_{2} \mathrm{SO}_{4}$ and concentrated in vacuo. The residue was purified by flash chromatography $\left(\mathrm{SiO}_{2}\right.$, $60: 1 \rightarrow 50: 1 \mathrm{DCM}: \mathrm{MeOH})$ to furnish propindilactone $\mathrm{G}(\mathbf{1})(9.1 \mathrm{mg}, 58 \%)$ as a white solid.

Propindilactone G (1): $\mathrm{mp}: 241.4-242.5^{\circ} \mathrm{C}$; TLC (DCM:acetone, $\left.6: 1 \mathrm{v} / \mathrm{v}\right): R_{f}=0.14$; $[\alpha]_{\mathrm{D}}^{25}+59.6(c 0.15, \mathrm{MeOH}) ;{ }^{1} \mathrm{H} \mathrm{NMR}\left(400 \mathrm{MHz}, \mathrm{CDCl}_{3}\right) \delta 7.11(\mathrm{~s}, 1 \mathrm{H}), 5.83(\mathrm{~s}, 1 \mathrm{H})$, $4.99(\mathrm{q}, J=2.2 \mathrm{~Hz}, 1 \mathrm{H}), 4.24(\mathrm{~d}, J=5.5 \mathrm{~Hz}, 1 \mathrm{H}), 4.19(\mathrm{~d}, J=4.2 \mathrm{~Hz}, 1 \mathrm{H}), 4.00(\mathrm{dd}, J$ $=6.4,4.8 \mathrm{~Hz}, 1 \mathrm{H}), 3.42(\mathrm{~s}, 1 \mathrm{H}), 2.85(\mathrm{~s}, 1 \mathrm{H}), 2.80-2.64(\mathrm{~m}, 2 \mathrm{H}), 2.44(\mathrm{~d}, J=11.5 \mathrm{~Hz}$, 1H), 2.37 (dd, $J=13.4,4.5 \mathrm{~Hz}, 1 \mathrm{H}), 2.23-2.14(\mathrm{~m}, 2 \mathrm{H}), 2.10(\mathrm{~d}, J=15.1 \mathrm{~Hz}, 1 \mathrm{H})$, 
$2.06-1.98(\mathrm{~m}, 1 \mathrm{H}), 1.95(\mathrm{~s}, 3 \mathrm{H}), 1.94-1.77(\mathrm{~m}, 4 \mathrm{H}), 1.70-1.62(\mathrm{~m}, 1 \mathrm{H}), 1.47-1.35$ $(\mathrm{m}, 2 \mathrm{H}), 1.35$ (s, 3H), 1.29 (s, 3H), $1.13(\mathrm{~s}, 3 \mathrm{H}), 0.99$ (d, $J=7.0 \mathrm{~Hz}, 3 \mathrm{H}) ;{ }^{13} \mathrm{C} \mathrm{NMR}$ $\left(101 \mathrm{MHz}, \mathrm{CDCl}_{3}\right) \delta 211.7,191.3,174.6,173.6,147.3,130.7,126.6,98.8,84.7,83.0$, $80.9,75.8,74.0,60.1,57.5,50.7,45.7,45.0,38.1,35.2,34.3,28.37,28.39,27.3,26.7$, 25.3, 21.9, 15.5, 11.0; ${ }^{1} \mathrm{H}$ NMR (400 MHz, $\left.\mathrm{C}_{5} \mathrm{D}_{5} \mathrm{~N}\right) \delta 7.12$ (brs, $\left.1 \mathrm{H}\right), 6.83$ (d, $J=7.5$ $\mathrm{Hz}, 1 \mathrm{H}), 6.08(\mathrm{~d}, J=1.5 \mathrm{~Hz}, 1 \mathrm{H}), 5.26(\mathrm{~s}, 1 \mathrm{H}), 5.06(\mathrm{~s}, 1 \mathrm{H}), 4.72(\mathrm{t}, J=7.5 \mathrm{~Hz}, 1 \mathrm{H})$, $4.25(\mathrm{~d}, J=4.9 \mathrm{~Hz}, 1 \mathrm{H}), 3.40(\mathrm{~d}, J=1.6 \mathrm{~Hz}, 1 \mathrm{H}), 3.01(\mathrm{dd}, J=18.0,5.1 \mathrm{~Hz}, 1 \mathrm{H}), 2.76$ $(\mathrm{d}, J=18.0 \mathrm{~Hz}, 1 \mathrm{H}), 2.64-2.55(\mathrm{~m}, 1 \mathrm{H}), 2.53-2.47(\mathrm{~m}, 1 \mathrm{H}), 2.46(\mathrm{dd}, J=13.5,4.0$ $\mathrm{Hz}, 1 \mathrm{H}), 2.46-2.39(\mathrm{~m}, 1 \mathrm{H}), 2.22(\mathrm{ABd}, J=15.3 \mathrm{~Hz}, 1 \mathrm{H}), 2.08(\mathrm{ABd}, J=15.4 \mathrm{~Hz}$, 1H), $1.98-1.92(\mathrm{~m}, 1 \mathrm{H}), 1.94-1.85(\mathrm{~m}, 1 \mathrm{H}), 1.90-1.83(\mathrm{~m}, 1 \mathrm{H}), 1.83(\mathrm{~s}, 3 \mathrm{H}), 1.69$ $-1.62(\mathrm{~m}, 1 \mathrm{H}), 1.63-1.58(\mathrm{~m}, 1 \mathrm{H}), 1.39-1.31(\mathrm{~m}, 1 \mathrm{H}), 1.29(\mathrm{~s}, 3 \mathrm{H}), 1.26(\mathrm{~d}, J=6.8$ $\mathrm{Hz}, 3 \mathrm{H}), 1.25(\mathrm{~s}, 3 \mathrm{H}), 1.22-1.16(\mathrm{~m}, 1 \mathrm{H}), 1.09$ (s, 3H); ${ }^{13} \mathrm{C} \mathrm{NMR}\left(151 \mathrm{MHz}, \mathrm{C}_{5} \mathrm{D}_{5} \mathrm{~N}\right)$ $\delta 211.0,191.0,175.2,175.2,149.3,130.1,127.3,99.3,84.7,82.3,81.8,75.6,72.1,60.1$, $57.7,50.2,45.7,45.6,38.1,36.6,36.1,29.1,28.3,28.0,26.8,26.6,22.5,14.1,10.9$. IR (KBr): $v=3563,3457,2971,2927,1749,1667,1608,734 \mathrm{~cm}^{-1}$; HRMS (ESI, $\left.m / z\right)$ : $[\mathrm{M}+\mathrm{H}]^{+}$calcd for $\mathrm{C}_{29} \mathrm{H}_{39} \mathrm{O}_{8}, 515.2639$; found, 515.2643. 


\section{NMR comparison of synthetic and natural propindilactone $G$}

${ }^{1} \mathrm{H}$ NMR data comparison of synthetic and natural propindilactone $G$

\begin{tabular}{|c|c|c|c|c|}
\hline position & $\begin{array}{c}\text { natural sample } \\
\left(500 \mathrm{MHz} \text { in } \mathrm{C}_{5} \mathrm{D}_{5} \mathrm{~N}\right)\end{array}$ & $\begin{array}{l}\text { our synthetic sample } \\
\qquad \begin{array}{c}(400 \mathrm{MHz} \text { in } \\
\left.\mathrm{C}_{5} \mathrm{D}_{5} \mathrm{~N}\right)\end{array}\end{array}$ & $\Delta \delta$ & $\begin{array}{c}\text { Yang's synthetic } \\
\text { sample }^{3} \\
\left(400 \mathrm{MHz} \text { in } \mathrm{C}_{5} \mathrm{D}_{5} \mathrm{~N}\right)\end{array}$ \\
\hline 1 & $4.25(\mathrm{~d}, 4.5)$ & $4.25(\mathrm{~d}, 4.9)$ & 0 & $4.25(\mathrm{~d}, 5.0)$ \\
\hline $2 \alpha$ & $2.75(\mathrm{~d}, 17.5)$ & $2.76(\mathrm{~d}, 18.0)$ & 0.01 & $2.75(\mathrm{~d}, 18.0)$ \\
\hline $2 \beta$ & $3.01(\mathrm{dd}, 17.5,4.5)$ & $3.01(\mathrm{dd}, 18.0,5.1)$ & 0 & $3.01(\mathrm{dd}, 18.0,5.1)$ \\
\hline 5 & $2.45(\mathrm{dd}, 13.5,4.0)$ & $2.46(\mathrm{dd}, 13.5,4.0)$ & 0.01 & $2.46(\mathrm{dd}, 13.5,3.9)$ \\
\hline $6 \alpha$ & $1.63-1.55(\mathrm{~m})$ & $1.63-1.58(\mathrm{~m})$ & 0.01 & $1.63-1.59(\mathrm{~m})$ \\
\hline $6 \beta$ & $1.34-1.32(\mathrm{~m})$ & $1.39-1.31(\mathrm{~m})$ & 0.02 & $1.38-1.33(\mathrm{~m})$ \\
\hline $7 \alpha$ & $1.92(\mathrm{~m})$ & $1.98-1.92(\mathrm{~m})$ & 0.03 & $1.97-1.92(\mathrm{~m})$ \\
\hline $7 \beta$ & $1.88(\mathrm{~m})$ & $1.94-1.85(\mathrm{~m})$ & 0.02 & $1.92-1.88(\mathrm{~m})$ \\
\hline 8 & $2.51-2.46(\mathrm{~m})$ & $2.53-2.47(\mathrm{~m})$ & 0.01 & $2.52-2.47(\mathrm{~m})$ \\
\hline $11 \alpha$ & $1.91-1.83(\mathrm{~m})$ & $1.90-1.83(\mathrm{~m})$ & 0 & $1.96-1.85(\mathrm{~m})$ \\
\hline $11 \beta$ & $1.66-1.60(\mathrm{~m})$ & $1.69-1.62(\mathrm{~m})$ & 0.02 & $1.69-1.63(\mathrm{~m})$ \\
\hline $12 \alpha$ & $2.34(\mathrm{~m})$ & $2.46-2.39(\mathrm{~m})$ & 0.08 & $2.43-2.34(\mathrm{~m})$ \\
\hline $12 \beta$ & $1.64-1.61(\mathrm{~m})$ & $1.22-1.16(\mathrm{~m})^{\mathrm{a}}$ & - & $1.64-1.61(\mathrm{~m})$ \\
\hline 15 & $6.07(\mathrm{~s})$ & $6.08(\mathrm{~d}, 1.5)$ & 0.01 & $6.08(\mathrm{~d}, 1.1)$ \\
\hline 17 & $3.38(\mathrm{~s})$ & $3.40(\mathrm{~d}, 1.6)$ & 0.02 & $3.39(\mathrm{~d}, 1.3)$ \\
\hline 18 & $1.24(\mathrm{~s})$ & $1.25(\mathrm{~s})$ & 0.01 & $1.24(\mathrm{~s})$ \\
\hline $19 \alpha$ & $2.20(\mathrm{ABd}, 15.5)$ & $2.22(\mathrm{ABd}, 15.3)$ & 0.02 & $2.22(\mathrm{ABd}, 15.3)$ \\
\hline $19 \beta$ & $2.10(\mathrm{ABd}, 15.5)$ & $2.08(\mathrm{ABd}, 15.4)$ & - & $2.08(\mathrm{ABd}, 15.3)$ \\
\hline 20 & $2.60-2.55(\mathrm{~m})$ & $2.64-2.55(\mathrm{~m})$ & 0.02 & $2.63-2.55(\mathrm{~m})$ \\
\hline 21 & $1.25(\mathrm{~d}, 7.5)$ & $1.26(\mathrm{~d}, 6.8)$ & 0.01 & $1.26(\mathrm{~d}, 6.7)$ \\
\hline 22 & $4.72(\mathrm{~d}, 9.0)$ & $4.72(\mathrm{t}, 7.5)^{\mathrm{b}}$ & 0 & $4.71(\mathrm{~d}, 8.3)$ \\
\hline 23 & 5.26 (brs) & $5.26(\mathrm{~s})$ & 0 & $5.26(\mathrm{~d}, 8.3)$ \\
\hline 24 & 7.12 (brs) & 7.12 (brs) & 0 & $7.11-7.12$ \\
\hline 27 & $1.83(\mathrm{~s})$ & $1.83(\mathrm{~s})$ & 0 & $1.83(\mathrm{~s})$ \\
\hline 29 & $1.09(\mathrm{~s})$ & $1.09(\mathrm{~s})$ & 0 & $1.09(\mathrm{~s})$ \\
\hline 30 & $1.28(\mathrm{~s})$ & $1.29(\mathrm{~s})$ & 0.01 & $1.29(\mathrm{~s})$ \\
\hline 9-OH & - & $5.06(\mathrm{~s})$ & & - \\
\hline $22-\mathrm{OH}$ & - & $6.83(\mathrm{~d}, 7.5)$ & & - \\
\hline
\end{tabular}

aThe chemical shift of 12 $\beta-\mathrm{H}$, despite different from literature's reported value, was determined by HSQC spectrum (see page $\mathrm{S} 108$ for details). ${ }^{b}$ When $\mathrm{D}_{2} \mathrm{O}$ was added to the synthetic sample of propindilactone $\mathrm{G}$ in $\mathrm{C}_{5} \mathrm{D}_{5} \mathrm{~N}$, the signal of $\mathrm{C} 22-\mathrm{H}$ changed from triplet $(J=7.5 \mathrm{~Hz})$ to doublet $(J=8.8 \mathrm{~Hz})$. 
${ }^{13} \mathrm{C}$ NMR data comparison of synthetic and natural (+)-propindilactone G

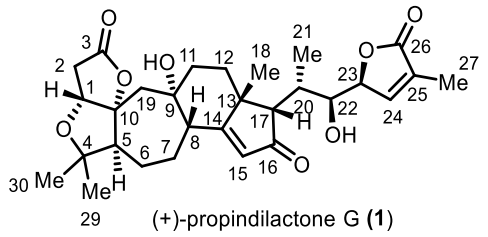

\begin{tabular}{|c|c|c|c|c|}
\hline position & $\begin{array}{c}\text { natural sample } \\
\left(126 \mathrm{MHz} \text { in } \mathrm{C}_{5} \mathrm{D}_{5} \mathrm{~N}\right)\end{array}$ & $\begin{array}{l}\text { our synthetic sample } \\
\left(151 \mathrm{MHz} \text { in } \mathrm{C}_{5} \mathrm{D}_{5} \mathrm{~N}\right)\end{array}$ & $\Delta \delta$ & $\begin{array}{c}\text { Yang's synthetic sample } \\
\left(126 \mathrm{MHz} \text { in } \mathrm{C}_{5} \mathrm{D}_{5} \mathrm{~N}\right)\end{array}$ \\
\hline 1 & 81.8 & 81.8 & 0 & 81.9 \\
\hline 2 & 36.1 & 36.1 & 0 & 36.2 \\
\hline 3 & 175.3 & 175.2 & -0.1 & 175.2 \\
\hline 4 & 84.7 & 84.7 & 0 & 84.8 \\
\hline 5 & 60.1 & 60.1 & 0 & 60.2 \\
\hline 6 & 26.5 & 26.8 & 0.3 & 26.7 \\
\hline 7 & 26.8 & 26.6 & -0.2 & 26.9 \\
\hline 8 & 50.1 & 50.2 & 0.1 & 50.3 \\
\hline 9 & 75.7 & 75.6 & -0.1 & 75.8 \\
\hline 10 & 99.4 & 99.3 & -0.1 & 99.4 \\
\hline 11 & 38.0 & 38.1 & 0.1 & 38.2 \\
\hline 12 & 28.4 & 28.3 & -0.1 & 28.5 \\
\hline 13 & 45.7 & 45.7 & 0 & 45.8 \\
\hline 14 & 191.1 & 191.0 & -0.1 & 191.0 \\
\hline 15 & 127.2 & 127.3 & 0.1 & 127.4 \\
\hline 16 & 211.2 & 211.0 & -0.2 & 211.0 \\
\hline 17 & 57.8 & 57.7 & -0.1 & 57.9 \\
\hline 18 & 27.9 & 28.0 & 0.1 & 28.0 \\
\hline 19 & 45.6 & 45.6 & 0 & 45.8 \\
\hline 20 & 36.5 & 36.6 & 0.1 & 36.6 \\
\hline 21 & 14.1 & 14.1 & 0 & 14.3 \\
\hline 22 & 72.2 & 72.1 & -0.1 & 72.4 \\
\hline 23 & 82.3 & 82.3 & 0 & 82.4 \\
\hline 24 & 149.3 & 149.3 & 0 & 149.3 \\
\hline 25 & 130.1 & 130.1 & 0 & 130.2 \\
\hline 26 & 175.3 & 175.2 & -0.1 & 175.2 \\
\hline 27 & 10.8 & 10.9 & 0.1 & 10.9 \\
\hline 29 & 22.5 & 22.5 & 0 & 22.6 \\
\hline 30 & 29.0 & 29.1 & 0.1 & 29.2 \\
\hline
\end{tabular}


NMR spectra comparison of Yang's and our synthetic propindilactone $\mathrm{G}$ in $\mathrm{CDCl}_{3}$
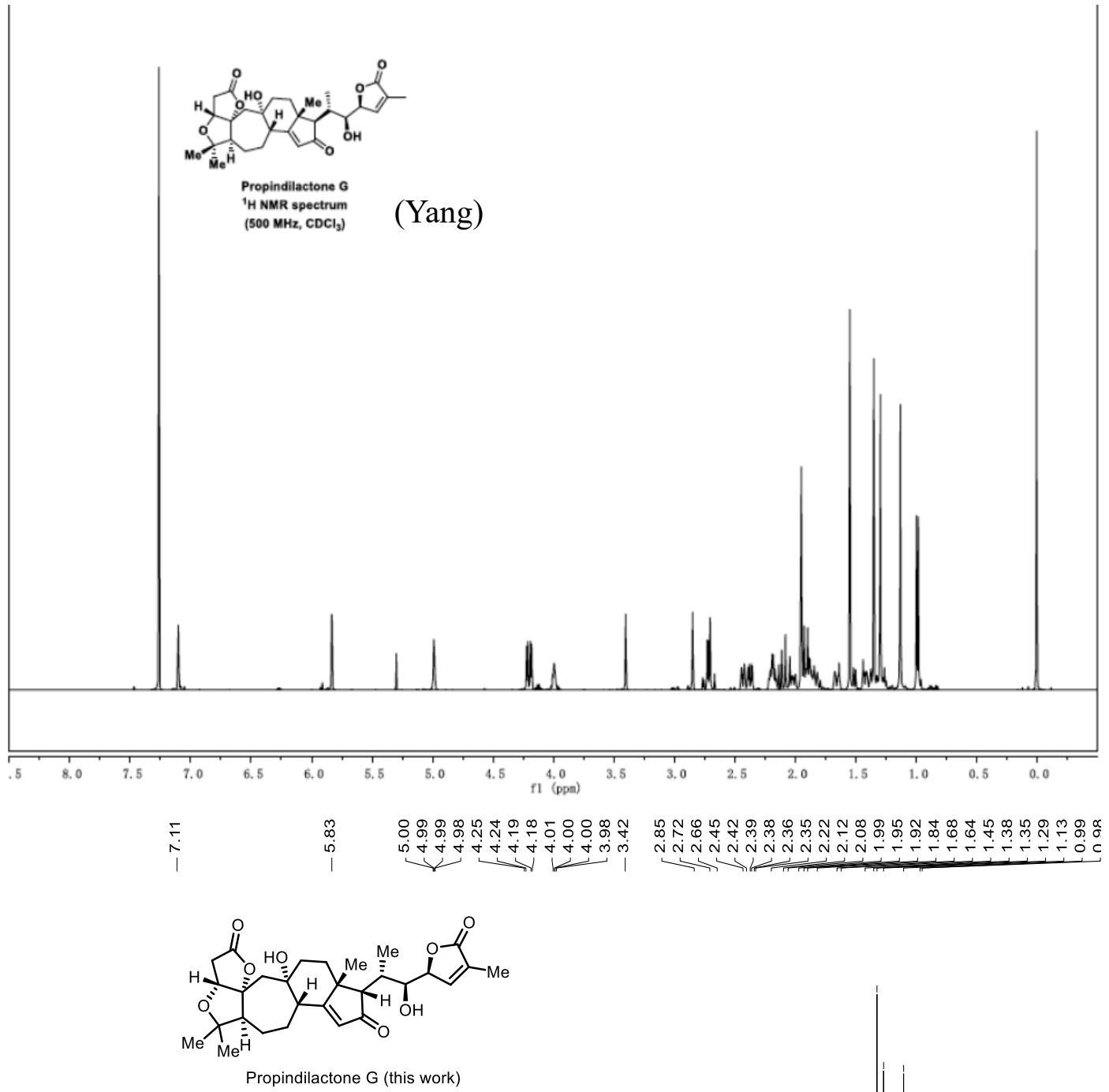

${ }^{1} \mathrm{H} \mathrm{NMR}\left(\mathrm{CDCl}_{3}, 400 \mathrm{MHz}\right)$

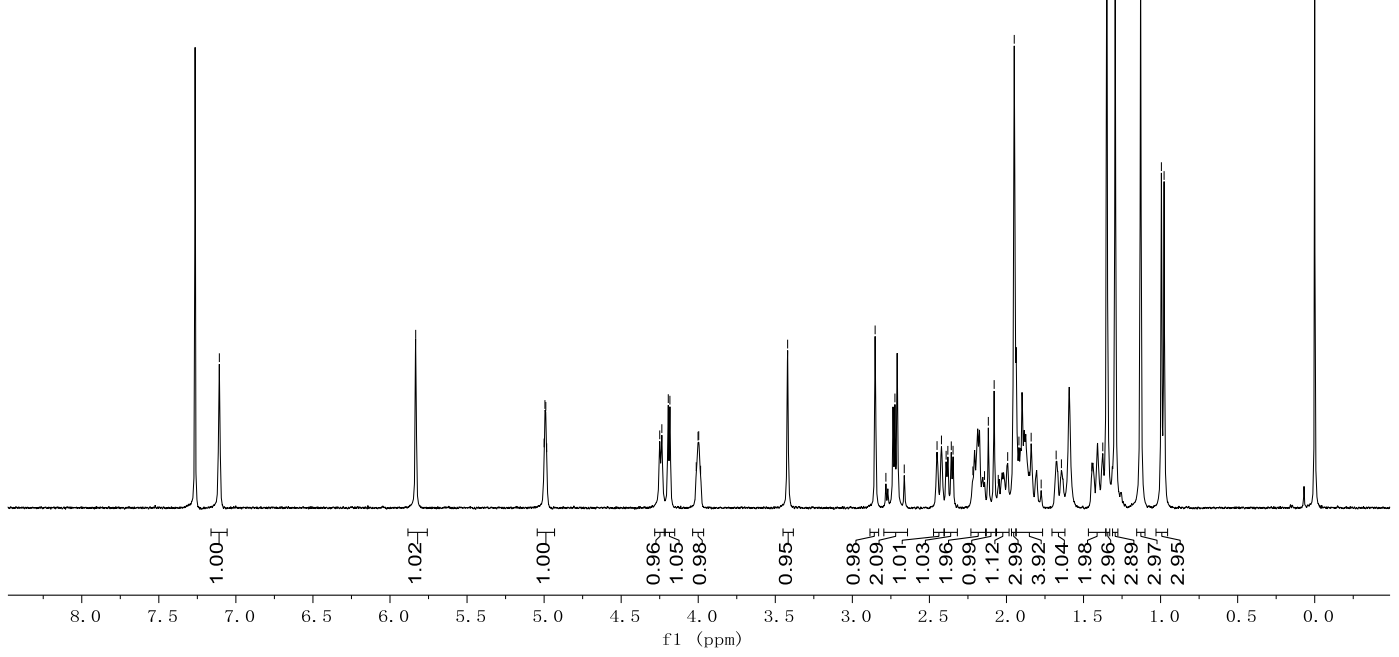




$$
\text { (Yang) }
$$

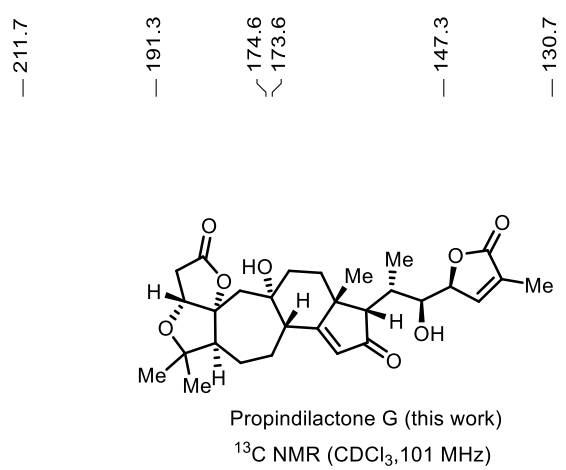

${ }^{13} \mathrm{C} \mathrm{NMR}\left(\mathrm{CDCl}_{3}, 101 \mathrm{MHz}\right)$

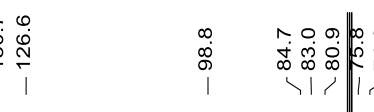

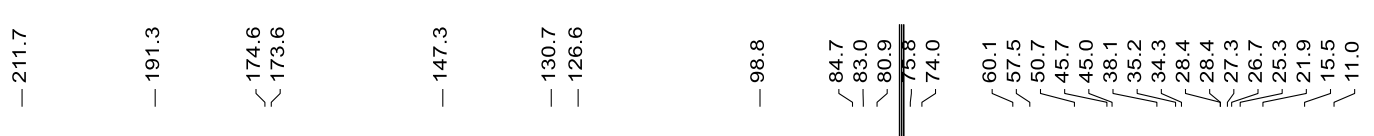

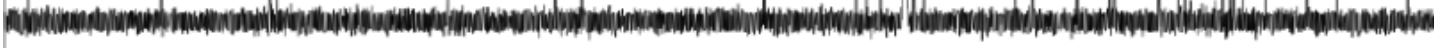
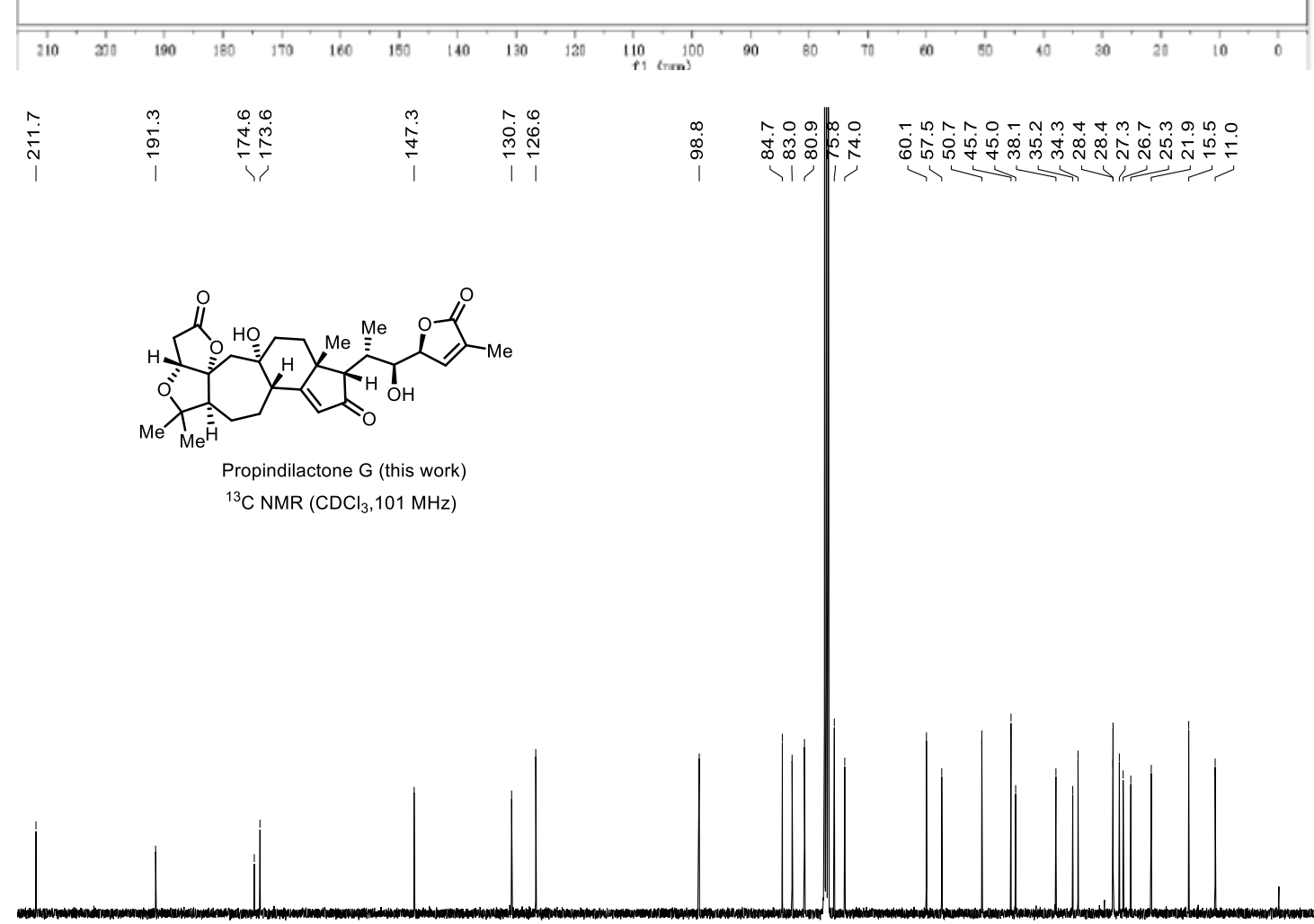

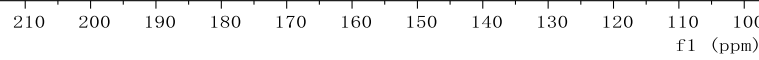


NMR spectra comparison of natural and synthetic propindilactone $G$ in $C_{5} D_{5} N$
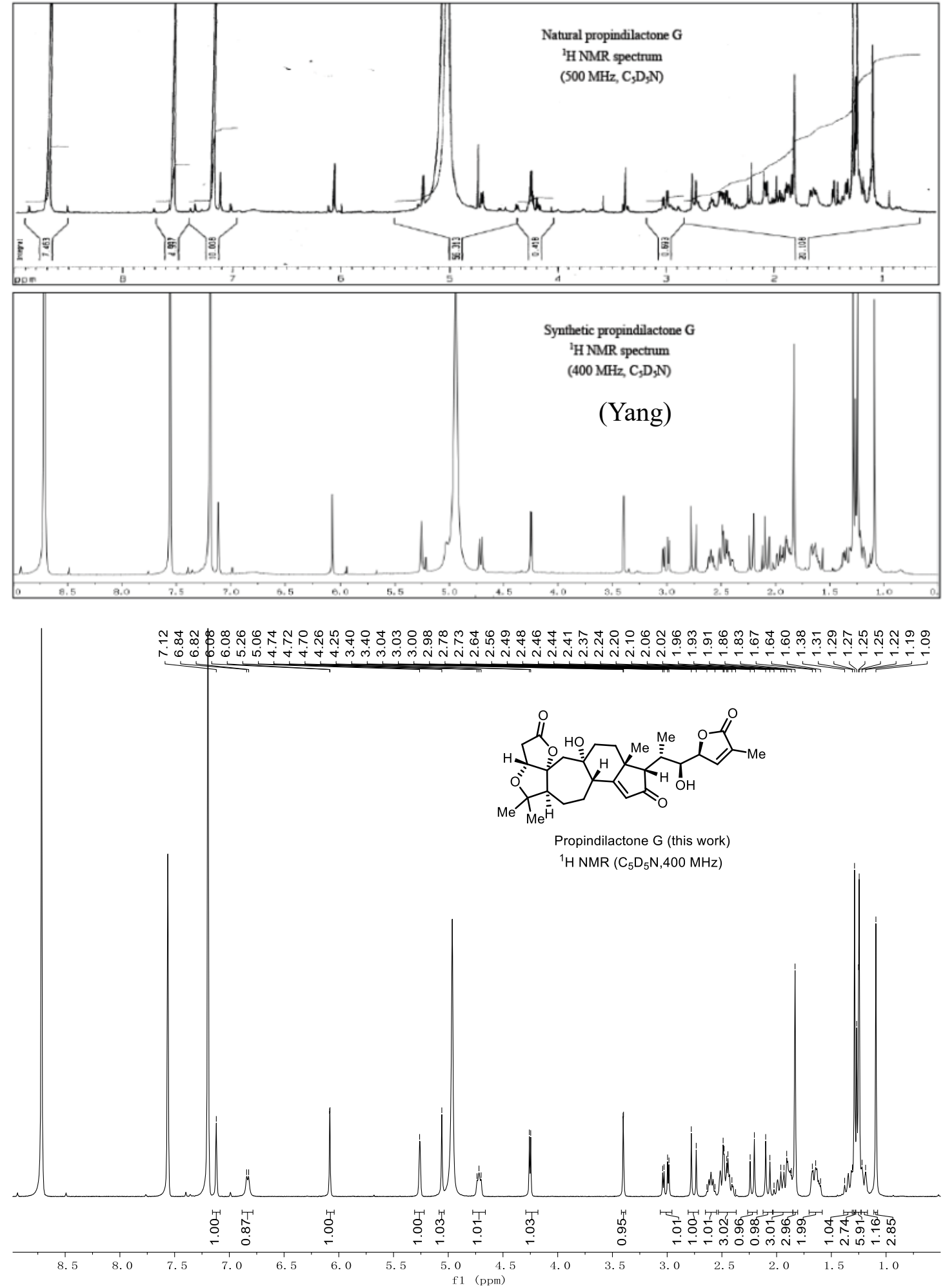
Note: When $\mathrm{D}_{2} \mathrm{O}$ was added to the synthetic sample of propindilactone $\mathrm{G}$ in $\mathrm{C}_{5} \mathrm{D}_{5} \mathrm{~N}$, the ${ }^{1} \mathrm{H}$ NMR signals of $\mathrm{C} 22-\mathrm{OH}(\delta 6.83 \mathrm{ppm})$ and $\mathrm{C} 9-\mathrm{OH}(\delta 5.06 \mathrm{ppm})$ disappeared and the signal of $\mathrm{C} 22-\mathrm{H}$ changed from triplet $(J=7.5 \mathrm{~Hz})$ to doublet $(J=8.8 \mathrm{~Hz})$.
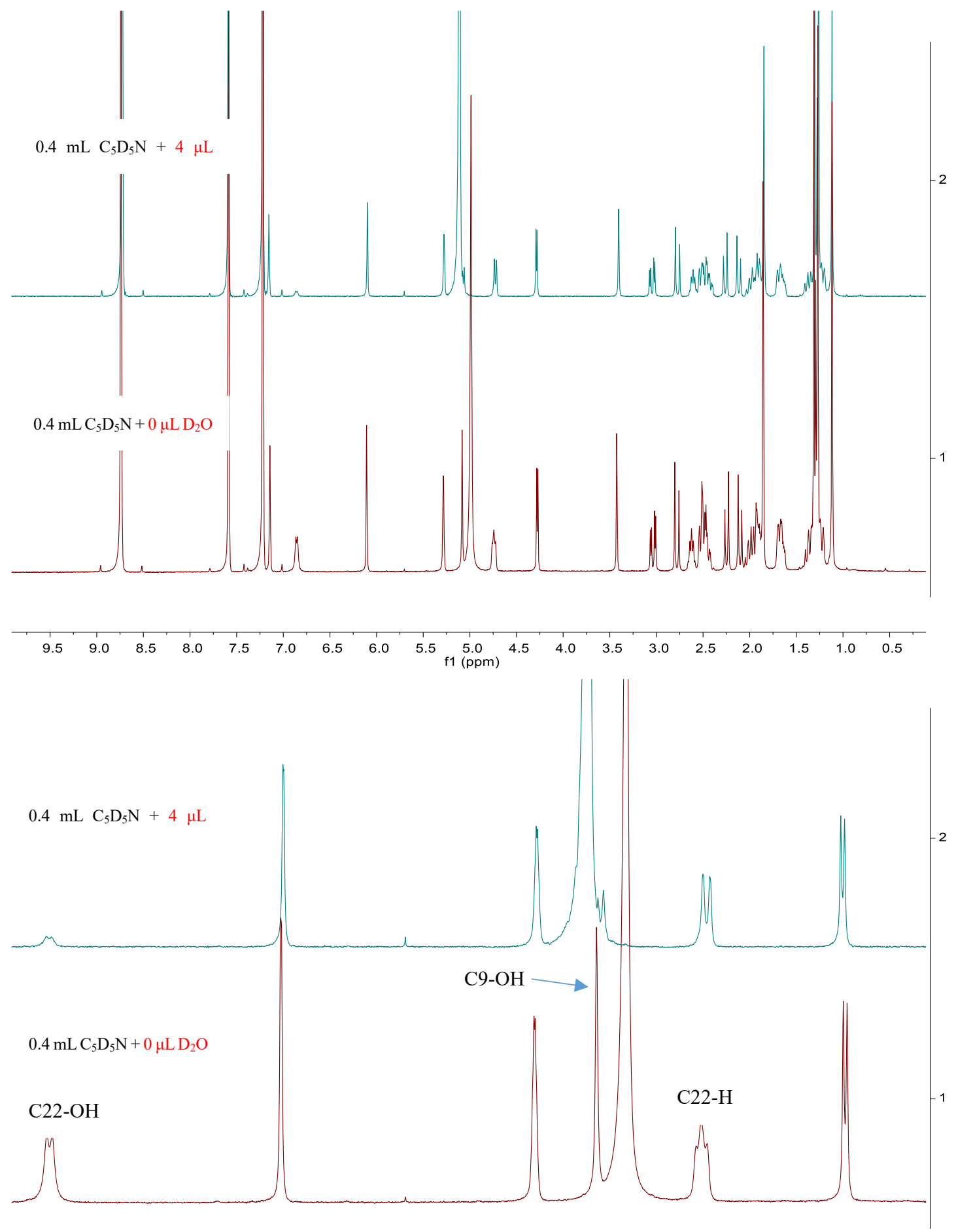
$\begin{array}{lllllllllllllllllllllllllllllllllllllllllll}6.9 & 6.8 & 6.7 & 6.6 & 6.5 & 6.4 & 6.3 & 6.2 & 6.1 & 6.0 & 5.9 & 5.8 & 5.7 & 5.6 & 5.5 & 5.4 & 5.3 & 5.2 & 5.1 & 5.0 & 4.9 & 4.8 & 4.7 & 4.6 & 4.5 & 4.4 & 4.3 & 4.2 & 4.1\end{array}$ 


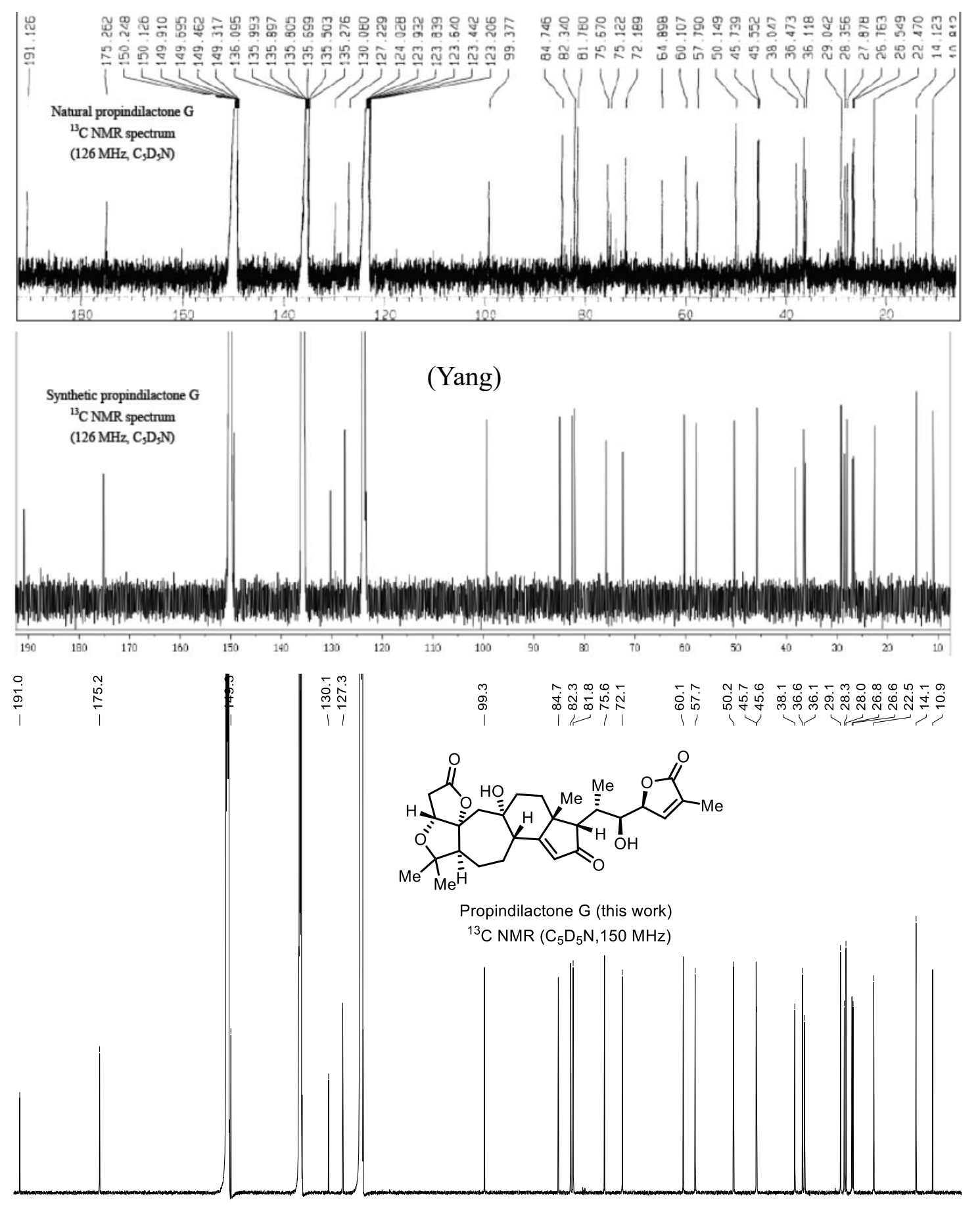

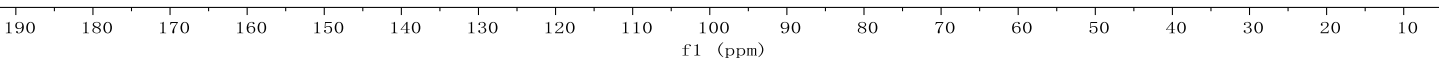




\section{X-ray crystallographic data for compounds 18 and 24}

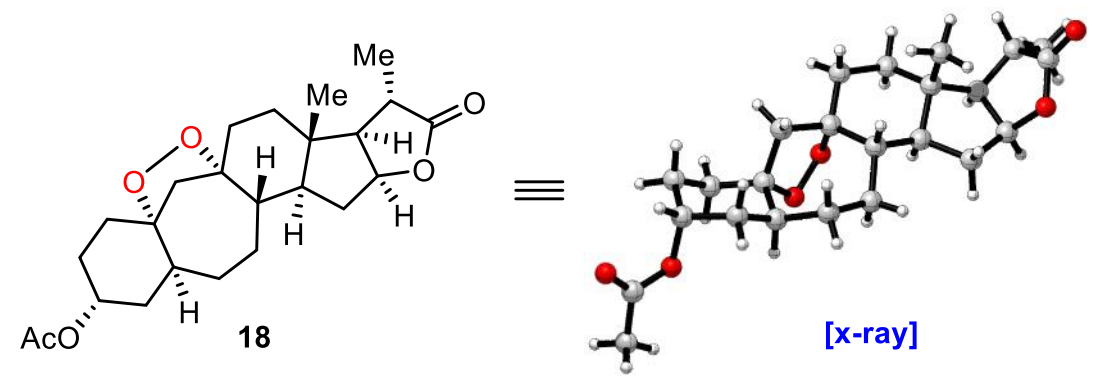

Table S1. Crystal data and structure refinement for CCDC 1970045

Identification code

Empirical formula

Formula weight

Temperature

Wavelength

Crystal system

Space group

Unit cell dimensions

Volume

Z

Density (calculated)

Absorption coefficient

$\mathrm{F}(000)$

Crystal size

Theta range for data collection

Index ranges

Reflections collected

Independent reflections

Completeness to theta $=67.679^{\circ}$

Absorption correction

Max. and min. transmission

Refinement method

Data / restraints / parameters

Goodness-of-fit on $\mathrm{F}^{2}$

Final $\mathrm{R}$ indices [I $>2 \operatorname{sigma}(\mathrm{I})]$

$\mathrm{R}$ indices (all data)
CCDC 1970045

C24 H34 O6

418.51

293(2) K

$1.54178 \AA$

Orthorhombic

P 212121

$$
\begin{array}{ll}
\mathrm{a}=5.7822(5) \AA & \mathrm{a}=90^{\circ} . \\
\mathrm{b}=14.2070(11) \AA & \mathrm{b}=90^{\circ} . \\
\mathrm{c}=26.733(2) \AA & \mathrm{g}=90^{\circ} .
\end{array}
$$

2196.1(3) $\AA^{3}$

4

$1.266 \mathrm{Mg} / \mathrm{m}^{3}$

$0.729 \mathrm{~mm}^{-1}$

904

$0.190 \times 0.100 \times 0.060 \mathrm{~mm}^{3}$

3.523 to $64.979^{\circ}$.

$-5<=\mathrm{h}<=6,-16<=\mathrm{k}<=16,-31<=\mathrm{l}<=29$

10368

$3676[\mathrm{R}(\mathrm{int})=0.0782]$

$93.3 \%$

Semi-empirical from equivalents

0.7533 and 0.4242

Full-matrix least-squares on $\mathrm{F}^{2}$

3676 / 0 / 274

1.037

$\mathrm{R} 1=0.0805, \mathrm{wR} 2=0.2093$

$\mathrm{R} 1=0.0930, \mathrm{wR} 2=0.2270$ 
Absolute structure parameter

Extinction coefficient

Largest diff. peak and hole
$0.3(2)$

$\mathrm{n} / \mathrm{a}$

0.350 and -0.308 e. $\AA^{-3}$ 

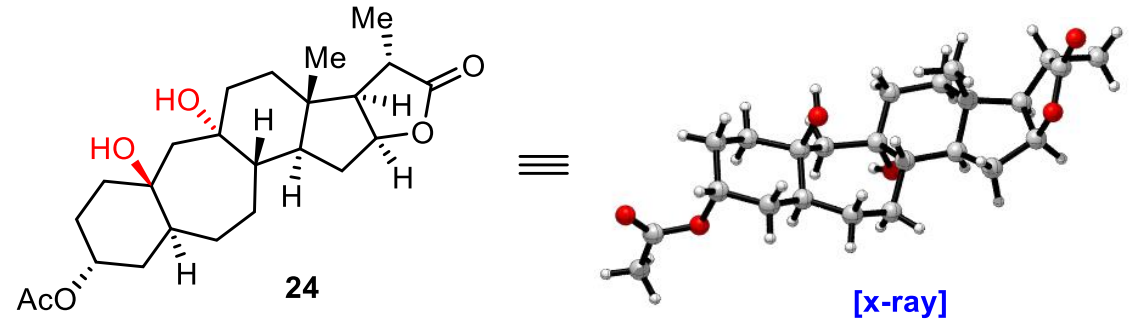

Table S2. Crystal data and structure refinement for CCDC 1970046

Identification code

Empirical formula

Formula weight

Temperature

Wavelength

Crystal system

Space group

Unit cell dimensions

Volume

Z

Density (calculated)

Absorption coefficient

$\mathrm{F}(000)$

Crystal size

Theta range for data collection

Index ranges

Reflections collected

Independent reflections

Completeness to theta $=67.679^{\circ}$

Absorption correction

Max. and min. transmission

Refinement method

Data / restraints / parameters

Goodness-of-fit on $\mathrm{F}^{2}$

Final $\mathrm{R}$ indices [I $>2 \operatorname{sigma}(\mathrm{I})]$

$\mathrm{R}$ indices (all data)

Absolute structure parameter
CCDC 1970046

C24 H38 O7

438.54

293(2) K

$1.54178 \AA$

Orthorhombic

P 212121

$a=6.2210(4) \AA$

$\mathrm{a}=90^{\circ}$.

$\mathrm{b}=18.4005(11) \AA$

$\mathrm{b}=90^{\circ}$.

$\mathrm{c}=20.3992(15) \AA$

$\mathrm{g}=90^{\circ}$.

2335.1(3) $\AA^{3}$

4

$1.247 \mathrm{Mg} / \mathrm{m}^{3}$

$0.738 \mathrm{~mm}^{-1}$

952

$0.140 \times 0.100 \times 0.050 \mathrm{~mm}^{3}$

3.234 to $65.462^{\circ}$.

$-7<=\mathrm{h}<=7,-21<=\mathrm{k}<=21,-23<=\mathrm{l}<=24$

15047

$4001[\mathrm{R}(\mathrm{int})=0.2107]$

$94.8 \%$

Semi-empirical from equivalents

0.7533 and 0.4035

Full-matrix least-squares on $\mathrm{F}^{2}$

4001 / 0 / 289

1.044

$\mathrm{R} 1=0.0667, \mathrm{wR} 2=0.1709$

$\mathrm{R} 1=0.1384, \mathrm{wR} 2=0.2302$

$-0.2(3)$ 
Extinction coefficient

Largest diff. peak and hole
$0.0081(15)$

0.343 and -0.290 e. $\AA^{-3}$ 
6. NMR spectra
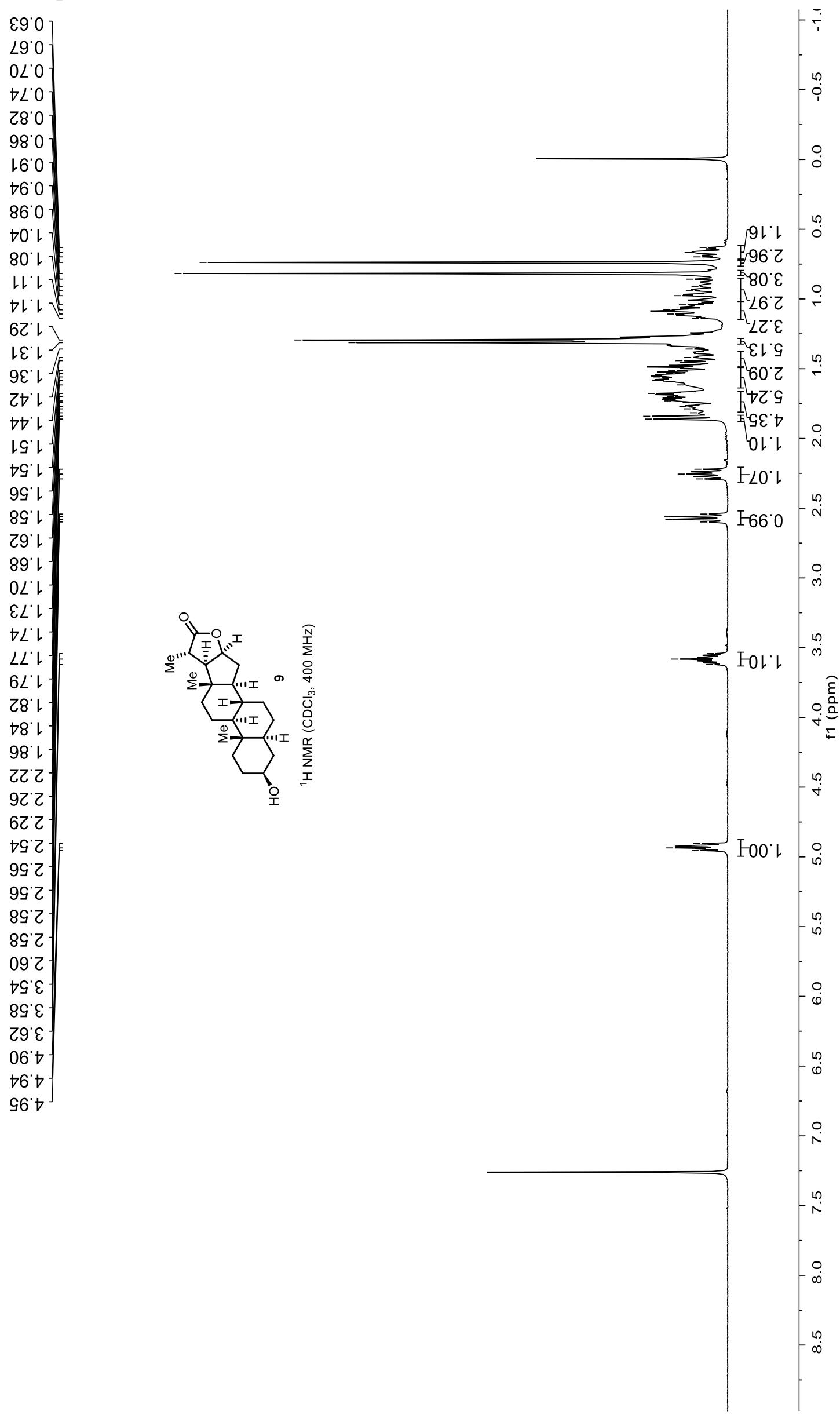


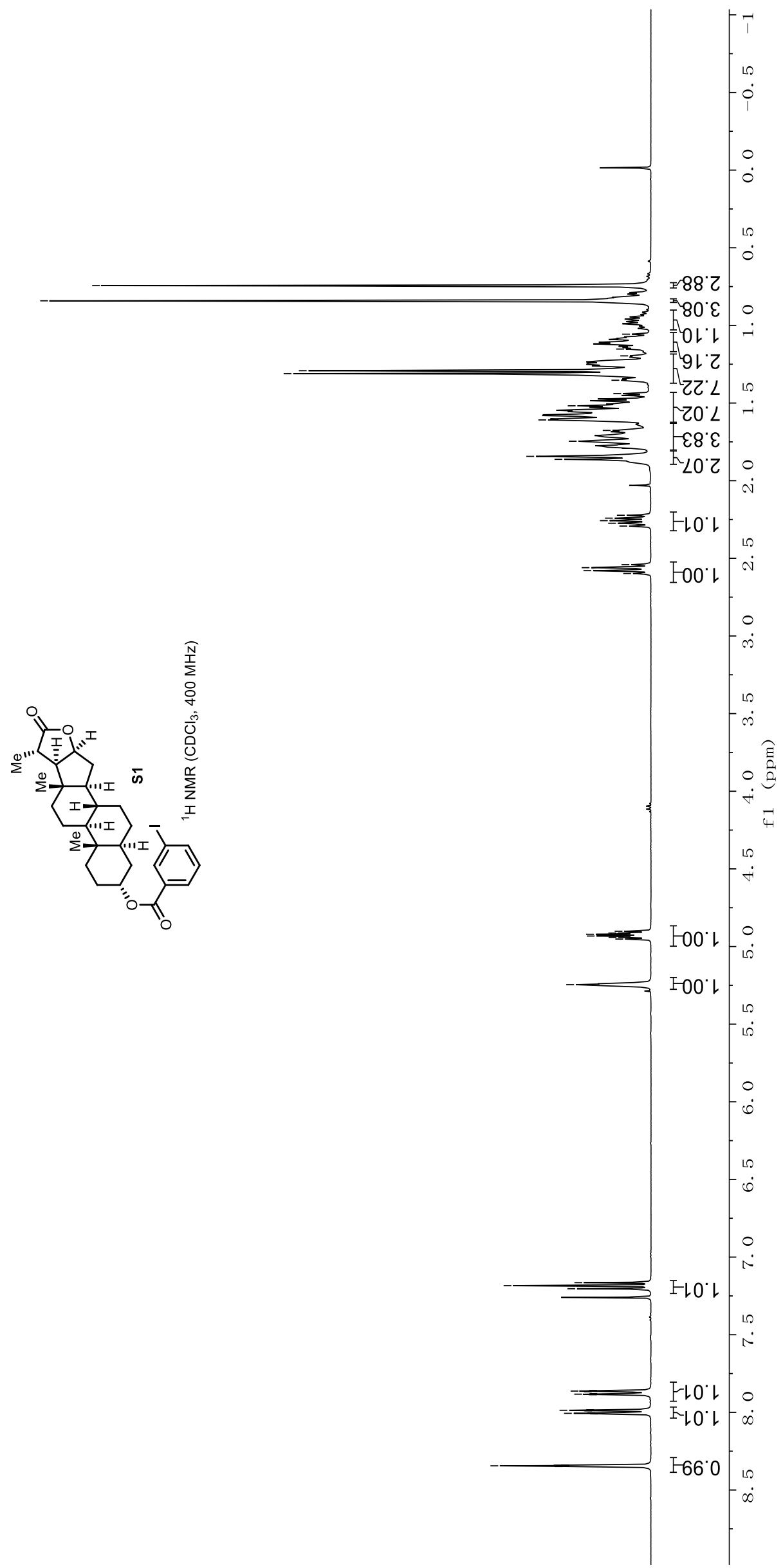




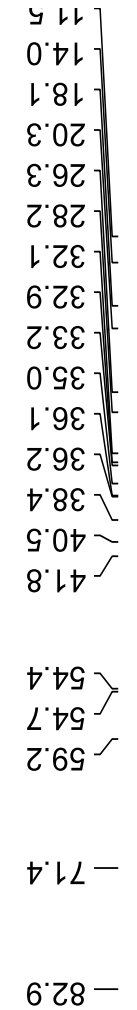

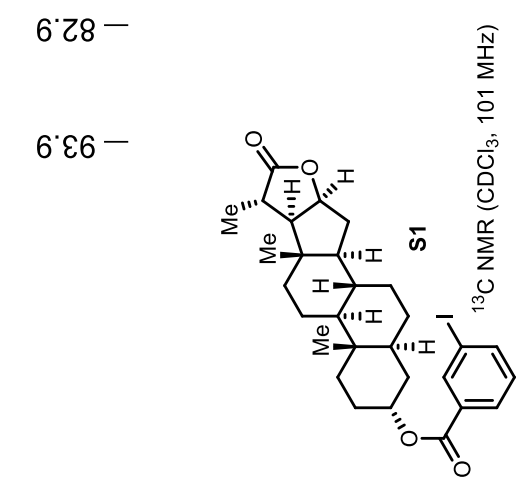

8.8Zレ ح

LOEL

ᄂEع

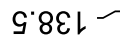

くレレレ

c.t9l-

จ๋เレー

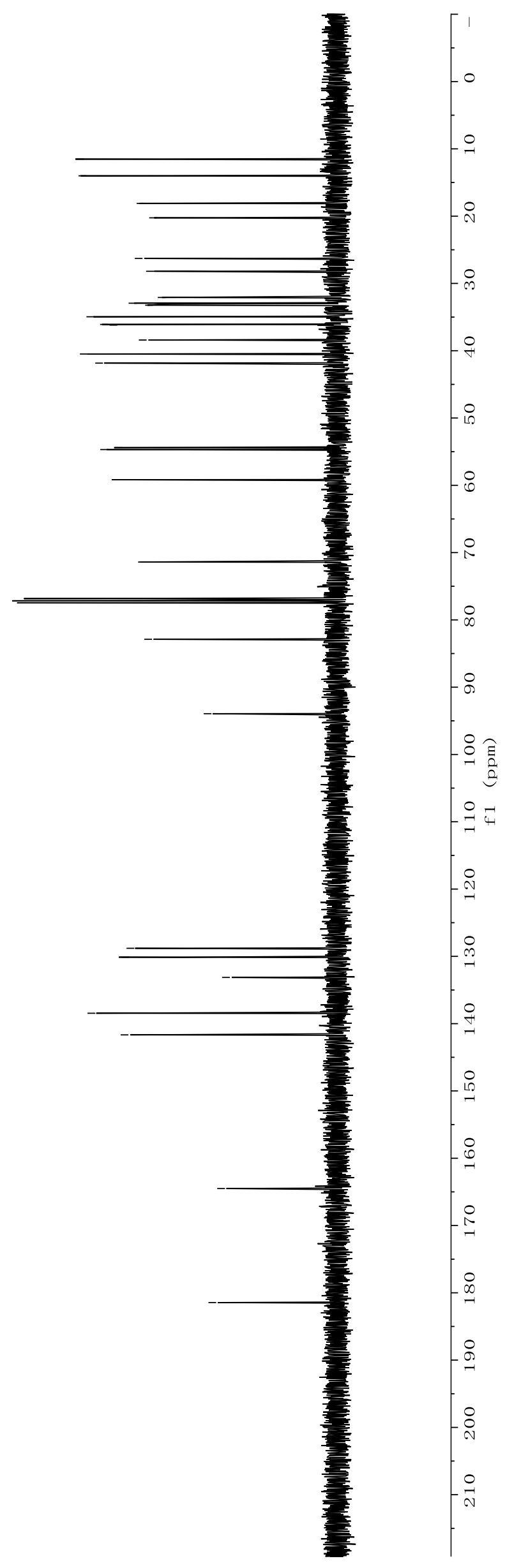




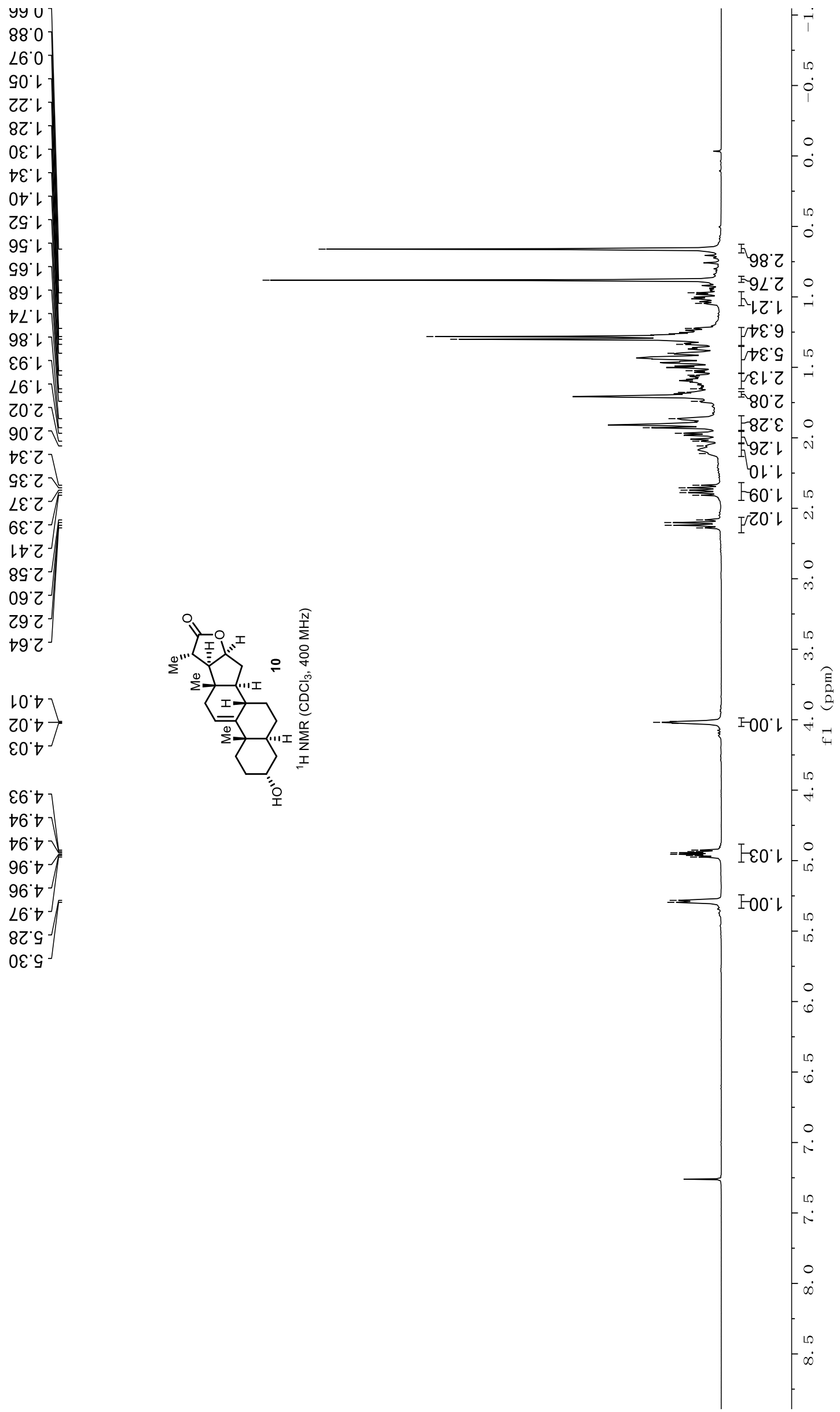


† $\varepsilon\llcorner$ 乙
$6.9 L>$

$6 . \angle L$

$\mathrm{Z} 8 \mathrm{Z}$

ᄂ 62 ᄂ

LOE -

$\tau \cdot \varepsilon \varepsilon$

$\downarrow \downarrow \varepsilon$

$0.9 \varepsilon$

与. $9 \varepsilon$

$\angle L E$

$\mathrm{s} 8 \varepsilon$

0.07

$\varepsilon .0 t$

$\checkmark$ '

8.89

$\varepsilon .99$

乙`६८ -

くナレー

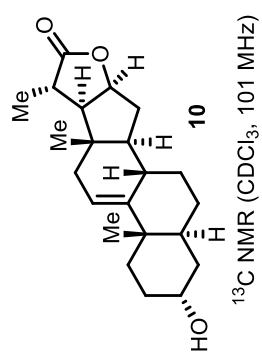

S'டヤレ-

E'เ8L-

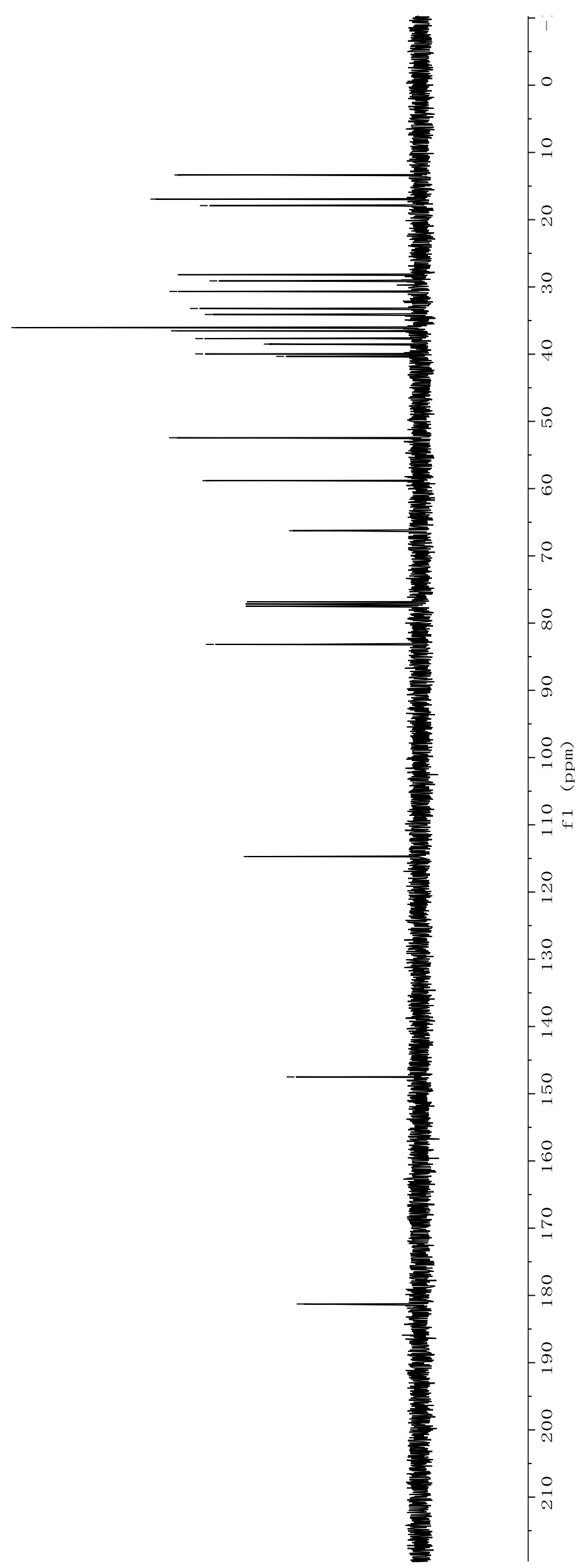




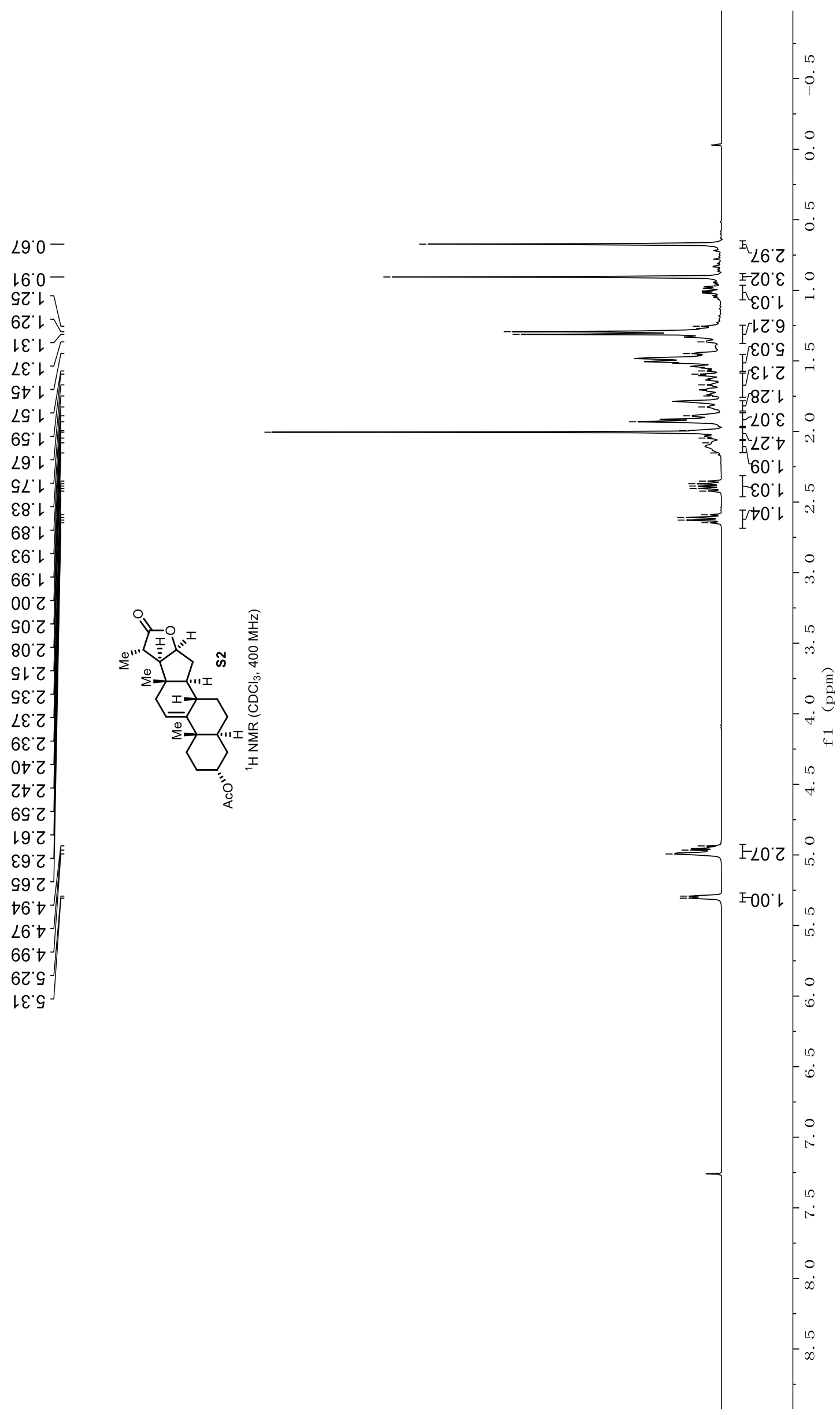




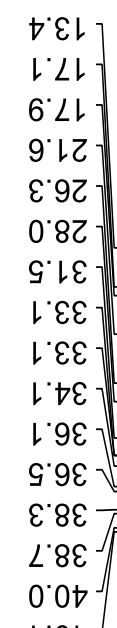

$t .0 t$
$t: 29-$

$6.89-$

$6.69-$

เ'๕8 -

6ナルレー

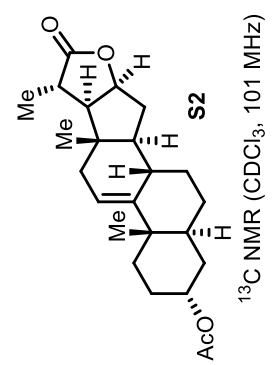

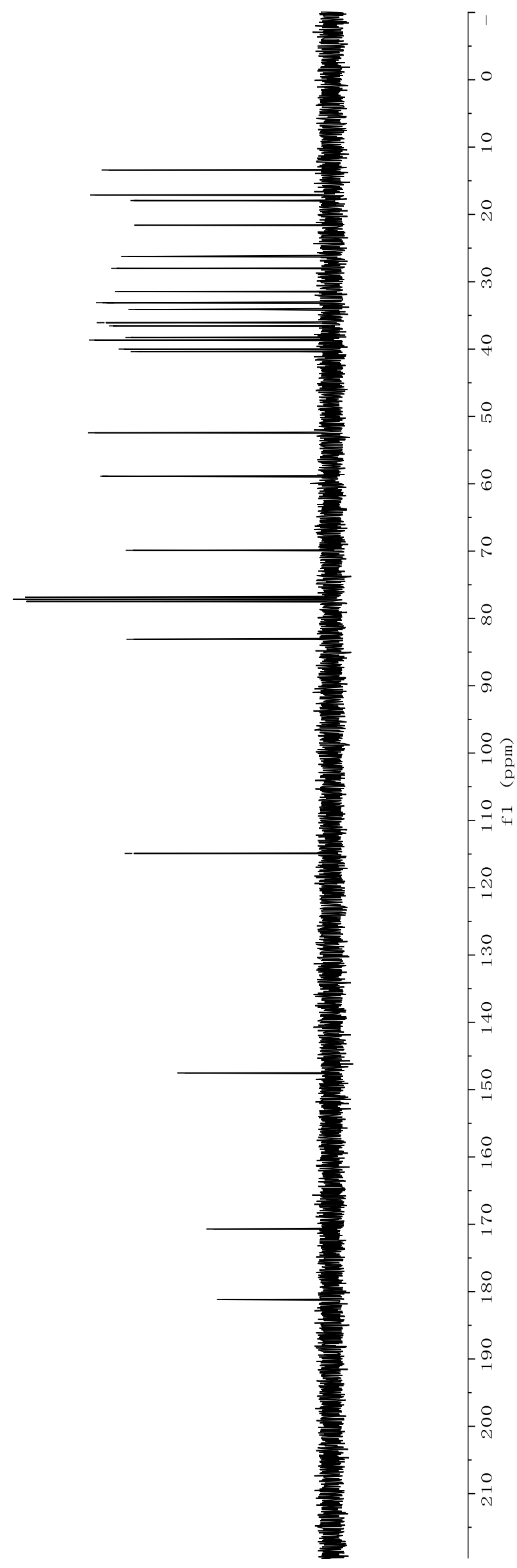




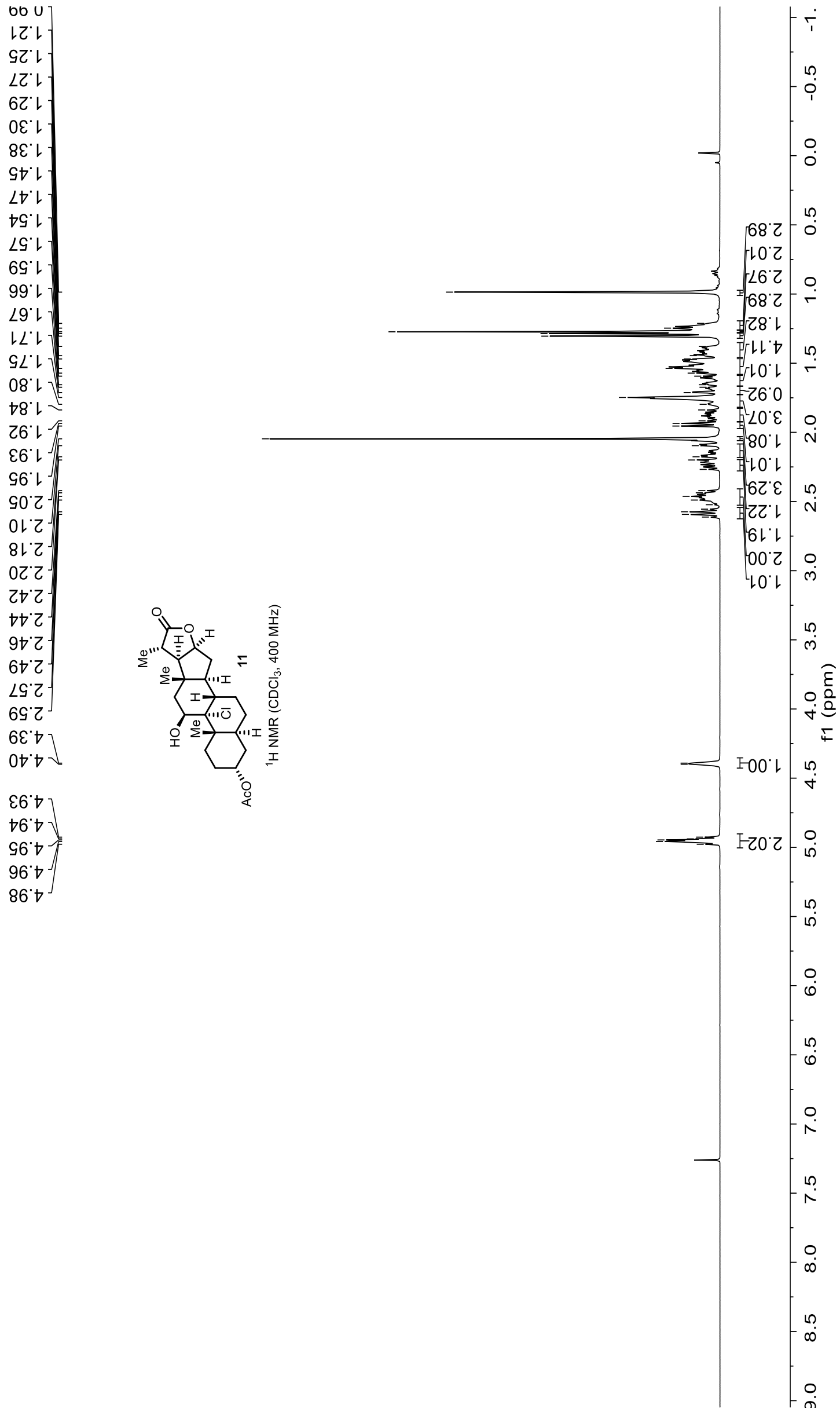




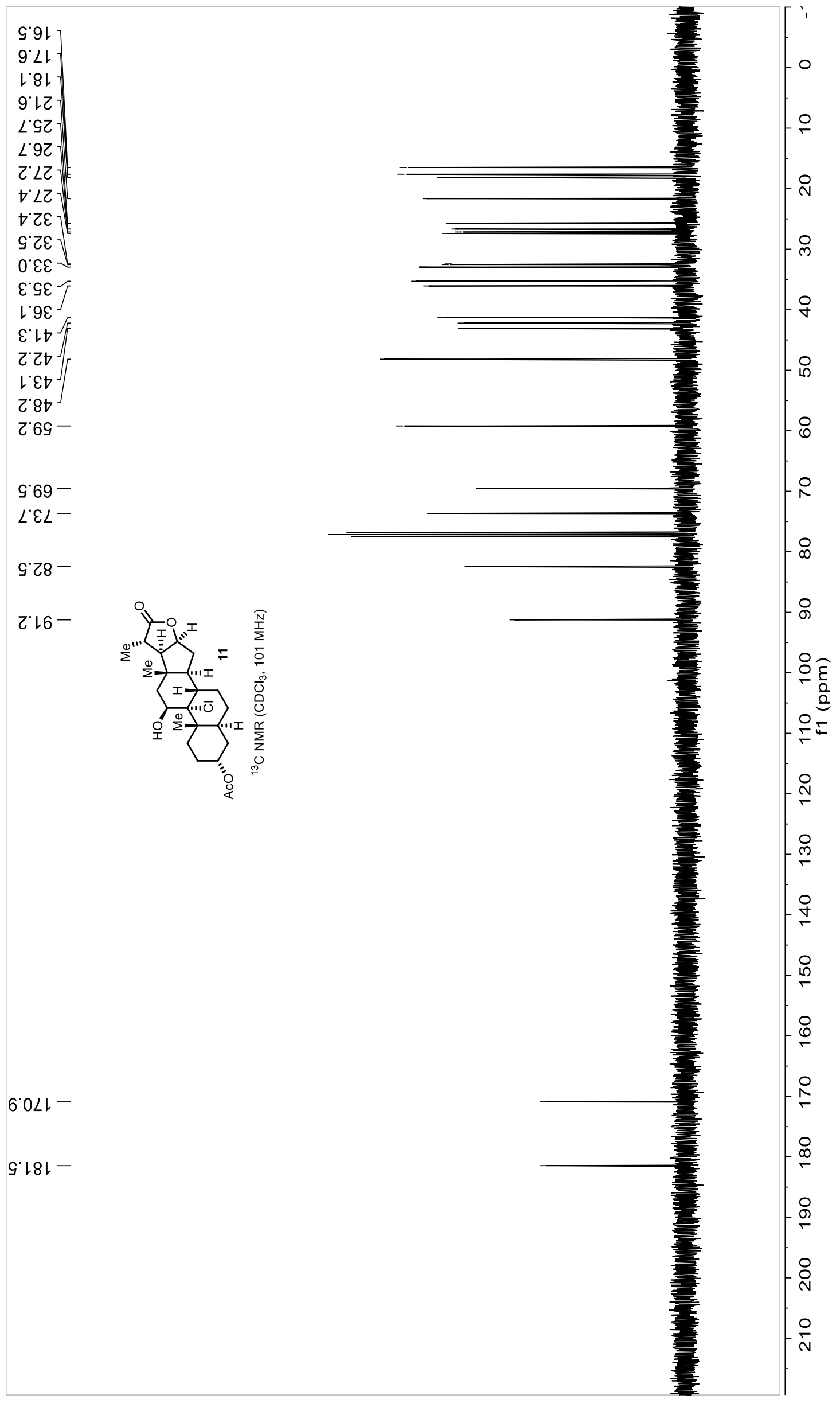




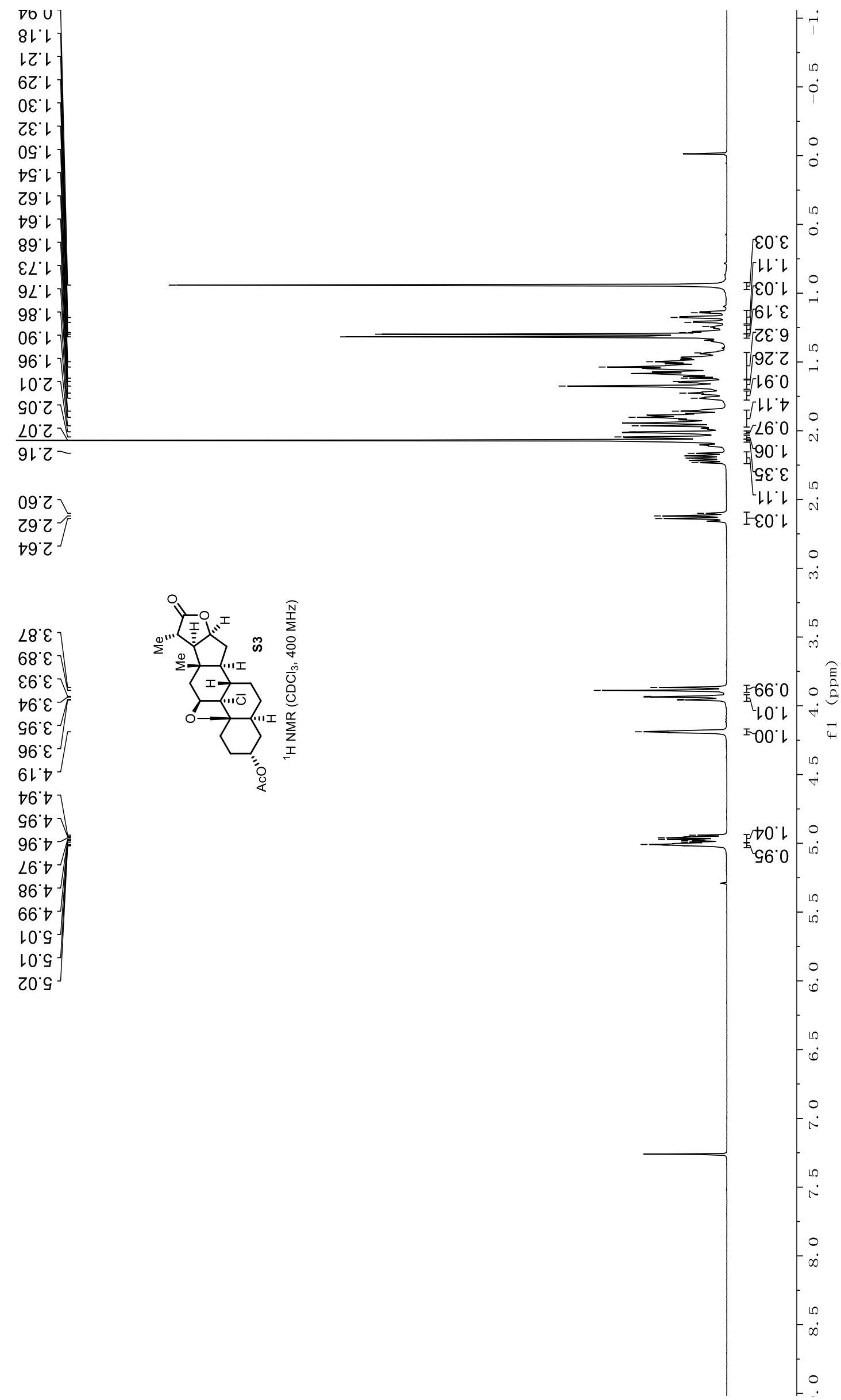



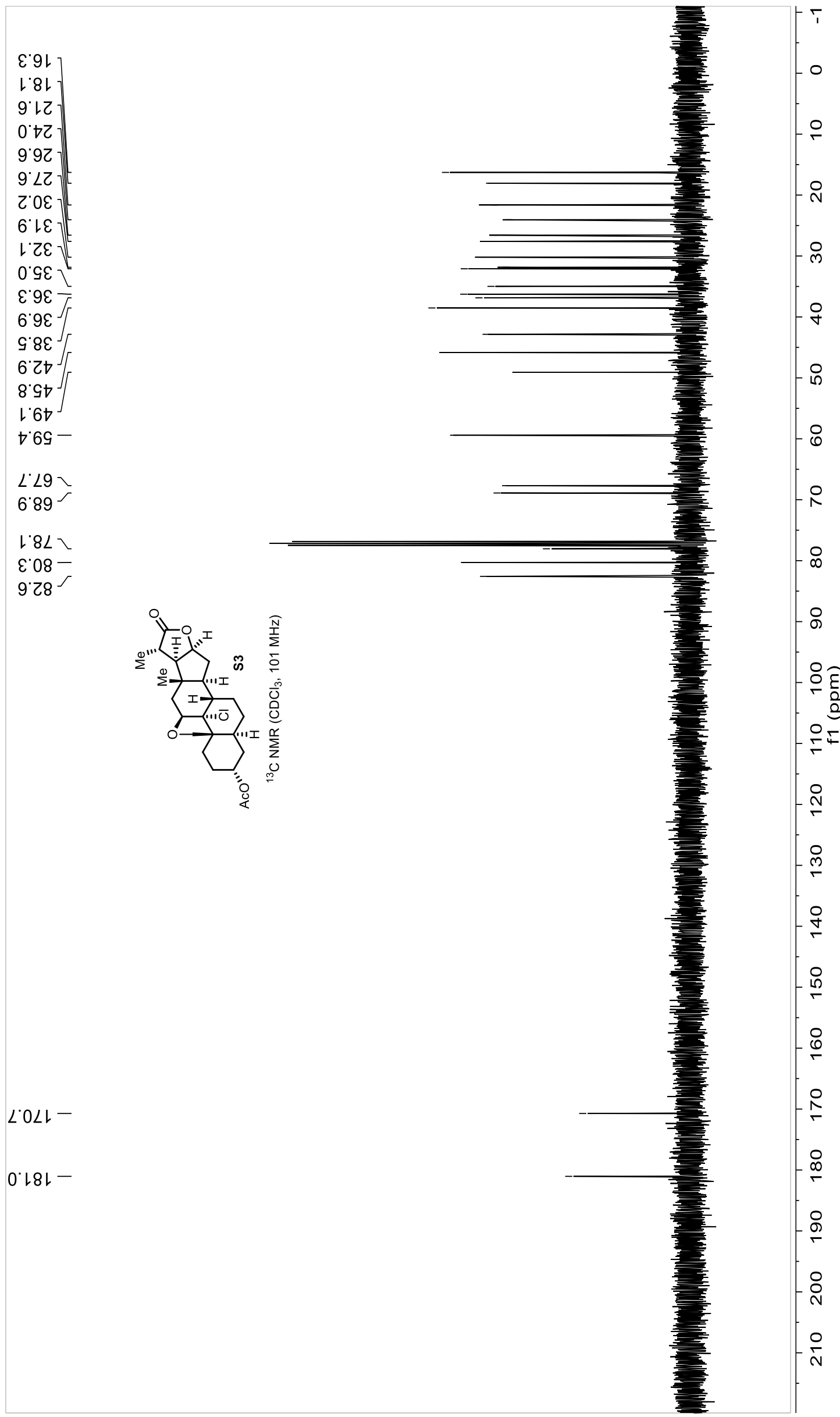


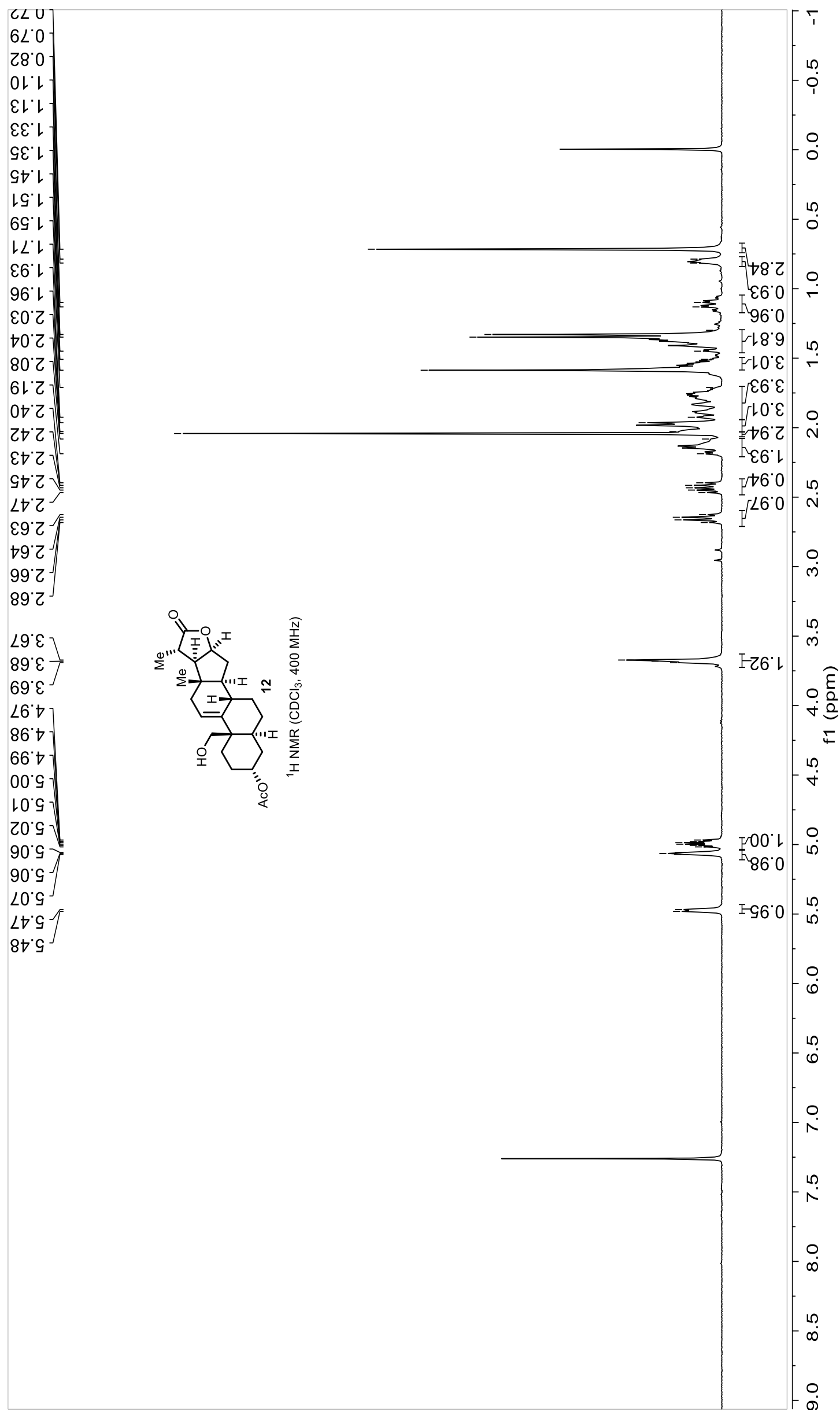




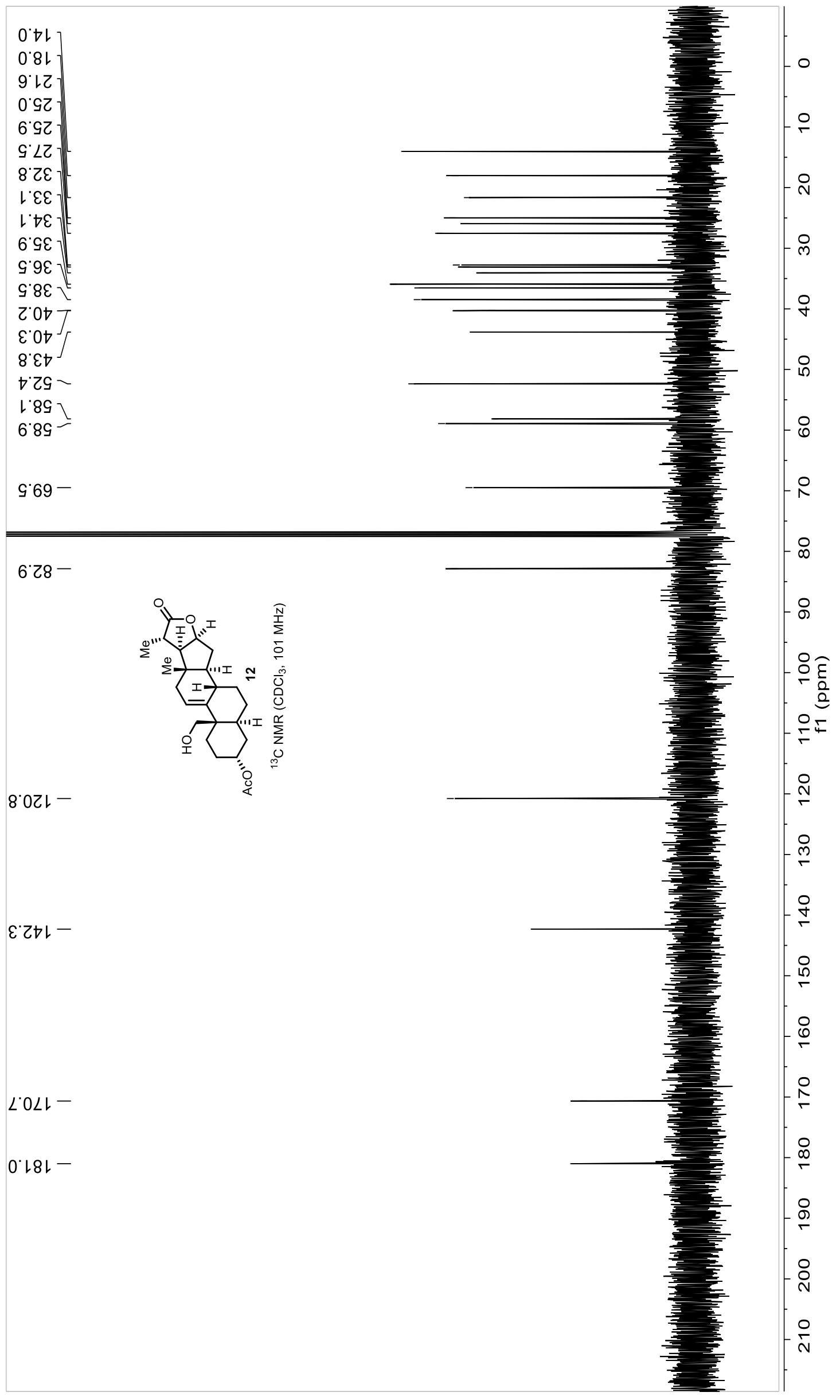



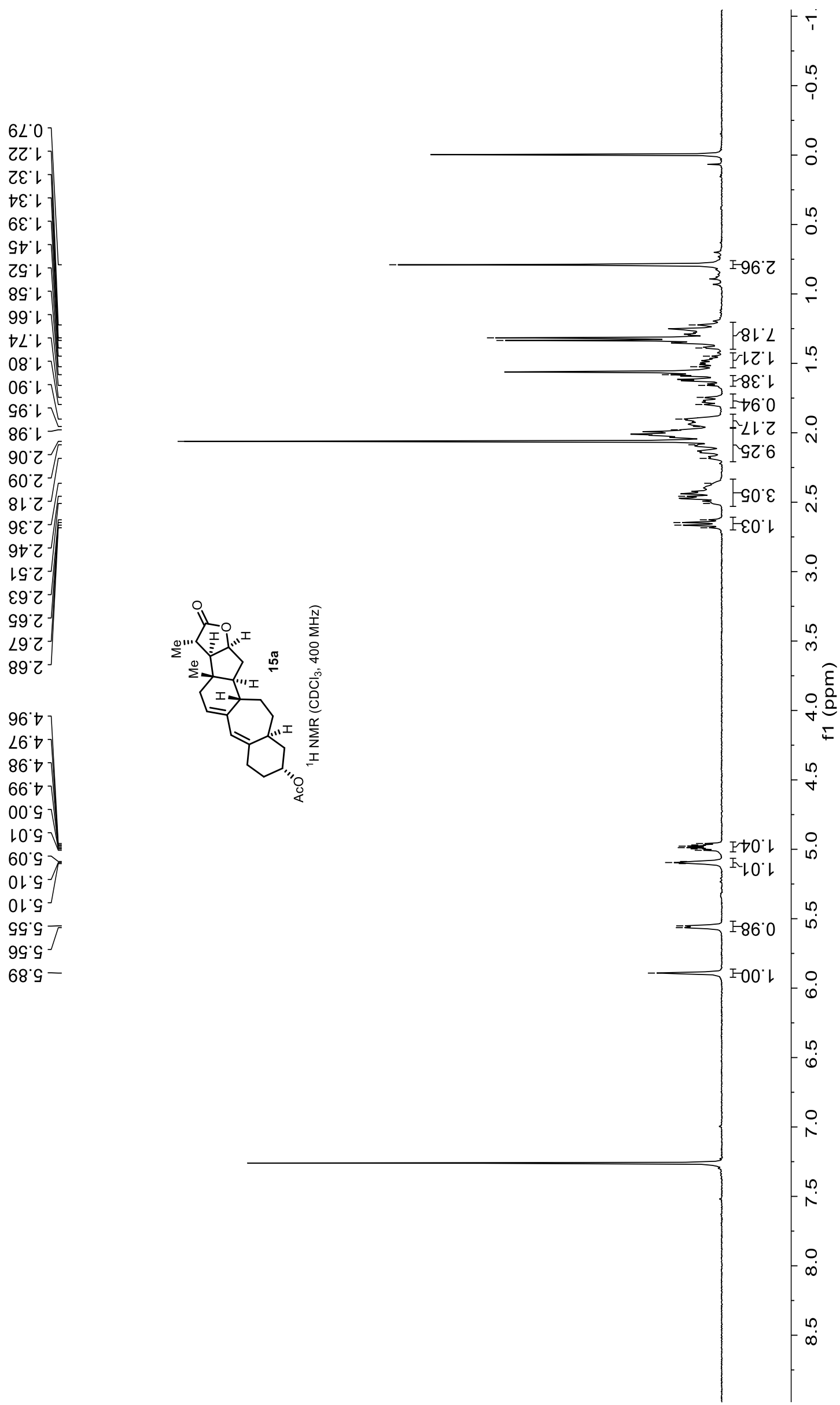


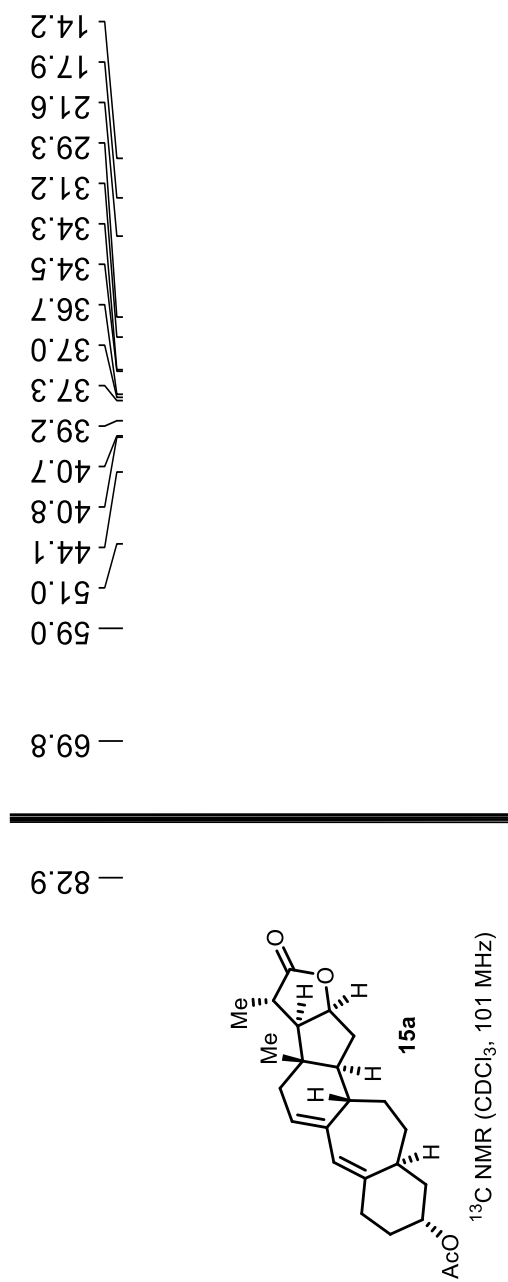

\&'してレ

\&.8Zا

$98 \varepsilon \downarrow$

乙'6ع

8.0LL

0.เレ-

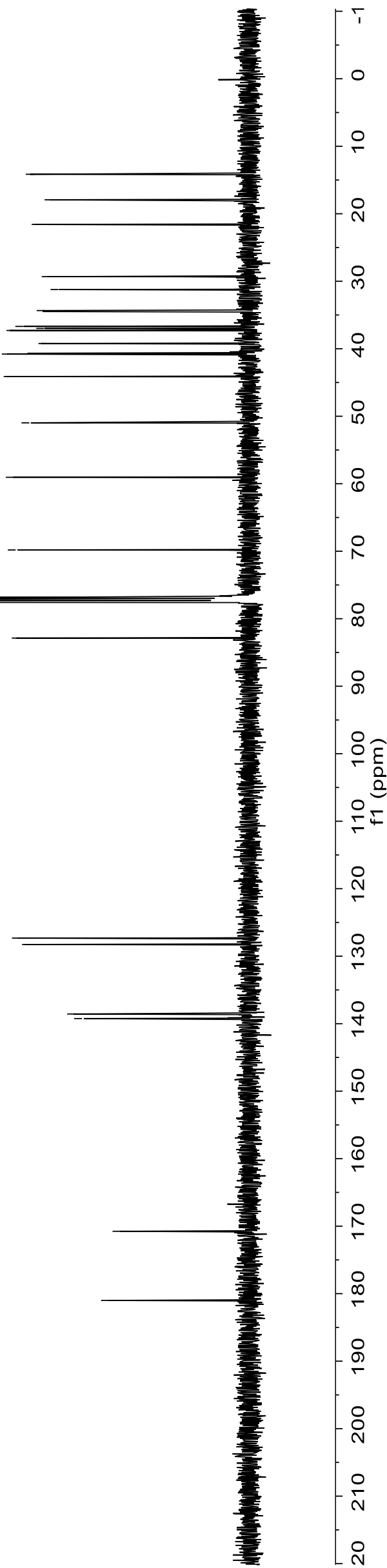


$\varepsilon L^{\circ} 0$

$\left\llcorner\varepsilon^{\prime} \downarrow\right.$

$\varepsilon \varepsilon^{\prime}\llcorner$

$\angle \varepsilon^{\circ} \downarrow$

6ฑเ

Z9'

69'

LLI

$6 L$ '

$\varepsilon 8^{\circ}\llcorner$

$\varepsilon 6^{\circ} \mathrm{L}$

$86^{\circ} \mathrm{L}$

$\supset 0$

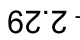

$\nabla \varepsilon \cdot 乙$

$\angle \varepsilon^{\prime} 乙$

$\nabla \nabla 乙$

29 乙

79

s9'

$\angle 9^{\circ} \mathrm{Z}$

$98 \mathrm{Z}$

$88 \mathrm{Z}$

96 乙

$66^{\circ} 乙$

$\nabla 6.7=$

$96^{\circ} \nabla$

$10 \mathrm{~s}$

90.9

$\angle Z{ }^{\circ}$

$8 \mathrm{C}^{\circ}$

$\varepsilon \varepsilon^{\prime} \mathrm{G}$

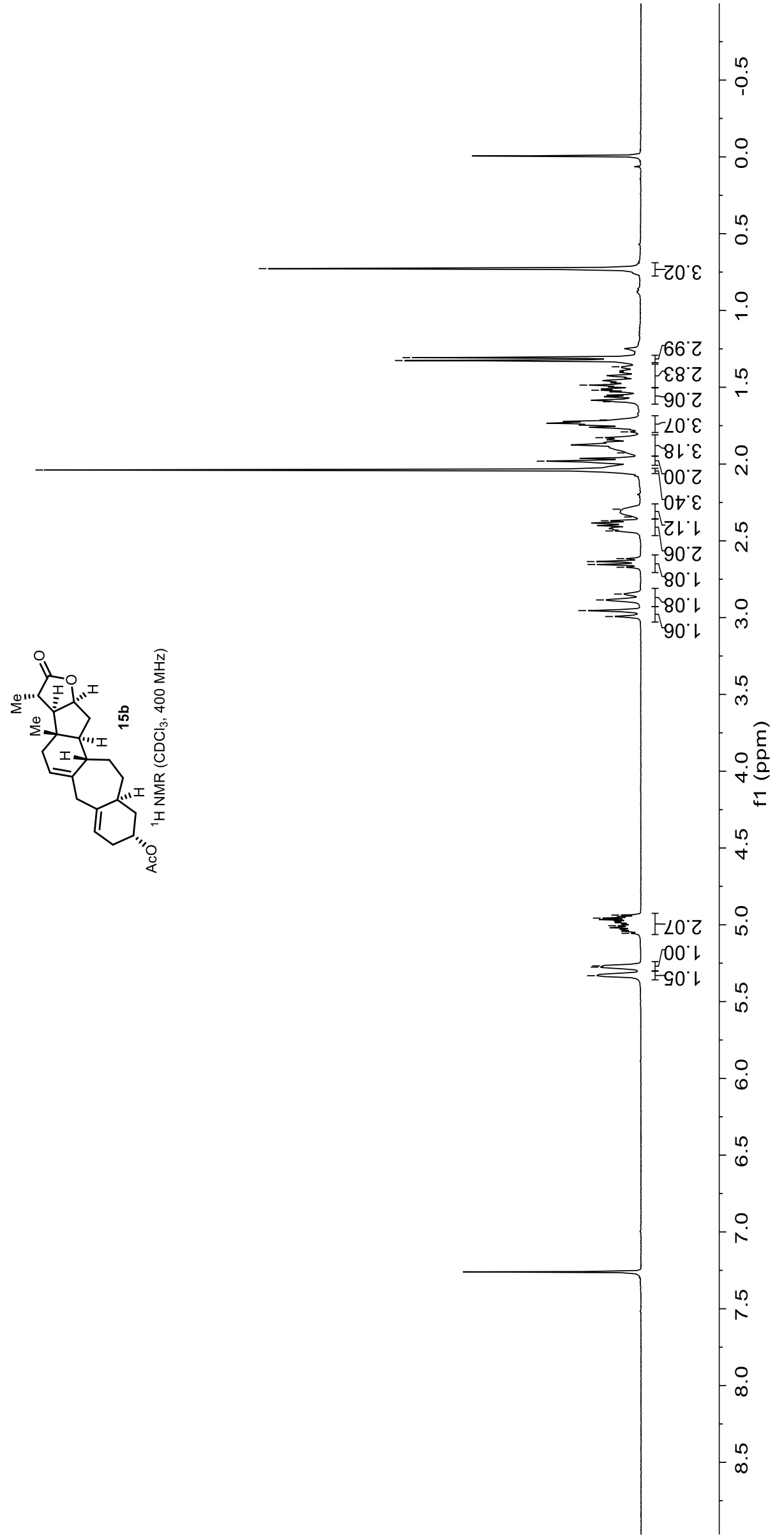




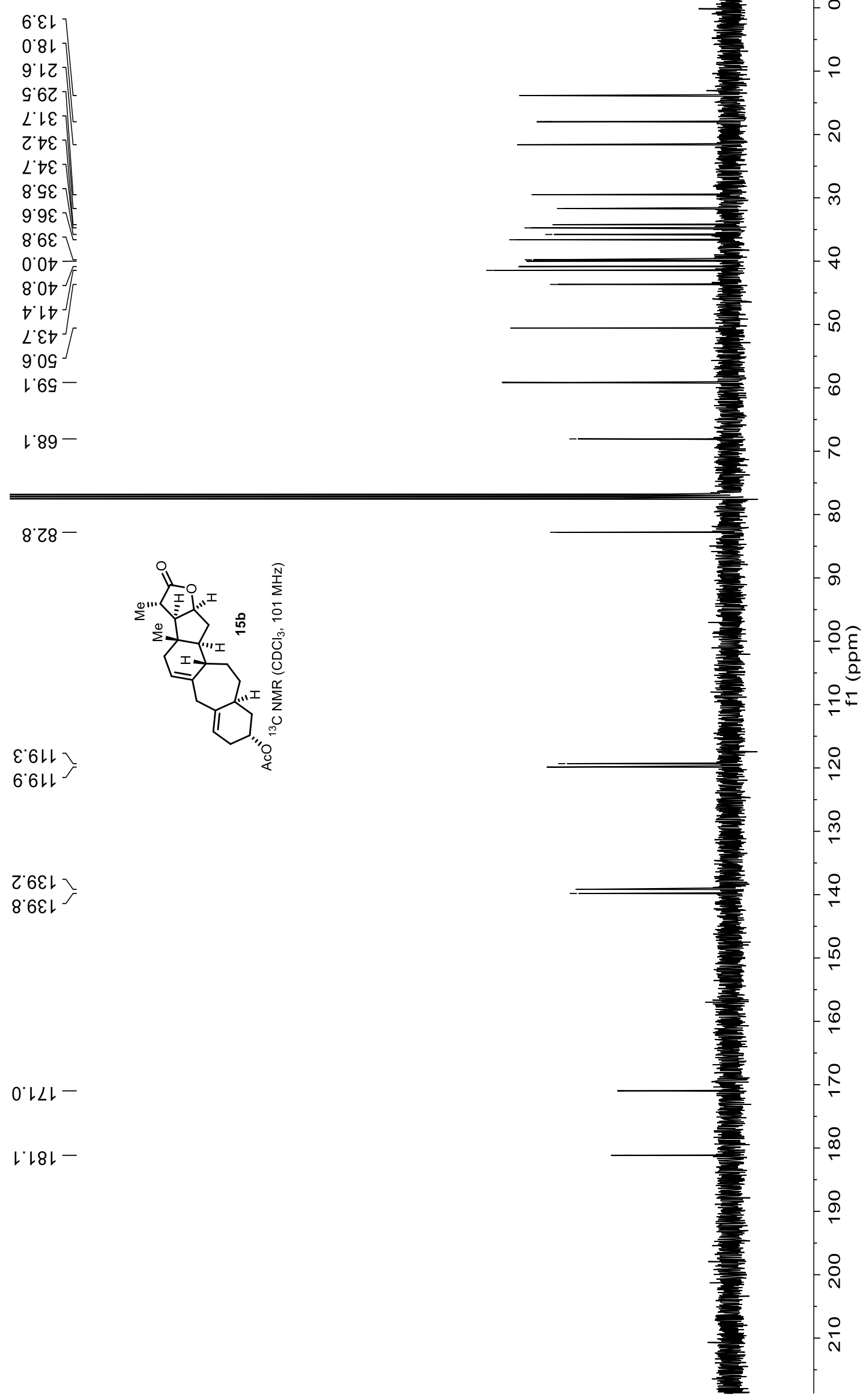



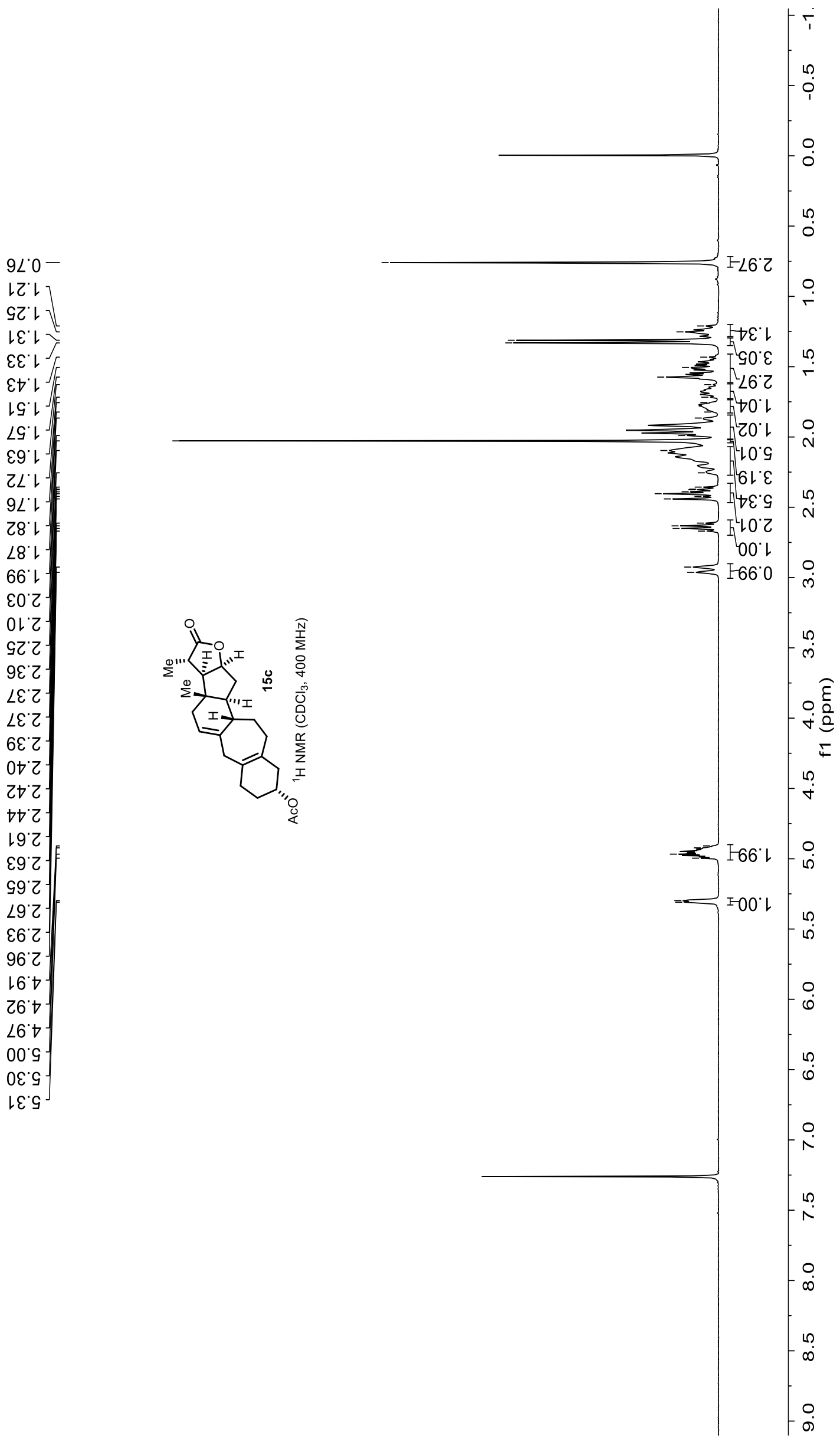


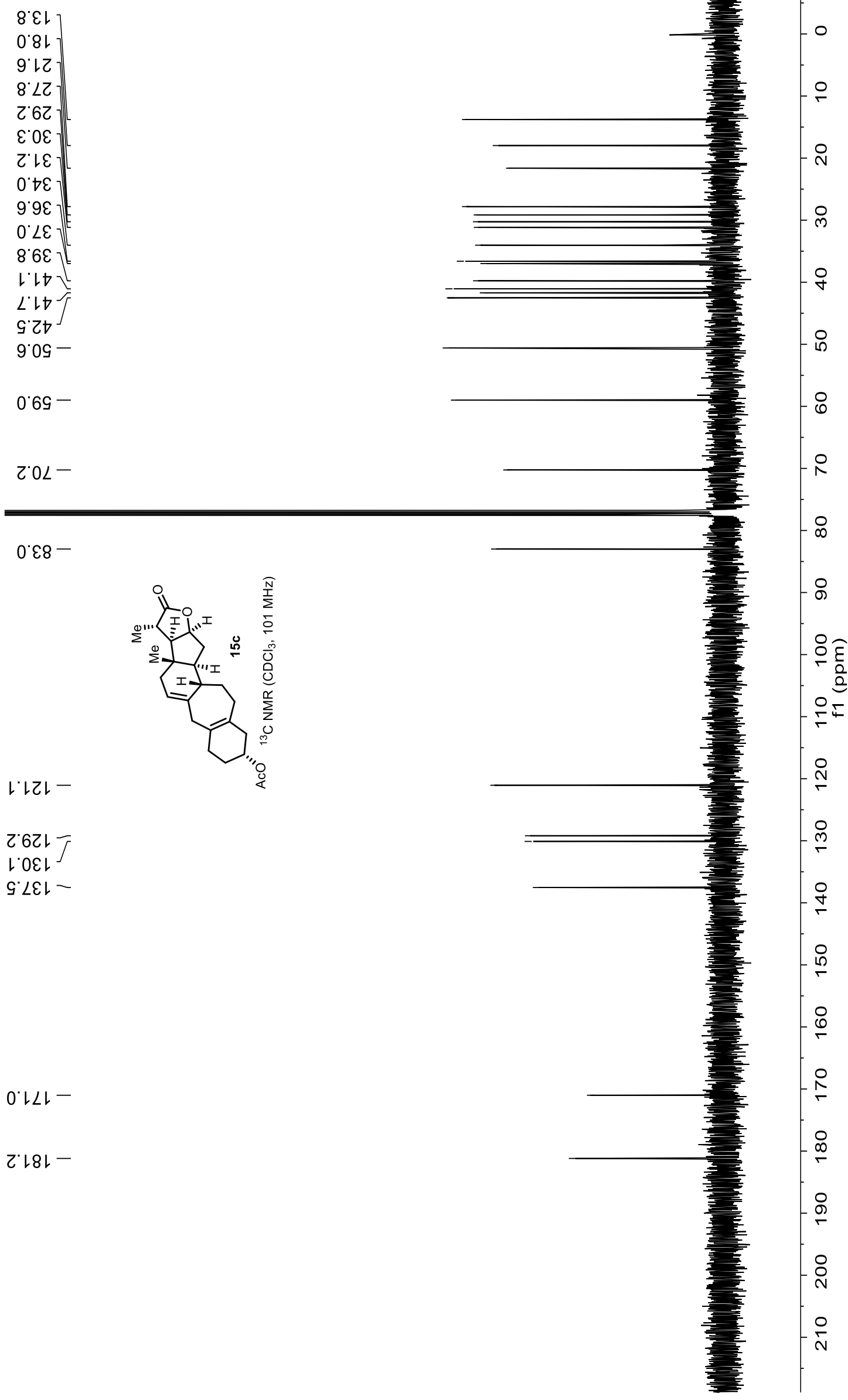




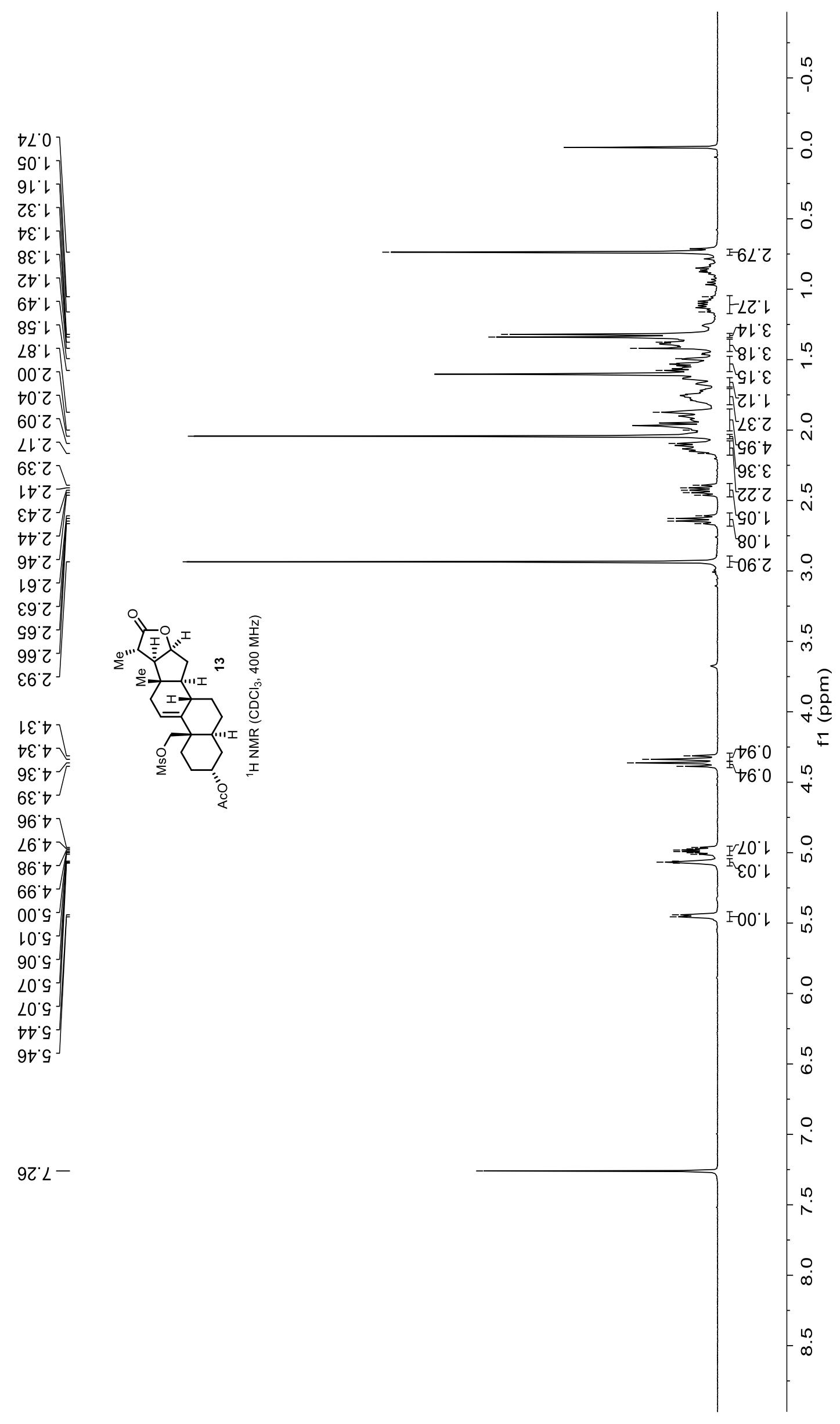



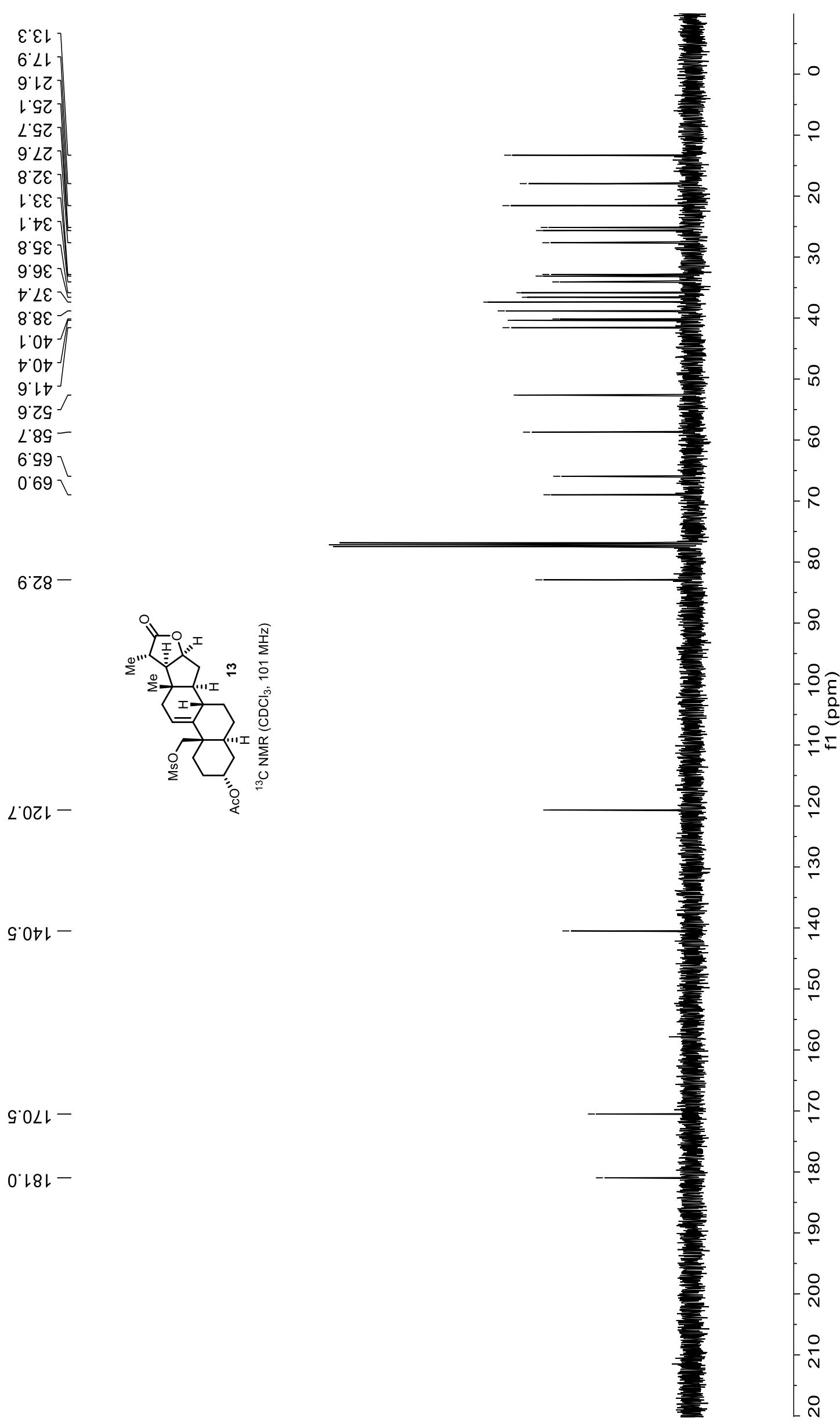


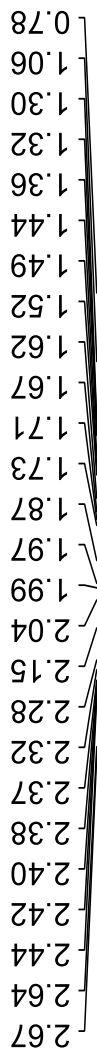

$\varepsilon 6^{\circ} \nabla$

$\nabla 6 . \nabla$

96.

96.

$\angle 6^{\circ} \circ$

86.7

10.9

Z० 9

$\varepsilon 0^{\circ} \mathrm{s}$

乙E' 9

$\varepsilon \varepsilon^{\prime} \mathcal{G}^{\circ}$

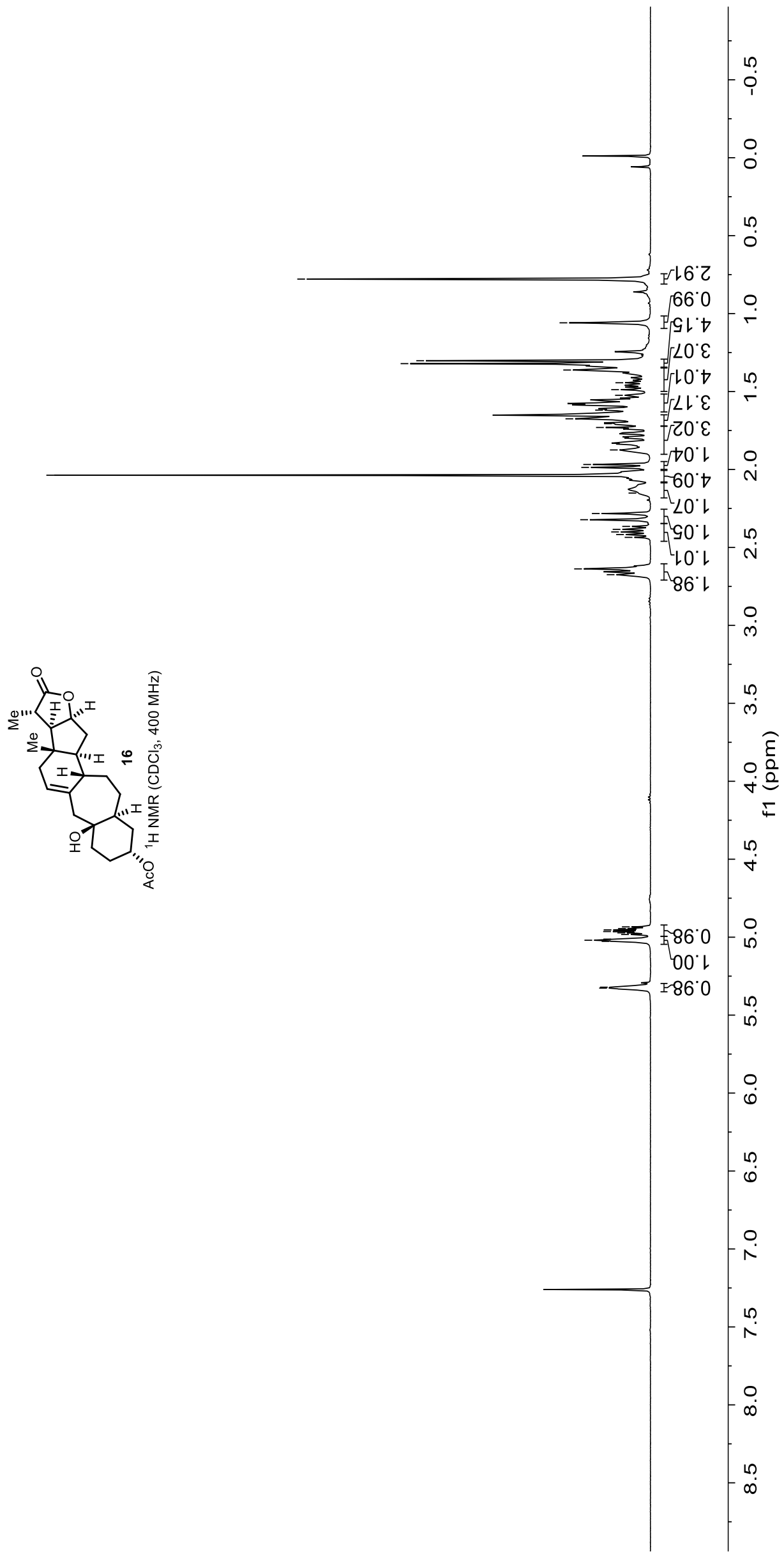




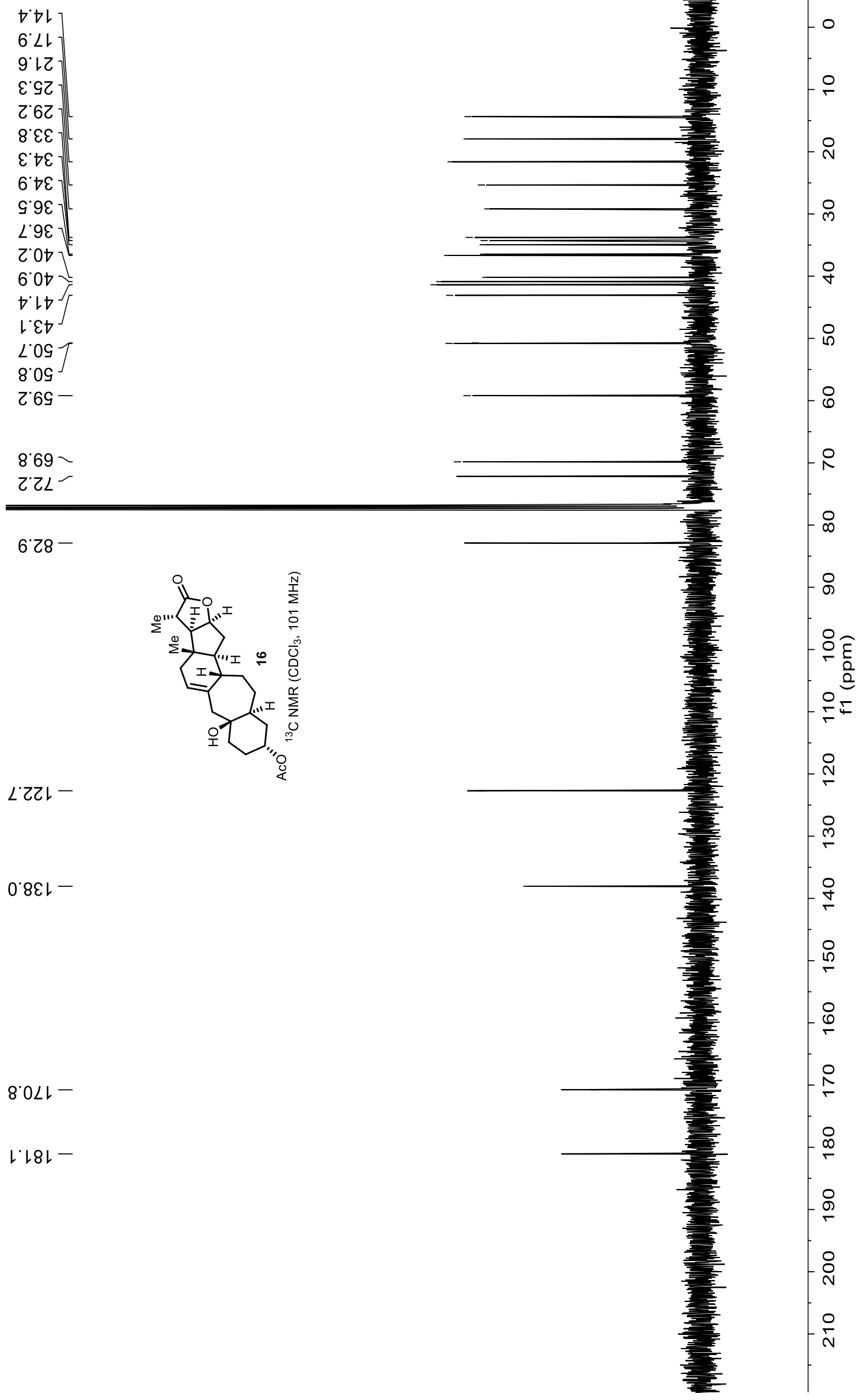




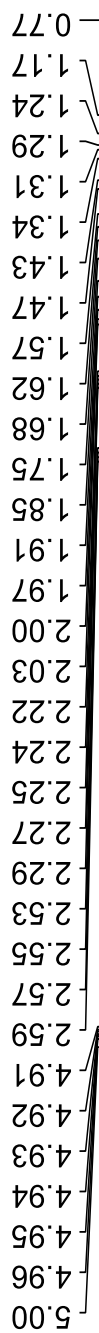

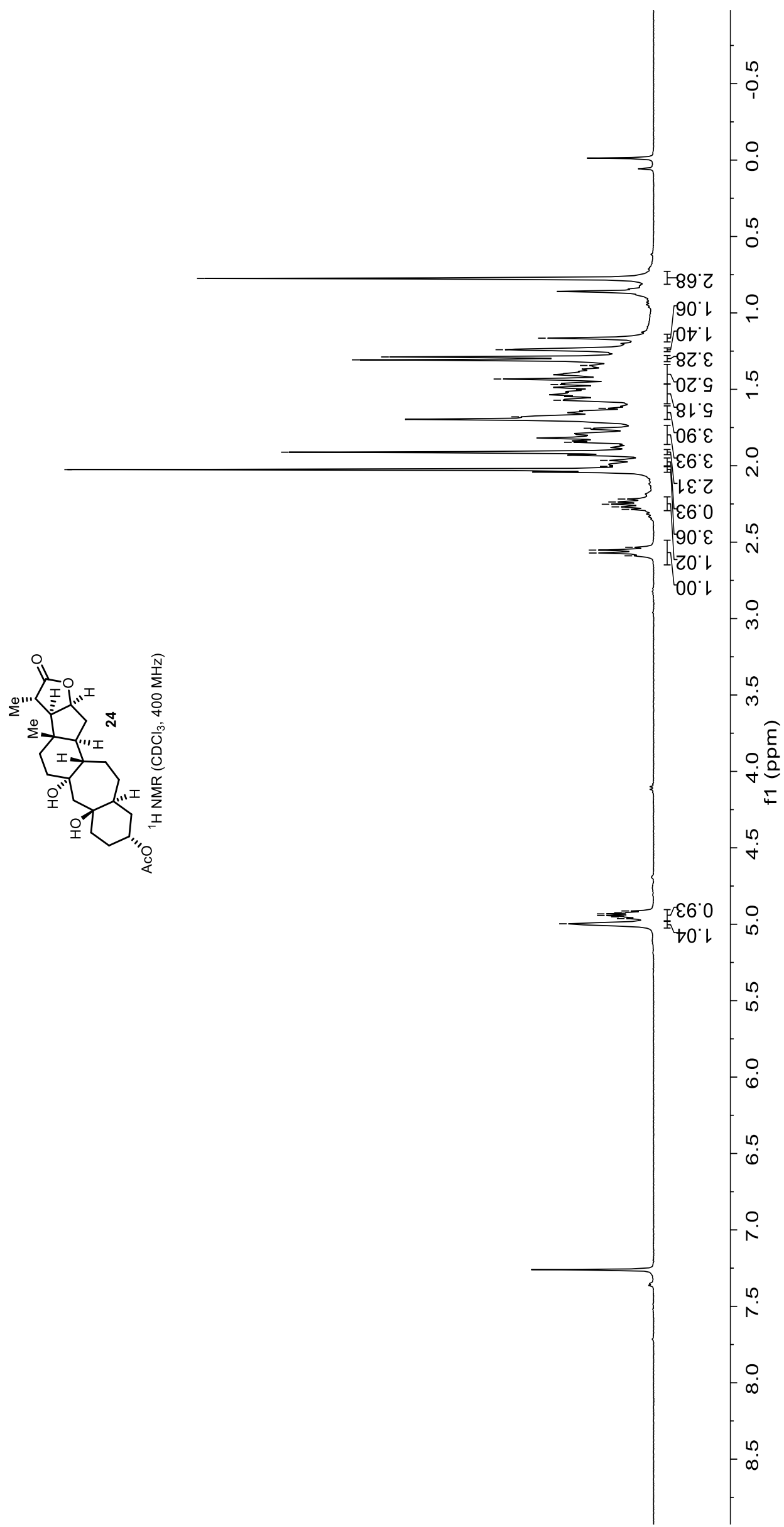



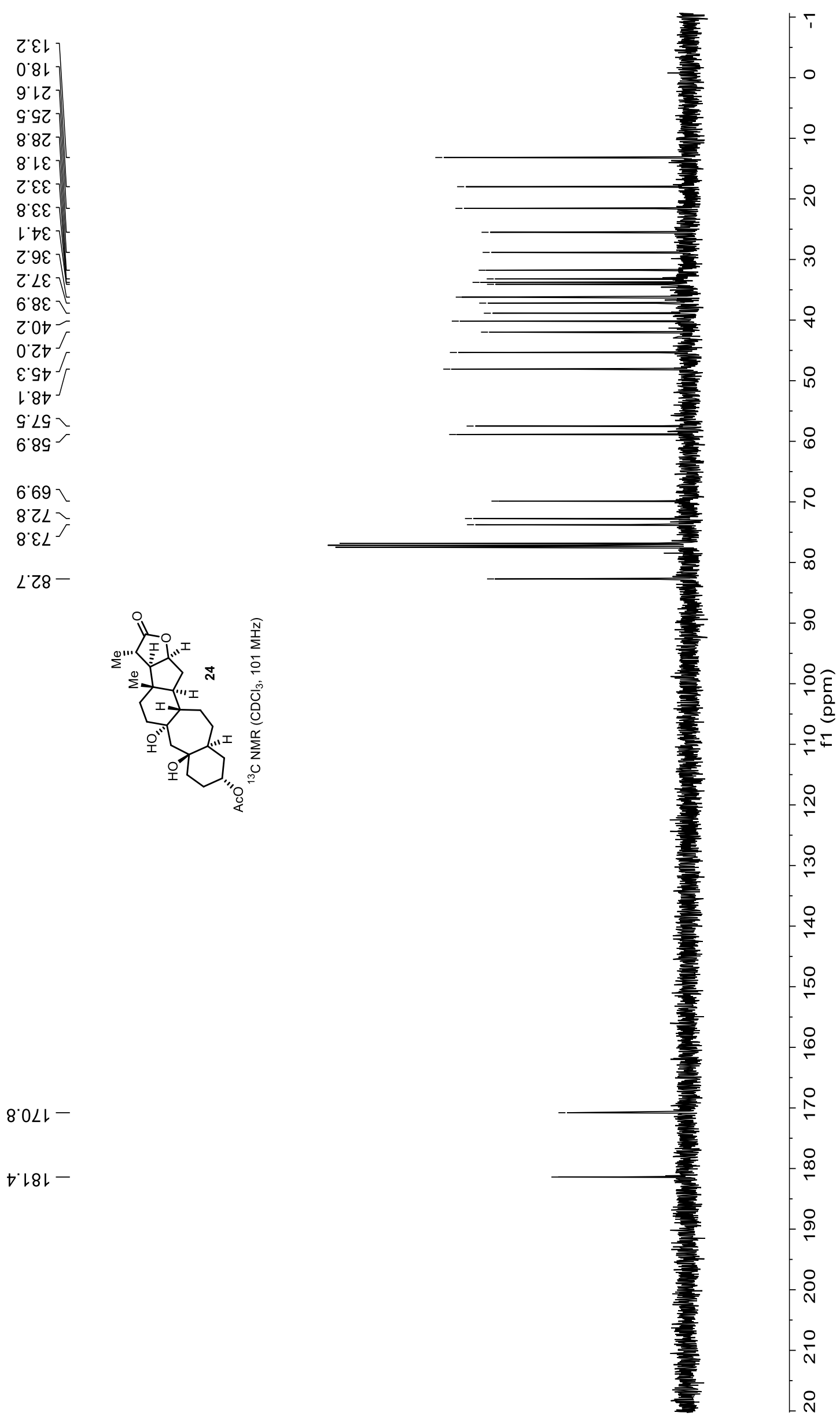
$\angle L^{\circ}-$

$\varepsilon 9^{\circ} 0$

$59^{\circ} 0$

$\angle 9^{\circ} 0$

$69^{\circ} 0$

$\angle L O O$

$\varepsilon L^{\circ} 0$

$\angle L^{\circ} O$

$\angle 6^{\circ} 0^{\circ}$

$66^{\circ} 0$

เ०

$0 \varepsilon^{\prime}\llcorner$

乙E'

GE'

$67^{\circ}$

IG'

$89^{\circ} \mathrm{L}$

†L L

$8 L$ L

$78^{\circ}$

$98^{\circ} \mathrm{L}$

96 .

$\varepsilon 0^{\circ} 乙$

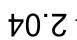

จL'

$\nabla z \cdot z$

$92 \cdot$

०६ $乙$

$\varepsilon \mathcal{G}^{\prime} 乙$

SG'

$\angle S^{\circ} Z$

$69^{\circ} \mathrm{Z}$

$26 . \nabla$

$\varepsilon \sigma^{\circ} \nabla$

$\nabla 6 \circ$

$96 \circ$

$96 . t$

$\angle 6^{\circ} \circ$

$00 \mathrm{~s}$

10.9

20.9

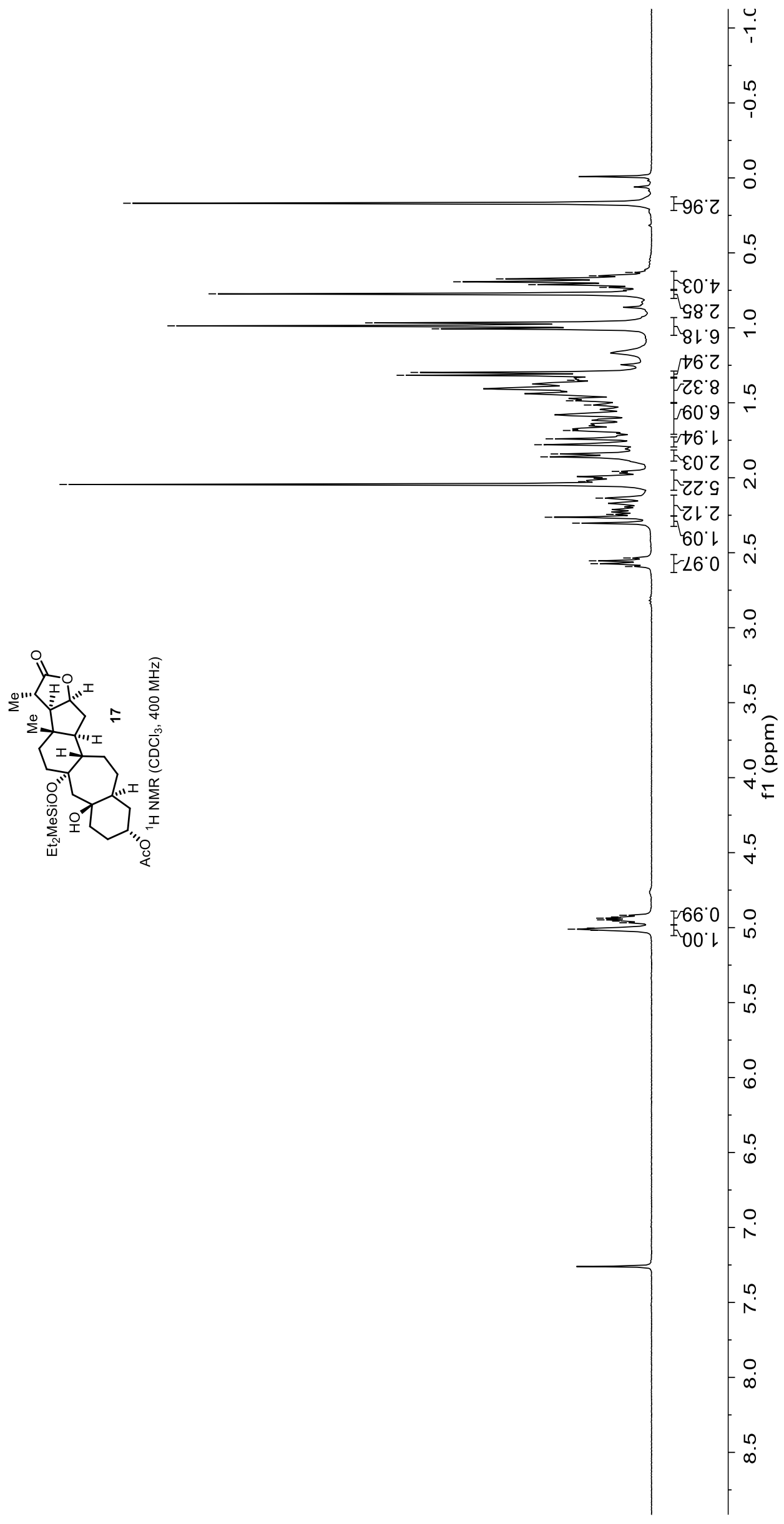




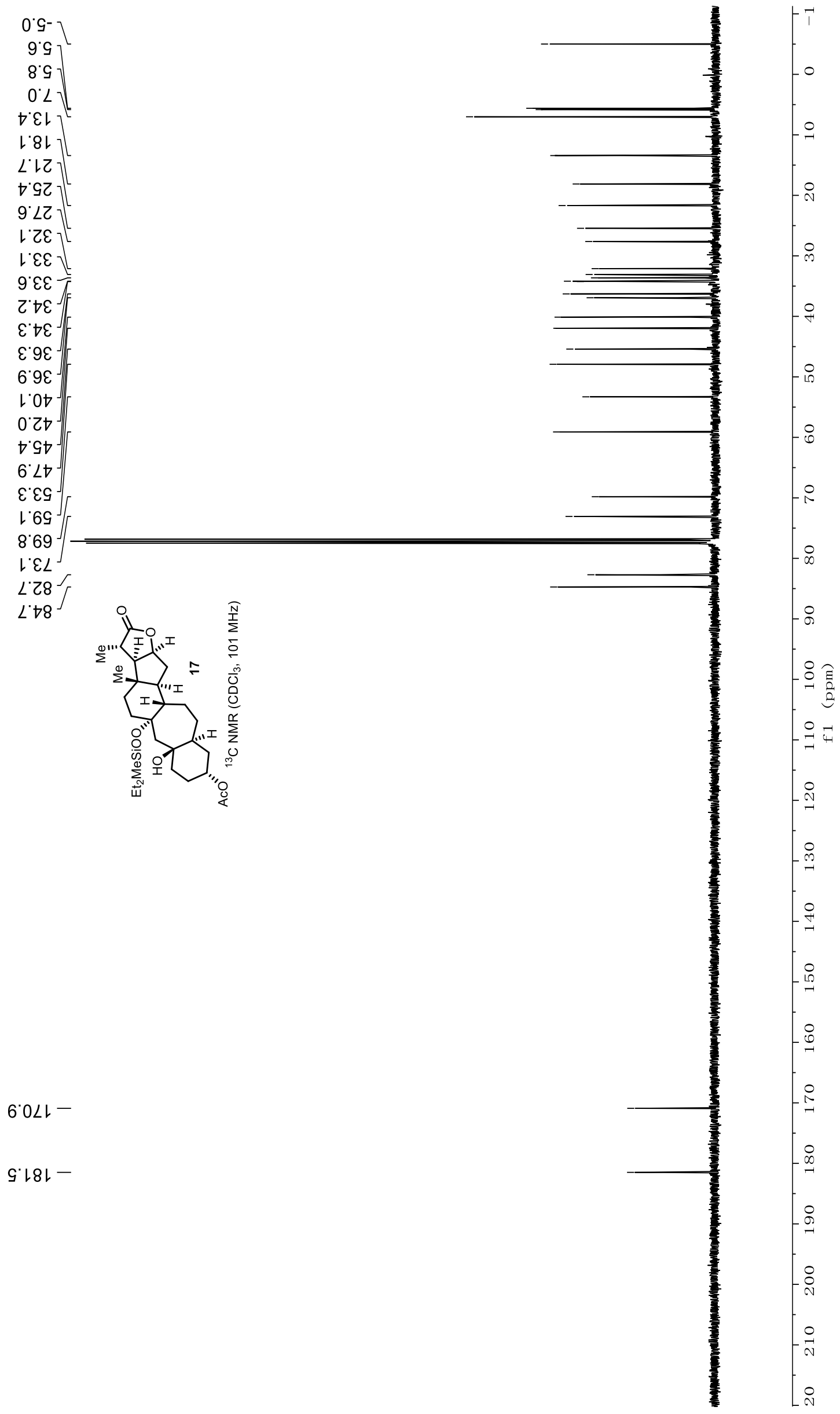




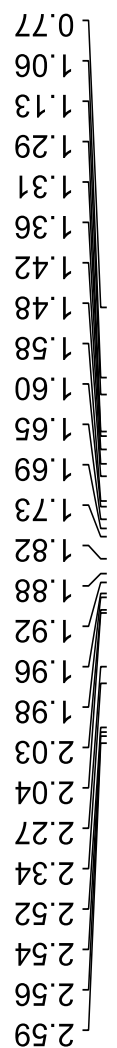

$\varepsilon 6^{\circ} t$

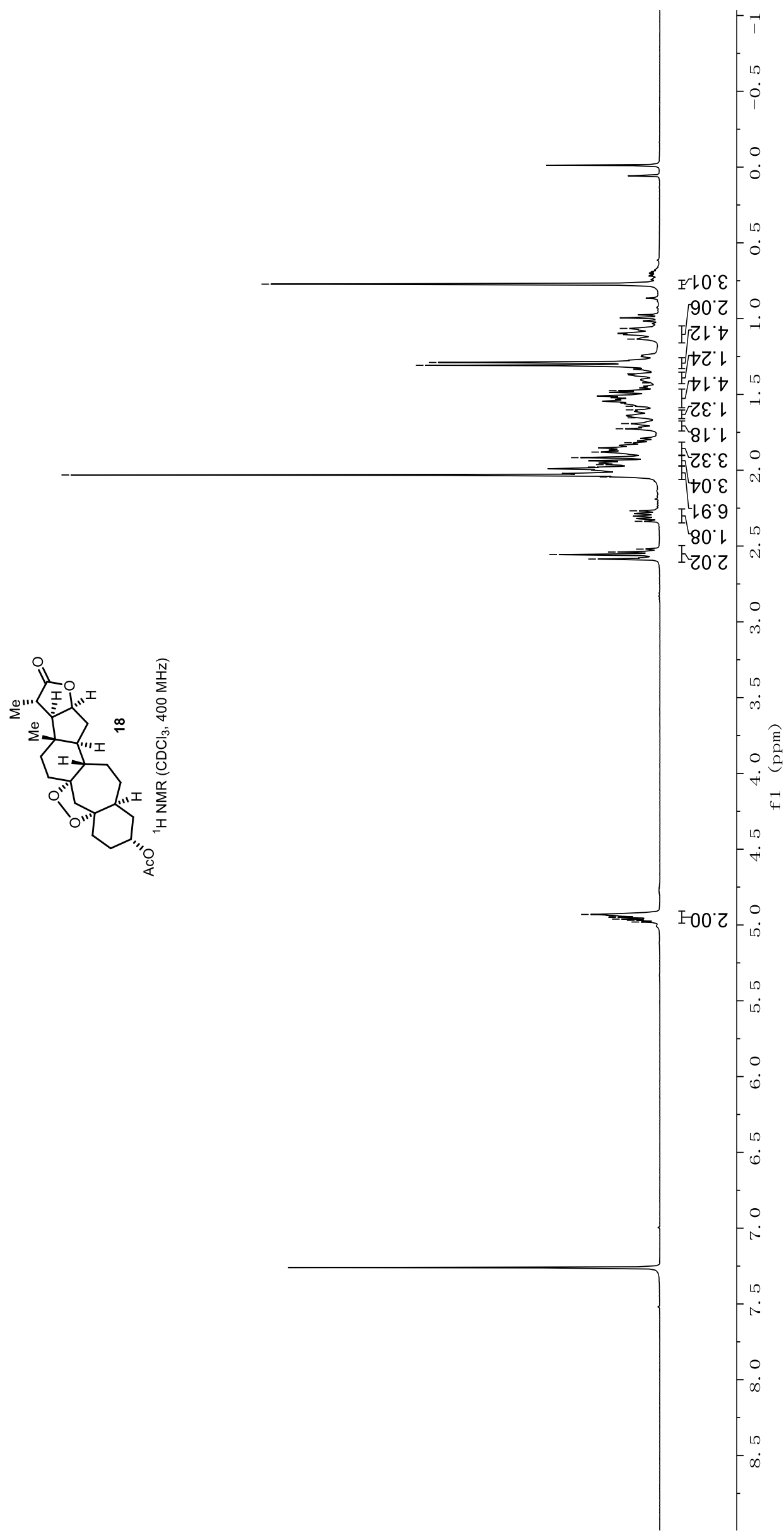



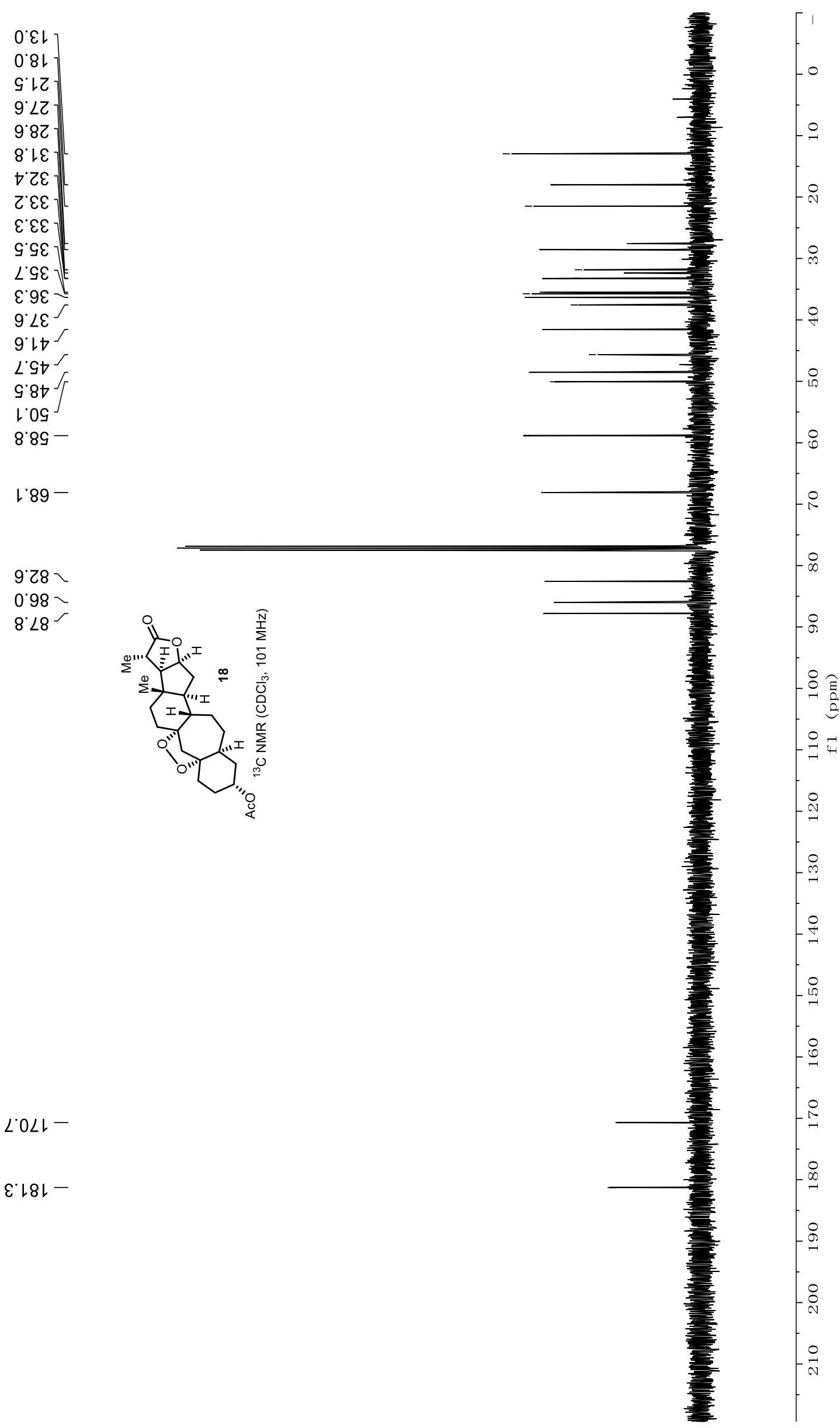


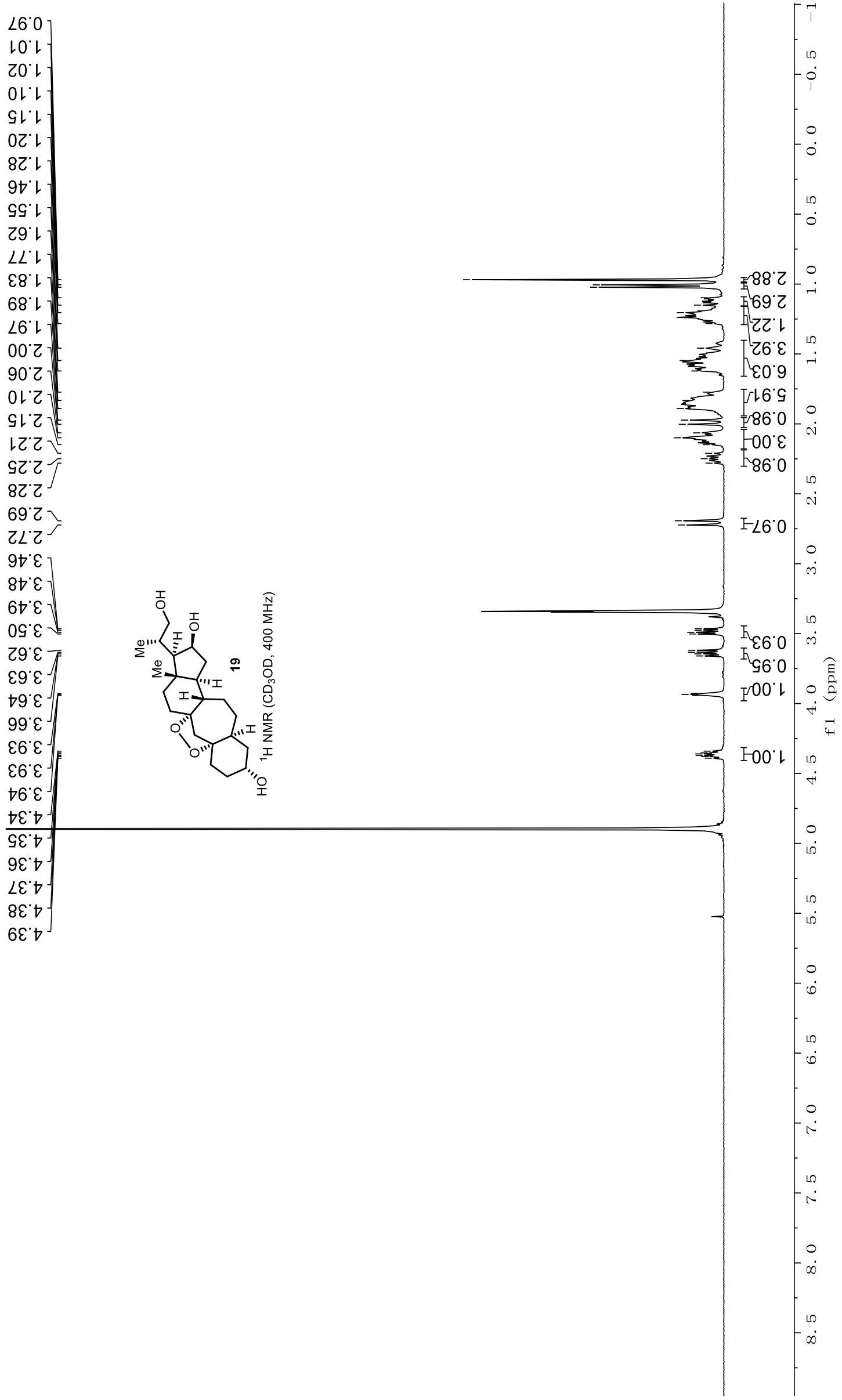




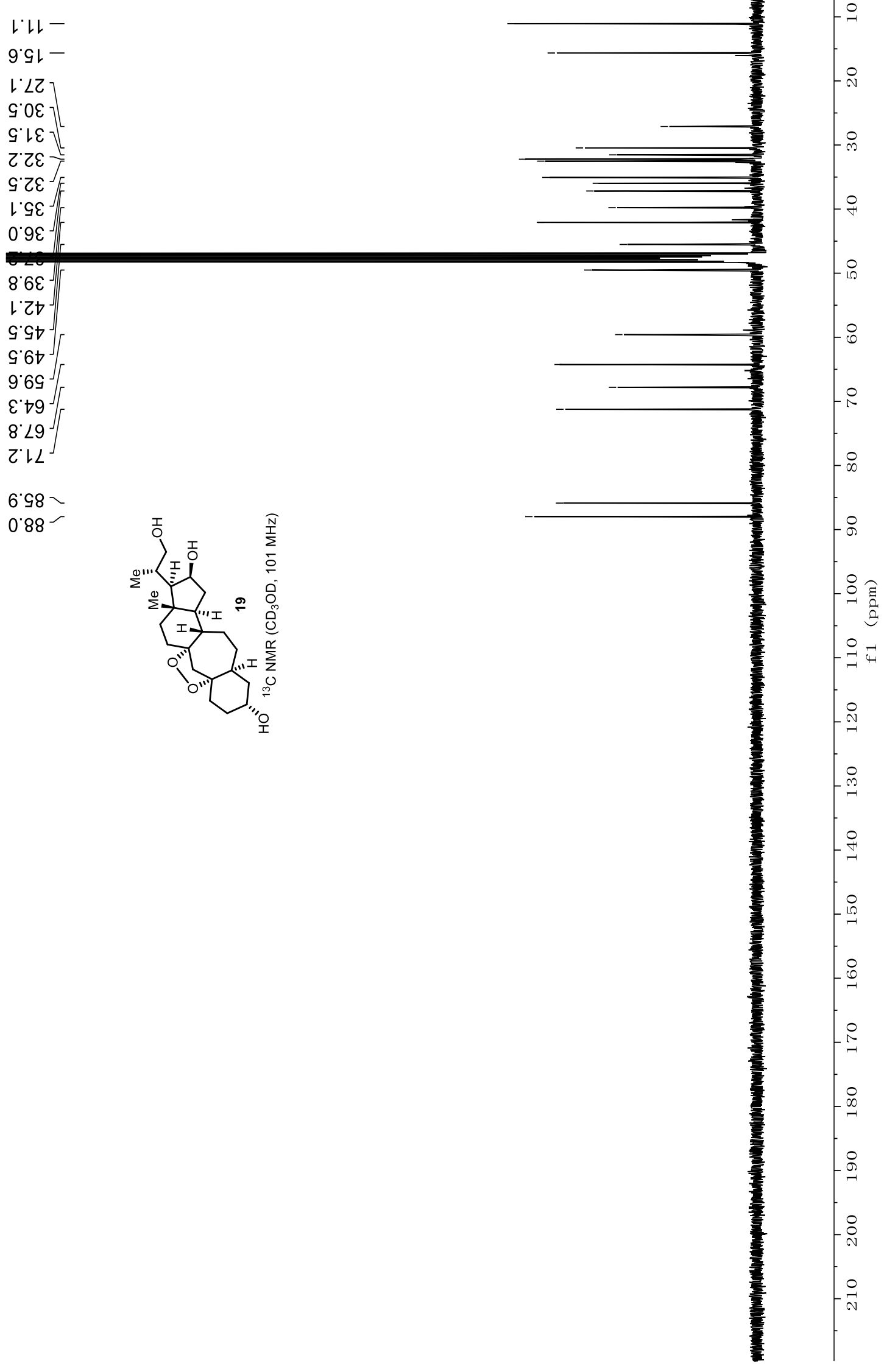



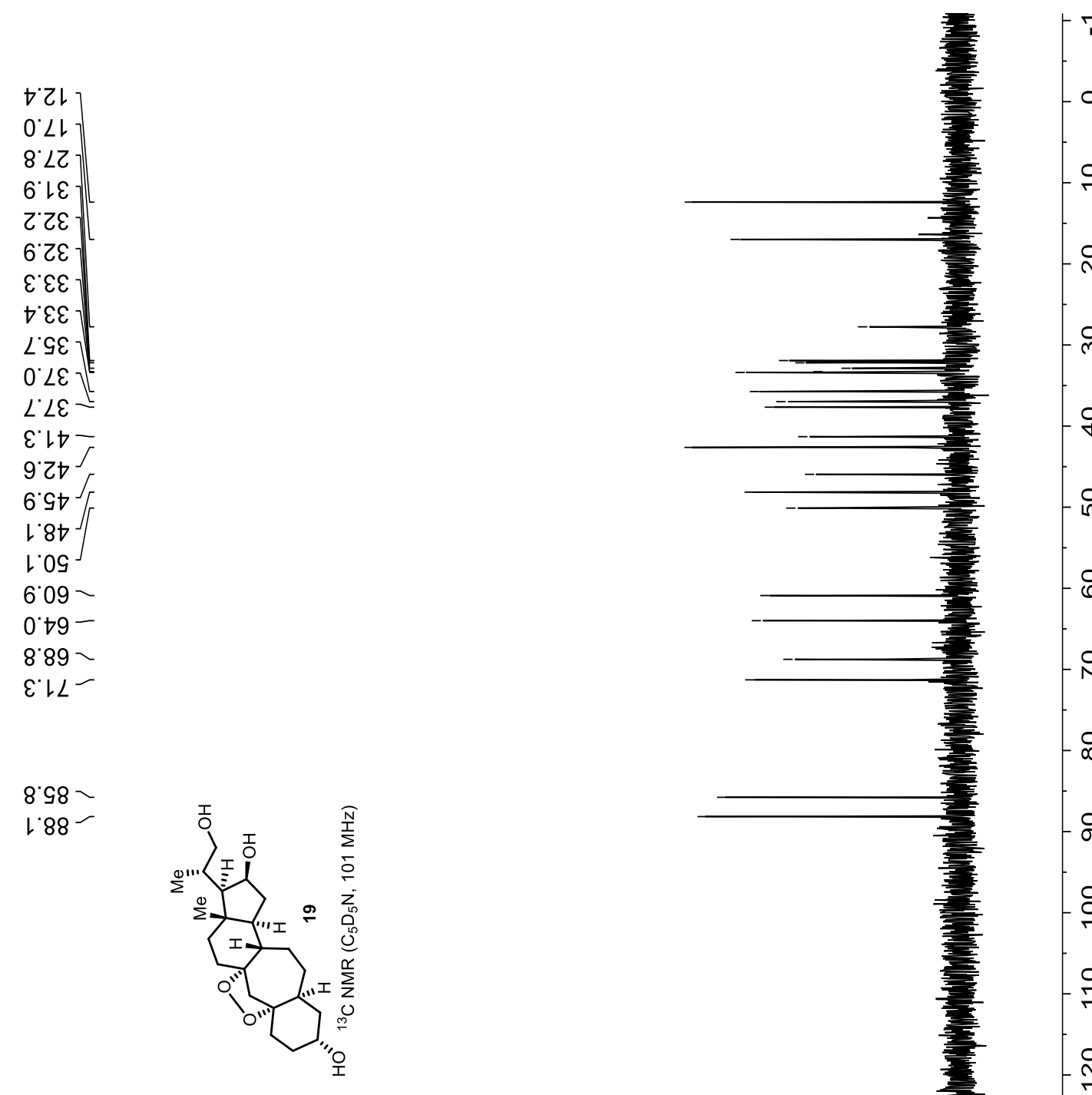

$\infty$

$\circ$

은

아

ㅇ

옴

$\stackrel{ }{\leftarrow}$

iᄋ

$\stackrel{8}{\circ}$

$\stackrel{ }{ }$

$\infty$

욤

ํํ

ํำ 

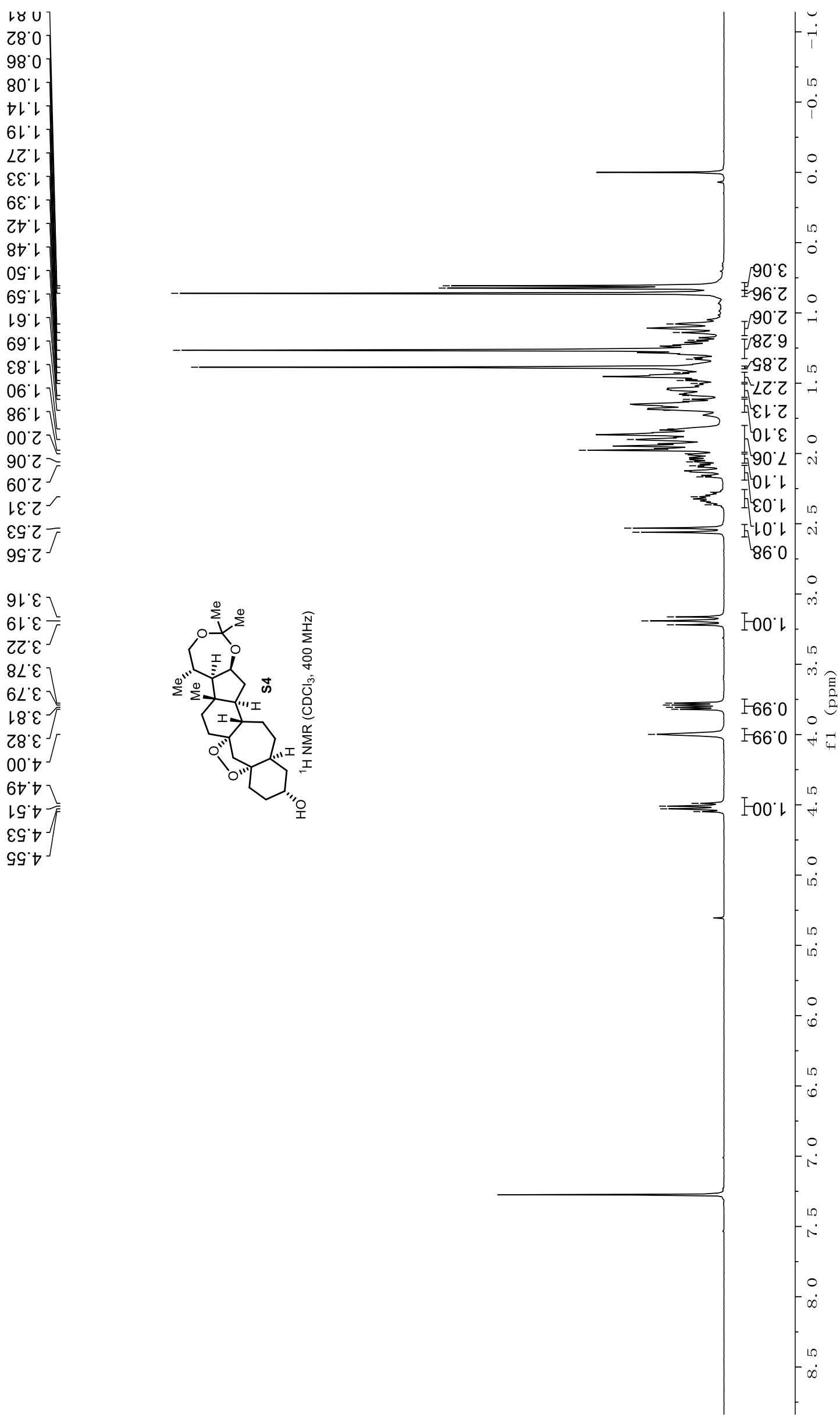

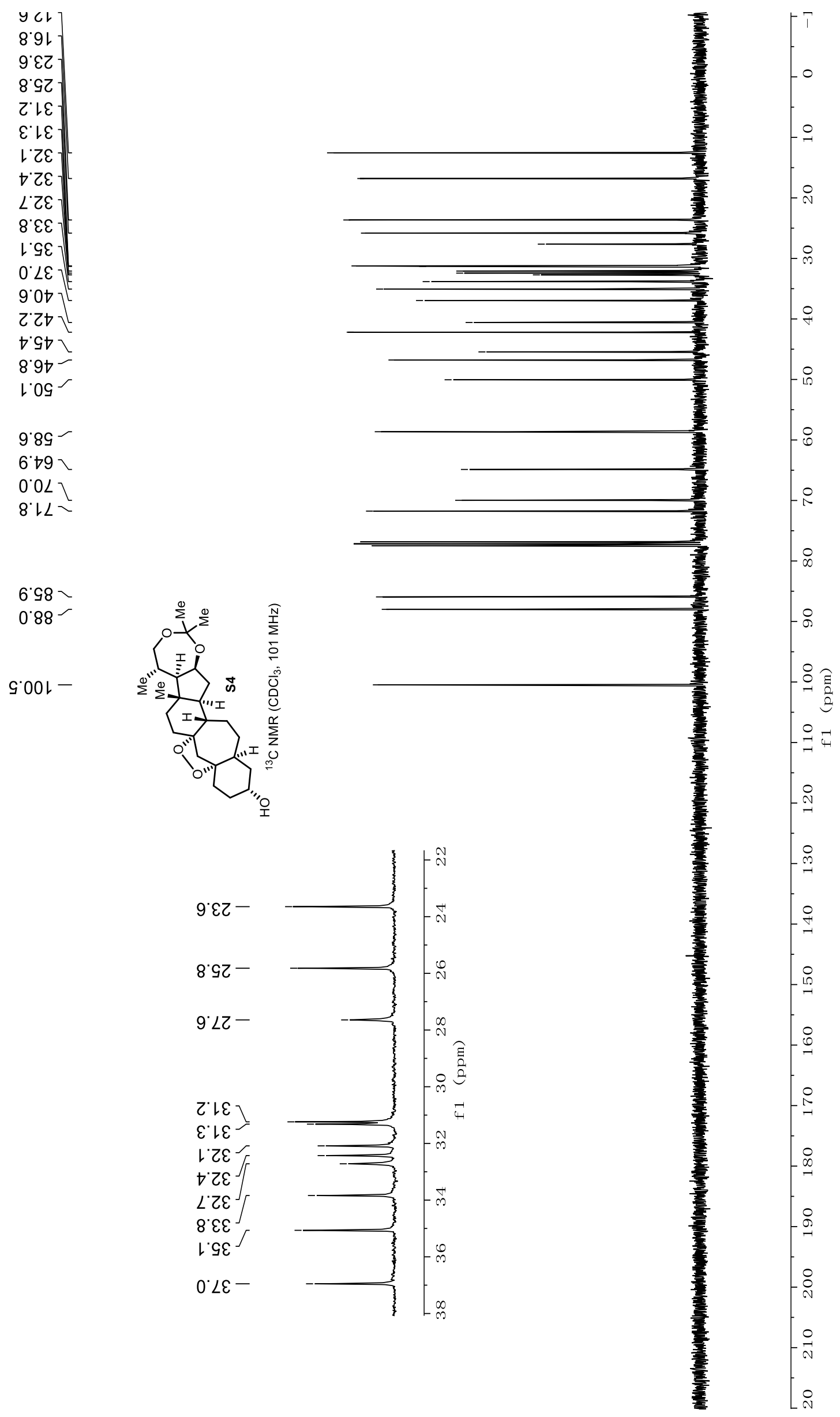

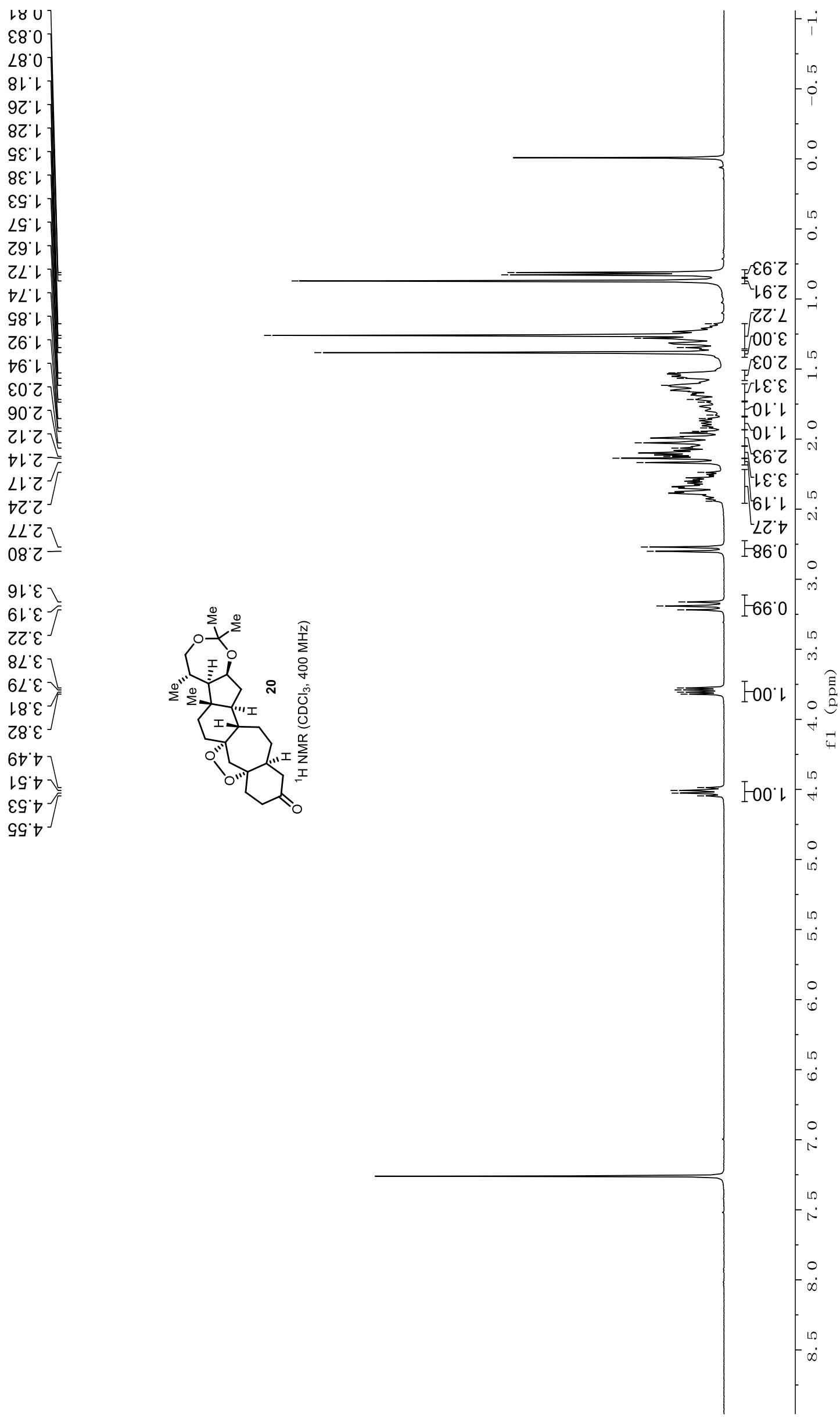


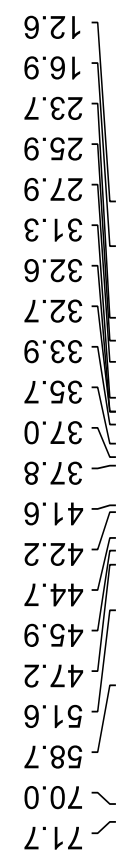

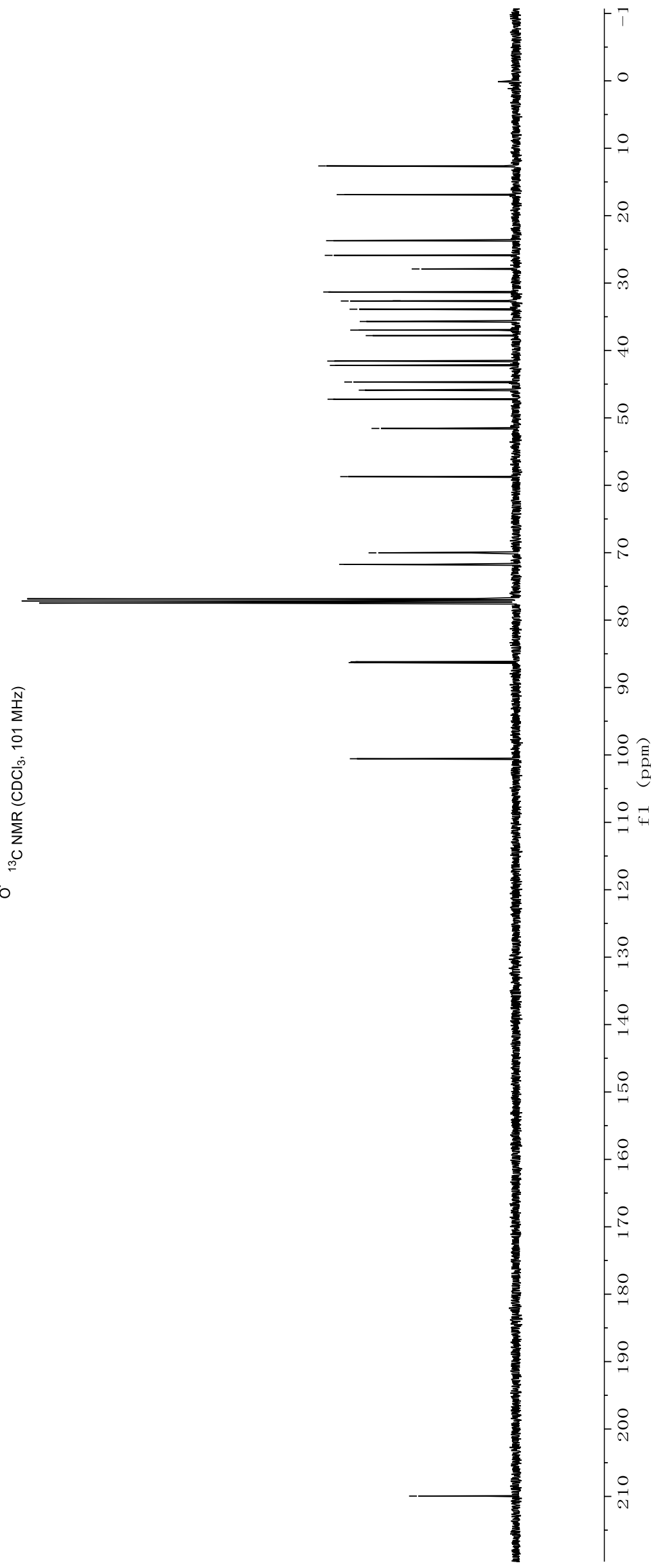

$\left.\begin{array}{l}\tau^{\prime} 98 \\ \varepsilon^{\prime} 98\end{array}\right\}$

$9.001-$ 

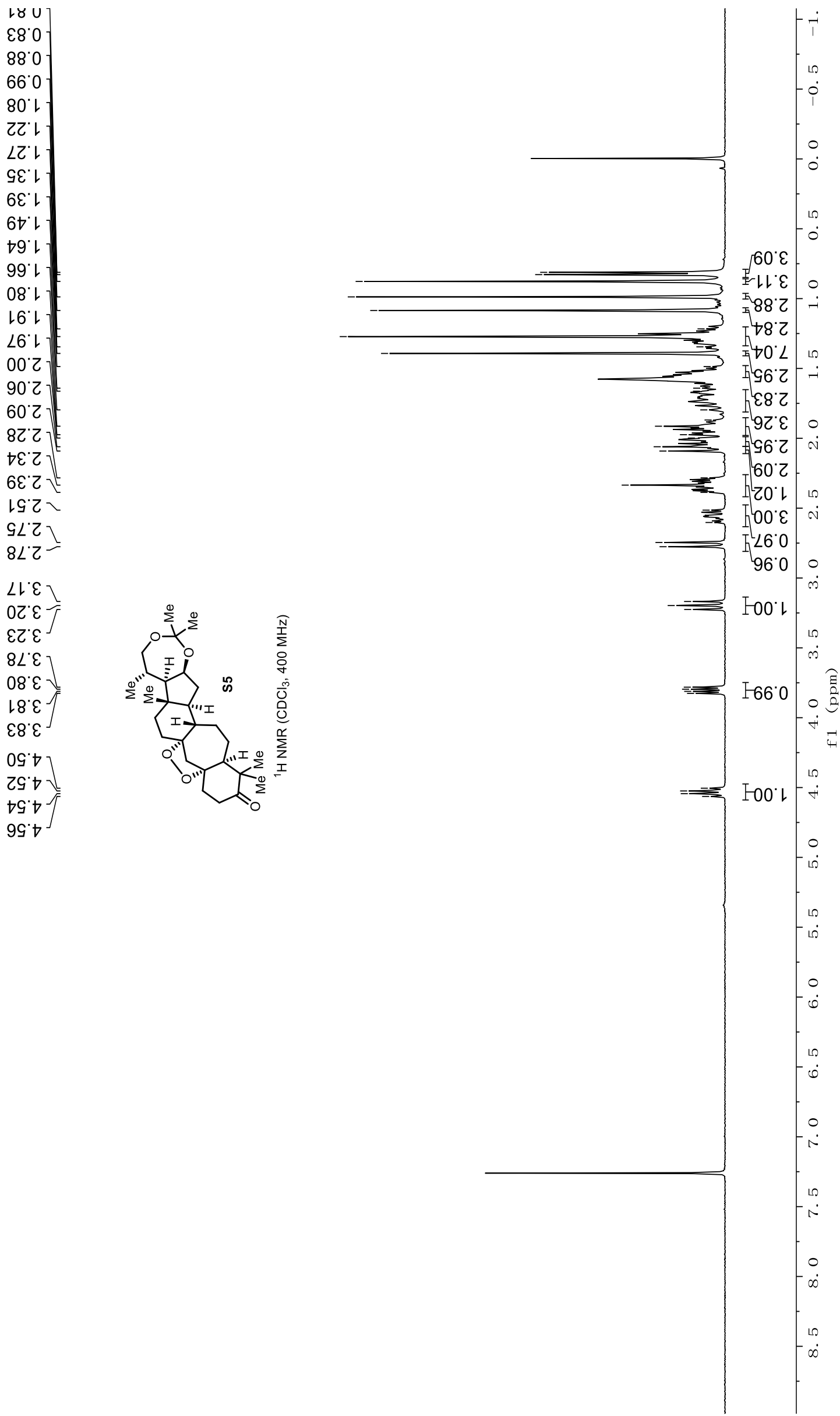


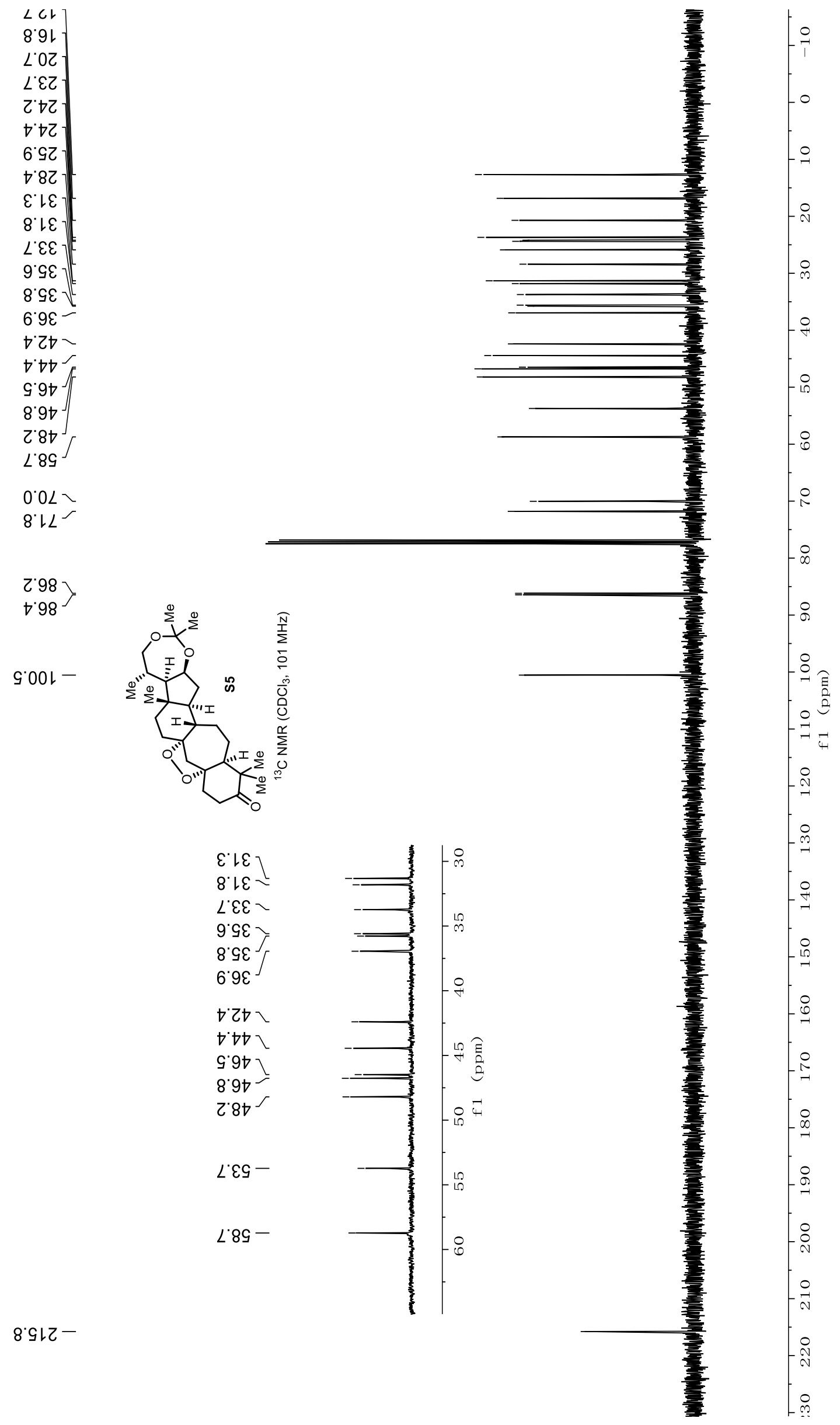



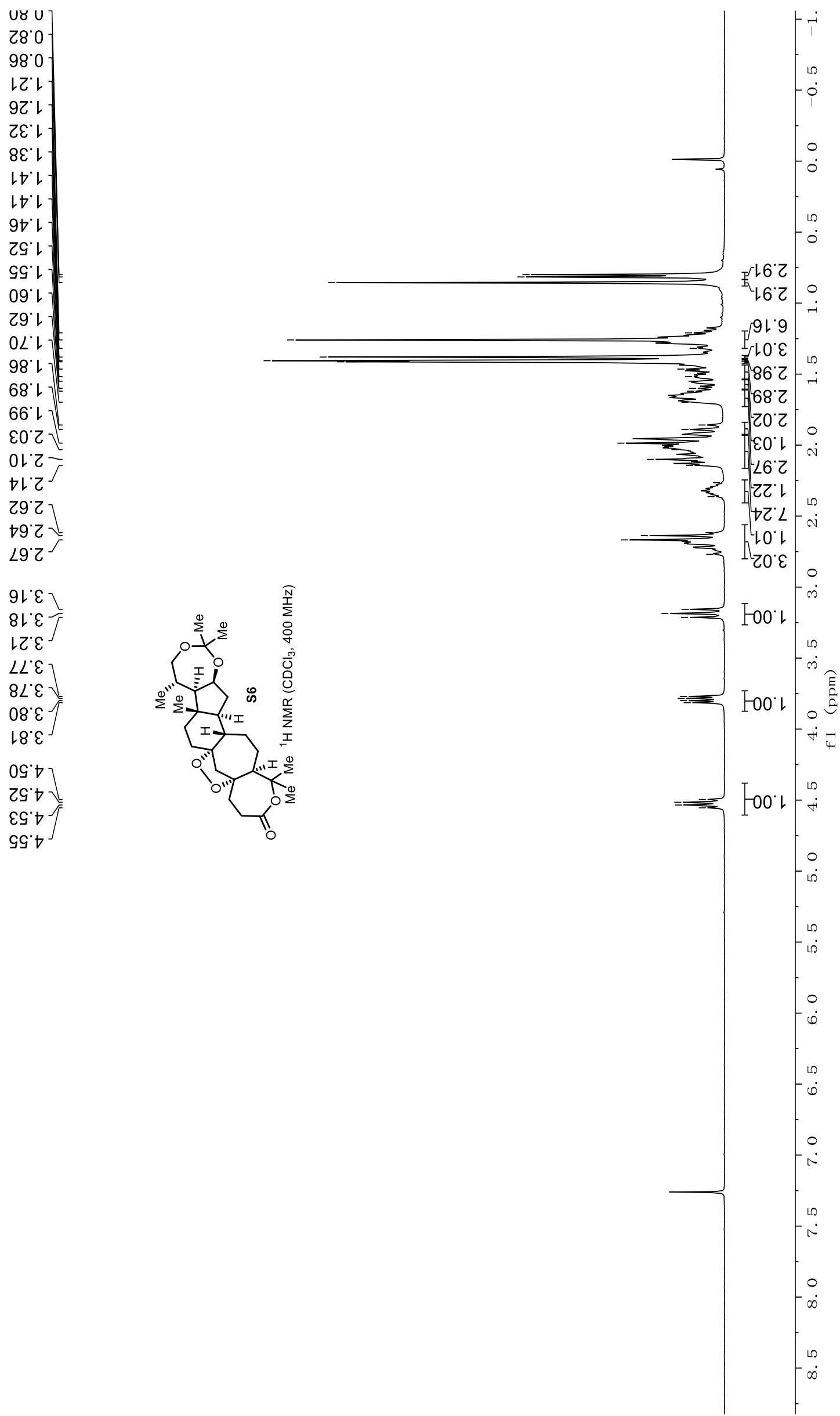


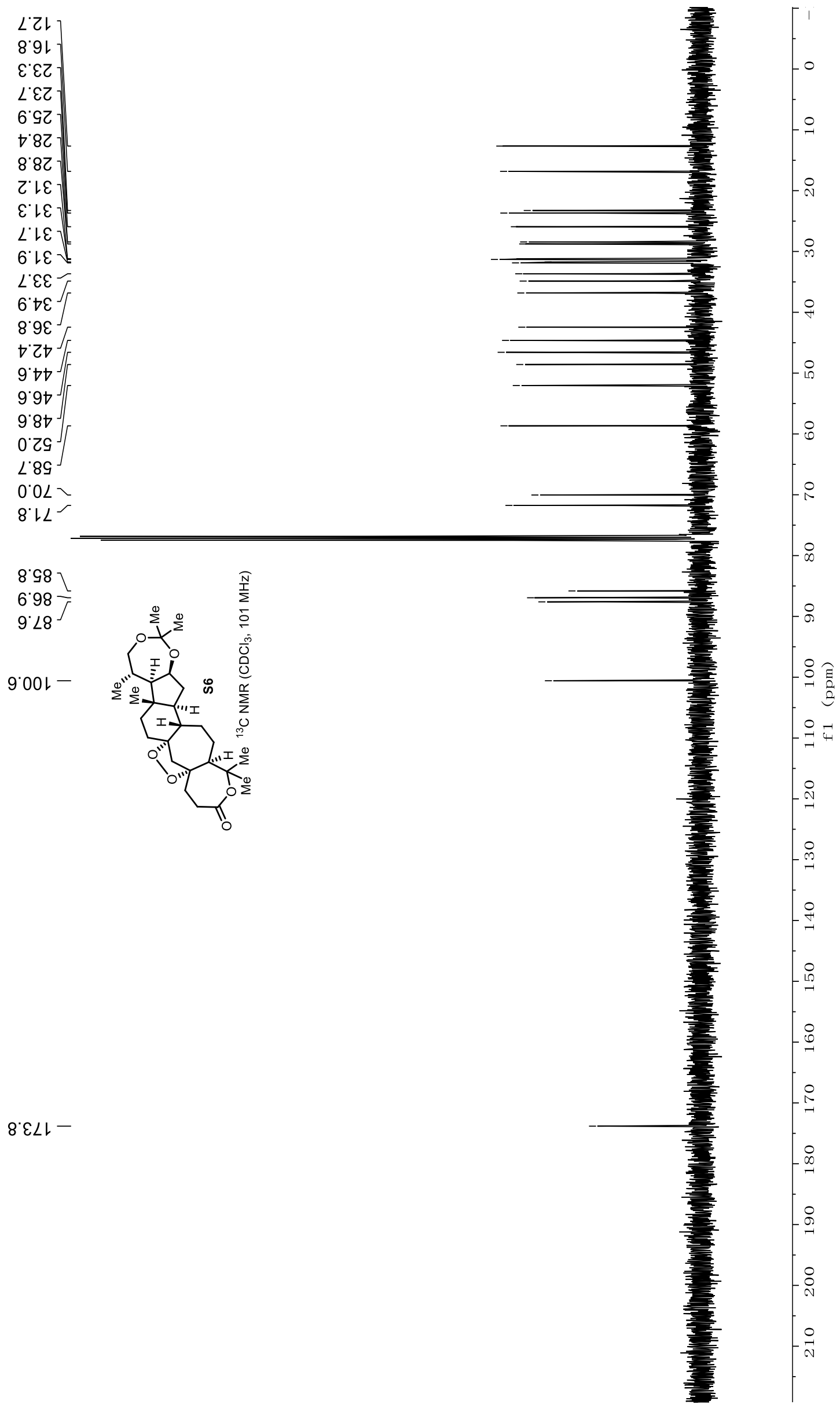



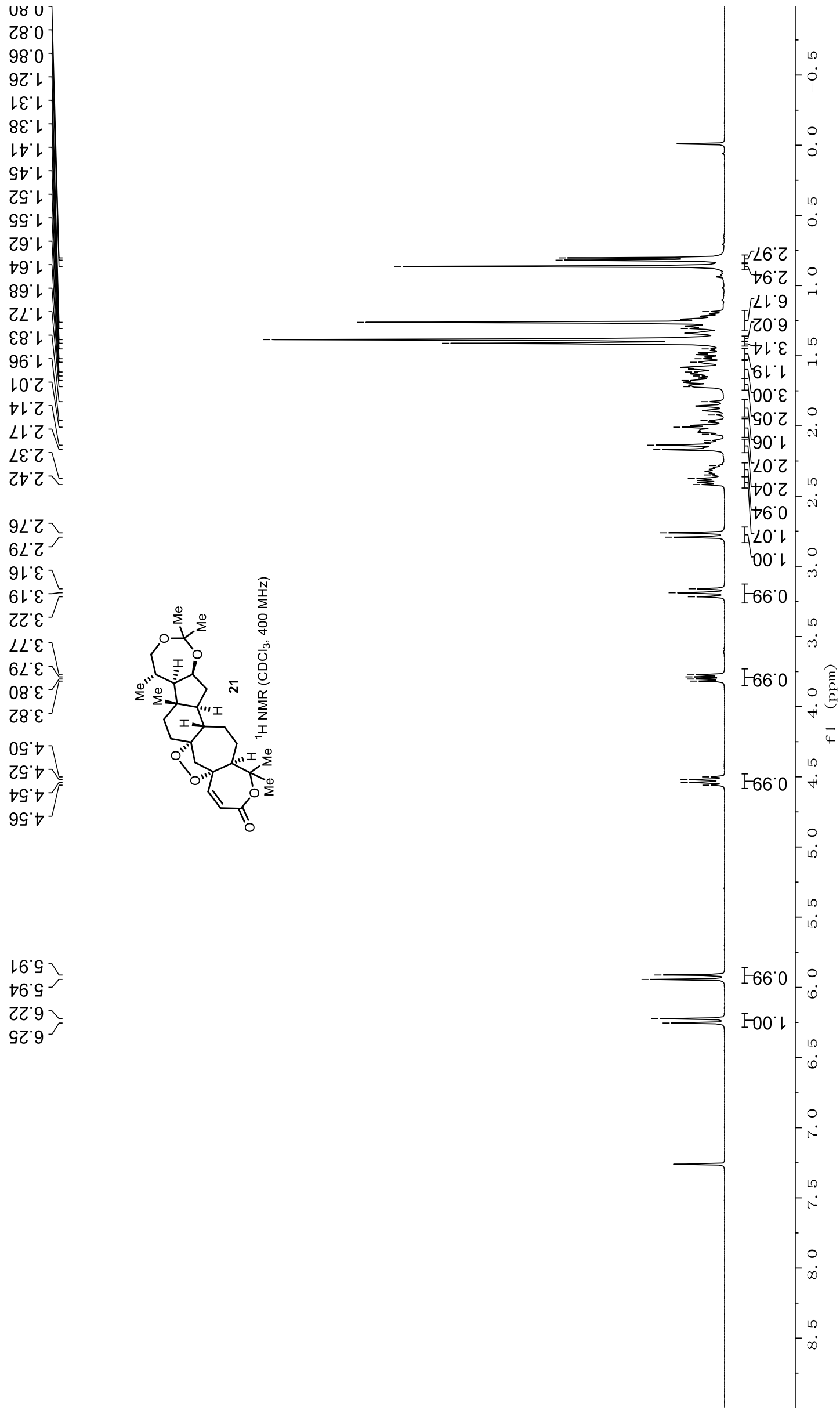

16.9

$\nabla 6^{\circ} \mathrm{s}^{\prime}$

टर 9 工

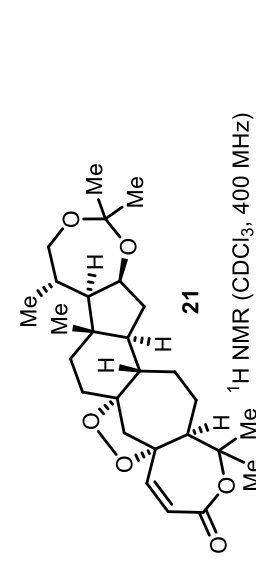

ง 9 


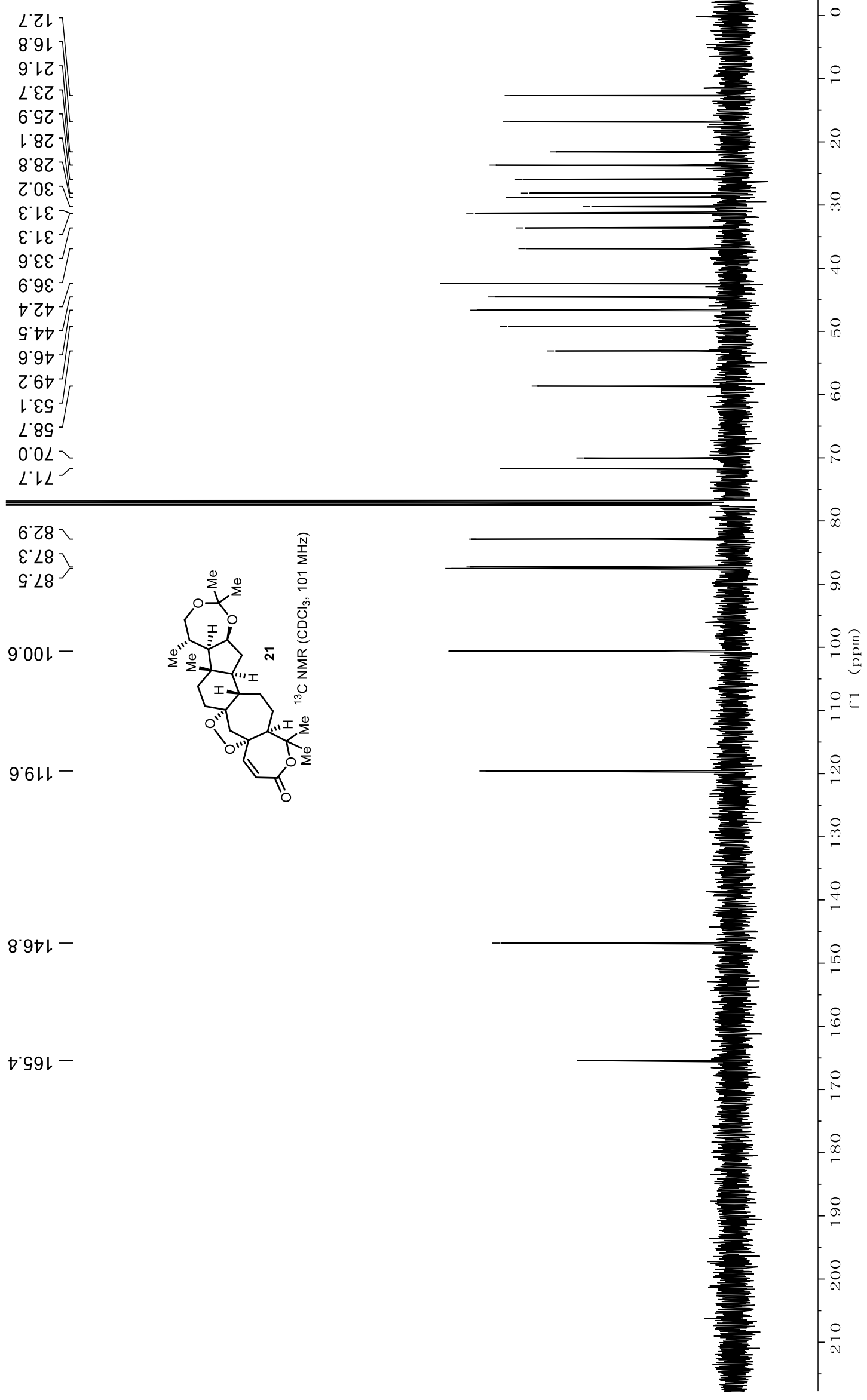




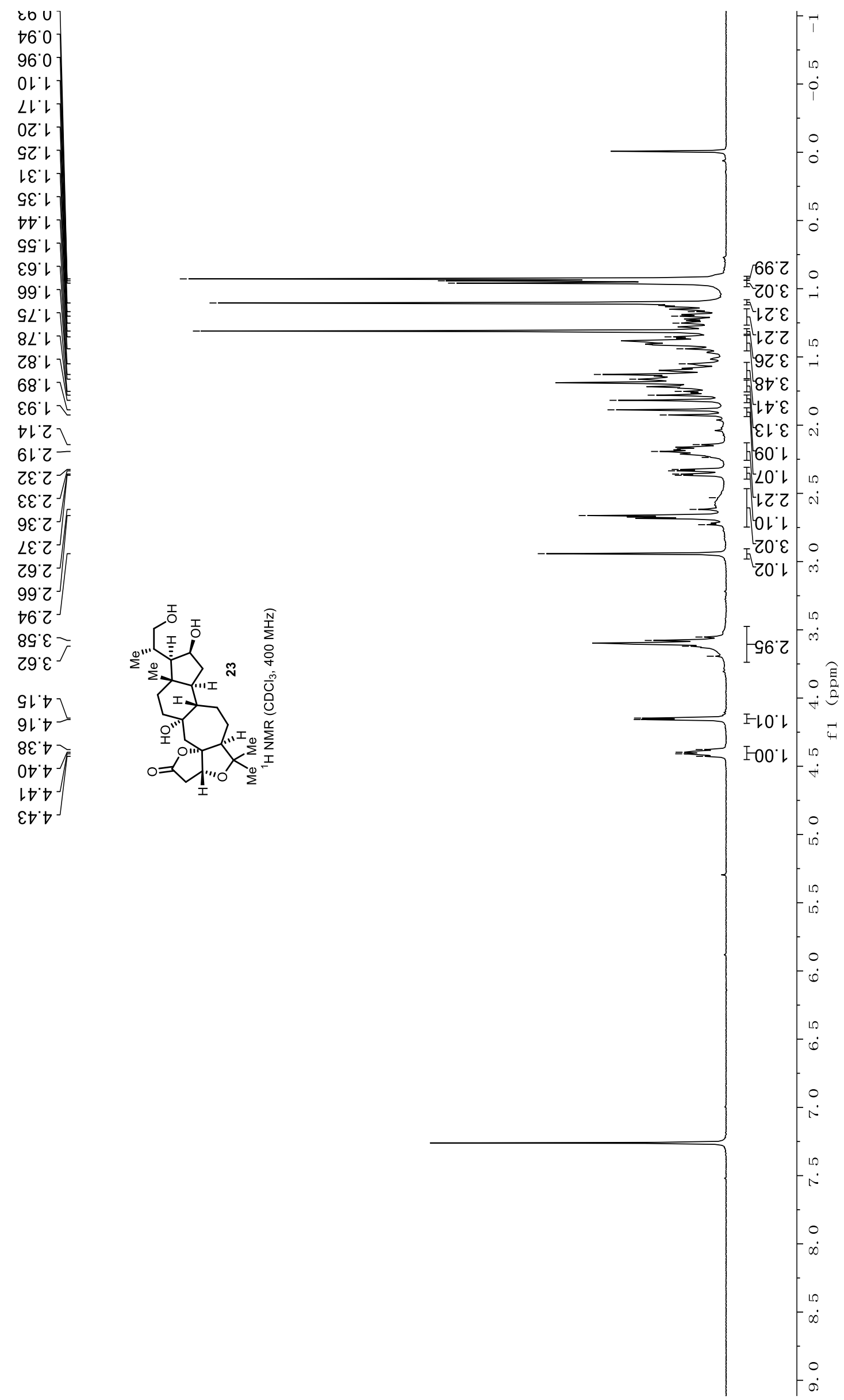




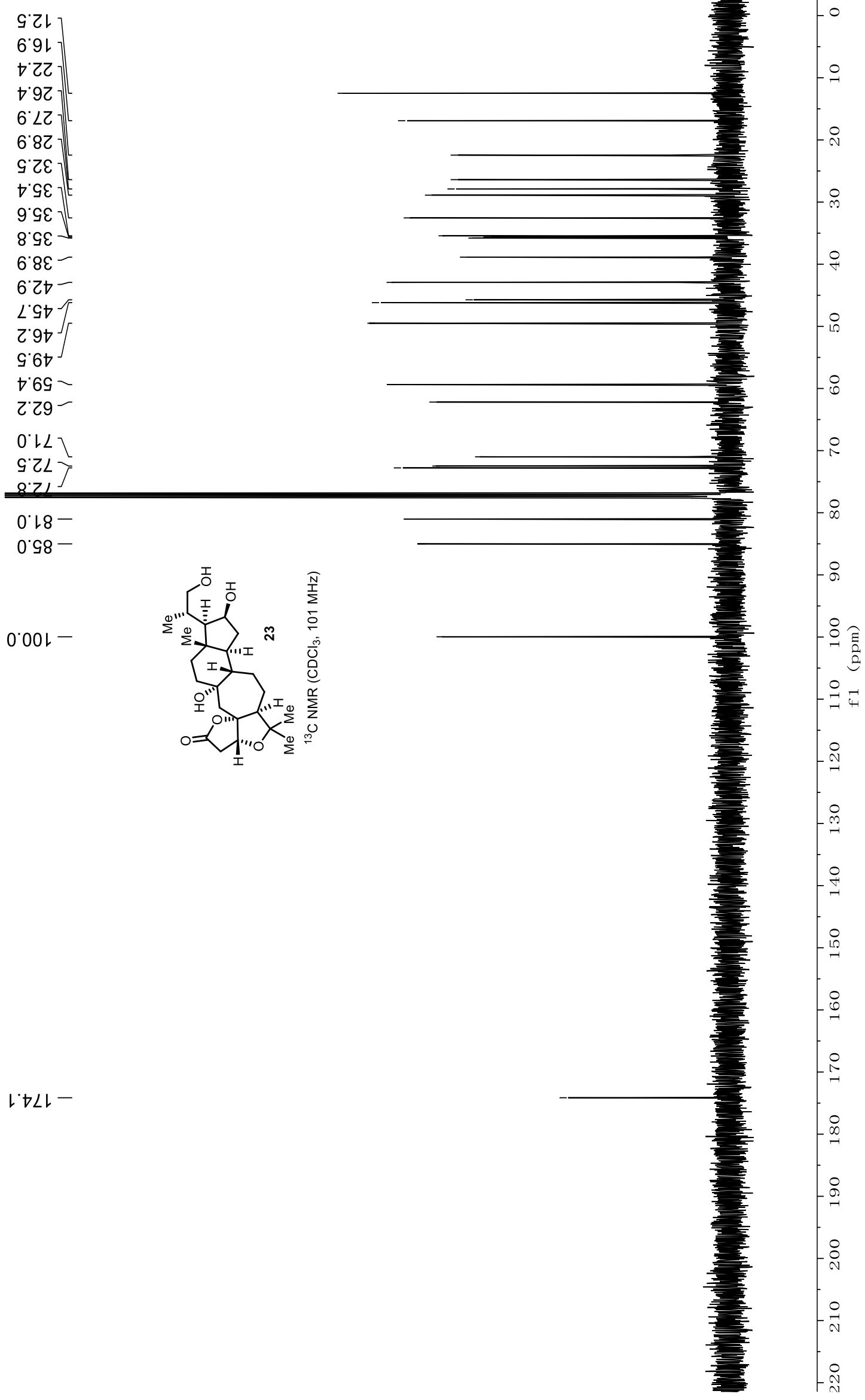



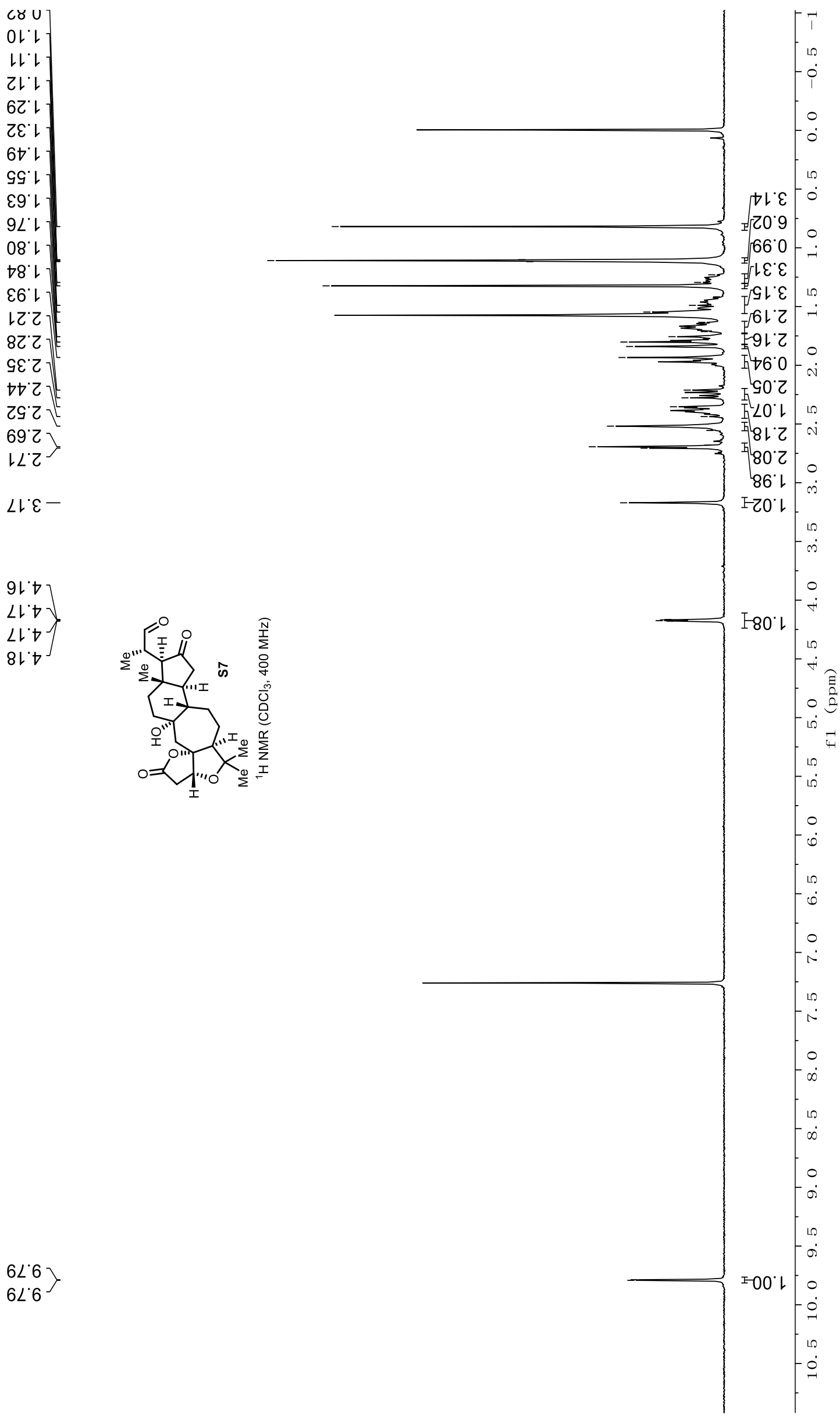

$6 L^{\circ} 6$

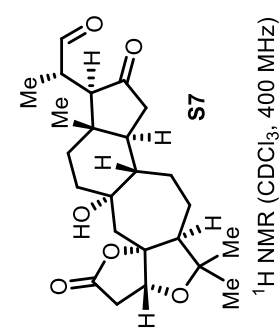

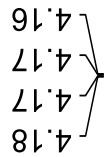

$6 L^{\circ} 6$ 


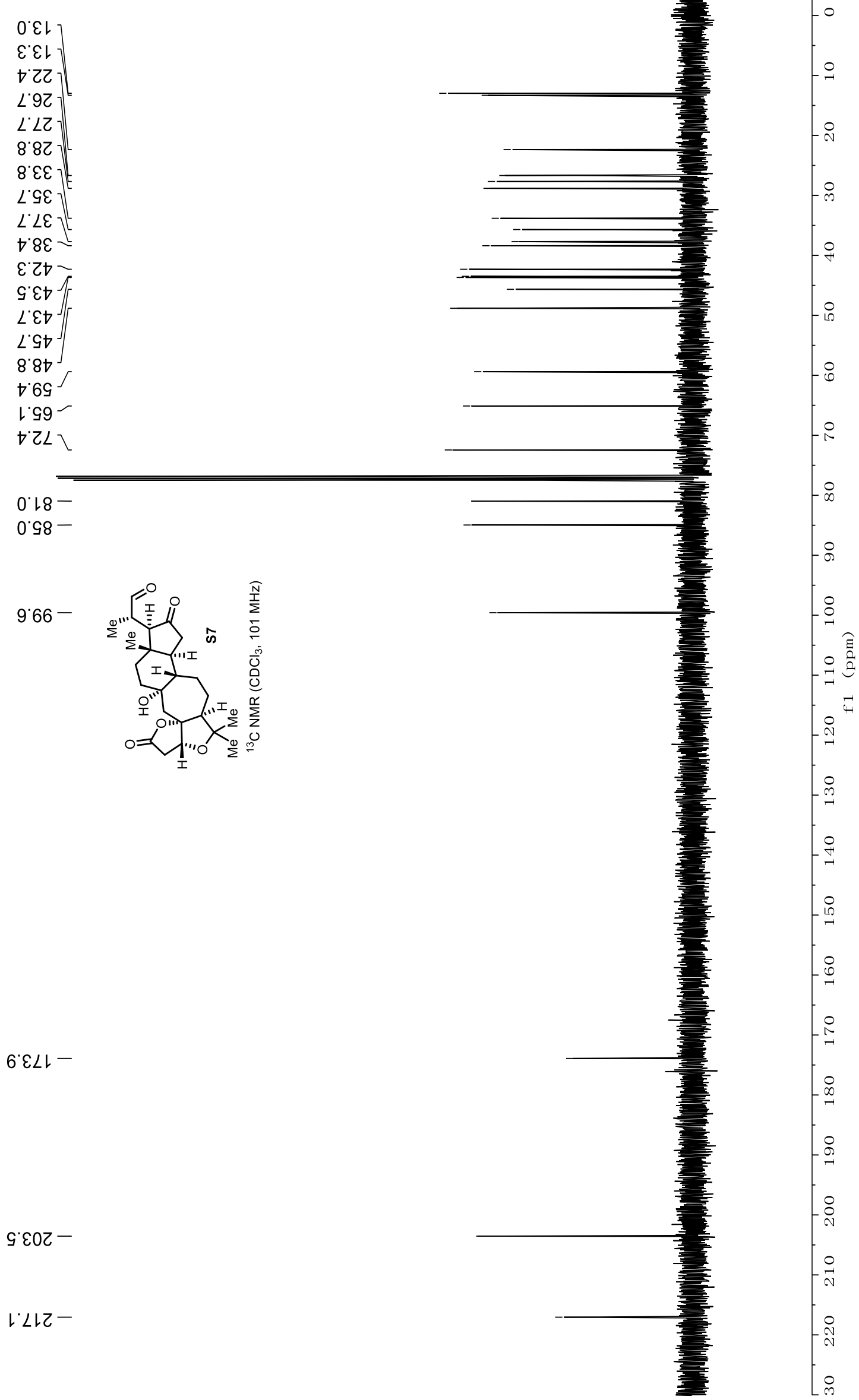




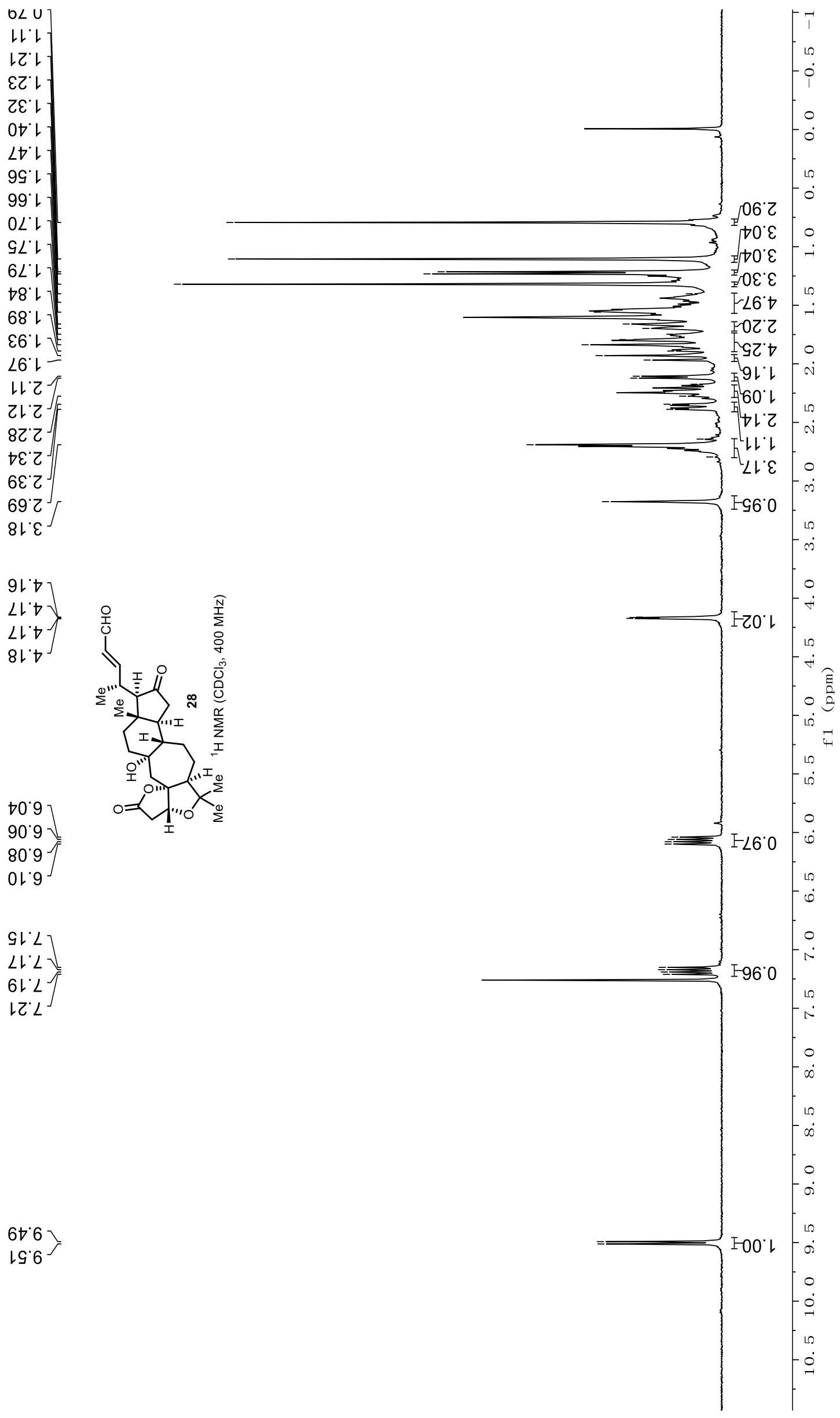




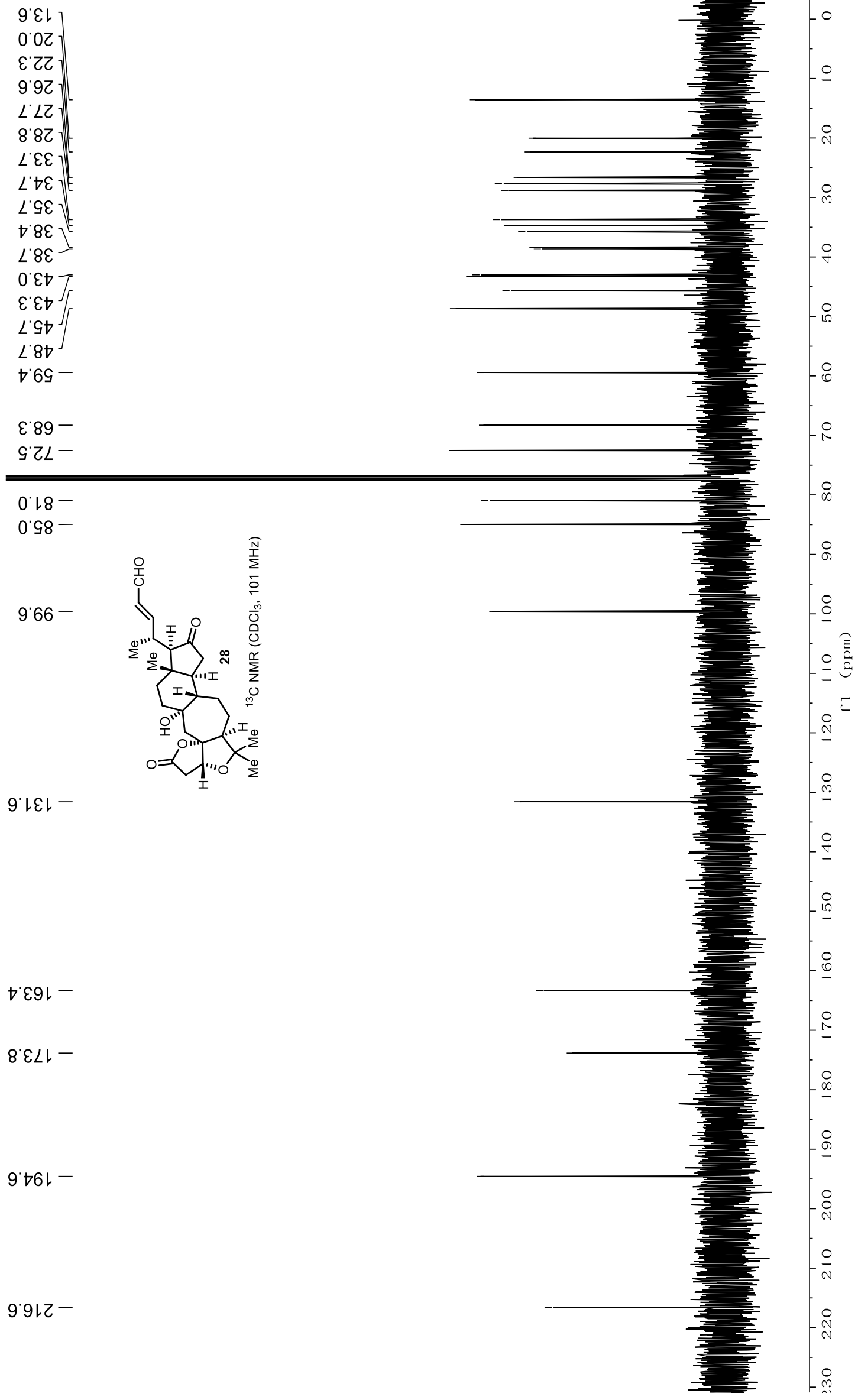




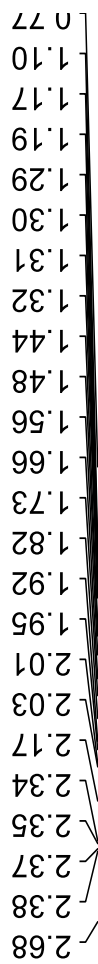

ol $\varepsilon-$
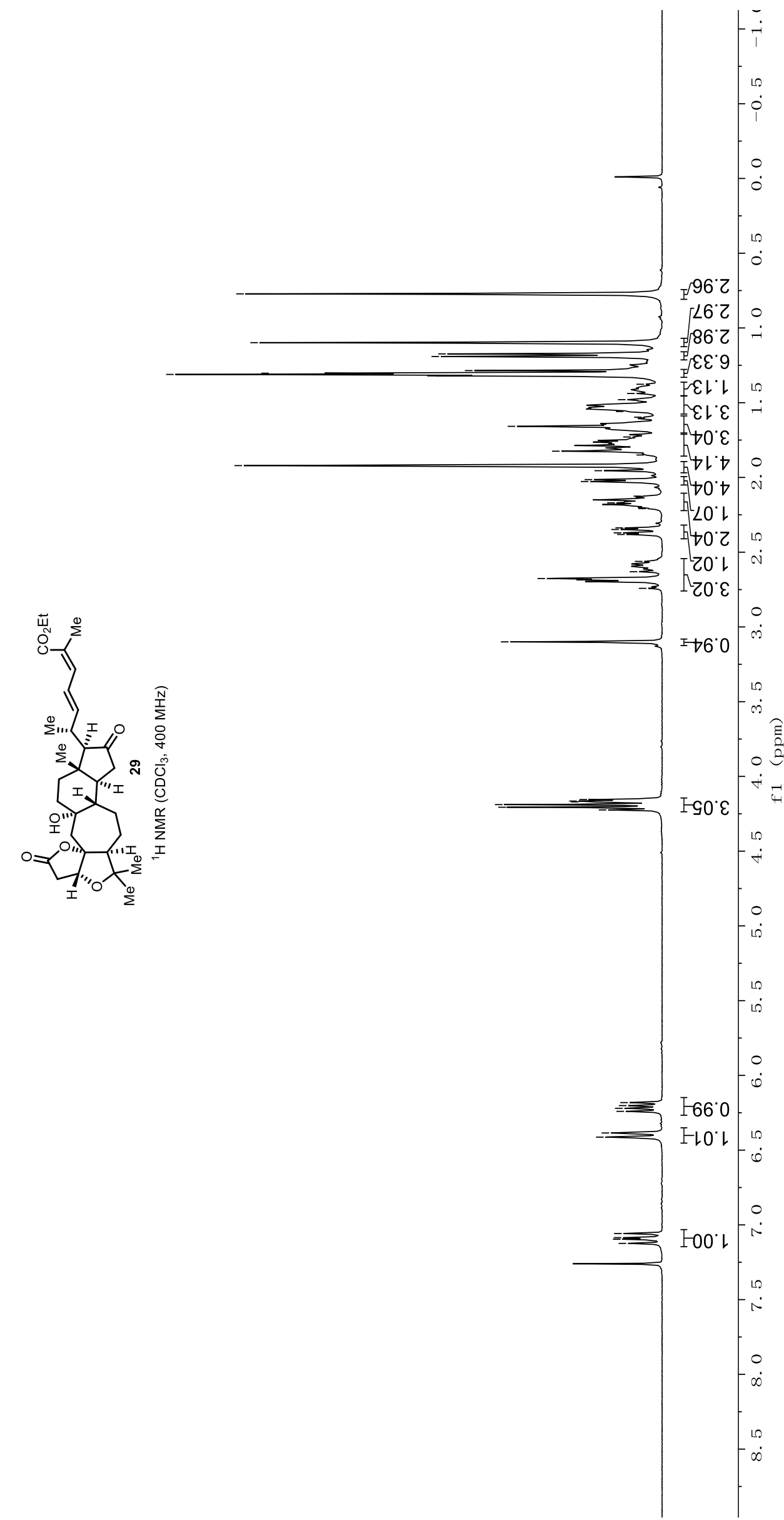

$81 \cdot 9$

$0 乙 \cdot 9$

乙乙'9

$\nabla Z \cdot 9$

$6 \varepsilon^{\prime} 9$

$เ \nabla \cdot 9$

$90^{\circ} \mathrm{L}$

$60^{\circ} \mathrm{L}$

$O L L$
CLL 


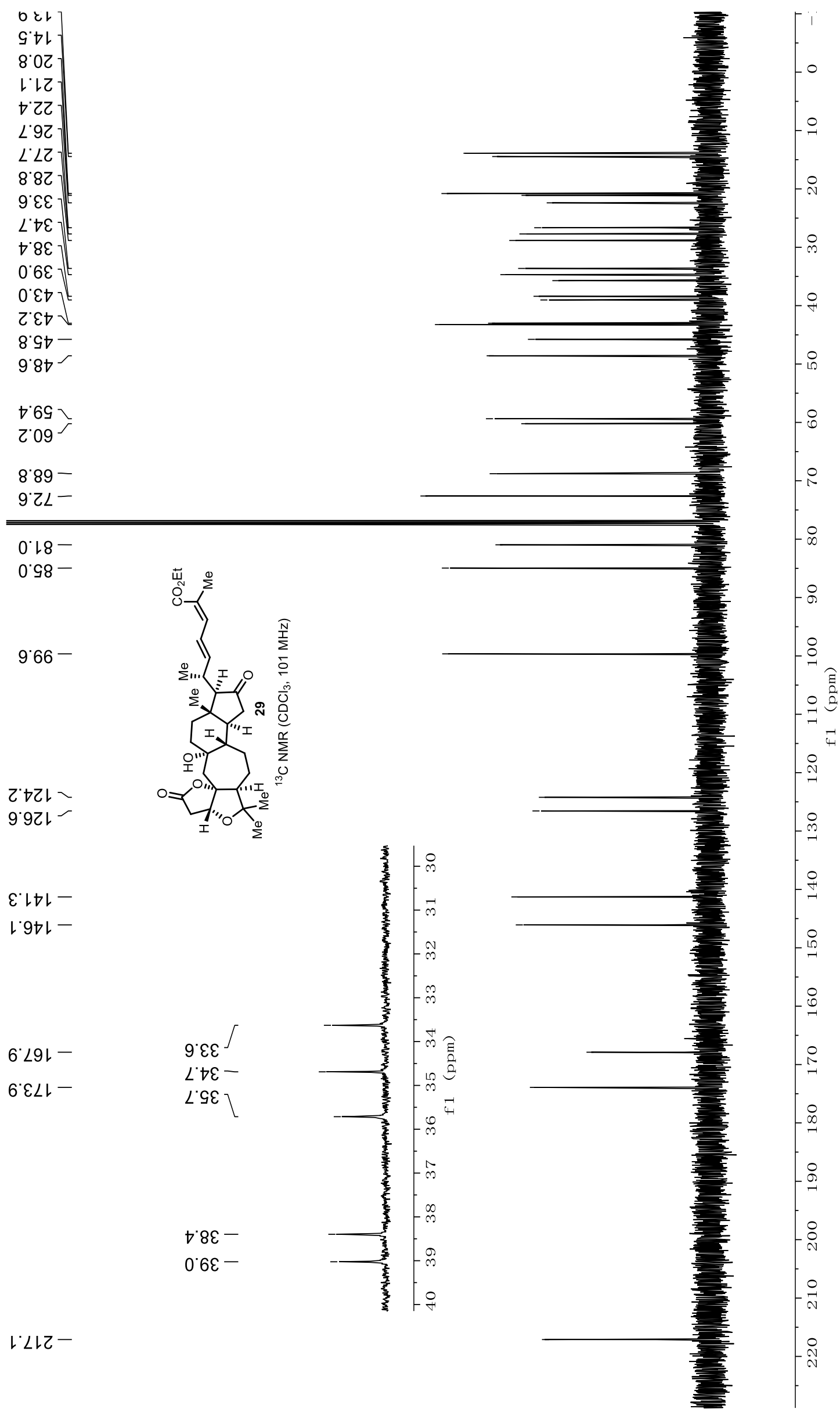


(wdd) If

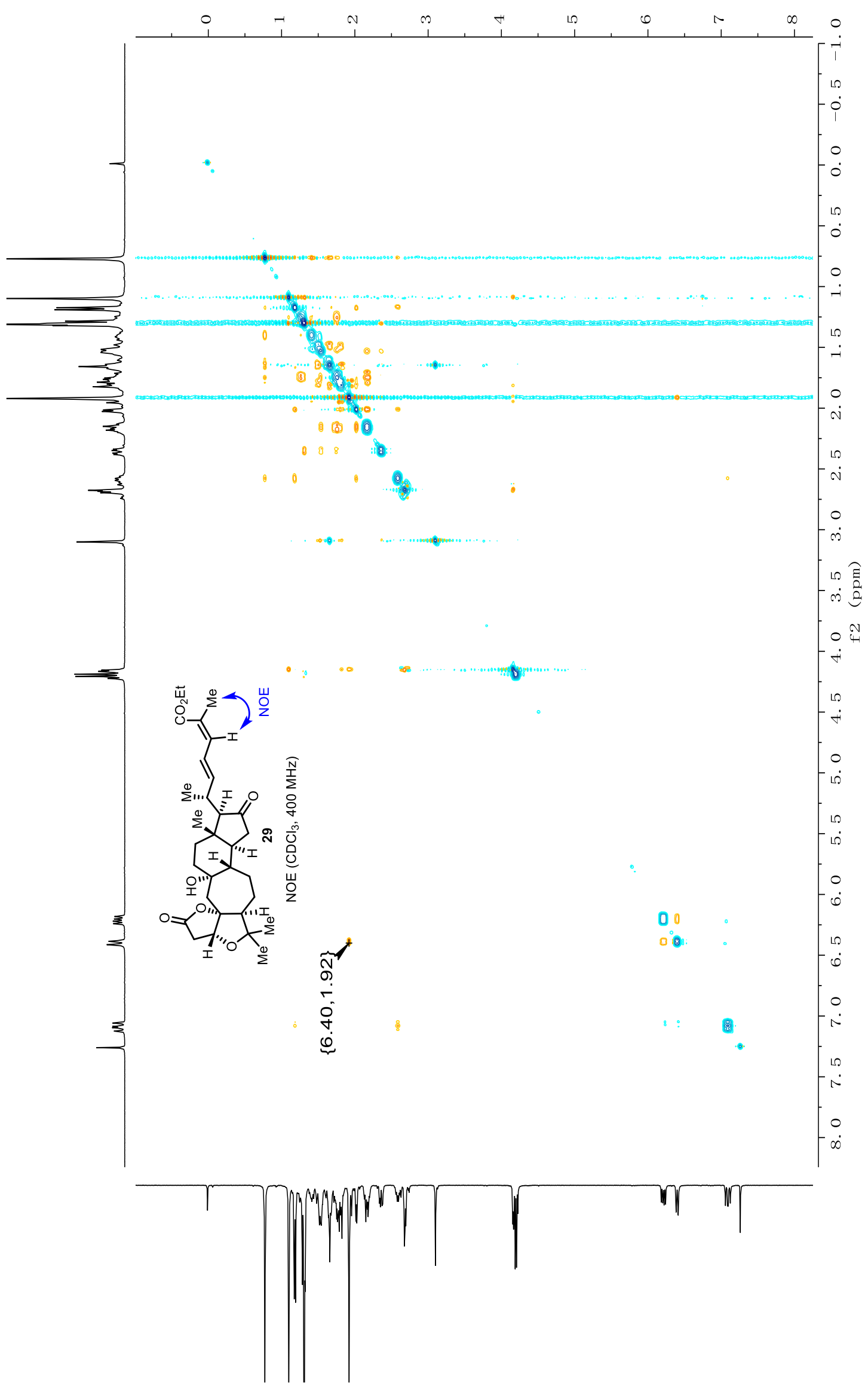



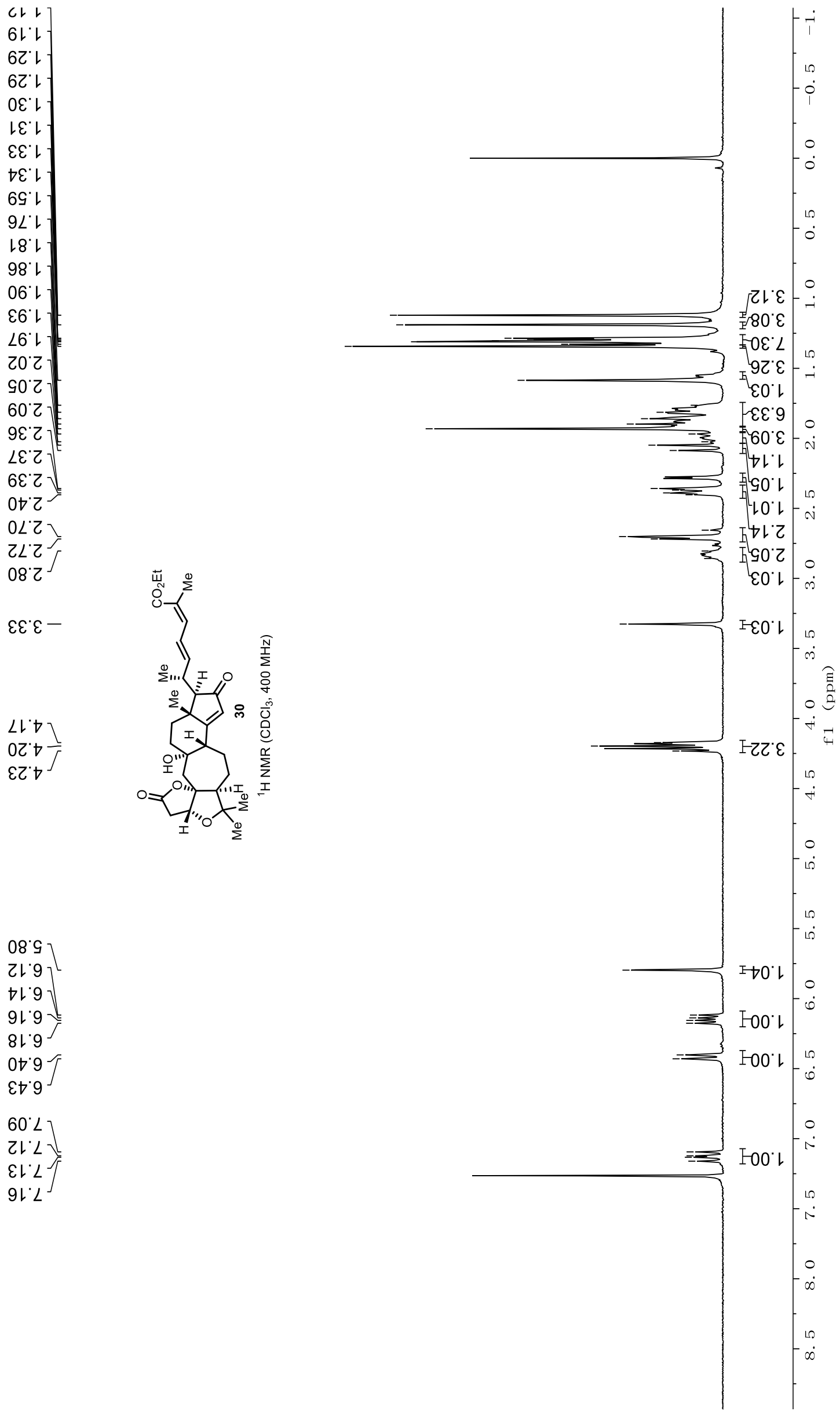


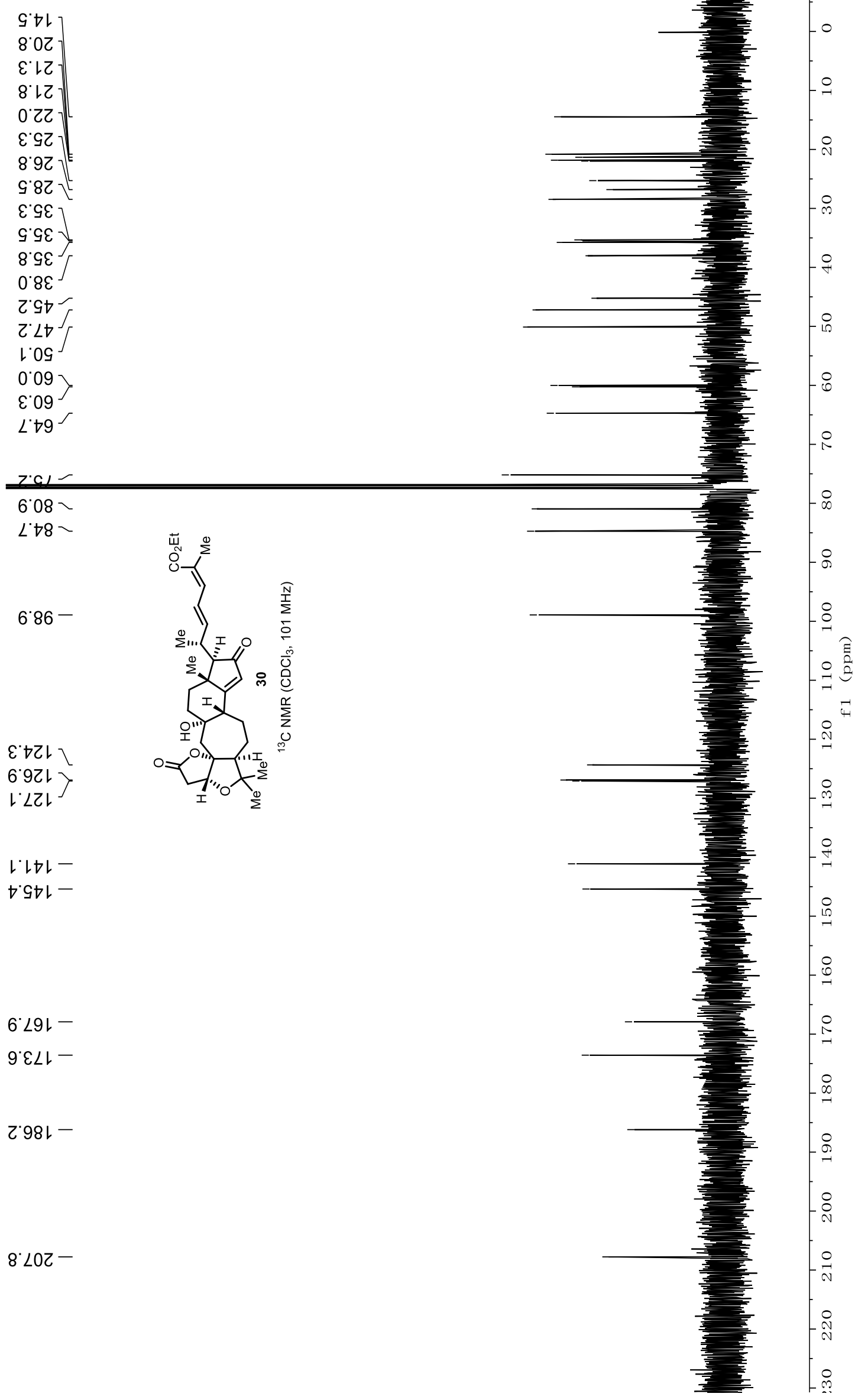



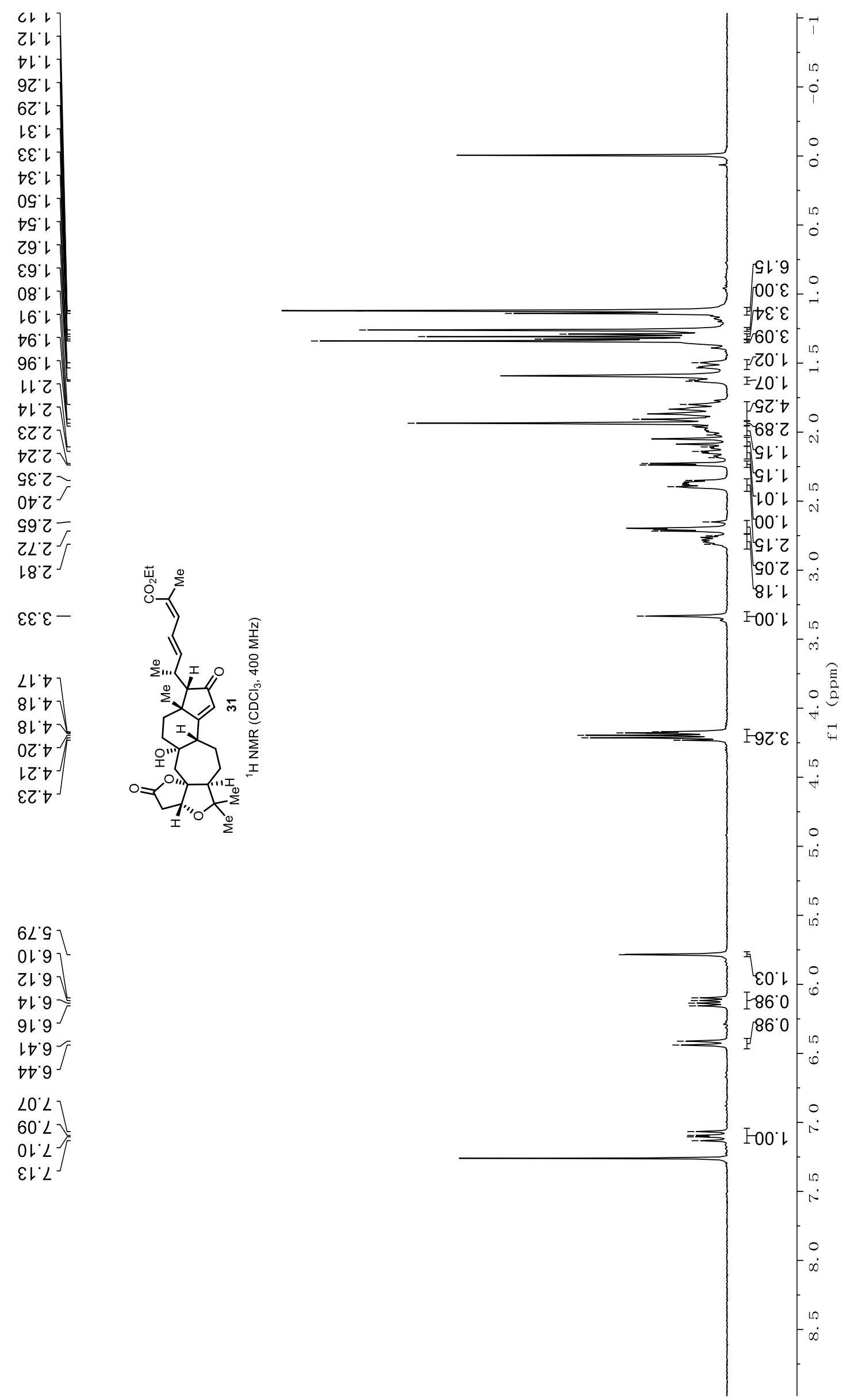


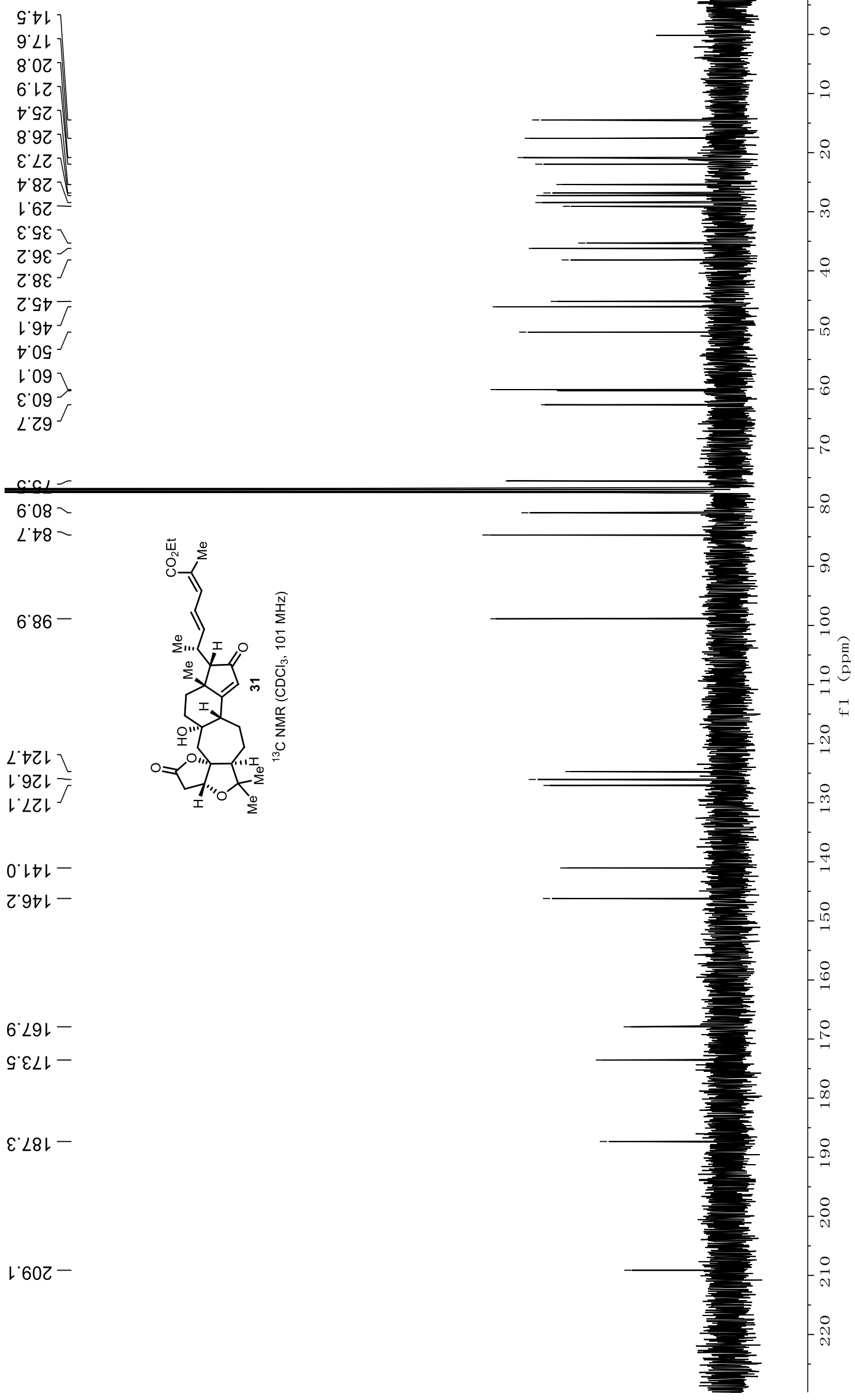




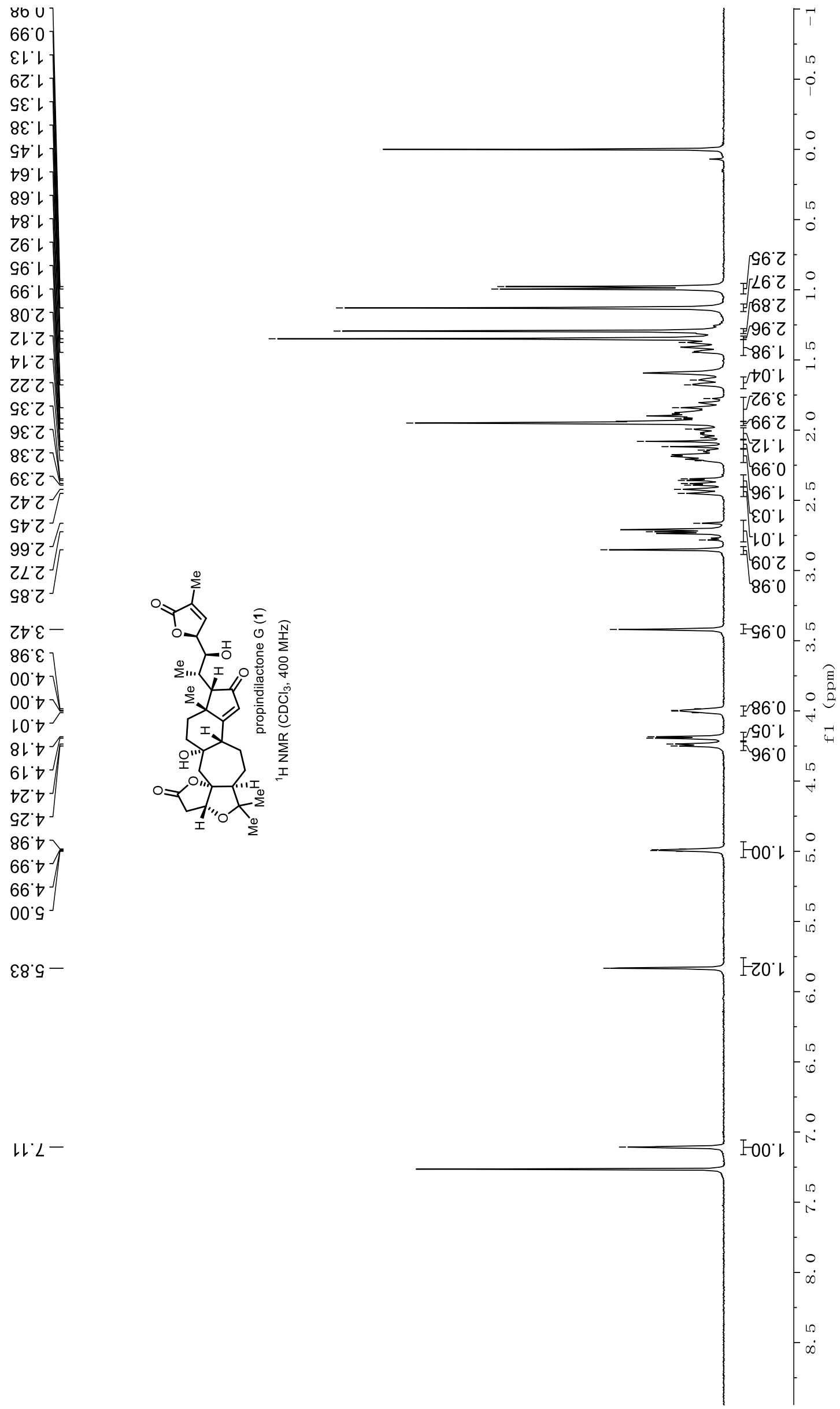




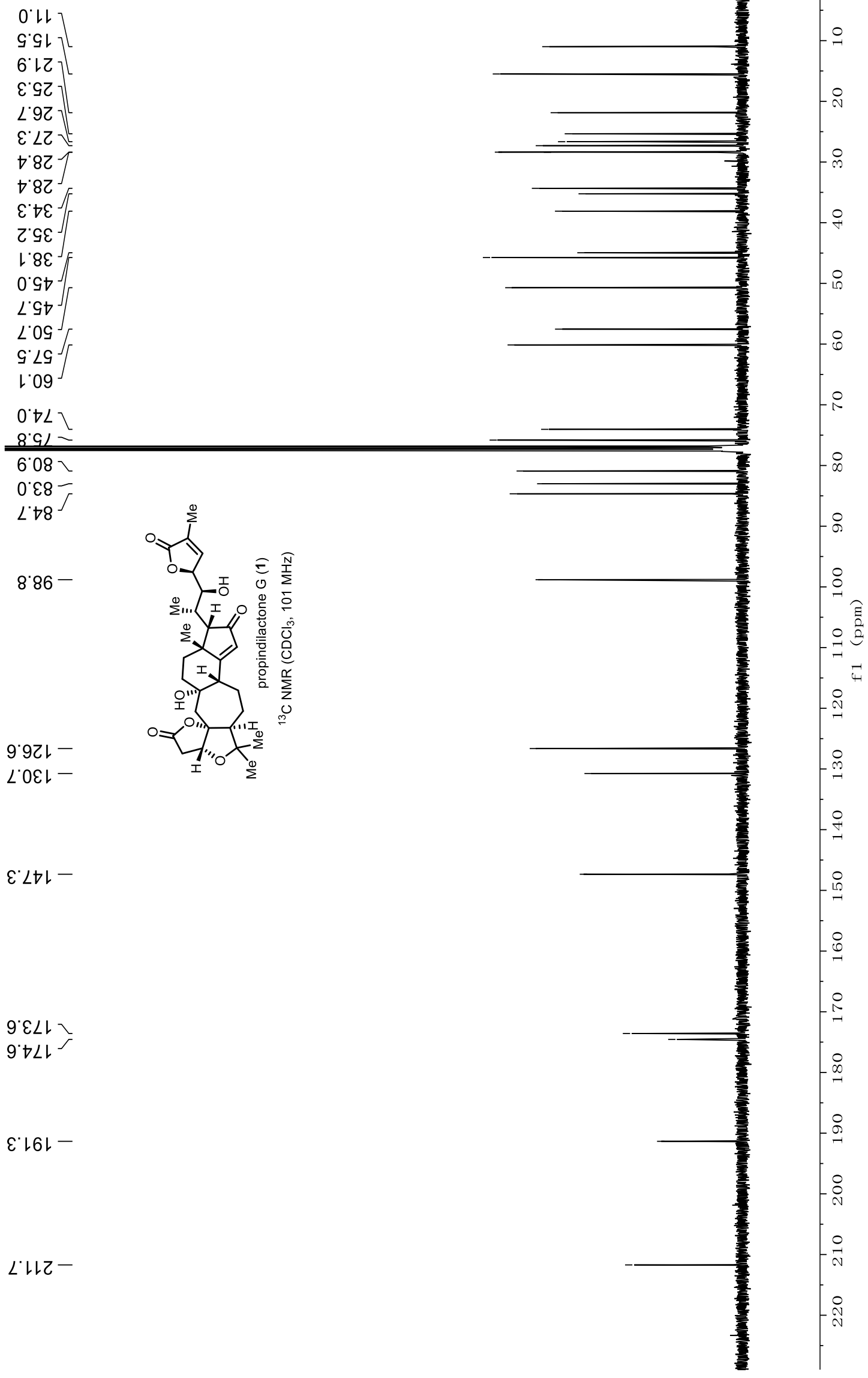




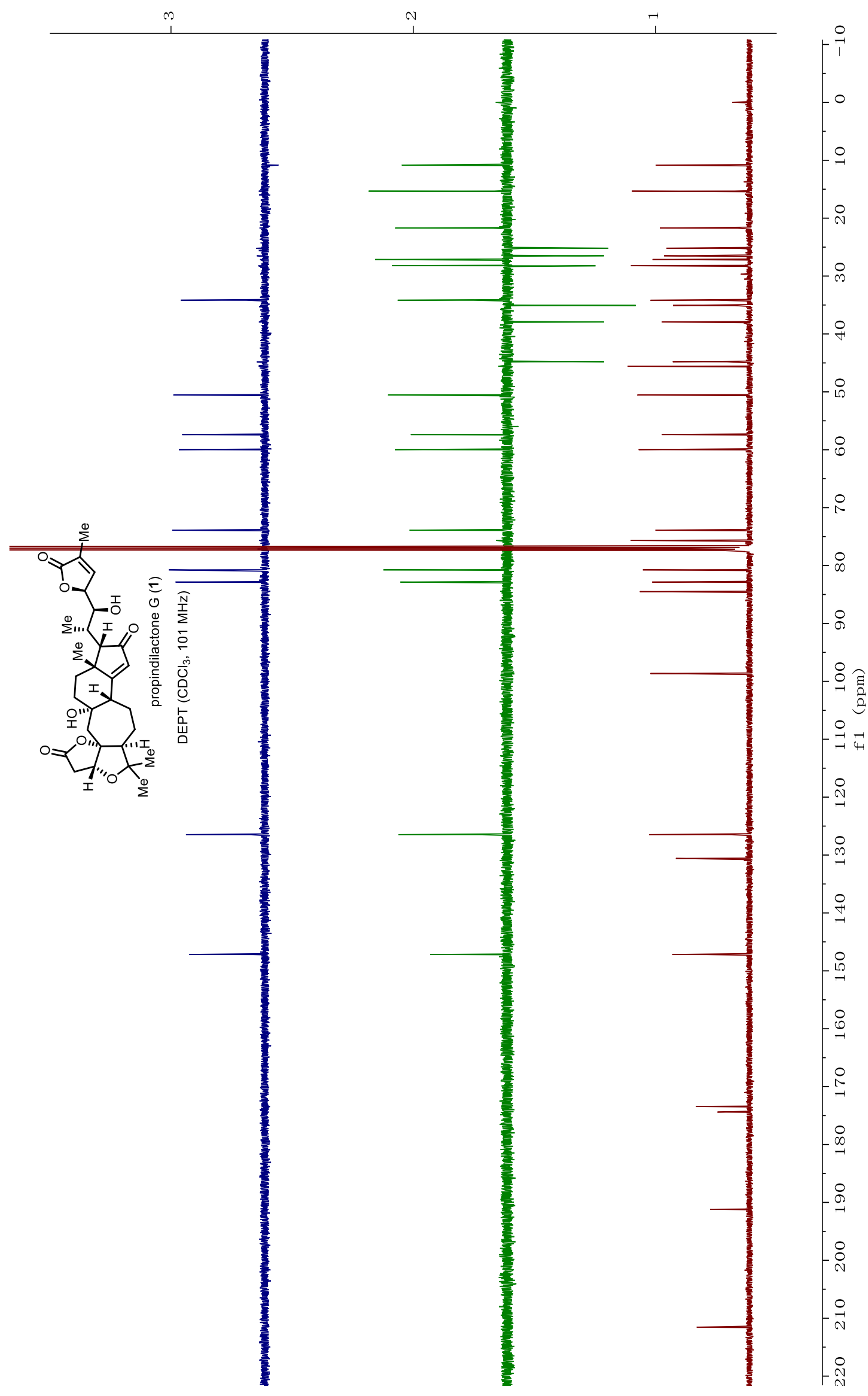


(udd) If

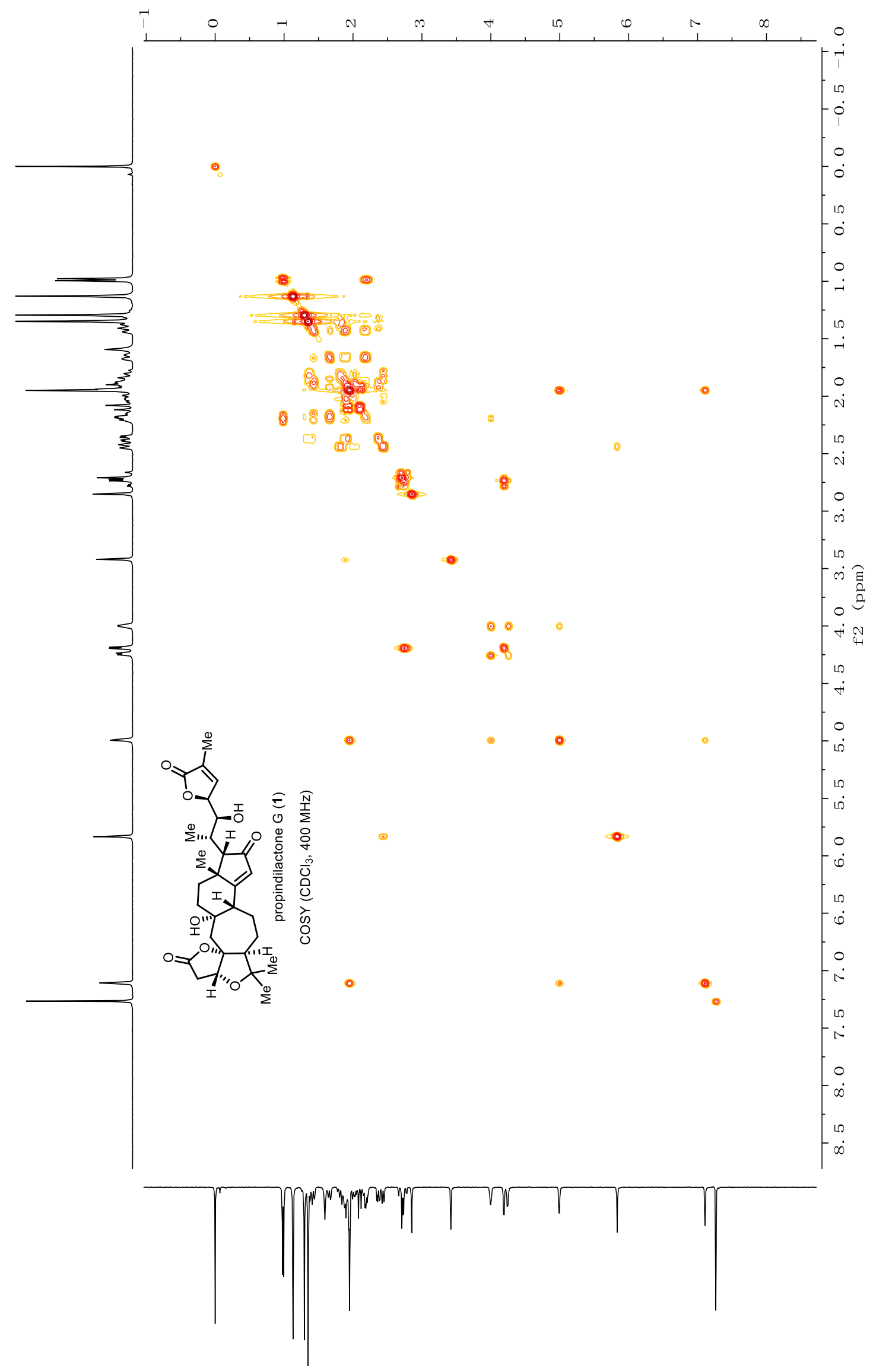


(udd) If

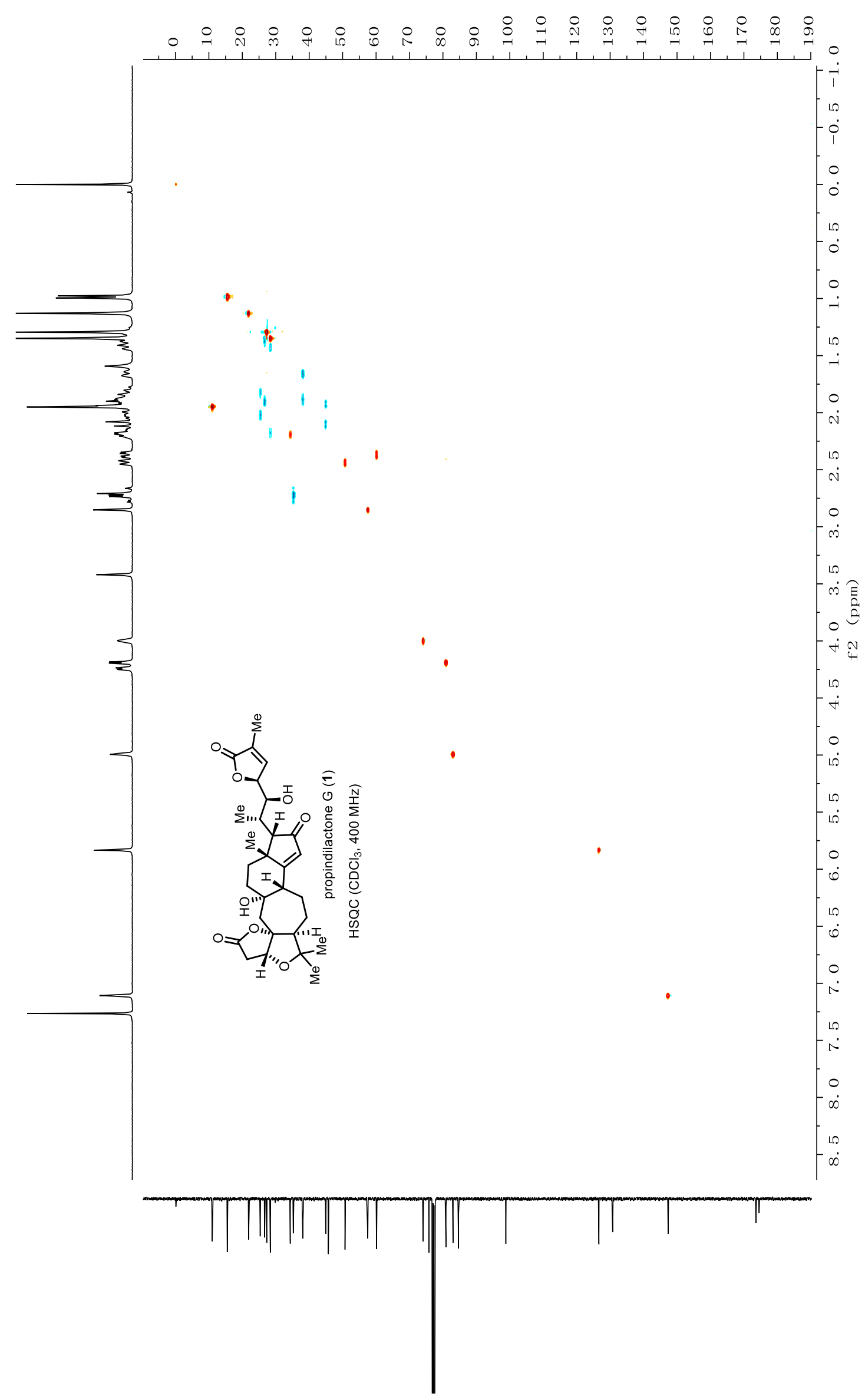


(udd) If

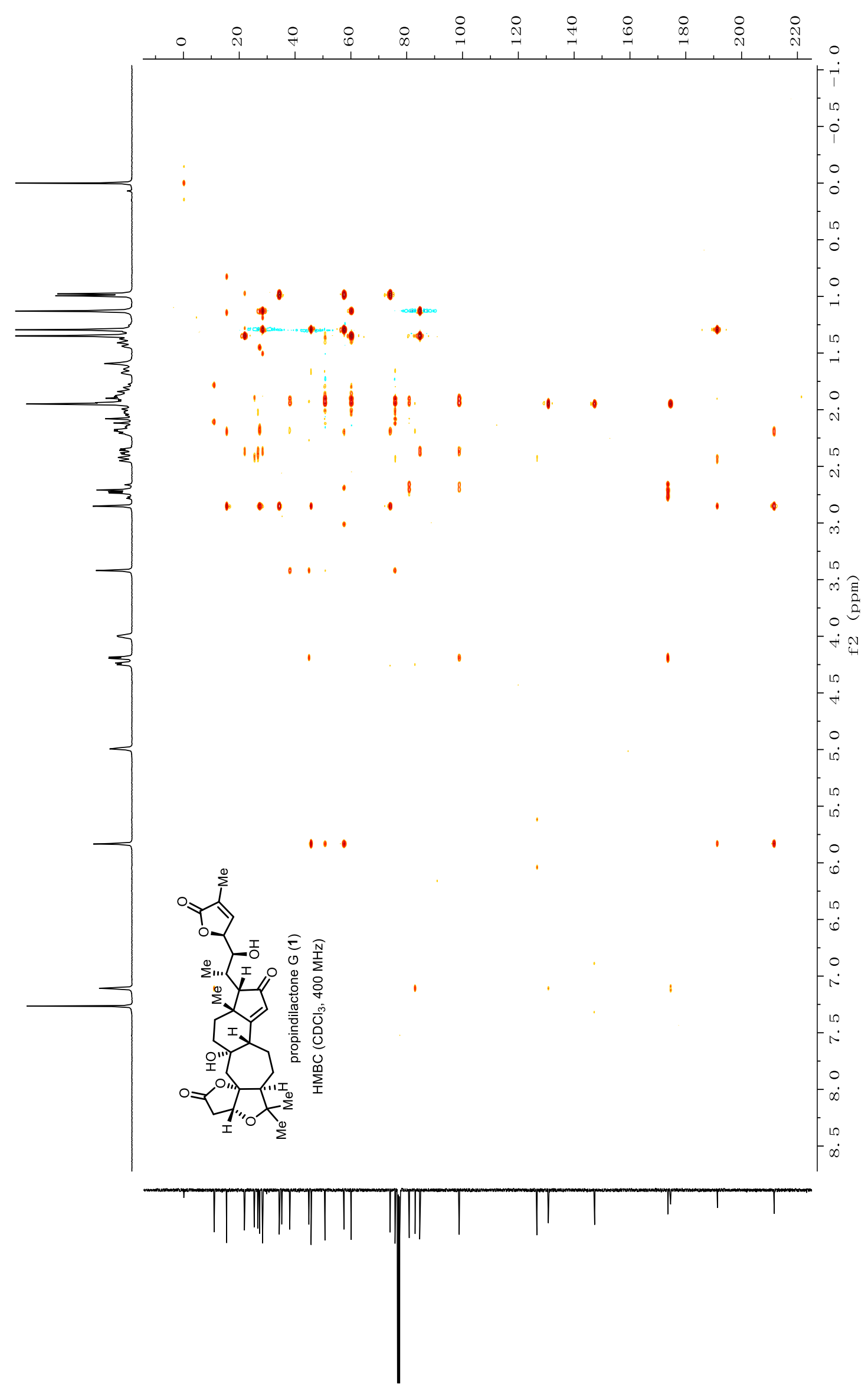


(udd) If

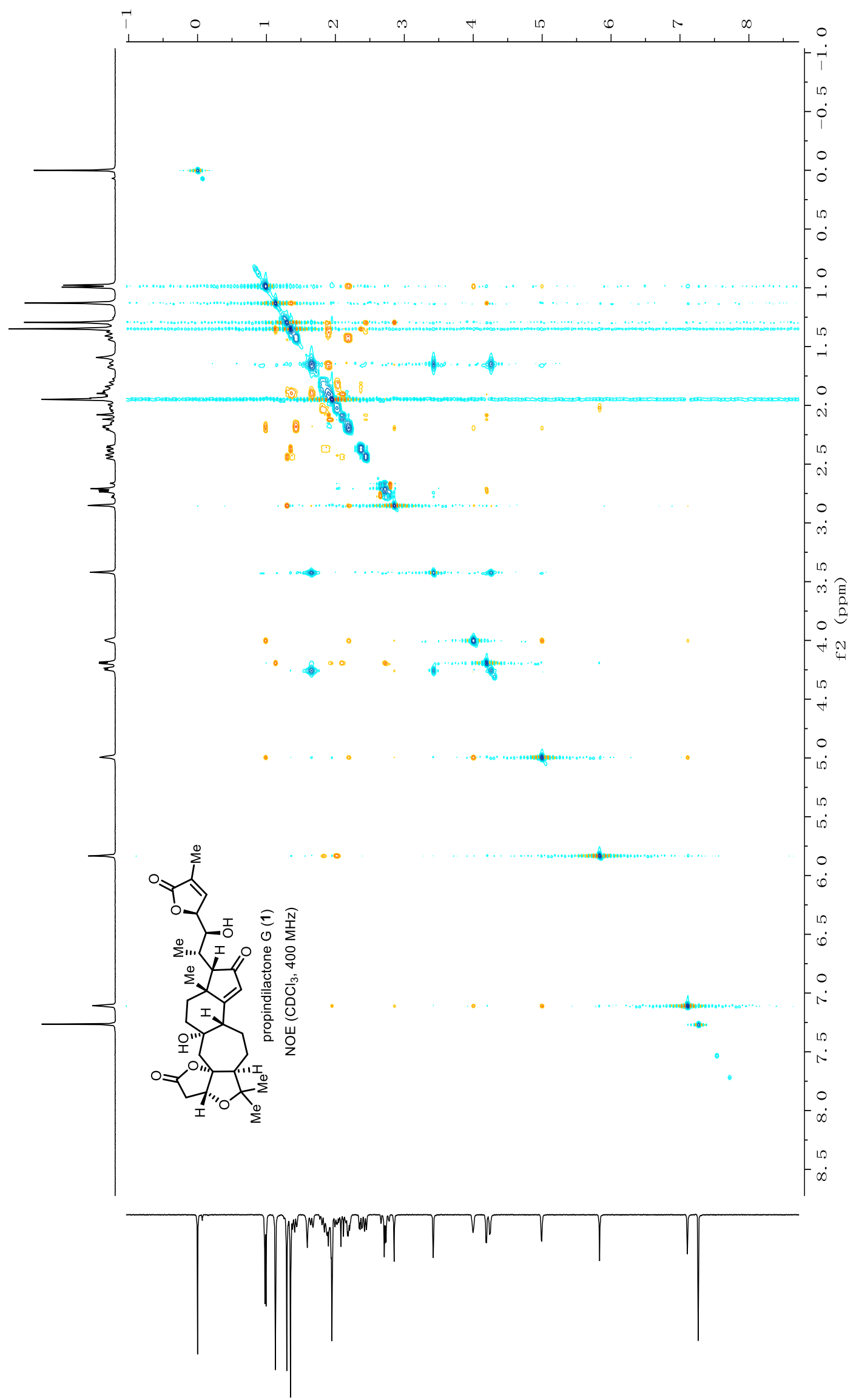




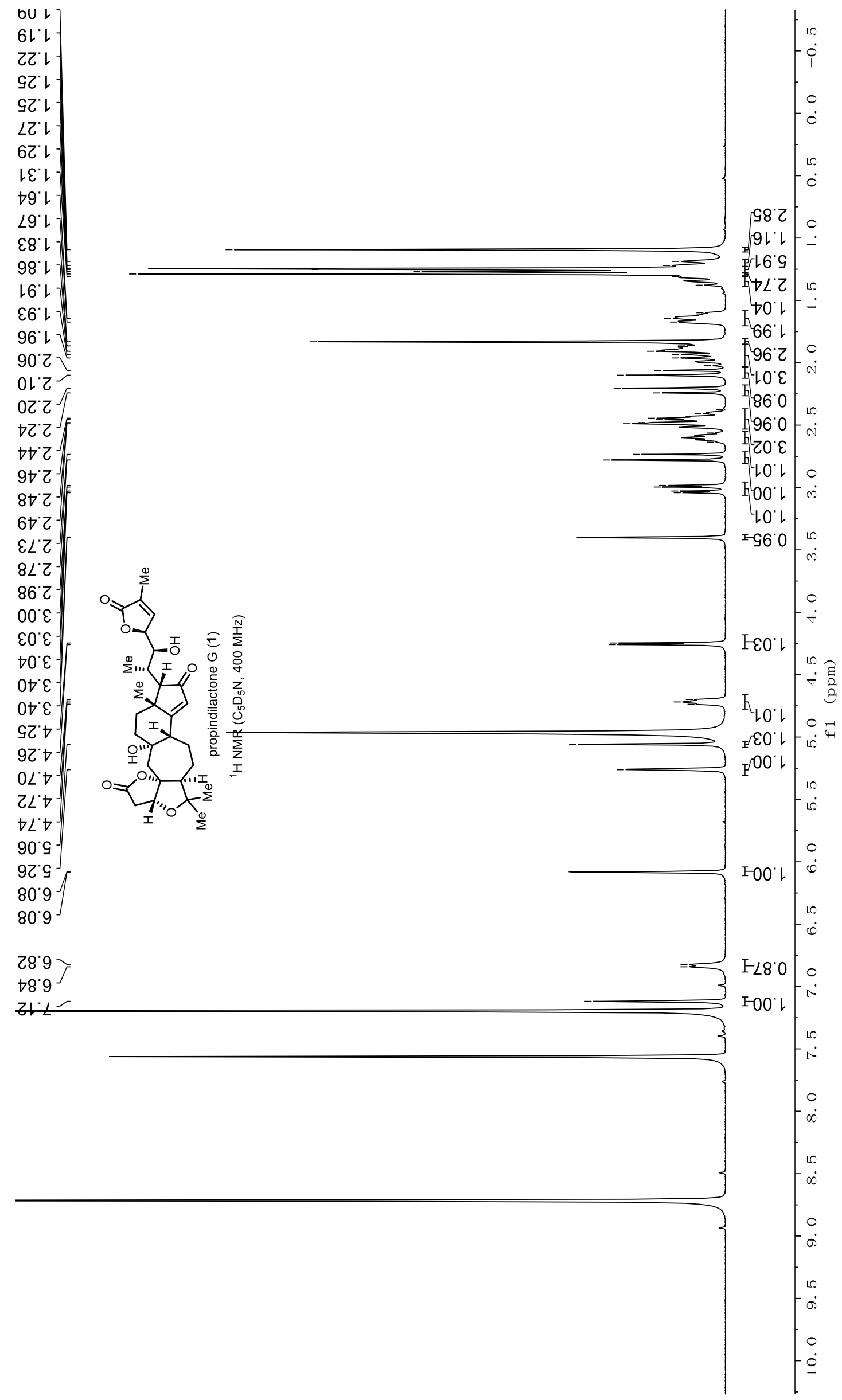



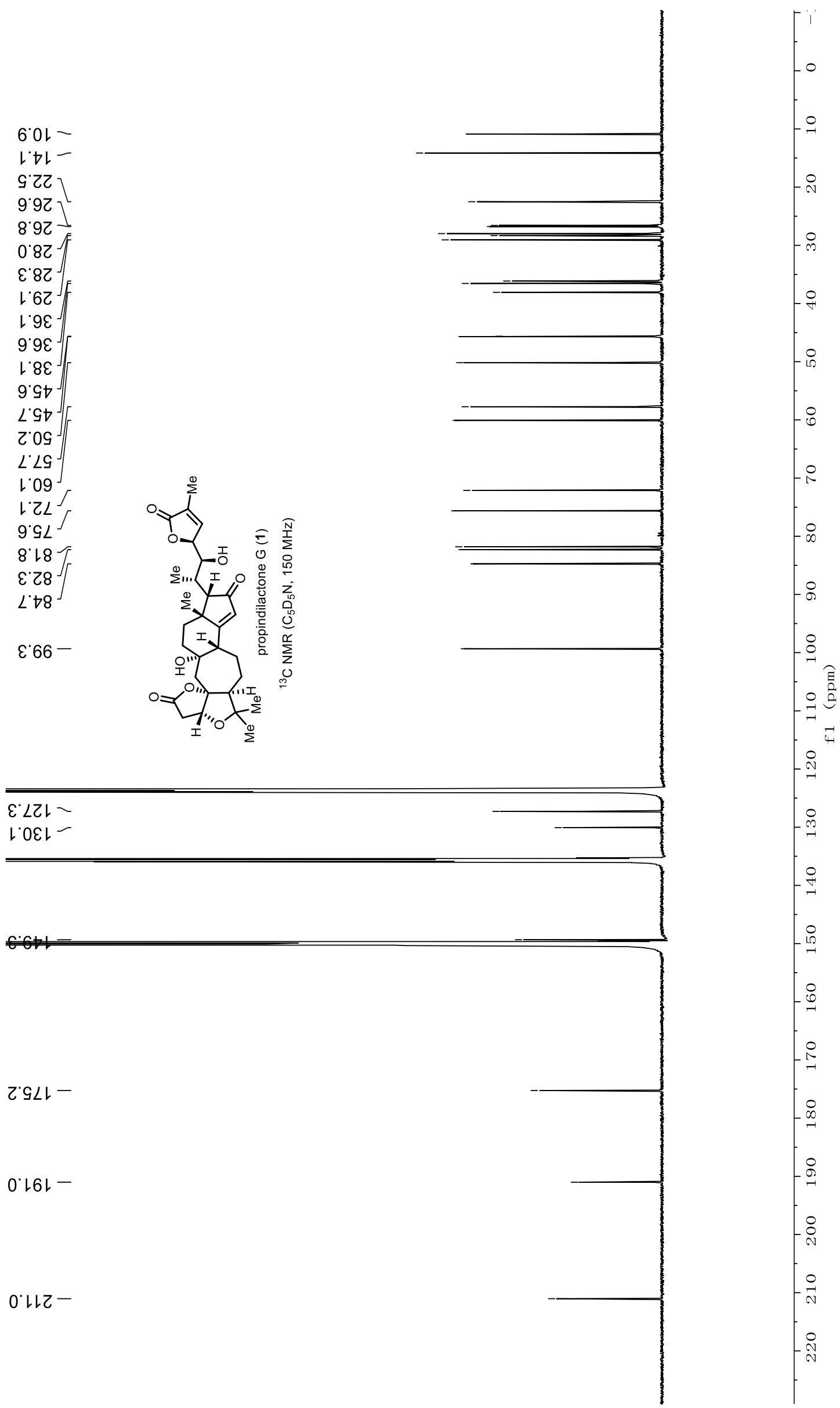


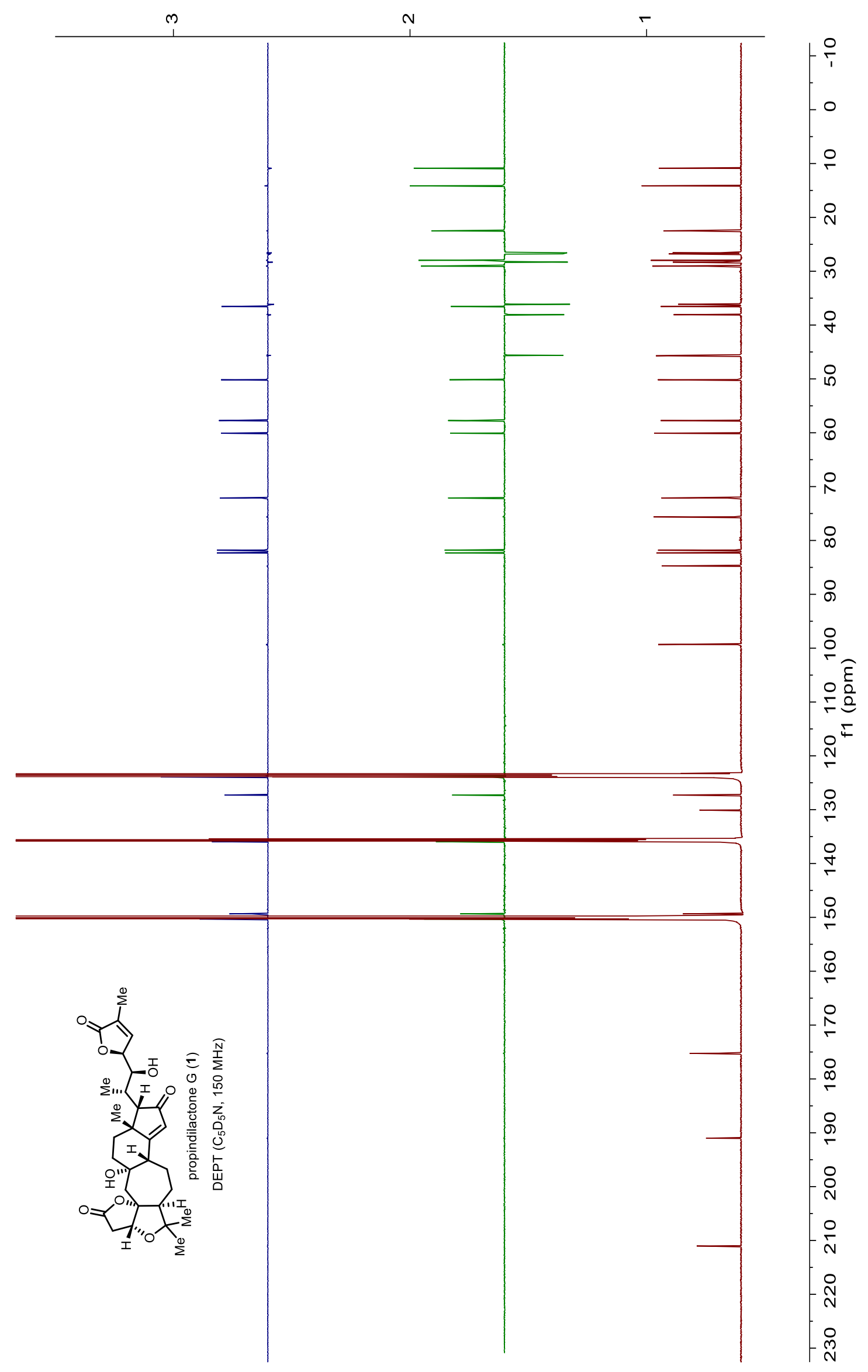


(wdd) If

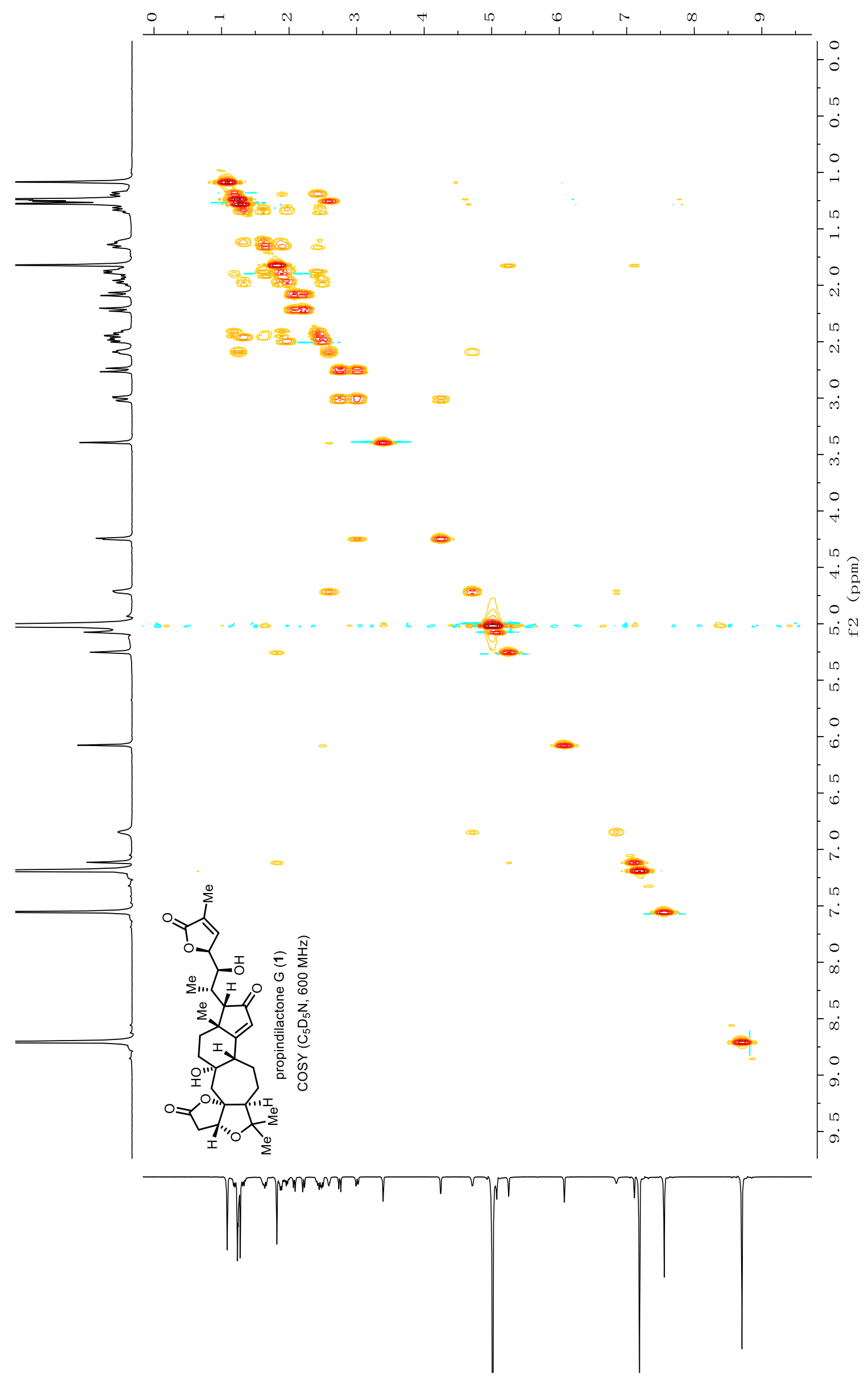


(udd) If

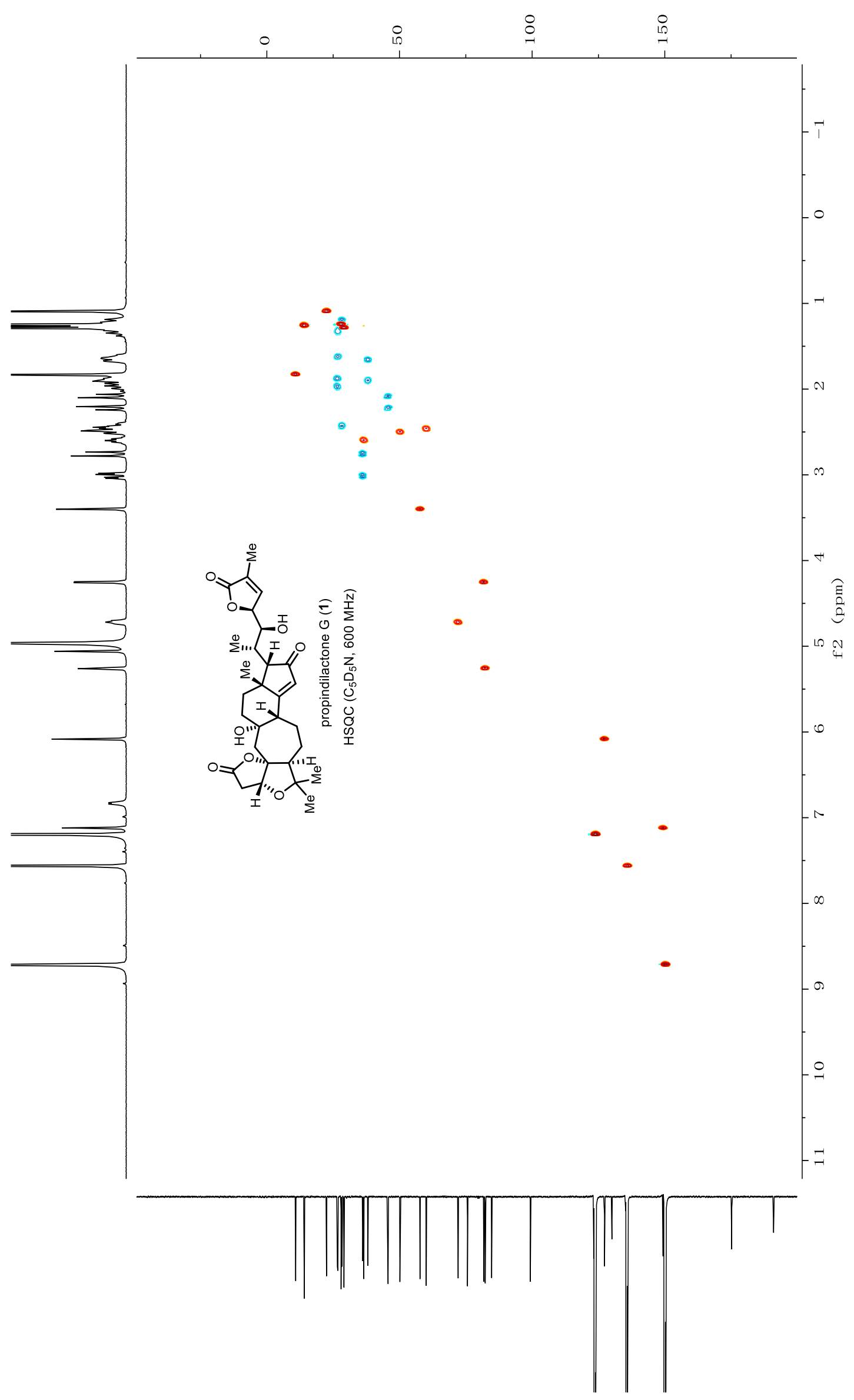


(wdd) IJ

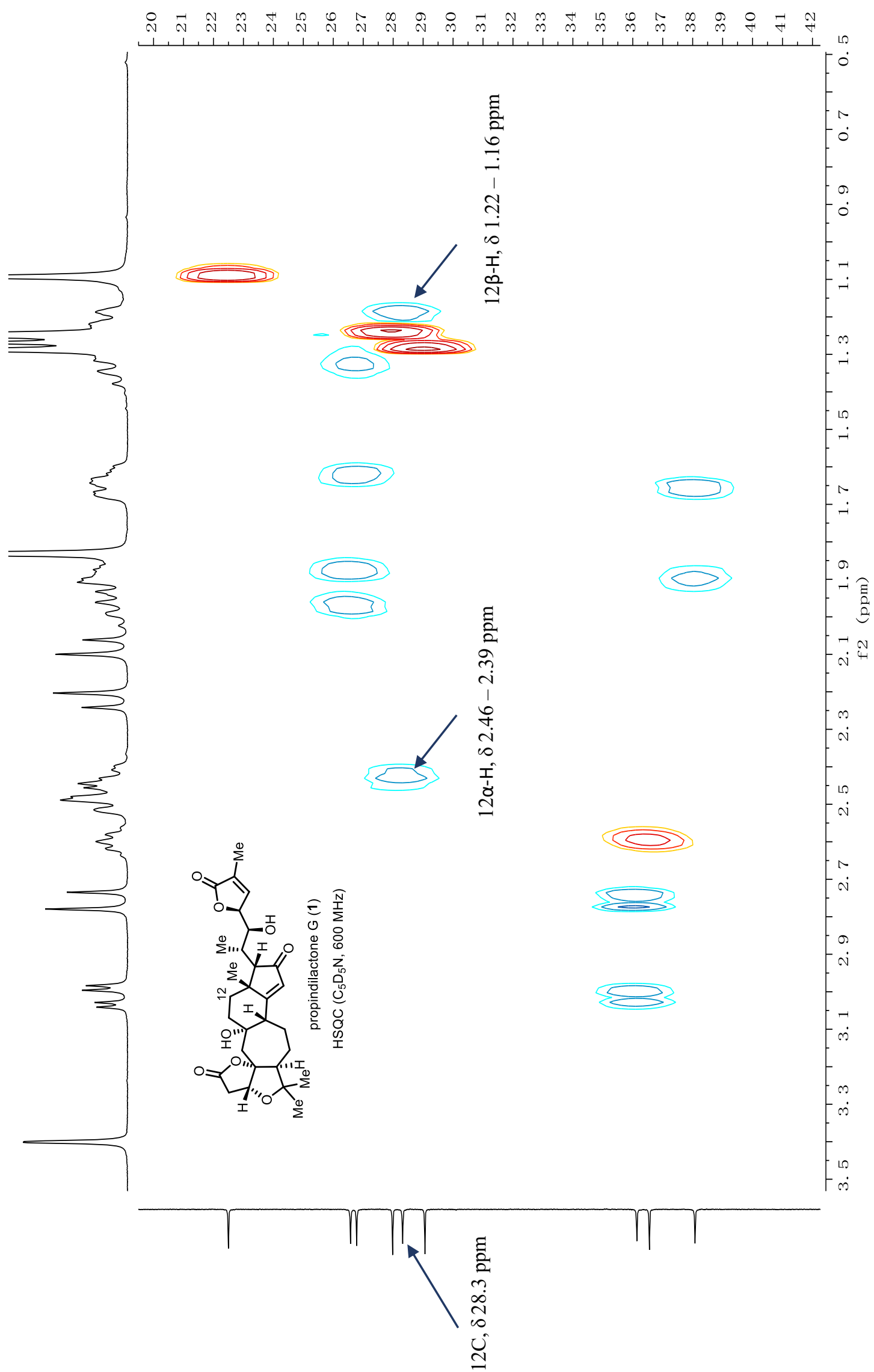


(wdd) IJ

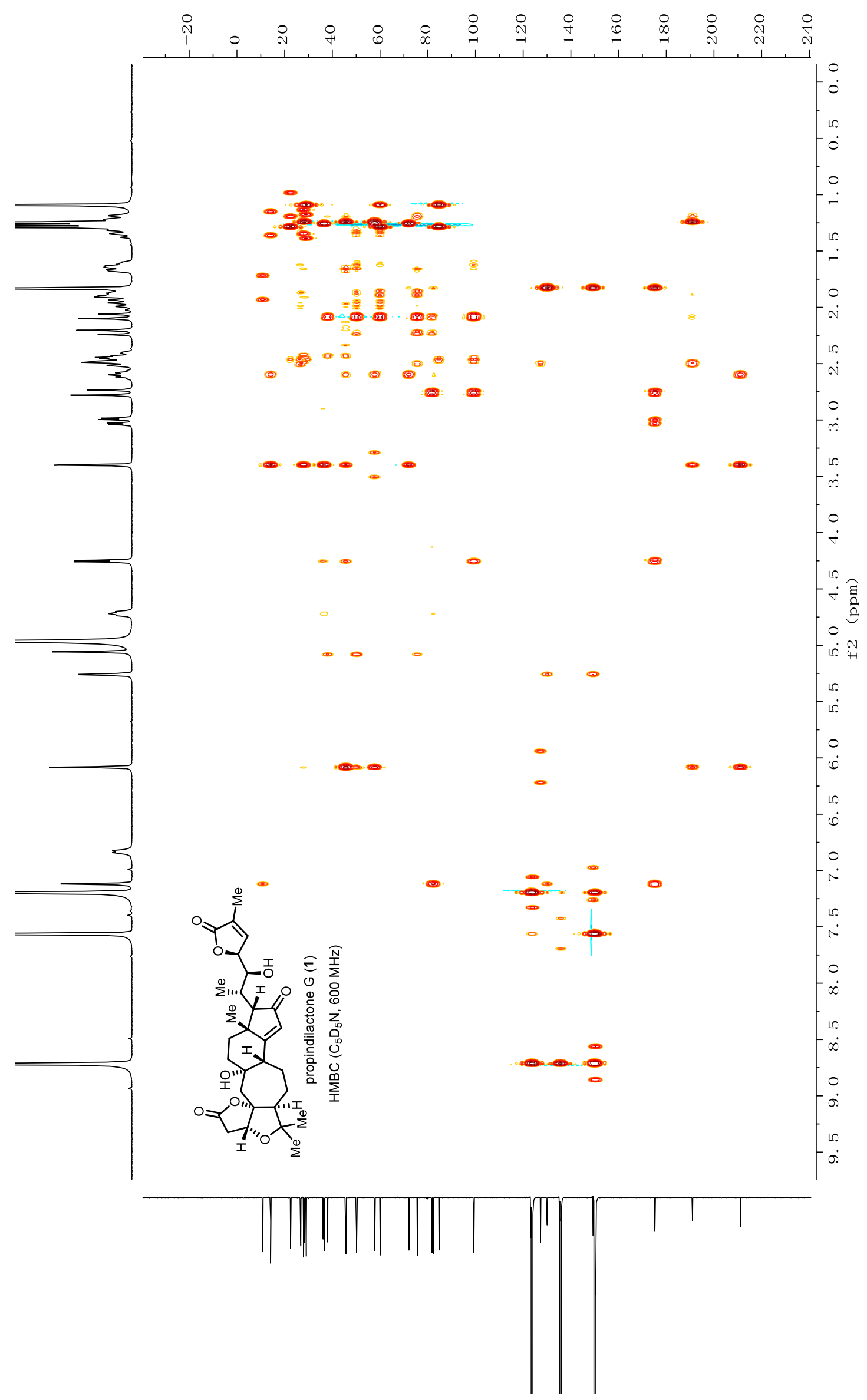


(wdd) IJ

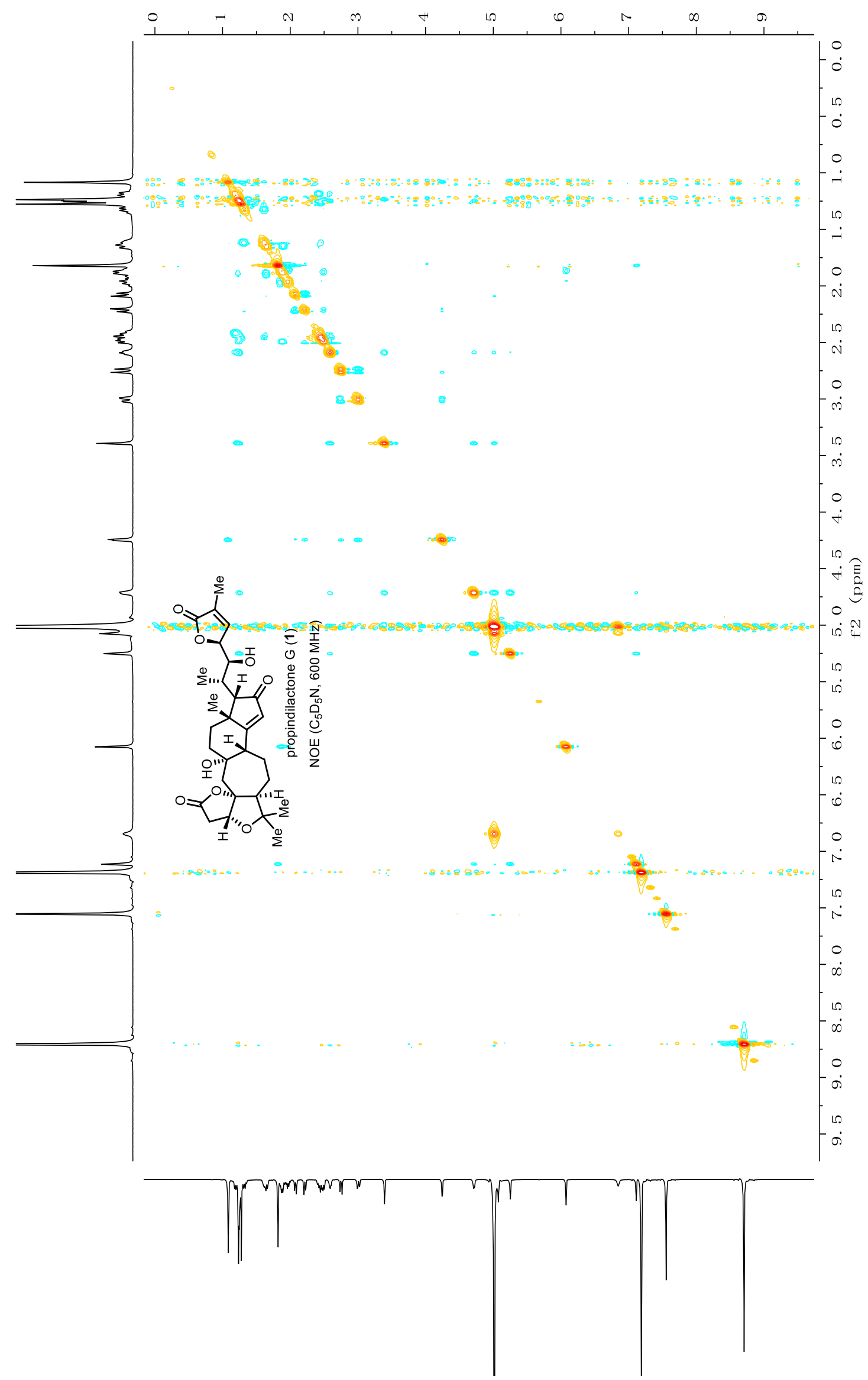




\section{References}

(1) Wang, S.-S.; Shi, Y.; Tian, W.-S. Highly efficient and scalable synthesis of clionamine D. Org. Lett. 2014, 16, 2177.

(2) Lei, C.; Huang, S.-X.; Chen, J.-J.; Yang, L.-B.; Xiao, W.-L.; Chang, Y.; Lu, Y.; Huang, H.; Pu, J.X.; Sun, H.-D. Propindilactones E-J, Schiartane nortriterpenoids from Schisandra propinqua var. propinqua. J. Nat. Prod. 2008, 71, 1228.

(3) You, L.; Liang, X.-T.; Xu, L.-M.; Wang, Y.-F.; Zhang, J.-J.; Su, Q.; Li, Y.-H.; Zhang, B.; Yang, S.L.; Chen, J.-H.; Yang, Z. Asymmetric total synthesis of propindilactone G. J. Am. Chem. Soc. 2015, 137, 10120. 